\title{
IN VITRO GROWTH OF OSTEOBLASTS ON POLY LACTIC-CO-GLYCOLIC ACID SCAFFOLDS CREATED VIA GAS FOAMING
}

\author{
A Thesis \\ presented to \\ the Faculty of California Polytechnic State University, \\ San Luis Obispo
}

\begin{abstract}
In Partial Fulfillment
of the Requirements for the Degree

Master of Biomedical Engineering
\end{abstract}

by

Matthew J. Thomas

August 2018 
(C)2018

Matthew James Thomas

ALL RIGHTS RESERVED 


\section{COMMITTEE MEMBERSHIP}

TITLE: In Vitro Growth of Osteoblasts on Poly Lactic-coGlycolic Acid Scaffolds Created Via Gas Foaming

AUTHOR: $\quad$ Matthew James Thomas

DATE SUBMITTED: August 2018

COMMITTEE MEMBER: Christopher Heylman, Ph.D.

Assistant Professor of Biomedical Engineering

COMMITTEE MEMBER: Kristen Cardinal, Ph. D

Professor of Biomedical Engineering

COMMITTEE MEMBER: $\quad$ Scott Hazelwood, Ph. D

Professor of Biomedical Engineering 


\begin{abstract}
In Vitro Growth of Osteoblasts on Poly Lactic-co-Glycolic Acid Scaffolds Created Via Gas Foaming Matthew James Thomas
\end{abstract}

This study analyzed the feasibility of using gas foaming to create Poly Lactic-co-Glycolic Acid (PLGA) scaffolds for use as a substrate in bone tissue engineering and set out to determine whether the presence of osteoblasts on these scaffolds enhanced their material stiffness. The process of bone formation involves osteoblasts depositing extracellular matrix and calcifying this matrix with calcium phosphate crystals (Hasegawa et al., 2017) and pits between 30-40 $\mu \mathrm{m}$ in diameter on tissue engineering scaffold surfaces have been shown to best promote osteogenic activity in the presence of bone-forming cells (Halai et al., 2014). The scaffolds were determined to contain pits within this $30-40 \mu \mathrm{m}$ range and the ability of osteoblasts to lay down and calcify extracellular matrix on gas foamed PLGA scaffolds was confirmed by the image analysis of inverted optical microscope images of Alizarin Red S-stained scaffold cryosections. The presence of osteogenic activity combined with the desired scaffold porosity led us to conclude gas foaming PLGA scaffolds is a feasible method of scaffold fabrication for bone tissue engineering and allowed us to solidify the optimized gas foaming apparatus as an instrument to be used in further bone tissue engineering experiments at California Polytechnic State University, San Luis Obispo. However, this study failed to determine whether the presence of osteoblasts improved the material stiffness of the PLGA scaffolds due to a lack of statistical significance in compression testing results.

Keywords: Osteoblasts, Gas Foaming, PLGA 


\section{ACKNOWLEDGMENTS}

I would personally like to thank the California Polytechnic State University of San Luis Obispo's College of Engineering Return Indirect Costs program (R-IDC) for funding this project.

I would like to thank Dr. Kristen Cardinal, Dr. Scott Hazelwood, and Dr. Christopher Heylman for serving on my thesis committee.

I would like to further thank Dr. Christopher Heylman for helping guide me towards the completion of this project. He dedicated much of his time to ensure this project was completed in a timely and fully-realized manner and its completion would not have been possible without him.

I would like to thank Leah Torres for designing the compression platens used in Solidworks, for helping fabricate the PLGA scaffolds tested, and for helping freeze the scaffolds in optimum cutting temperature gel.

I would like to thank Dr. Richard Savage for teaching me how to use the JEOL JSM-6390 Scanning Electron Microscope.

I would like to thank Dr. Christy Strand for teaching me how to use the ThermoScientific Cryotome FSE cryostat.

I would like to thank Lily Wong for teaching me how to properly freeze material in optimum cutting temperature gel.

I would like to thank Borna Sobati for helping fabricate the PLGA scaffolds tested.

Lastly, I would like to thank Kendyl Cohn for various advice along the way. 


\section{LIST OF TABLES}

Table

4.1 Line of best fit constants from Fig 4.1 and the coefficient of variation $(\mathrm{CoV})$ for each constant between gas foaming releases

4.2 Line of best fit constants from Fig 4.2 and the coefficient of variation $(\mathrm{CoV})$ for each constant between gas foaming releases

4.3 Calculated percent difference between the coefficients of variations $(\mathrm{CoV})$, a measure of difference in variability, in the lines of best fit constants for the two gas foaming methods 44

4.4 Mean values of pixels determined to be pores in ImageJ and the resulting F-stat and P-value from running an ANOVA in JMP 12 on the number of measured pore pixels in the SEM images of scaffolds made via the different gas foaming methods

4.5 Mean values of pixels determined to be Alizarin Red S-stained material in ImageJ and the resulting F-stat and P-value from running an ANOVA in JMP 12

4.6 P-values from all pairs Tukey's HSD output comparing individual treatment groups with each other for the amount of Alizarin Red S-stained pixels in each inverted optical microscope image..........57

4.7 Table of Young's moduli of each scaffold compressed in each treatment group in MPa

4.8 The interquartile range and first quantile of the Young's moduli values for the compression tests run on the osteoblast-containing scaffolds.

4.9 Individual student's T-test Young's moduli between each pair of scaffold types including the 128.73 value $(\alpha=0.05)$

4.10 Individual student's T-test Young's moduli between each pair of scaffold types excluding the 128.73 value $(\alpha=0.05)$

A.1 Summarized line-of-best-fit values from each PID run in Figures A.2 - A.8 .75 
A.2 Average line-of-best-fit values from PID runs in Figures A. 2 - A.8 and their percent difference from the ideal values.

D.1 Osteoblast-Containing Scaffolds Specimen 1 Raw Compression Data ..................................................... 88

D.2 Osteoblast-Containing Scaffolds Specimen 2 Raw Compression Data ................................................. 91

D.3 Osteoblast-Containing Scaffolds Specimen 3 Raw Compression Data ................................................. 94

D.4 Osteoblast-Containing Scaffolds Specimen 4 Raw Compression Data .................................................... 97

D.5 ODM-Soaked Scaffolds Without Osteoblasts Specimen 1 Raw Compression Data.............................. 100

D.6 ODM-Soaked Scaffolds Without Osteoblasts Specimen 2 Raw Compression Data............................ 103

D.7 ODM-Soaked Scaffolds Without Osteoblasts Specimen 3 Raw Compression Data.............................. 106

D.8 ODM-Soaked Scaffolds Without Osteoblasts Specimen 4 Raw Compression Data............................ 109

D.9 PBS-Soaked Scaffolds Specimen 1 Raw Compression Data..................................................................... 112

D.10 PBS-Soaked Scaffolds Specimen 2 Raw Compression Data ............................................................. 115

D.11 PBS-Soaked Scaffolds Specimen 3 Raw Compression Data ................................................................ 118

D.12 PBS-Soaked Scaffolds Specimen 4 Raw Compression Data .............................................................. 121

D.13 Dry Scaffolds Specimen 1 Raw Compression Data........................................................................... 124

D.14 Dry Scaffolds Specimen 2 Raw Compression Data.................................................................................. 127

D.15 Dry Scaffolds Specimen 3 Raw Compression Data........................................................................... 130

D.16 Dry Scaffolds Specimen 4 Raw Compression Data............................................................................. 133 


\section{LIST OF FIGURES}

Figure $\quad$ Page

2.1 Scanning electron microscope (SEM) images of tissue scaffolds created via a) electrospinning

(Bhardwaj \& Kundu, 2010), b) solvent casting and particulate leaching (Liao et al., 2002), and

c) gas-foaming and particulate leaching (Harris et al., 1998) compared to d) an SEM image of

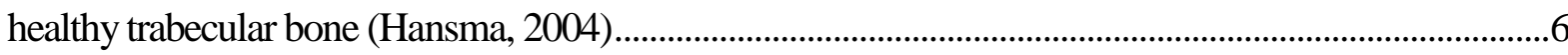

2.2 Visual representation of the molecular organization of amorphous vs. semicrystalline polymers

(MacVarish, 2017)

2.3 Time until polymer is to half of its density after degrading in water based on ratio of PLA to

PGA present in PLGA (Felton, 2011)

2.4 An unsuccessful PLGA scaffold creation attempt where pressure was released too rapidly from

the gas foaming chamber

2.5 Basic block diagram of a process controlled by a PID (Knospe, 2006) .

2.6 Light micrograph image of mesenchymal stem cells clustering to form a nidus (Brighton \& Hunt, 1997)

2.7 Transmission Electron Microscope images of a matrix vesicle secreted from an osteoblast during osteogenesis. a) shows the beginning of crystallization on the inner plasma membrane, b) shows the growth of calcium-phosphate crystals within the vesicle, and c) shows the crystals tearing through the plasma membrane (Hasegawa et al., 2017) ............................................................... 13

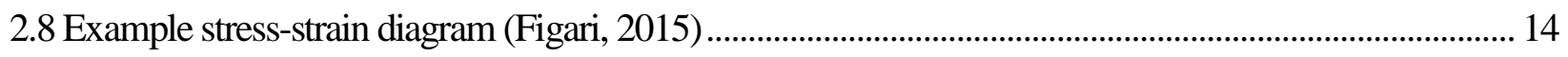

2.9 Alizarin Red S staining of calcium deposits from pre-osteoblast MC3T3-E1s (Li et al., 2016).............. 15

3.1 a) Eisco Labs porcelain mortar and pestle b) American Educational screen sieves set ............................. 17

3.2 a) $5 \mathrm{~mm}$ Type KB Precision die b) Die under $2 \mathrm{kN}$ press c) Pressed pellets of mixed PLGA and salt 
3.3 Schematic of DAQ setup. DAQ reads pressure in terms of AC current and sends a pressure reading to the NI LabView program. The NI LabView program sends a signal for a specified $\mathrm{AC}$ voltage to the $\mathrm{DAQ}$ which sends that $\mathrm{AC}$ voltage to the positioner on the release valve.

3.4 a) Removable shelf for scaffolds in gas foaming chamber, b) Demonstration of shelf being removed from the gas foaming chamber, c) Gas foaming chamber and Valworx automated release valve and positioner

3.6 a) Denton Vacuum Desk IV gold-sputtering scaffold top surfaces, b) JEOL JSM-6390 SEM, c) loading a gold-sputtered scaffold into the SEM.

3.7 Threshold Color settings in Fiji ImageJ for detecting pores in the surface of PLGA scaffolds

$3.8 \mathrm{a} \& d$ ) Original SEM images of scaffolds, b\&e) Filtered SEM images of scaffolds, c\&f) Filtered regions selected for measurements.

3.9 Osteoblast plating procedure flowchart

3.10 Picture of osteoblasts at $\sim 80 \%$ confluency taken under an optical microscope 27

3.11 Osteoblast expansion flowchart ………………………………………………………………….. 28

3.12 Three cryovials of frozen human adult osteoblasts................................................................................. 31

3.13 48-well plate setup for scaffold soak and osteoblast growth with the respective media in each well listed on the left and the dedicated experiment for each well listed above

3.14 Instron 3342 single-column tensile/compression testing machine with top and bottom compression platens attached.

3.15 a) Scaffolds frozen in OCT gel in molds, b) Frozen OCT gel block in cryostat on die, c) Five cryosections from a dPBS-soaked scaffold on a Vectabond-treated slide 38 
3.16 a) Cryosections being pulled out of Alizarin Red S solution, b) Slides after Alizarin Red S staining 40

3.17 Threshold Color settings in Fiji ImageJ for detecting Alizarin Red S-stained material. 40

$3.18 \mathrm{a} \& \mathrm{~d})$ Original inverted optical microscope images of Alizarin Red S-stained cryosections, b\&e) Filtered images of Alizarin Red S-stained cryosections, c\&f) Filtered regions selected for measurements

4.1 Pressure vs. time graphs of six consecutive gas releases utilizing the PID to control carbon dioxide gas release

4.2 Pressure vs. Time graphs of six consecutive gas releases where a constant $2 \mathrm{~V}$ was sent to the positioner.

4.3 SEM images taken at 30x magnification of scaffolds made by ( $a \& b)$ using a PID (c\&d) sending a constant $2 \mathrm{~V}$ to the positioner.

4.4 SEM images taken at 100x magnification for scaffolds made utilizing the PID. Images (a-c) are of the scaffold shown in Figure 4.3(a). Images (d-f) are of the scaffold shown in Figure 4.3(b).

4.5 SEM images taken at 100x magnification for scaffolds made by sending a constant $2 \mathrm{~V}$ to the positioner. Images (a-c) are of the scaffold shown in Figure 4.3(c). Images (d-f) are of the scaffold shown in Figure 4.3(d).

4.6 Boxplot created in JMP 12 comparing the number of pixels in an SEM image that are considered to be surface pores in the PLGA scaffolds created via the two different gas foaming methods

4.7. Images taken with an inverted optical microscope at $4 \mathrm{x}$ magnification of scaffold cryosections stained in Alizarin Red S. There are 2 dPBS-soaked scaffolds labeled as dPBS[scaffold \#]. There are 3ODM-soaked scaffolds labeled as ODM[scaffold \#]. There are 3ODM-soaked 
scaffolds that had osteoblasts deposited on them labeled as $\mathrm{Ob}$ [scaffold \#]. Components stained red contain calcium

4.8 Boxplot created in JMP 12 comparing the distribution of amount of Alizarin Red S-stained pixels in each inverted optical microscope image for the three treatment groups. 56

4.9 The compressive load $(\mathrm{N})$ over extension $(\mathrm{mm})$ of each scaffold in each treatment condition. The orange line represents the first scaffold of a treatment group test, the red is the second, the green is the third, and the light blue is the fourth. The load starts increasing at roughly the same point in extension for each sample in a treatment group, but is shown shifted for visibility.

A.1 NI LabView program of PID controller for controlling actuated release valve on gas foaming apparatus

A.2 Three repetitions of gas release over 30 s with a PID setting of $\mathrm{P}=0.056, \mathrm{I}=20000, \mathrm{D}=0.44$

(Chosen PID settings)

A.3 Three repetitions of gas release over 30 s with a $\mathrm{PID}$ setting of $\mathrm{P}=0.055, \mathrm{I}=20000, \mathrm{D}=0.44(-1 \%$

P)

A.4 Three repetitions of gas release over 30 s with a PID setting of $\mathrm{P}=0.057, \mathrm{I}=20000, \mathrm{D}=0.44(+1 \%$

P)

A.5 Three repetitions of gas release over 30 s with a $\mathrm{PID}$ setting of $\mathrm{P}=0.056, \mathrm{I}=19800, \mathrm{D}=0.44(-1 \%$

I).

A.6 Three repetitions of gas release over 30s with a PID setting of $\mathrm{P}=0.056, \mathrm{I}=20200, \mathrm{D}=0.44(+1 \%$

I).

A.7 Three repetitions of gas release over 30s with a $\mathrm{PID}$ setting of $\mathrm{P}=0.056, \mathrm{I}=20000, \mathrm{D}=0.40(-1 \%$

D) 
A.8 Three repetitions of gas release over 30s with a PID setting of $\mathrm{P}=0.056, \mathrm{I}=20000, \mathrm{D}=0.48$

$(+1 \% \mathrm{D})$

B.1 Gas foaming pressure release preformed on 05/10/2018

B.2 Gas foaming pressure release preformed on 05/11/2018 77

B.3 Gas foaming pressure release preformed on 05/12/2018 .78

B.4 Gas foaming pressure release preformed on 05/14/2018 78

B.5 Gas foaming pressure release preformed on $05 / 15 / 2018$

C.1 Five inverted optical microscope images of Alizarin Red S-stained dPBS-soaked scaffold \#1 cryosections taken at $4 \mathrm{x}$ magnification

C.2 Five inverted optical microscope images of Alizarin Red S-stained dPBS-soaked scaffold \#2 cryosections taken at $4 \mathrm{x}$ magnification

C.3 Five inverted optical microscope images of Alizarin Red S-stained ODM-soaked scaffold \#1 cryosections taken at $4 \mathrm{x}$ magnification

C.4 Five inverted optical microscope images of Alizarin Red S-stained ODM-soaked scaffold \#2 cryosections taken at $4 \mathrm{x}$ magnification

C.5 Five inverted optical microscope images of Alizarin Red S-stained ODM-soaked scaffold \#3 cryosections taken at $4 \mathrm{x}$ magnification

C.6 Five inverted optical microscope images of Alizarin Red S-stained ODM-soaked scaffold with osteoblast action \#1 cryosections taken at $4 \mathrm{x}$ magnification

C.7 Five inverted optical microscope images of Alizarin Red S-stained ODM-soaked scaffold with osteoblast action \#2 cryosections taken at $4 \mathrm{x}$ magnification .86

C.8 Five inverted optical microscope images of Alizarin Red S-stained ODM-soaked scaffold with osteoblast action \#3 cryosections taken at 4x magnification 


\title{
LIST OF ABBREVIATIONS
}

\author{
ANOVA - Analysis of Variance \\ DAQ - Data Acquisition Board \\ dPBS - Dulbecco's Phosphate Buffered Saline \\ ECM - Extracellular Matrix \\ NI - National Instruments \\ Ob - Osteoblasts \\ OCT - Optimum Cutting Temperature \\ ODM - Human Osteoblast Differentiation Media \\ PID - Proportional Integral Derivative Controller \\ PGA - Poly(Glycolic Acid) \\ PLA - Poly(Lactic Acid) \\ PLGA - Poly (Lactic-co-Glycolic Acid) \\ SEM - Scanning Electron Microscope
}




\section{Chapter 1}

\section{INTRODUCTION}

Diseases and injuries can cause trauma to tissues within the body that require reparations or replacement. Tissue regeneration and replacement is commonly achieved through tissue engineering. Tissue engineering is the combination of cells grown in vitro with porous biocompatible scaffolds. There are multiple different methods of scaffold creation along with multiple different biomaterials used to create these scaffolds. Different combinations of scaffold creation methods and materials better suit different tissue engineering needs.

This study investigates gas foaming as a novel method for creating Poly (Lactic-coGlycolic Acid) (PLGA) scaffolds for bone tissue engineering. Gas foaming is not a commonly used scaffold creation method, yet it holds promise due its ability to create highly porous scaffolds when combined with particulate leaching. It also avoids the use of organic solvents and it can easily be modified to create different intentional results (Harris, Kim, \& Mooney, 1998).

\subsection{Purpose of Thesis}

This thesis serves to investigate the ability to use gas foaming to create PLGA scaffolds

for bone tissue engineering and to determine whether these PLGA scaffolds in the presence of osteoblasts better replicate the mechanical stiffness of native trabecular bone than these PLGA scaffolds alone. 


\subsection{Scope of Thesis}

There were five main steps involved in the successful completion of this thesis.

The first step was to build and test the gas foaming apparatus and control system. This involved creating a reproducible method of releasing gas pressure from the gas foaming chamber. Two different methods were focused on and detailed in Section 3.1. Pressure over time graphs during pressure release were recorded and analyzed, and Scanning Electron Microscope images were taken of PLGA scaffolds made via both methods and compared to determine which release method was most suitable for this study.

The second step was to fabricate the PLGA scaffolds via gas foaming. PLGA flakes and deionized salt were ground down and sieved to obtain a controlled range of 63 to 250 micron diameter particles. The PLGA and salt particles were then weighed out to achieve a mass ratio of $80 \%$ salt and 20\% PLGA. The salt and PLGA were combined and pressed into the desired scaffold shape. This pressed pellet was then subjected to 800 psi of carbon dioxide gas for 22 hours. The release of the gas after 22 hours then allowed the PLGA particles to fuse together. The salt was then leached out in water for 24 hours, yielding a porous PLGA scaffold.

The third step was to culture osteoblasts for deposition and culture on the scaffolds. Aseptic procedures were strictly followed to ensure sterility as one vial of one million osteoblasts was expanded to 9 flasks with approximately 1 million osteoblasts each. The osteoblasts were stripped from these flasks and deposited onto scaffolds soaking in human Osteoblast Differentiation Medium (ODM) in a 48-well plate. One million osteoblasts per scaffold are deposited onto 7 scaffolds soaking in ODM. There are also 7 scaffolds soaking in ODM that do 
not have osteoblasts deposited on them, 7 scaffolds soaking in Dulbecco's Phosphate-Buffered Saline (dPBS), and 4 scaffolds that were left dry in the 48 -well plate.

The fourth step was to determine the Young's modulus of each scaffold via compression testing. Four of each scaffold treatment group were used in compression testing. Compressive load versus extension data for each scaffold were recorded. These data were used to determine the maximum load recorded, the load at the yield point, and the Young's modulus of each scaffold. These obtained values were compared to the literature values seen in native trabecular bone.

The fifth and final step was to test that the scaffolds that had osteoblasts deposited on them showed signs of calcification. Osteoblasts will deposit and calcify extracellular matrix, so calcium deposits were used as a measure of osteogenic action. This was completed by freezing the scaffolds in optimum cutting temperature (OCT) gel and using a cryostat to obtain cryosections of each scaffold. These cryosections were then stained with Alizarin Red S, which stains calcium-containing components red. These cryosections were viewed under an inverted optical microscope and analyzed to show that the amount of calcium-containing components on the scaffolds increased in the presence of osteoblasts, thereby demonstrating osteogenesis had occurred on gas foamed PLGA scaffolds.

When all five of these steps were completed, the thesis project was considered complete. 


\section{Chapter 2}

\section{LITERATURE REVIEW}

Bone tissue engineering first started gaining popularity in the second half of the 1990s, as it offered a novel approach to bone repair (Liu \& Ma, 2004). At the time, calcium supplements, autografts, and allografts were common treatments and procedures prescribed to those in need of bone tissue repair or replacement. However, each of these approaches have significant limitations. The body does not make calcium naturally, but instead acquires it through diet. If one's diet is low in calcium, bone mechanical strength can be compromised leading to injury or disease. Calcium supplements are intended to add additional calcium to the body to strengthen, or increase the stiffness of one's bones. However, the effectiveness of calcium supplements is highly debated. The supplements are reported to slow bone resorption, but not stop or reverse bone resorption. Calcium supplements have also been rumored to increase the likelihood of adverse cardiovascular effects (Shin \& Kim, 2015). An autograft is bone tissue taken from one part of the patient's body that is then transplanted to another part of their body to enable the repair of compromised bone tissue. However, autografts have been shown to lead to pain, infection, scarring, and blood loss (Polo-Corrales, Latorre-Esteves, \& Ramirez-Vick, 2014). An allograft is bone tissue that is taken from one patient and implanted into another. Allografts have the risk of carrying infectious agents (Polo-Corrales et al., 2014). Calcium supplements, autografts, and allografts can all help in reducing bone resorption, but tissue engineering shows more promise in mitigating the effects of damage in bone tissue. 
Tissue engineering requires a scaffold on which to grow cells and there are numerous methods to create these scaffolds. This study utilized gas foaming to create Poly Lactic-coGlycolic Acid (PLGA) scaffolds. Using gas foaming to create synthetic polymer scaffolds first started gaining traction in 1998 in the Mooney Lab documented in the article "Open pore biodegradable matrices formed with gas foaming" (Harris et al., 1998). Mooney, et al. created porous PLGA scaffolds by pressing pellets of 85:15 PLGA and salt at various salt:PLGA ratios and subjecting the pellets to 800 psi of carbon dioxide gas for 48 hours. The salt was then leached from the pellets by soaking in deionized water for 48 hours. This created porous PLGA scaffolds that smooth muscle cells readily adhered to. The porosity of the scaffolds could be controlled by the ratio of salt to PLGA used and the salt particle size. These findings demonstrated gas foaming as a viable process for creating polymer scaffolds for threedimensional tissue growth.

Two of the more common methods of tissue scaffold synthesis are electrospinning and solvent casting. Electrospinning creates polymeric fibers on the order of hundreds of nanometers in diameter via electric force and overlays them to mimic extracellular matrix components and geometry (Bhardwaj \& Kundu, 2010). Electrospun scaffolds have high surface to volume ratio (an advantageous characteristic for cell retention), have high porosity, and can be easily manipulated to achieve a wide range of geometries. However, electrospinning does not replicate the bulk morphology of trabecular bone to the same extent as gas foaming. Solvent casting involves dissolving a polymer in a solvent, mixing in salt particles, and pouring this solution into a mold. The solvent is removed in a vacuum and the salt is leached out in water to create a polymeric tissue scaffold (Liao et al., 2002). Solvent casting replicates the bulk morphology of trabecular bone tissue well, but residues of organic solvents that can remain in the polymer 
scaffolds after solvent casting have been a cause for concern (Harris et al., 1998). Gas foaming manages to model the bulk morphology of trabecular bone without the use of solvents. Figure 2.1 shows Scanning Electron Microscope (SEM) images of scaffolds created by each of the methods described compared to an SEM image of healthy adult trabecular bone.

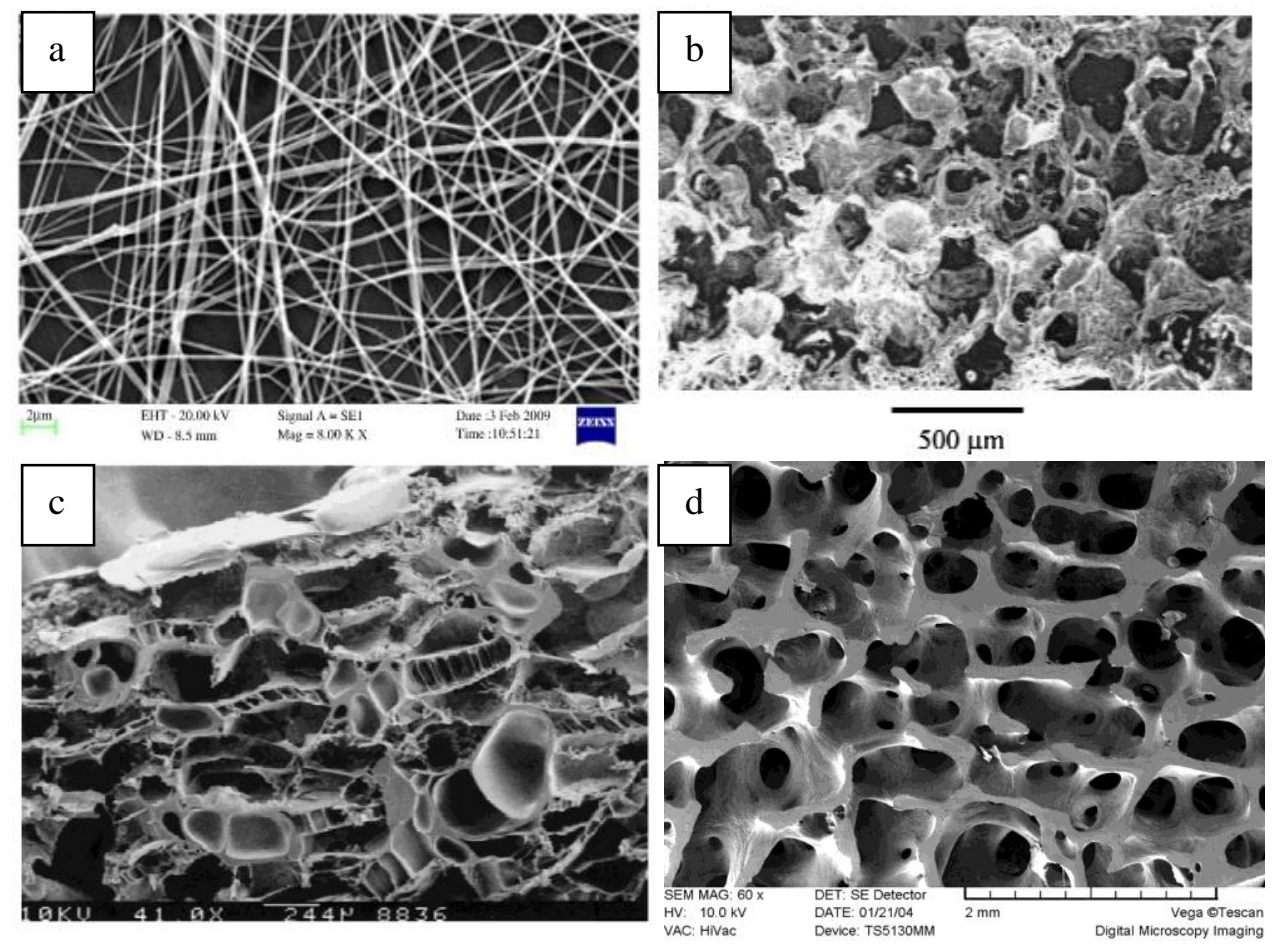

Fig 2.1 Scanning electron microscope (SEM) images of tissue scaffolds created via a) electrospinning (Bhardwaj \& Kundu, 2010), b) solvent casting and particulate leaching (Liao et al., 2002), and c) gas-foaming and particulate leaching (Harris et al., 1998) compared to d) an SEM image of healthy trabecular bone (Hansma, 2004)

The remainder of the literature review will cover information necessary to understand the various facets within this project.

\subsection{Poly (Lactic-co-Glycolic Acid)}

Poly (Lactic-co-Glycolic Acid) or PLGA is a common synthetic polymer used as a scaffold material for bone growth due to its biocompatibility, controllable degradation rate, and ability to be easily modified. PLGA is FDA-approved and can be exported universally due to its 
lack of animal-derived products (Avgoustakis, 2005). PLGA scaffolds and composites (PLGA combined with other biocompatible materials) have been used in a variety of tissue engineering applications as a substrate for cell deposition and ECM formation. Numerous studies have demonstrated that osteoblasts exhibit great adhesion and proliferation properties on PLGA (Palin, Liu, \& Webster, 2005). The most common applications of PLGA in bone tissue engineering on the market are orthopedic implants, drug delivery, interference screws, suture anchors, bone plates, and anterior cruciate ligament reconstruction (Gentile, Chiono, Carmagnola, \& Hatton, 2014).

PLGA is a copolymer between polylactide (PLA) and polyglcolide (PGA), and the ratio of lactide to glycolide determines the material's mechanical properties. PLGA can come in two different crystalline forms: semicrystalline and amorphous (Harding, 2018). Semicrystalline polymers have a highly-ordered molecular structure. They remain solid until they reach their melting points where they rapidly become a liquid of low viscosity (RTP, 2016). Amorphous polymers, on the other hand, do not show any sign of crystallinity (Ngai, Floudas, Plazek, \& Rizos, 2002). The molecular structures of semicrystalline and amorphous polymers are illustrated in Figure 2.2. Amorphous PLGA polymers are suitable for biomedical applications, as they provide a more homogeneous distribution of sites for cell integration (Kang, La, \& Kim, 2009). 


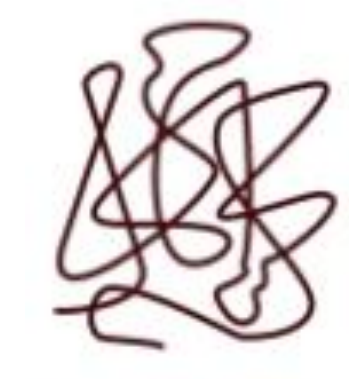

Amorphous

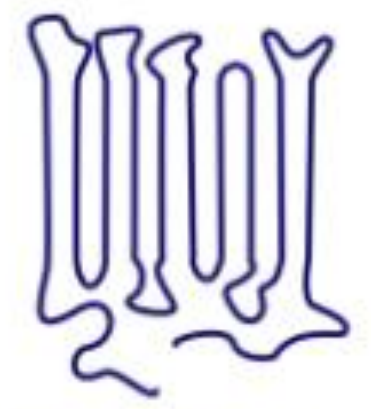

Semicrystalline

Fig 2.2 Visual representation of the molecular organization of amorphous vs. semicrystalline polymers (MacVarish, 2017)

PLA is more hydrophobic than PLGA and PGA is less hydrophobic than PLGA. Therefore, PLGA that is more lacticide than glycolicolide will be more hydrophobic. PLGA degrades faster than both PLA and PGA, with a 50lactide:50glycolide ratio degrading the fastest. Figure 2.3 demonstrates the effect of PLA:PGA ratio on PLGA degradation time.

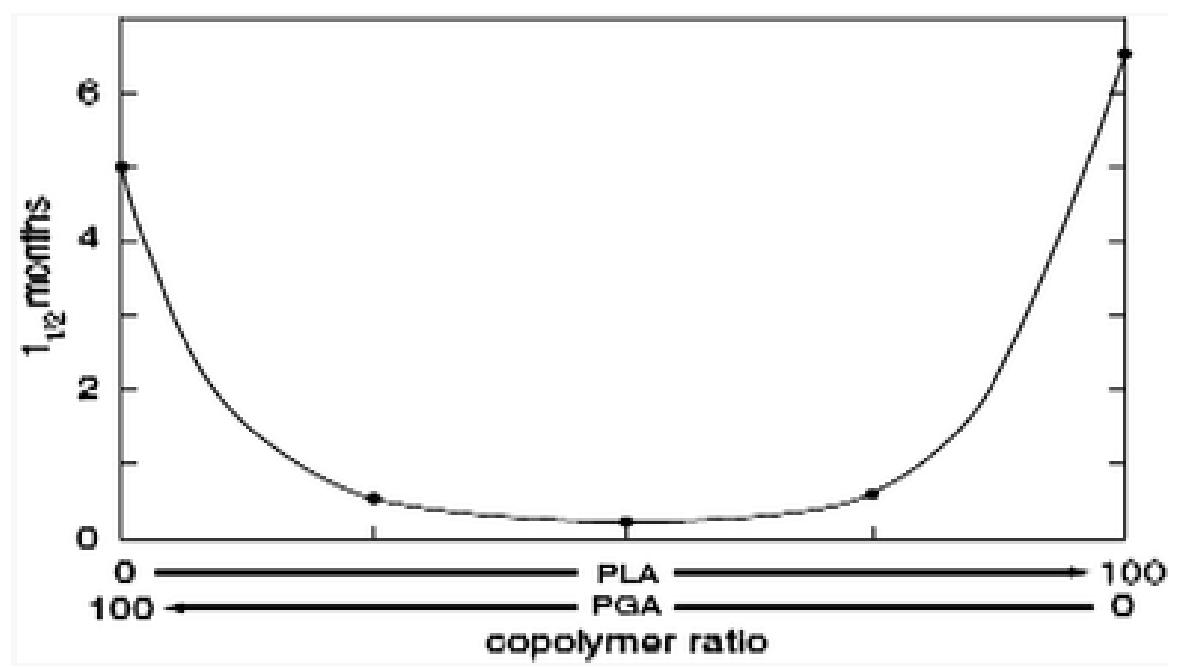

Fig 2.3 Time until polymer is to half of its density after degrading in water based on ratio of PLA to PGA present in PLGA (Felton, 2011)

\subsection{Gas Foaming}

Gas foaming is the process of fusing polymer particles together to create a solid polymer. A polymer that has been pressed into its desired shape is placed into a sealed chamber connected 
to a carbon dioxide tank and a release valve. The pressed polymer pellet is then subjected to high gas pressure with the release valve closed for enough time to allow the polymer to saturate with carbon dioxide gas. By rapidly releasing the pressure from the valve and allowing the pressure inside the chamber to return to ambient conditions, a thermodynamic instability is created within the polymer particles. The carbon dioxide gas then separates from the polymer and the polymer undergoes pore nucleation and growth, a process in which the atoms of the polymer self-organize and the polymer particles expand into each other to create a solid. Once this process is completed, a solid polymer structure is created (Harris et al., 1998).

\subsection{Proportional-Integral-Derivative Controller}

To allow for proper pore nucleation and growth, the rate at which pressure is released from the gas foaming chamber is important. If the carbon dioxide gas is released too slow, the polymer particles will not fuse together. If the carbon dioxide gas is released too fast, the polymer pores will grow too fast and can result in gross defects of the scaffold as shown in Figure 2.4. This is where having a repeatable method of pressure release becomes important. A Proportional-Integral-Derivative Controller (PID) can be used to control the rate at which pressure is released from a gas foaming chamber by controlling the voltage being sent to a positioner on an actuated release valve. In this scenario, a PID would take the pressure reading inside the chamber, compare it to the programmed, desired pressure reading at that point in the pressure release, and attempt to correct the current pressure to the desired pressure. 


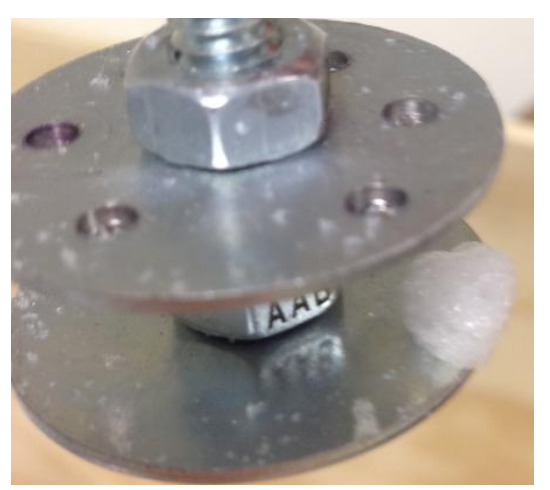

Fig 2.4 An unsuccessful PLGA scaffold creation attempt where pressure was released too rapidly from the gas foaming chamber

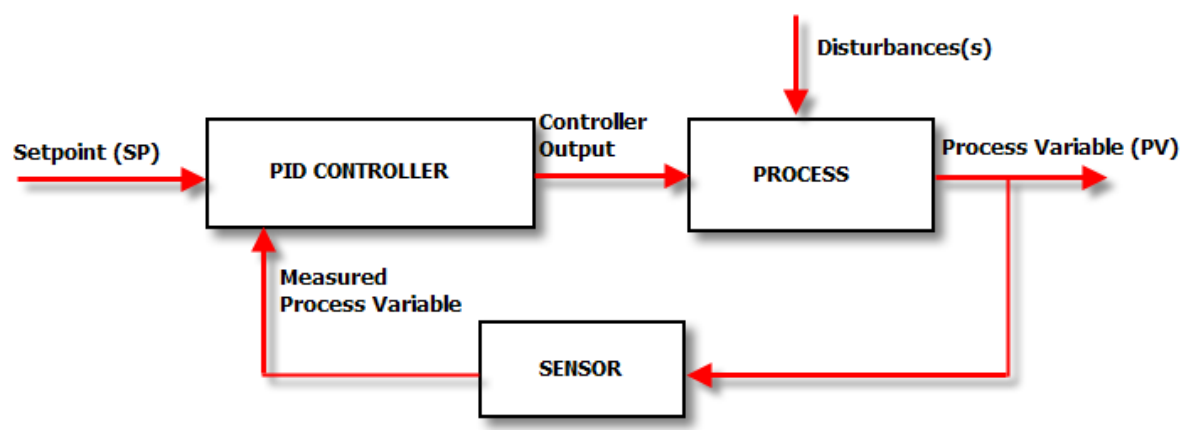

Fig 2.5 Basic block diagram of a process controlled by a PID(Knospe, 2006)

A PID regulates a process by the steps shown in Figure 2.5. The most essential parts to understanding this block diagram are the set point and process variable. The set point is the value we want the process to be at a given time. The PID controller compares the value of the set point to the value of the process variable, the actual measured value of the process at a given time. The PID then applies three calculations to the process variable to calculate how much to change the process variable to achieve the set point. The three calculations are called modes of control and they are proportional, integral, and derivative. The three modes of control are all added together. Therefore, the overall changes the PID makes to the process is the sum of the proportional, integral, and derivative modes of control. The proportional mode of control multiplies the difference between the set point and process variable by a determined constant, Kp. The integral mode of control sums all of the values of error that have happened so far in the process and 
multiplies them by a determined integral constant, Ki. A larger integral constant should bring the process variable to the set point faster since it will be evaluating the trend the process variable has been taking further back in the process. The derivative mode of control predicts what is about to happen by projecting the current rate of change into the future. It also has a determined derivative constant, $\mathrm{Kd}$ (Knospe, 2006). Getting the process variable to follow a given set point involves adjusting $\mathrm{Kp}, \mathrm{Ki}$, and $\mathrm{Kd}$.

\subsection{Scanning Electron Microscopy}

A Scanning Electron Microscope (SEM) allows people to view samples at a resolution of 10-100nm (Savage, 2017). To put this in perspective, the human eye has a natural resolution limit of $100 \mu \mathrm{m}$ and optical microscopes have a resolution limit of $0.25 \mu \mathrm{m}$. SEMs work by bombarding a sample surface with electrons and detecting the secondary electrons that are scattered off the material surface. These secondary electrons are interpreted to produce a grayscale image of the sample's surface. A common problem with SEMs is that a nonconductive sample surface can become overcharged by a buildup of electrons resulting in bright streaks across the surface of images (Ammrf, 2014). To prevent overcharging, a thin layer of gold is often sputtered onto a sample's surface to act as a conductive surface, thereby decreasing the accumulation of static electric fields (Höflinger, 2013).

\subsection{Osteoblasts}

Osteoblasts are the cells responsible for the deposition of bone matrix. The bones in the human body are constantly undergoing resorption and restoration. Osteoclasts disintegrate bone matrix via the release of hydrogen ions which converts water and carbon dioxide into 
bicarbonate, an acid that dissolves bone (Väänänen, Zhao, Mulari, \& Halleen, 2000). Osteoblasts then secrete extracellular matrix (ECM) which is composed of collagen-1 fibrils and noncollagenous proteins in bone. The ECM is then mineralized by matrix vesicles containing calcium-phosphate crystals secreted by the osteoblasts (Hasegawa et al., 2017).

The process of bone formation is called osteogenesis, and there are two distinct osteogenic processes, intramembranous ossification and endochondral ossification. The primary difference between these two forms of bone formation is that endochondral ossification utilizes cartilage as a precursor for bone whereas intramembranous ossification does not. The process of osteogenesis starts when mesenchymal stem cells cluster together and begin replicating. This cluster is called a nidus, and when a nidus is completely formed the mesenchymal stem cells stop replicating and begin experiencing morphological changes (Figure 2.6). These morphological changes cause the mesenchymal stem cells in the nidus to become osteoblasts. The osteoblasts begin laying down collagen-1 and extracellular matrix proteins (Brighton \& Hunt, 1997) and then secrete matrix vesicles containing calcium-phosphate crystals. It is believed that these calcium-phosphate crystals are formed by the influx of calcium and phosphate ions across the matrix vesicle's plasma membrane. The crystals form on the inside of the matrix's plasma membrane and then rip through the plasma membrane, resulting in the deposition of mineralized nodules on the extracellular matrix of bone (Hasegawa et al., 2017). This process is illustrated in Figure 2.7. The growth of osteoblasts in-vitro has been cited as limited in its capacity to replicate the organization and density of osteoblasts in-vivo, which has been theorized to be due to the lack of inhibitory cytokines secreted by osteoblasts which indicate the site for calcification and growth from osteoblasts (Blair et al., 2017). 


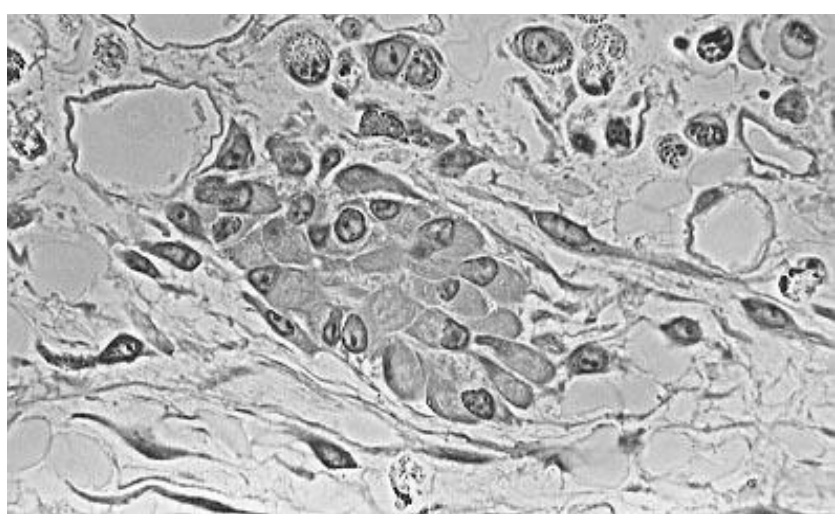

Fig 2.6 Light micrograph image of mesenchymal stem cells clustering to form a nidus (Brighton \& Hunt, 1997)
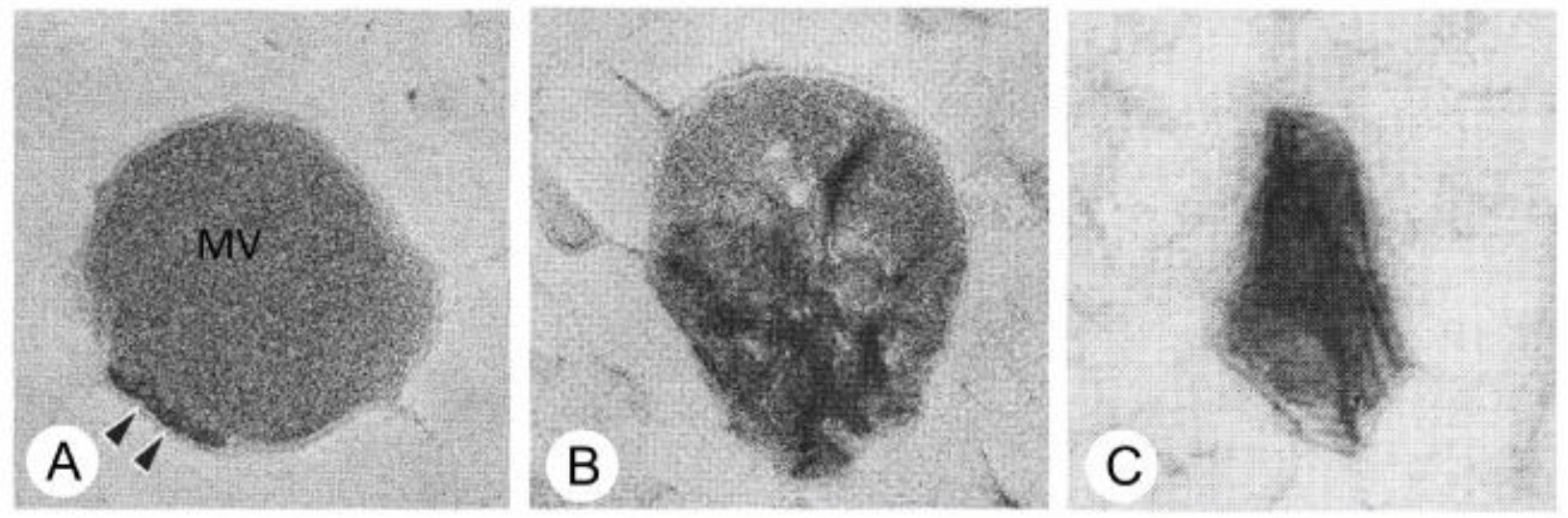

Fig 2.7 Transmission Electron Microscope images of a matrix vesicle secreted from an osteoblast during osteogenesis. a) shows the beginning of crystallization on the inner plasma membrane, b) shows the growth of calcium-phosphate crystals within the vesicle, and c) shows the crystals tearing through the plasma membrane (Hasegawa et al., 2017)

It has previously been determined that the diameter of an average osteoblast is between $15 \mu \mathrm{m}$ and $20 \mu \mathrm{m}$ and pits in scaffold surfaces between $30-40 \mu \mathrm{m}$ in diameter best promote ossification (Tanaka-Kamioka, Kamioka, Ris, \& Lim, 1998). Pores larger than the 30-40 $\mu \mathrm{m}$ diameter range are still desirable as there will be smaller pits within these larger pores that do fall within this range. Therefore, scaffolds with pores at least $30 \mathrm{um}$ in diameter would be suitable for osteoblast attachment, growth, and calcium deposition onto the scaffold. 


\subsection{Compression Testing}

Compression testing is a mechanical testing process that yields real-time data of compressive load over extension, the distance a compression platen travels from its start position, of materials tested. It involves two stainless steel plates, known as compression platens, that the material sits between, and as the platens slowly get closer together, the compressive load exerted on the material is recorded. Other material properties such as stress, strain, and Young's modulus can be calculated from the recorded data. The Young's modulus is a measure of the stiffness of a material (Rho, Ashman, \& Turner, 1993), and it is often calculated as stress over strain in the linear portion of a stress-strain diagram, as shown in Figure 2.8.

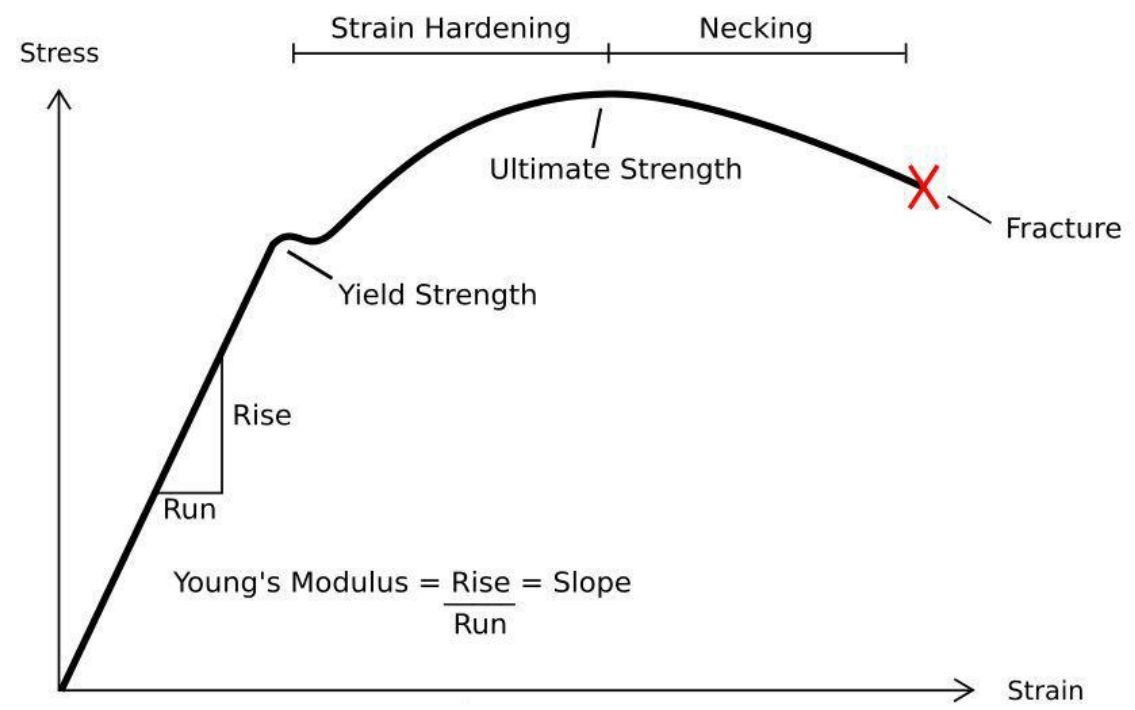

Fig 2.8 Example stress-strain diagram (Figari, 2015)

\subsection{Cryosectioning}

Cryosectioning is the process of creating slices of a frozen sample embedded in clear optimum cutting temperature (OCT) gel. This process is commonly used to create sections of tissue for observing under a microscope. The tissue sample is first embedded into a well of OCT gel and then frozen instantaneously by being submerged in liquid nitrogen. This frozen sample is 
kept in a freezer for 48 hours prior to cryosectioning. The sample is then clamped into place in a cryostat and moved past a sharp blade that creates thin slices of tissue samples. These samples are fixed onto microscope slides to be observed under the microscope of choice.

\subsection{Alizarin Red S}

Alizarin Red S is a histochemical stain that appears red in the presence of calcium. Alizarin Red S can be added to frozen tissue slices to stain calcified areas red. The presence of calcium staining can easily be viewed under an optical microscope (Figure 2.9). When applying Alizarin Red S to a cryosectioned sample, the sample is submerged in Alizarin Red S for three minutes on a microscope slide and preserved by being dipped in acetone and xylene (Byon et al., 2011).

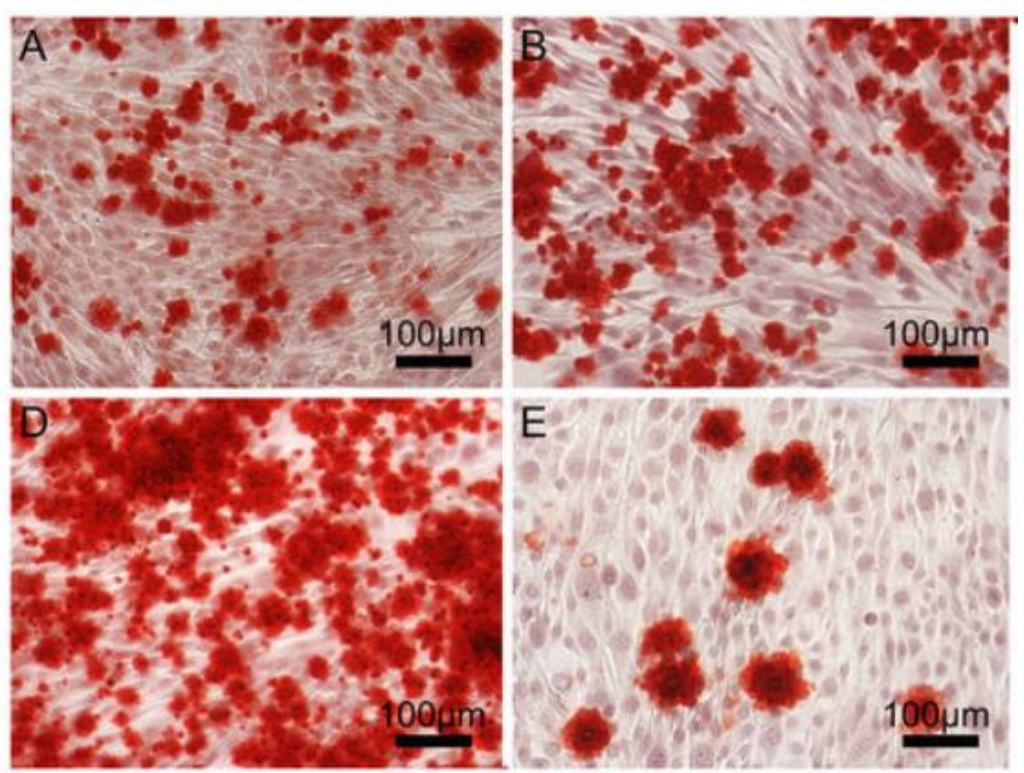

Fig 2.9 Alizarin Red S staining of calcium deposits from pre-osteoblast MC3T3-E1s (Li et al., 2016) 


\section{Chapter 3}

\section{MATERIALS AND METHODS}

There were five essential steps taken to complete this project. An optimal gas foaming method for Poly(Lactic-co-Glycolide) (PLGA) scaffold fabrication had to be determined. PLGA scaffolds had to be created using this gas foaming method. Osteoblasts had to be cultured and deposited on to the PLGA scaffolds. Compression testing of PLGA scaffolds that had osteoblasts grown on them and control treatment groups had to be performed. Lastly, PLGA scaffolds that had osteoblasts grown on them and control treatment groups had to be cryosectioned and stained to test for viability. The process described in this chapter can be viewed as numerical steps in Appendix E.

\subsection{Scaffold Fabrication}

First, we created the PLGA scaffolds and determined the optimal gas foaming procedure in the process. The primary steps involved in this process were milling, pressing, gas foaming, and salt leaching. Milling is the process of grinding a material into a powder. Pressing involves applying pressure to the milled powder in a die to form a shaped pellet. Gas foaming is the process of subjecting the pressed pellet to carbon dioxide gas at a high pressure and releasing the gas to allow the PLGA particles to fuse together. Lastly, salt leaching entails soaking the gasfoamed pellet in deionized water such that the salt diffuses out, leaving a porous PLGA scaffold.

Ultra Epsom medium grain premium salt crystals and Resomer ester-terminated 85 lactide: 15 glycolide PLGA with a molecular weight of 190,000-240,000 g/mol were used for scaffold fabrication. We chose Ultra Epsom salt for its high level of purity and ability to be 
easily dissolved in water (Ultra Epsom, 2010). We chose the Resomer PLGA for a few reasons. For one, the lactide-to-glycolide ratio has been shown to work in past research (Harris et al., 1998). The Resomer PLGA is also described as being made for bioabsorbable implantable devices, which would be a desirable material attribute if we ultimately used this method of scaffold fabrication for bone replacement therapy. The Resomer PLGA has an amorphous molecular structure which is desirable for tissue engineering due to having a more homogeneous distribution of sites for cell integration (Kang et al., 2009). It has a degradation time of approximately 9 months and was stored in a freezer when not in use.

The salt was ground with a $550 \mathrm{~mL}$ Eisco Labs porcelain mortar and pestle and the powder was sieved in the American Educational screen sieves set (Fig 3.1) into particles with diameters between 250 and 63 microns (mesh sizes 60 and 230). The salt was then collected in a plastic tupperware, and the mortar and sieves were thoroughly cleaned. The PLGA was then also milled and sieved into the same size particles. These PLGA particles were collected into a separate plastic tupperware. Conicals were filled with $0.8 \mathrm{~g}$ of salt and $0.2 \mathrm{~g}$ of PLGA, such that each conical represented the materials necessary for one pressed pellet. The pellets were designed to be one gram each and made of $80 \%$ salt and 20\% PLGA by weight. This ratio was used to achieve a moderately porous scaffold when the salt is leached out. More salt was not used to ensure the PLGA particles would not be too far apart to fuse in the gas foaming process. The conicals were then vortexed to thoroughly mix the salt and PLGA particles.

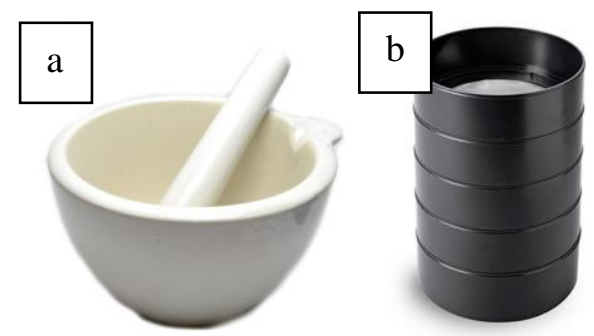

Fig 3.1 a) Eisco Labs porcelain mortar and pestle b) American Educational screen sieves set 
Once the salt and PLGA were ground and divided up into their proper proportions, the materials were ready to be pressed into pellets. The pellets were made one at a time. The contents of a conical with $0.8 \mathrm{~g}$ of salt and $0.2 \mathrm{~g}$ of PLGA were carefully scooped into a $5 \mathrm{~mm}$ Type KB Precision die (Figure 3.2a). We placed the die under a $2 \mathrm{kN}$ press (Figure 3.2b) and pressure was manually added to the die by pressing down on the lever arm of the press. The one gram of mixed salt and PLGA powder was now shaped and compacted into a cylindrical pellet (Fig 3.1.2c) inside of the die and carefully removed. The pellets have a diameter of $5 \mathrm{~mm}$ and an average height of $3 \mathrm{~mm}$.

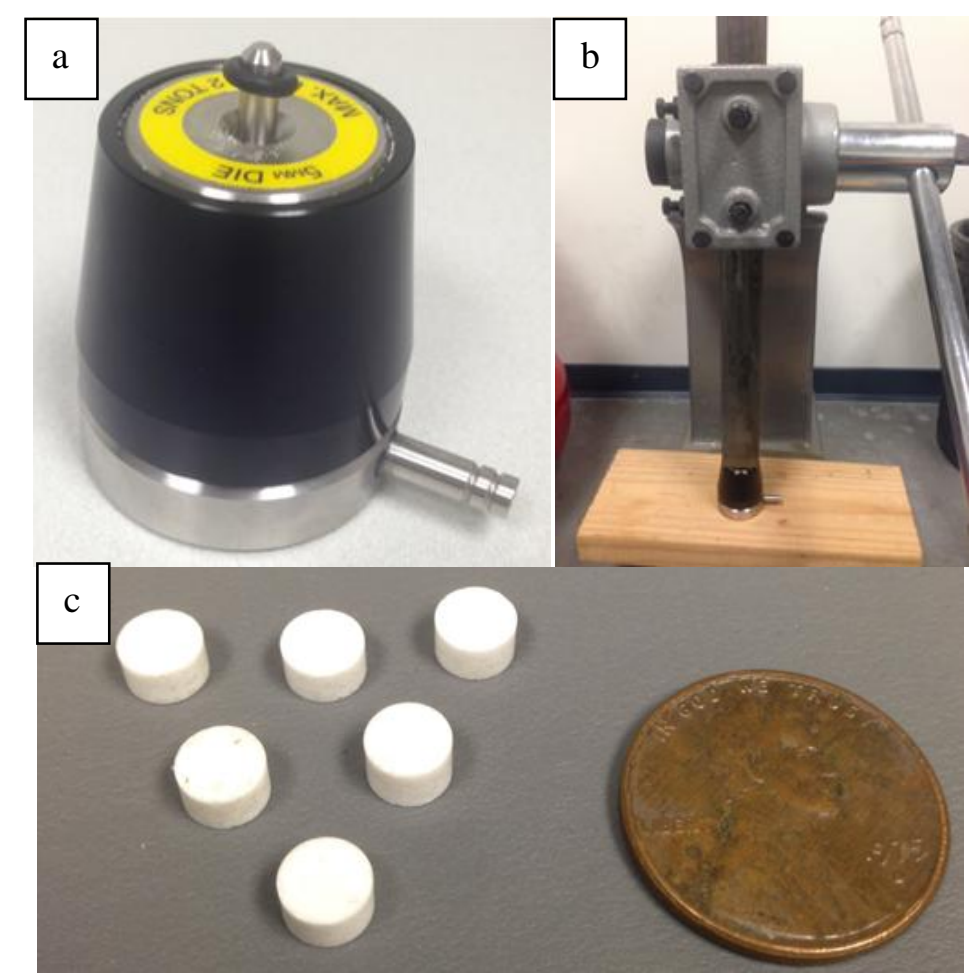

Fig 3.2 a) $5 \mathrm{~mm}$ Type KB Precision die b) Die under $2 \mathrm{kN}$ press c) Pressed pellets of mixed PLGA and salt

We fused the PLGA particles together using gas foaming. Gas foaming fuses PLGA particles in the pressed pellet together by creating a thermodynamic instability as pressure is released from the gas foaming chamber that leads to the nucleation and growth of gas pores inside the PLGA particles (Harris et al., 1998). A pre-existing carbon dioxide pressure vessel on 
campus, which had previously been used in Cal Poly MEDITEC projects, was modified and used for the purpose of gas foaming. First, the carbon dioxide tank was switched from a liquid tank to a gas tank. The setup originally ran through two pressure vessels, one of which could be temperature-controlled and the other had a pressure transducer for reading the pressure within. The gas line to the temperature-controlled vessel had been broken and would leak carbon dioxide gas when run. The plumbing was reconstructed such that the temperature-controlled vessel was skipped. The carbon dioxide tank now ran to a manual needle valve then to the pressure vessel with a pressure transducer. A pipe ran out of the pressure vessel and led to another manual needle valve which led to ambient air. This needle valve was how carbon dioxide gas was released from the pressure vessel. We replaced this needle valve with a Valworx Electric Actuated Stainless Ball Valve with Electronic Positioning System, also called a positioner, because we wanted a more reproducible method of gas pressure release.

The carbon dioxide gas needs to be released in a controlled fashion. Releasing the gas too fast will result in excessive pore nucleation of the PLGA scaffolds. The result is gross scaffold architecture that does not maintain its original cylindrical shape, taking on a 'popcorn'-like appearance due to the excessive and uncontrolled pore expansion. Releasing the gas too slow will yield a lack of pore nucleation in the PLGA scaffold, resulting in a lack of or inefficient fusing of adjacent PLGA particles and the scaffold will easily crumble. To accomplish a controlled gas release, we built a controlled gas release apparatus consisting of a data acquisition board (DAQ) that sends pressure readings from the pressure transducer in terms of AC current to an National Instruments Labview program, where the pressure readings are interpreted, and then the DAQ sends a set DC voltage to the positioner on the actuated release valve. This process is diagrammed in Figure 3.3. The DAQ used is National Instruments' USB-6002 Multifunction I/O 
device. When $0 \mathrm{~V}$ are sent to the positioner, the ball valve is completely closed, and when $10 \mathrm{~V}$ are sent to the positioner, the ball valve is completely open. Each voltage in between $0 \mathrm{~V}$ and $10 \mathrm{~V}$ corresponds proportionally with how open the ball valve is. For example, $5 \mathrm{~V}$ corresponds to a halfway open ball valve. The DAQ reads the pressure inside the gas foaming chamber via a male McMaster standard, 4-20 mA output, 1/4" NPT, 5000 psi pressure transducer. This allows the pressure over time to be recorded and plotted for evaluation purposes. A proportional-integralderivative controller (PID) was implemented and programmed in the National Instruments LabView software to provide a feedback control loop between real-time pressure transducer measurements and the magnitude and duration of valve opening. Multiple gas releases (approximately 75) were performed with the PID, incrementally changing the PID constants by orders of magnitude then fine-tuning inwards until the optimal PID constants were found to get the process variable to follow our predetermined set point of a linear decrease from 800psi to Opsi over 30s. The PID constats were found to be $\mathrm{Kp}=0.056, \mathrm{Ki}=20,000$, and $\mathrm{Kd}=0.44$. These values are confirmed in Appendix A. However, despite the ability of the PID to provide real-time feedback, we found that sending a constant voltage to the actuated release valve yielded reduced variability and resulted in more consistent foaming outcomes. More information on how this particular release method was chosen can be found in Section 4.1. The DAQ was connected via USB to a laptop containing a LabView program that I constructed which allows the user to send a predetermined voltage to the positioner. This program also allows pressure readings over time to be collected and exported to a comma-separated values (CSV) file. 


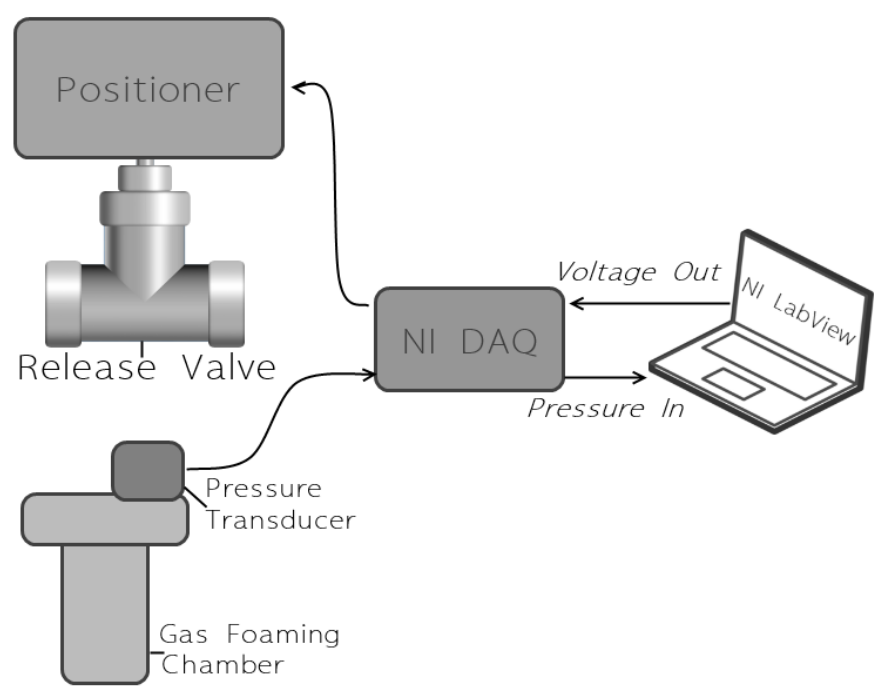

Fig 3.3 Schematic of DAQ setup. DAQ reads pressure in terms of AC current and sends a pressure reading to the NI LabView program. The NI LabView program sends a signal for a specified AC voltage to the DAQ which sends that $\mathrm{AC}$ voltage to the positioner on the release valve.

After the PLGA and salt were pressed into pellets, the lid of the gas foaming chamber was carefully unscrewed to expose the interior of the chamber. Inside there was a removable shelf to place the pressed pellets on (Figure 3.4a \& Figure 3.4b). Six pellets were loaded into the chamber at a time. Once the pellets were on the shelf and carefully lowered into the chamber, the lid was sealed shut. A proper seal and complete closure of the lid is important because it is very easy for a gas leak to occur between the lid and chamber if the lid is not clamped down as tight as possible. We carefully moved the chamber into place and connected it to a gas line attached to a 800psi $\mathrm{CO}_{2}$ tank. The chamber had to remain vertical the entire time so as not to move the pellets around on the shelf. Once it was certain that everything was correctly connected and the release valve was shut, the $\mathrm{CO}_{2}$ tank was opened, filling the gas foaming chamber with 800psi of $\mathrm{CO}_{2}$ gas. The tank was then closed and the scaffolds were left for 22 hours to saturate with the carbon dioxide gas. Once the 22 hours was up, it was time to release the gas pressure from the chamber. 

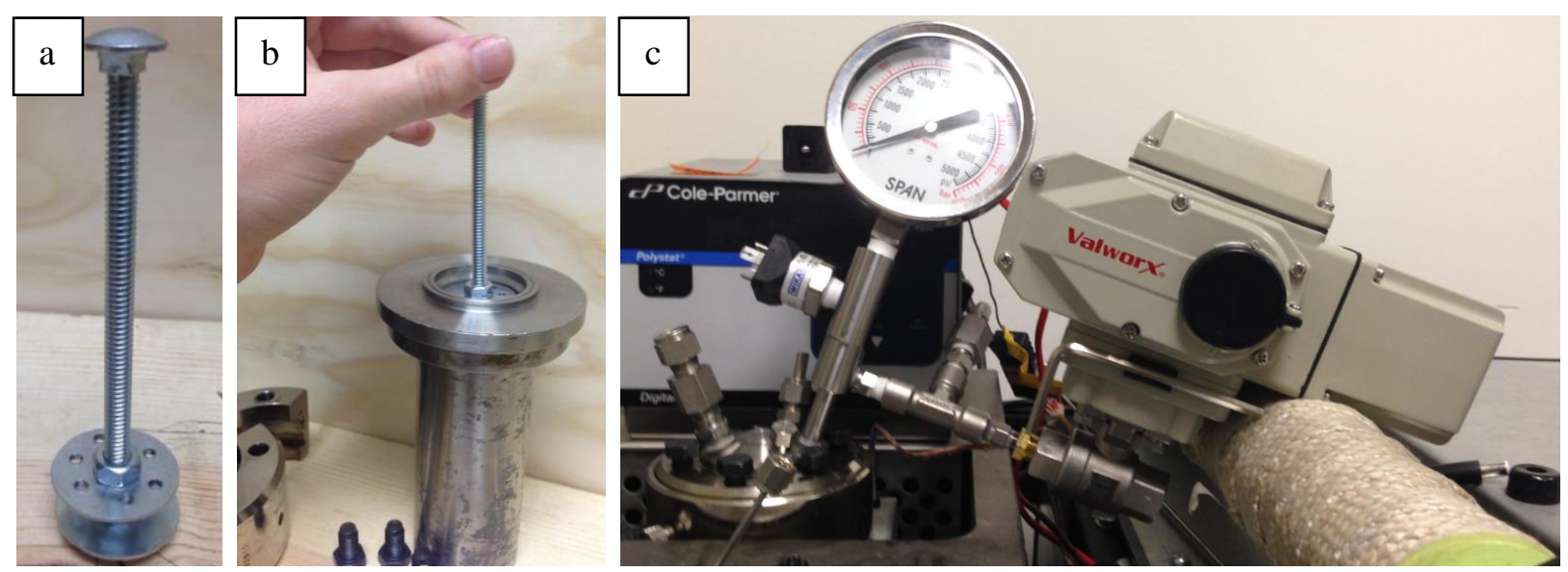

Fig 3.4 a) Removable shelf for scaffolds in gas foaming chamber, b) Demonstration of shelf being removed from the gas foaming chamber, c) Gas foaming chamber and Valworx automated release valve and positioner

Before connecting the actuated valve to the wall, the DAQ was connected via USB to a computer with the LabView software. To calibrate, we sent the LabView program a constant 0V to the actuated release valve. Then, we plugged the actuated release into a wall socket. When the valve was powered on, it received the $0 \mathrm{~V}$ signal and moved to the prescribed shut position. Then, we set the LabView program to send $1.5 \mathrm{~V}$ to the valve. The valve moves accordingly. This was still a closed position, but on the precipice of opening. The LabView program was then set to run. The DAQ sent $2 \mathrm{~V}$ to the actuated release valve, allowing the valve to open up one fifth of the way and the pressure inside the gas foaming chamber returned to ambient conditions within one minute. The graphs of all of the gas foaming pressure releases can be found in Appendix B. A picture of the gas foaming chamber and actuated release valve can be seen in Figure 3.4c.

Once all of the carbon dioxide gas had been released from the chamber, the chamber was disconnected from the gas line. We opened the chamber to reveal PLGA scaffolds that had slightly expanded in volume from their previous pellet state (See Fig 3.6). The scaffolds were then left to soak in deionized water for 22 hours to leach out the salt particles, leaving behind porous PLGA structures. The scaffold fabrication process was now complete. This process was 
repeated until 30 scaffolds had been made. Scaffolds maintained their $5 \mathrm{~mm}$ diameter, but the height increased from approximately $3 \mathrm{~mm}$ to $7 \mathrm{~mm}$.

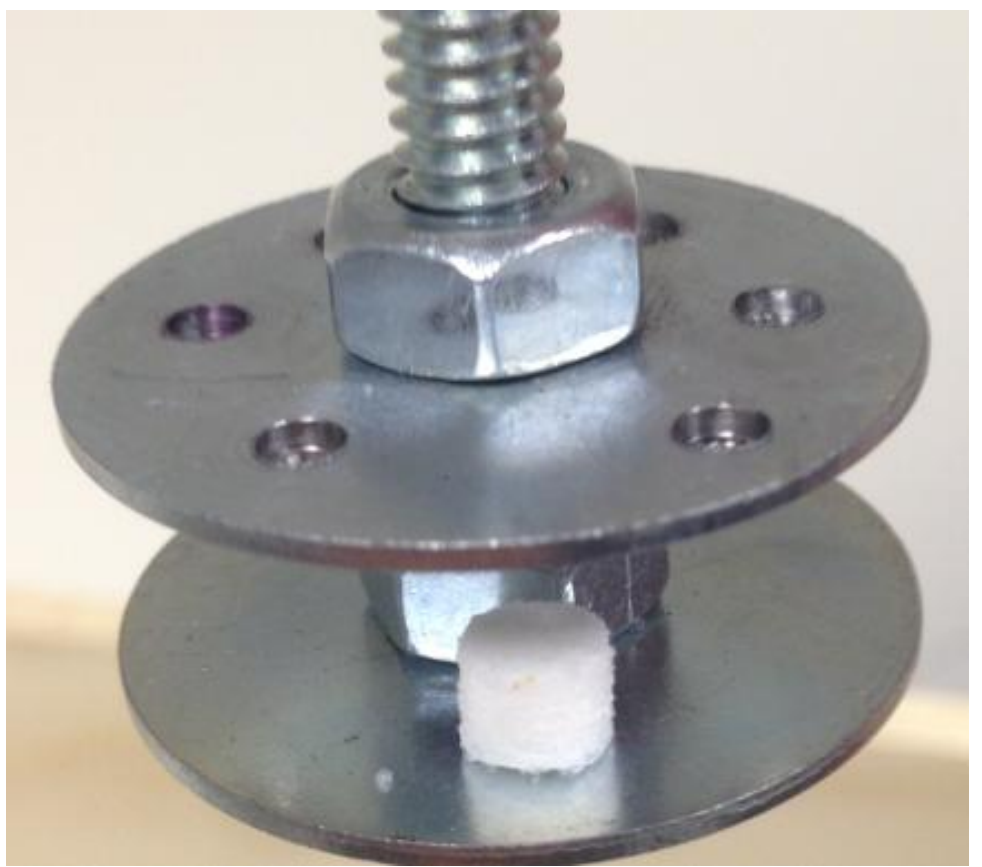

Fig 3.5 A successfully gas foamed PLGA scaffold

\subsubsection{SEM Images of Scaffolds}

To help in determining the most reliable method of gas pressure release for scaffold fabrication, a JEOL JSM-6390 Scanning Electron Microscope (SEM) (Figure 3.7b) was used to take high magnification images of the scaffolds made using the PID-controlled valve and the scaffolds made by sending a constant $2 \mathrm{~V}$ directly to the pressure cylinder release valve positioner. A Denton Vacuum Desk IV (Figure 3.7a) was used to sputter gold on to the surfaces of these scaffolds to prevent the SEM from overcharging the PLGA. The pressure in the Vacuum Desk IV was lowered to $100 \mathrm{mTorr}$ and the sputter time was set to 60 s. Once gold sputtering was complete one scaffold was loaded into the SEM. The scaffold was placed into a locking mechanism that was screwed into place to prevent the scaffold from moving inside the chamber of the SEM (Figure 3.7c). The door to the SEM was closed and the pressure inside was lowered 
to 50 $\mu$ Torr. The SEM's high-voltage cathode was turned on and an image was created in the JEOL JSM-6390 software. Four pictures were taken of each scaffold: one at 30x magnification that attempted to capture the majority of the surface of the scaffold and three at $100 \mathrm{x}$ magnification. The 100x magnification images were taken at the center of the scaffold, just to the left of the image taken at the center of the scaffold, and just to the right of the image taken at the center of the scaffold. Using the software, scalar measurements were taken of various pore widths to help make conclusions about the nature of the scaffold pores created in the two gas foaming methods.
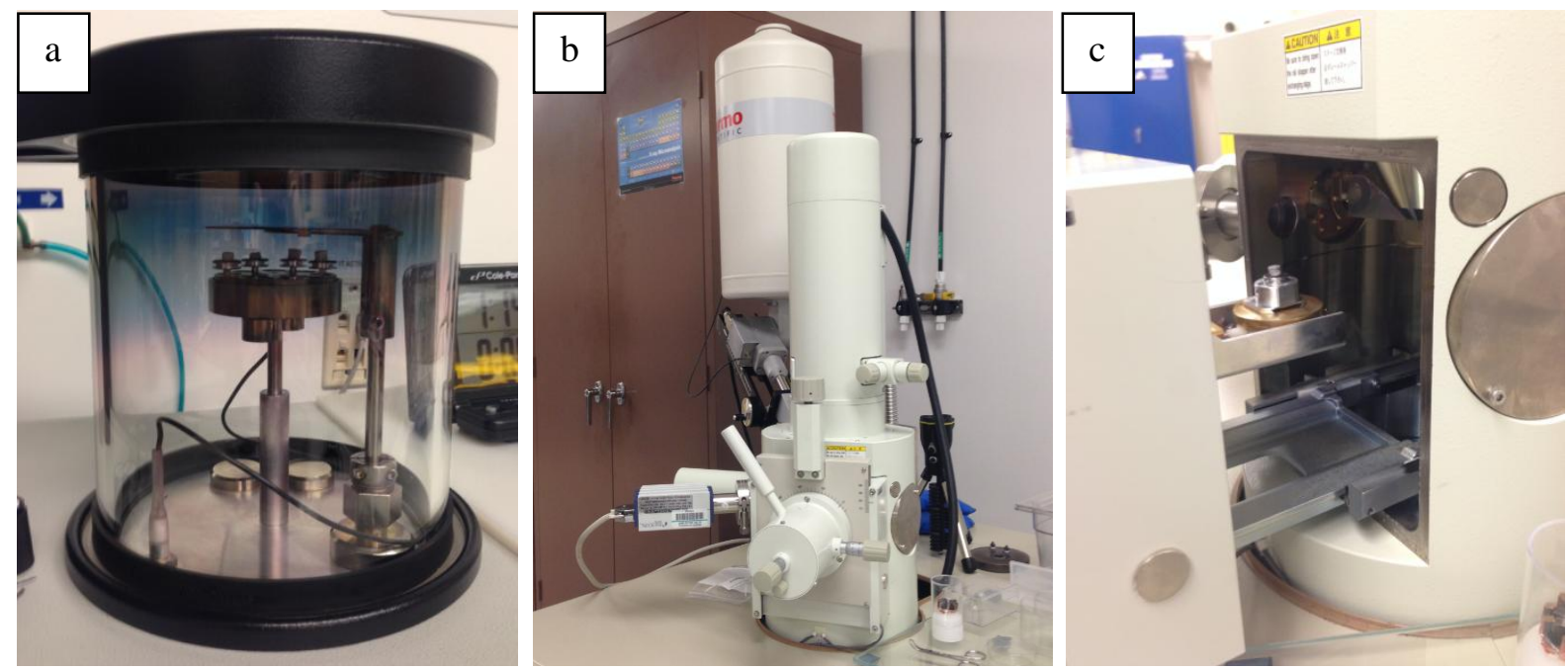

Fig 3.6 a) Denton Vacuum Desk IV gold-sputtering scaffold top surfaces, b) JEOL JSM-6390 SEM, c) loading a gold-sputtered scaffold into the SEM

Along with the qualitative analyses made on these images, Fiji ImageJ was used to make further quantitative analyses. The settings for color threshold were set as shown in Figure 3.8. These settings were determined since they filtered all the pixels that were visibly black and nothing else as shown in Figure 3.9, where a\&d show examples of the original SEM images, b\&e show a mask applied to these images per the settings set in Figure 3.8, and c\&f show that mask being selected for measurement. These same settings were used for each SEM image and 
the filtered pixels were measured and these values were recorded into a Microsoft Excel spreadsheet.

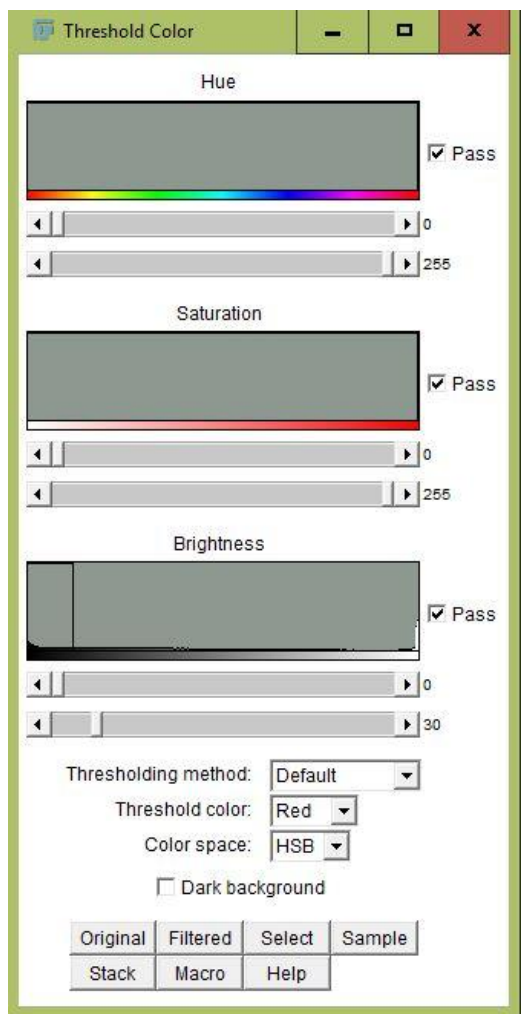

Fig 3.7 Threshold Color settings in Fiji ImageJ for detecting pores in the surface of PLGA scaffolds

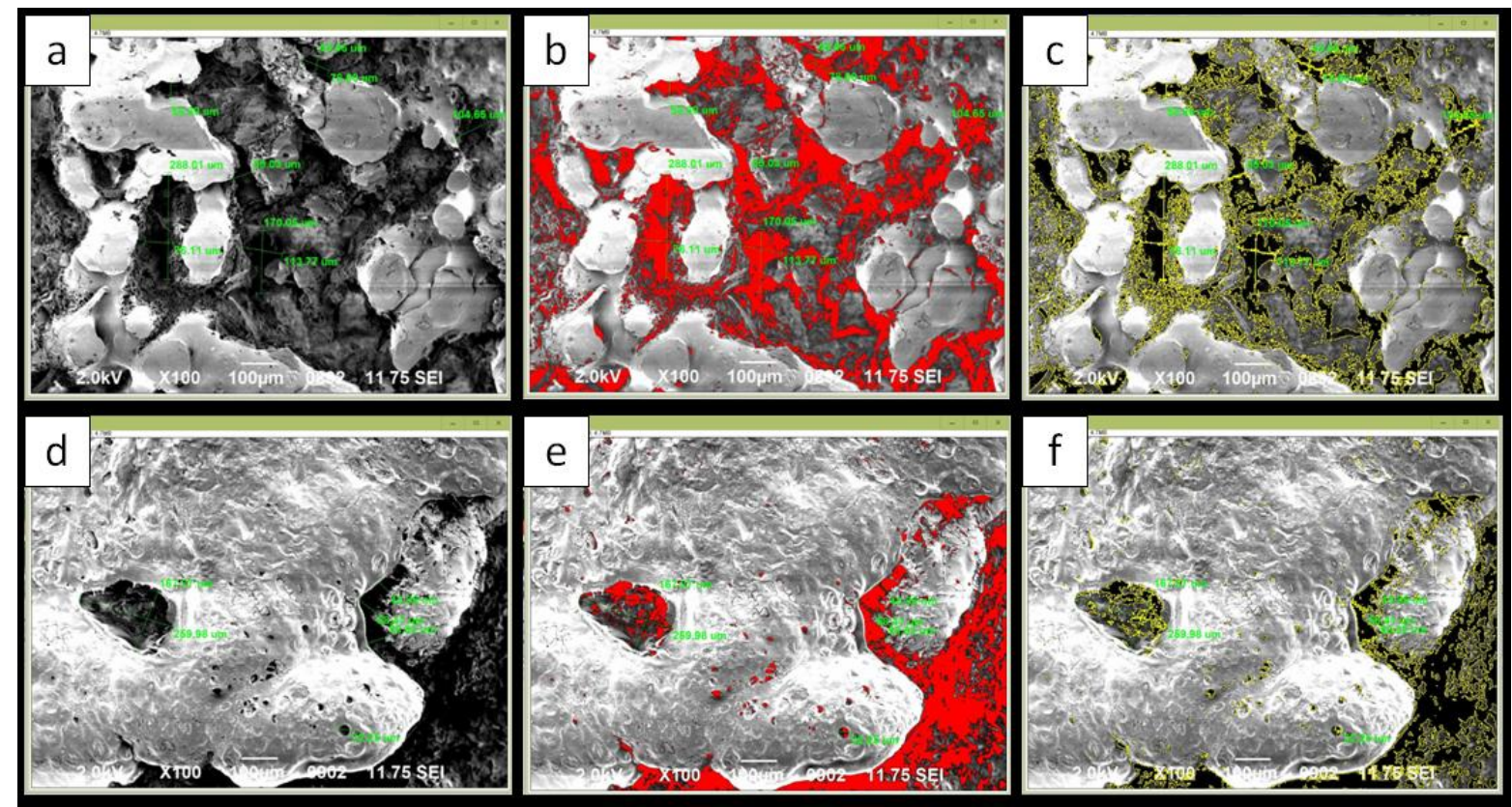

Fig 3.8 a\&d) Original SEM images of scaffolds, b\&e) Filtered SEM images of scaffolds, c\&f) Filtered regions selected for measurements 


\subsection{Cell Culture}

The following section describes the culture and expansion of osteoblasts for deposition on the PLGA scaffolds. I cultured osteoblasts concurrently with scaffold creation since the osteoblasts can be frozen down and later thawed for use at the appropriate time. The major processes I performed were plating, expansion, and freezing of osteoblasts. The osteoblasts used in this experiment were Cell Applications, Inc. primary adult human osteoblasts purchased from Sigma-Aldrich. The osteoblasts were on their second passage upon arrival with less than 500,000 osteoblasts present. They came in a frozen vial of basal medium containing $10 \%$ fetal bovine serum (FBS) and 10\% dimethyl sulfoxide (DMSO). Aseptic procedures were strictly followed to maintain sterility when handling cells and while working under a class II biological safety cabinet.

\subsubsection{Plating}

The osteoblasts were plated in a flask to expand the population of cells. First, $15 \mathrm{~mL}$ of human osteoblast growth medium (Sigma Aldrich) were pipetted into a T-75 flask. The $1 \mathrm{~mL}$ of human osteoblasts was then removed from a portable Dewar, a cryogenic storage container containing liquid nitrogen, and allowed to mostly thaw (a small pellet of ice remaining) in a water bath kept at approximately $37^{\circ} \mathrm{C}$. The osteoblasts were triturated using a pipette and carefully pipetted into the flask containing growth medium. The flask was gently rocked back and forth to evenly distribute the osteoblasts across the bottom of the flask. The flask of osteoblasts was then placed in a humidified incubator maintained at $37^{\circ} \mathrm{C}$ with $5 \%$ carbon dioxide. The growth medium was changed 24 hours later and then every 48 hours. When $60 \%$ confluency, a qualitative term for how much of the flask's surface area was covered by cells, was 
reached, the $15 \mathrm{~mL}$ of growth medium were replaced with $30 \mathrm{~mL}$ of growth medium. Once $80 \%$ confluency was reached, the plating process was complete, and the osteoblasts were ready to be expanded. This process is outlined in Figure 3.10. An image of the osteoblasts at approximately 80\% confluency under an optical microscope is shown in Figure 3.11.

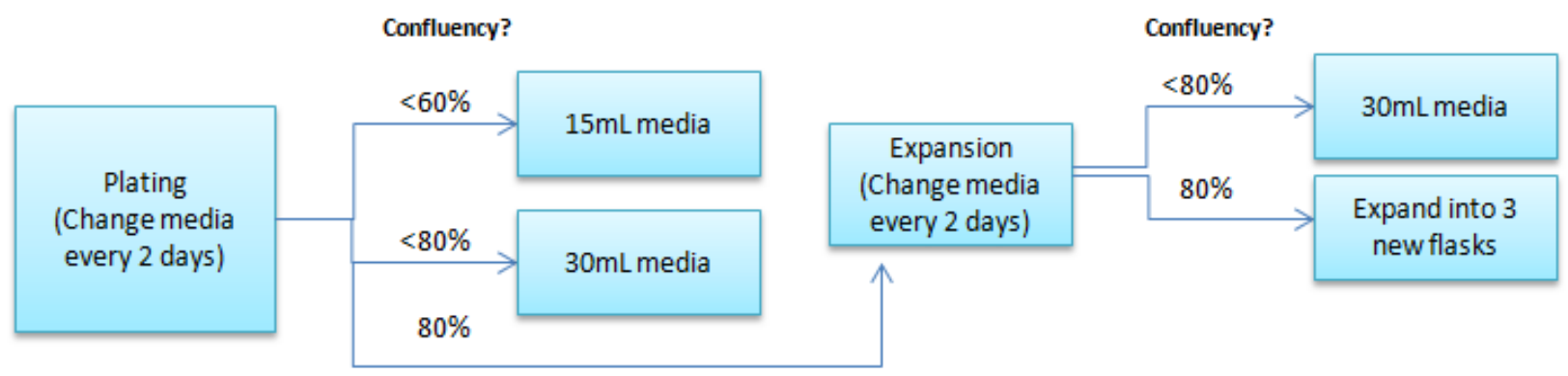

Fig 3.9 Osteoblast plating procedure flowchart

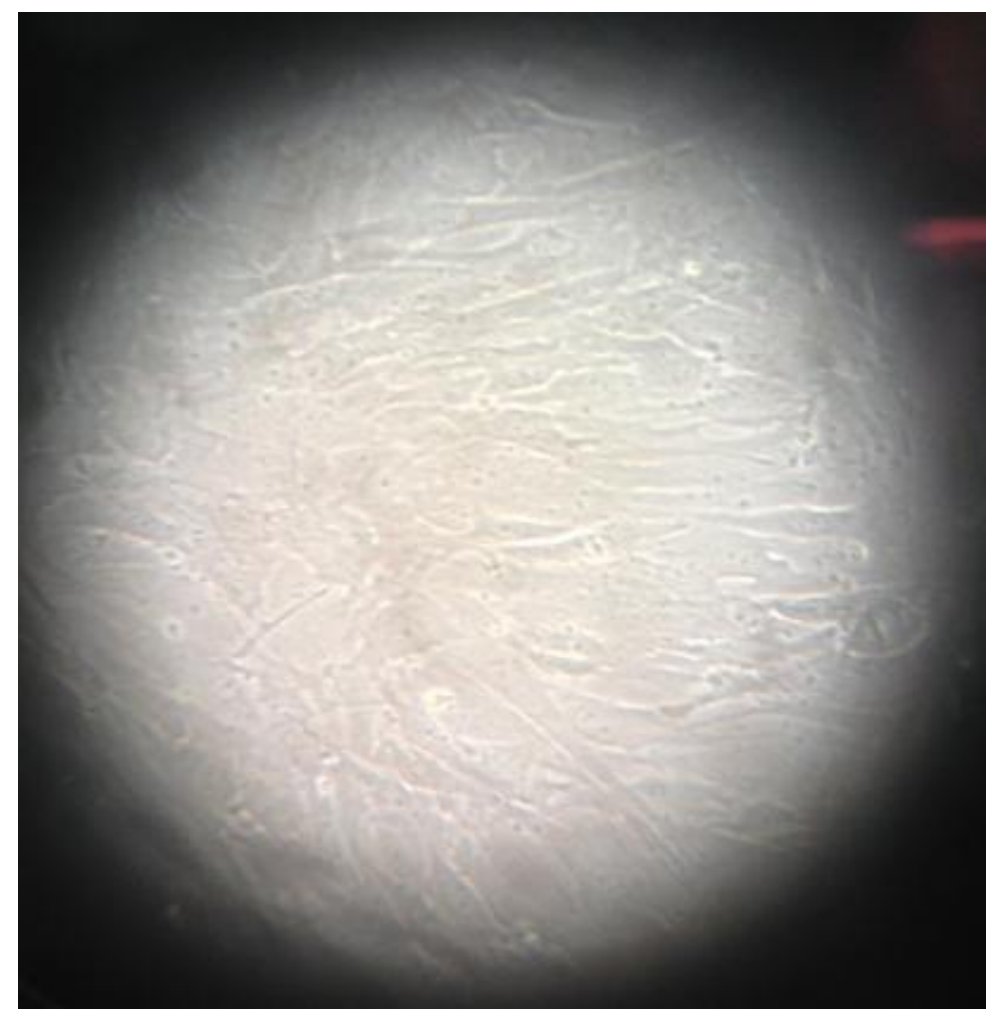

Fig 3.10 Picture of osteoblasts at $\sim 80 \%$ confluency taken under an optical microscope 


\subsubsection{Expansion}

One flask of osteoblasts was expanded to yield a total of 9 flasks of osteoblasts. One extra flask was made for an on-campus senior project and three extra flasks were made to be frozen down for later use. This was achieved by first expanding the original flask into 4 flasks and dedicating one of those to the senior project. The remaining 3 flasks were expanded to 6 flasks and 3 of these flasks were frozen down for later use. The remaining 3 flasks were then expanded into the final 9 flasks. From these 9 flasks, all cells used for deposition on PLGA scaffolds were collected. Four were reserved for compression testing, 3 were reserved for Alizarin Red S staining, and 2 were reserved as extras. This is demonstrated in Figure 3.12.

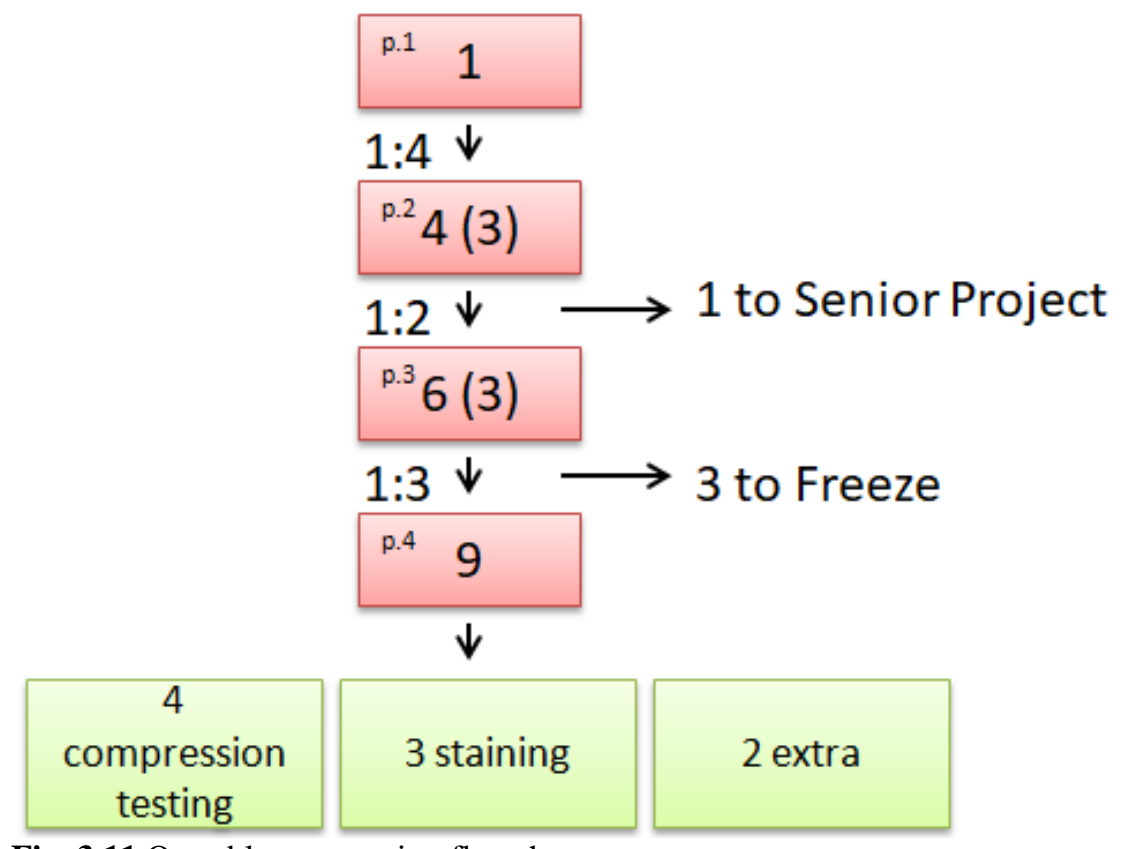

Fig 3.11 Osteoblast expansion flowchart

Each passage of osteoblasts between flasks followed similar steps. The only variability between passages was the ratio to which osteoblasts were passed. Once the flasks in one passage reached a confluency of $80 \%$, they were passed. The necessary amounts of trypsin, trypsin inhibitor, Dulbecco's phosphate-buffered saline (dPBS), and human osteoblast growth medium 
were pipetted into $50 \mathrm{~mL}$ conicals. For each flask being passed, $2 \mathrm{~mL}$ of trypsin, $5 \mathrm{~mL}$ of trypsin inhibitor, $15 \mathrm{~mL}$ of $\mathrm{dPBS}$, and $8 \mathrm{~mL}$ of human osteoblast growth medium were needed. An additional $25 \mathrm{~mL}$ of human osteoblast growth medium were needed for each flask being passed into. These materials were gathered and placed into the water bath for 15 minutes. The necessary number of new flasks were placed inside the hood. Once the 15 minutes were up, the conicals in the water bath were removed, sprayed down with $70 \%$ alcohol and placed upright inside the hood. The first flask of osteoblasts was removed from the incubator and brought inside the hood. One flask of osteoblasts was passed at a time to minimize the osteoblasts' exposure to ambient conditions. The osteoblast growth medium currently in the flask of osteoblasts was aspirated out, and the monolayer of cells was rinsed in $15 \mathrm{~mL}$ of dPBS that was then also aspirated out. Following the rinse, $2 \mathrm{~mL}$ of trypsin were pipetted into the flask, and the flask was rocked back and forth, continuously being checked for cell separation from the bottom of the flask. Once it had been determined that the osteoblasts had sufficiently separated from the bottom of the flask, $5 \mathrm{~mL}$ of trypsin inhibitor and $8 \mathrm{~mL}$ of human osteoblast growth medium were added to the flask. The entire $15 \mathrm{~mL}$ inside the flask was suspended in a pipette and thoroughly mixed before being divided up proportionally between each of the new flasks the osteoblasts were being passed to. For example, if the passage ratio was $3: 1,5 \mathrm{ml}$ of the total $15 \mathrm{ml}$ was added to each of the 3 new flasks. Another $25 \mathrm{~mL}$ of human osteoblast growth medium were then added to each new flask, and the new flasks were placed into the incubator. This process was followed until all osteoblasts had been passed accordingly. 


\subsubsection{Freezing}

Throughout the expansion process, some osteoblasts were frozen down. One flask of cells was frozen down for a senior project, 3 were frozen down for future projects, and the 9 flasks at the end of the expansion process were frozen down until the scaffold fabrication process was finished. A cryovial per flask to be frozen down was obtained and labeled. The first flask to be frozen down was removed from the incubator and brought to the hood. The osteoblast growth medium in the flask was aspirated out, and $15 \mathrm{~mL}$ of dPBS were added to the flask and also aspirated out. Then, $2 \mathrm{~mL}$ of trypsin were added to the flask and rocked back and forth until it was determined that the osteoblasts had properly dislodged from the bottom of the flask. In addition to the $2 \mathrm{~mL}$ of trypsin in the flask, $5 \mathrm{~mL}$ of trypsin inhibitor and $8 \mathrm{~mL}$ of human osteoblast growth medium were added to the flask to neutralize trypsin's effects. The resultant cell suspension was collected into a conical. The conical was then placed in a centrifuge and balanced by a conical with $15 \mathrm{~mL}$ of water. The conical was centrifuged in an International Equipment Company model CL CLINICAL centrifuge for 4 minutes at 2,000rpm while freezing medium was made. Freezing medium is $80 \%$ human osteoblast growth medium, $10 \%$ dimethyl sulfoxide (DMSO), and 10\% fetal bovine serum (FBS). $1 \mathrm{~mL}$ is needed per cryovial. The necessary constituents of the freezing medium were micropipetted together in the hood and vortexed thoroughly. The centrifuged conical of cells was then sprayed with $70 \%$ IPA and brought inside the hood as well. The medium in the conical was aspirated off leaving behind a pellet of osteoblasts. The pellet was resuspended in $1 \mathrm{ml}$ of freezing medium and mixed gently with a pipette to break up any clumps of osteoblasts. The freezing medium with cells was then pipetted into a labeled cryovial and placed inside a ThermoScientific Mr. Frosty freezing 
container. Once all cryovials had been made, Mr. Frosty was placed in a $-80^{\circ} \mathrm{C}$ freezer for 24 hours and then transferred into a liquid nitrogen Dewar for long term storage.

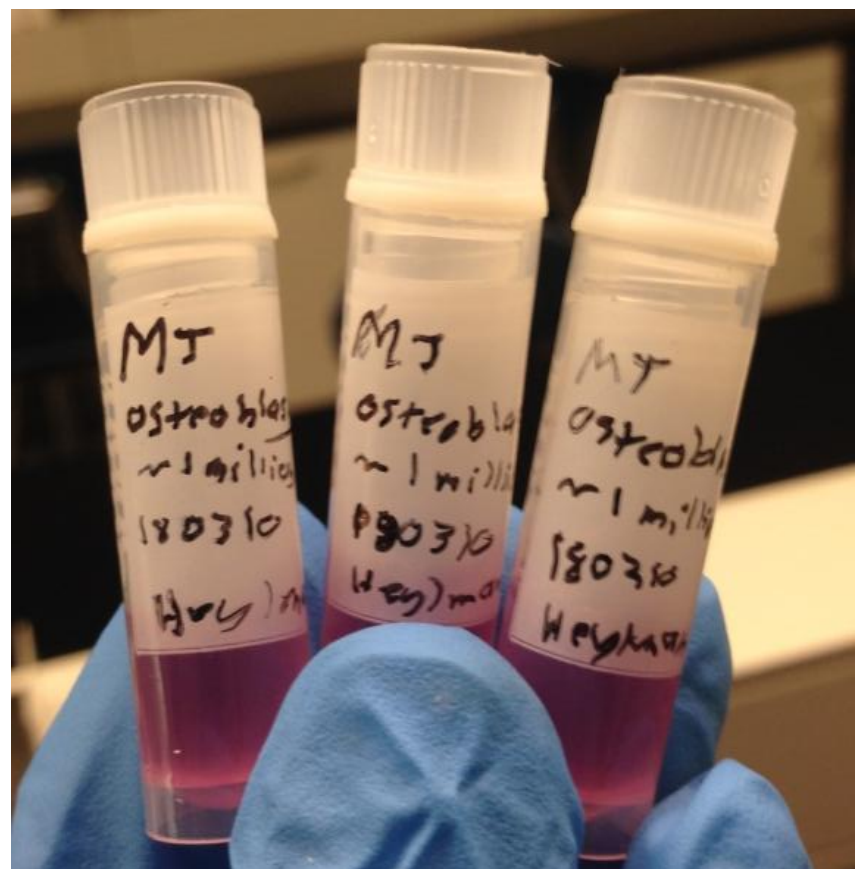

Fig 3.12 Three cryovials of frozen human adult osteoblasts

\subsubsection{Counting}

There were three instances along the cell expansion process where the number of osteoblasts present in a flask were counted: before freezing down osteoblasts from passage 3, before freezing down the osteoblasts from passage 4, and before depositing osteoblasts onto the PLGA scaffolds. The cell counts were performed the same way during each of these instances. After a flask of cells had been trypsinized off the surface of a flask and stored in a conical with a certain volume of medium (specifics are given in their respective sections), a micropipette was used to collect $100 \mu \mathrm{L}$ of the cell suspension. The $100 \mu \mathrm{L}$ of cell suspension was added to a $2 \mathrm{~mL}$ collection tube. Outside of the biological safety cabinet, $100 \mu \mathrm{L}$ of Trypan Blue was pipetted into the same collection tube and mixed within the micropipette tip. The $200 \mu \mathrm{L}$ of Trypan Bluestained osteoblast suspension was pipetted into a BRAND hemocytometer. The hemocytometer 
was placed under an optical microscope and live and dead cells were counted. The live cells were transparent and the dead cells were stained blue by the Trypan Blue. Once an average amount of viable cells per volume was determined, reverse calculations were performed to estimate how many viable cells were in the original flask or flasks of cells.

\subsubsection{Growth of Osteoblasts on Scaffolds}

Once the scaffolds had all been fabricated, 8 of the 9 vials of frozen osteoblasts were plated again following the same steps as the original plating procedure. When it was determined the 8 flasks of osteoblasts were one day away from reaching $80 \%$ confluency a 48 -well plate with 25 scaffolds in it was set up for cell deposition. Four rows of the 48 -well plate were to be used: two with Cell Applications, Inc. Human Osteoblast Differentiation Medium (ODM), one with Dulbecco's Phosphate Buffered Saline (dPBS), and one without solution. The 25 PLGA scaffolds were first soaked in $70 \%$ ethanol inside of the biological safety cabinet for 10 minutes for sterility. Then, they were removed from the ethanol and washed in $\mathrm{dPBS}$, which is sterile, 5 times each to rid them of any excess ethanol which could later cause damage to deposited osteoblasts. The sterilized scaffolds were then placed in the 48 -well plate with 7 in the rows A,B, and $\mathrm{C}$ and 4 in row $\mathrm{D}$. One $\mathrm{mL}$ of ODM was pipetted into each well with a scaffold in rows $\mathrm{A}$ and B. One mL of dPBS pipetted into each well with a scaffold in row C. Row D was left without solution in it. Figure 3.14 illustrates this 48 -well plate setup. The 48 -well plate was then placed in the incubator for 24 hours to allow the scaffolds to soak in their respective solutions. 


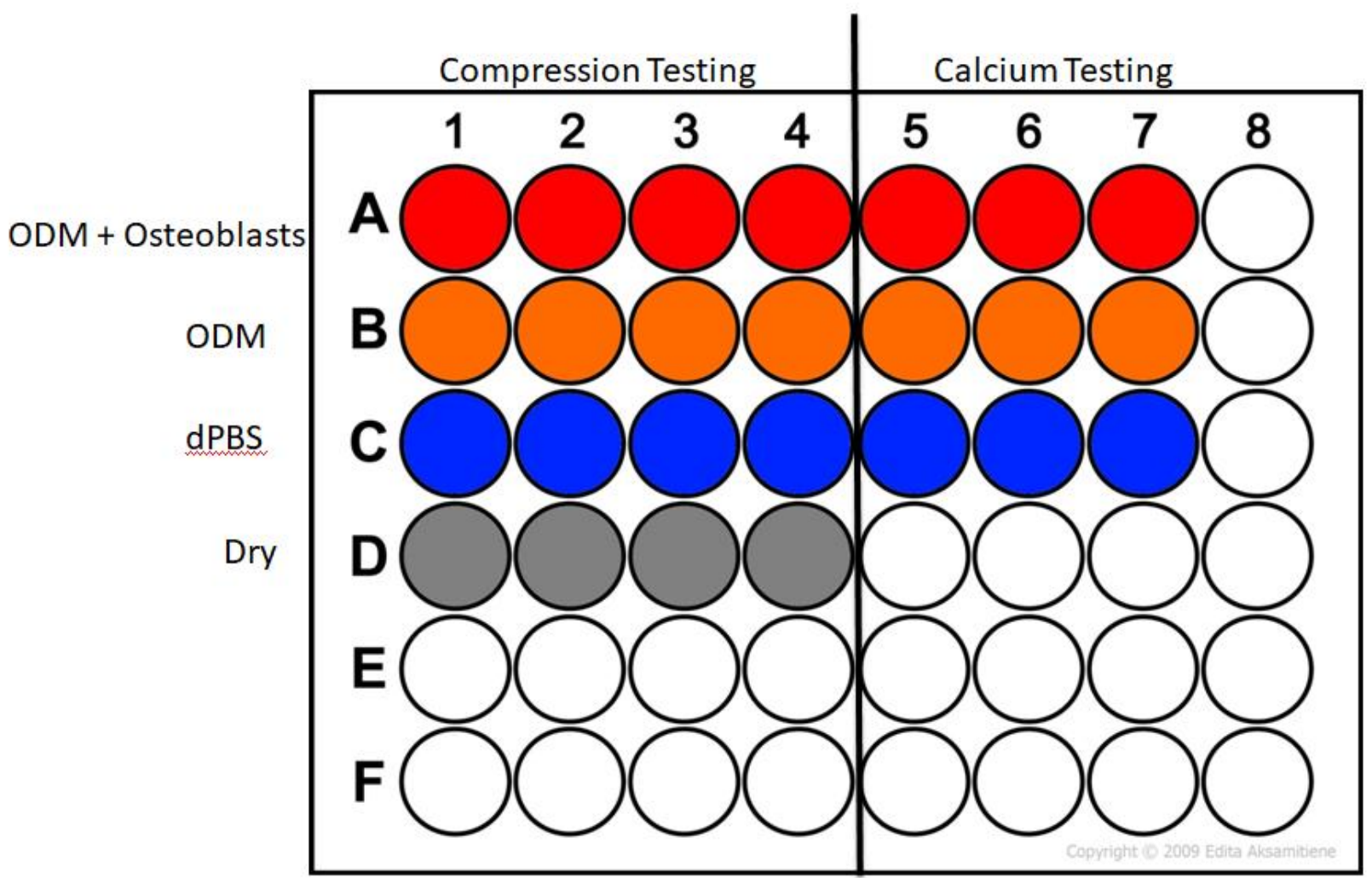

Fig 3.13 48-well plate setup for scaffold soak and osteoblast growth with the respective media in each well listed on the left and the dedicated experiment for each well listed above

On the second to last day of cell proliferation in the 8 plated flasks, 4 of the flasks had been contaminated with a fungal infection likely caused by a medium spill in the incubator. Immediately, these 4 flasks were properly disposed of and the incubator thoroughly cleaned. The experiment was continued on with the remaining 4 flasks of osteoblasts in hope that there would still be enough cells to accomplish the goal. When the 4 remaining plated flasks of osteoblasts reached $80 \%$ confluency, the osteoblasts were ready to be deposited onto the scaffolds. The medium was aspirated out from one flask of osteoblasts. The osteoblasts were rinsed in dPBS that was also aspirated out. Then, $2 \mathrm{~mL}$ of trypsin were added to the flask and rocked back and forth until it had been determined that the osteoblasts had sufficiently been stripped from the surface of the flask. Afterwards, $8 \mathrm{~mL}$ of ODM was added to the flask. The entire $10 \mathrm{~mL}$ inside the flask was then pipetted into a 50mL conical. This was repeated for each flask of osteoblasts, 
resulting in one $50 \mathrm{~mL}$ conical with $40 \mathrm{~mL}$ of osteoblasts, neutralized trypsin, and ODM. One hundred $\mu \mathrm{L}$ of this solution was pipetted with a micropipette into a $2 \mathrm{~mL}$ collection tube for cell counting. Upon completing the cell count detailed in Section 3.2.4, it was discovered that there were 8.55 million osteoblasts in the $40 \mathrm{~mL}$ inside the $50 \mathrm{~mL}$ conical which was greater than 7 million osteoblasts required for the experiment. Another $50 \mathrm{~mL}$ conical was filled with $39.9 \mathrm{~mL}$ of water. These two conicals were placed opposite of each other into an International Equipment Company model CL CLINICAL centrifuge and spun on setting 4 (2,000rpm) for 4 minutes. After centrifuging, the conical with osteoblasts was sterilized with $70 \%$ IPA and brought back into the biological safety cabinet. The supernatant was removed from the conical, and the osteoblasts were mixed inside of a pipette and pipetted into a conical with $1.7 \mathrm{~mL}$ of ODM, the necessary amount to have approximately 1 million cells in every $0.2 \mathrm{ml}$ of ODM.

The 48-well plate was then removed from the incubator for osteoblast deposition. The medium and dPBS within each well of the 48-well plate was aspirated out carefully making sure not to touch the scaffolds with the aspirating pipette. Then, $0.2 \mathrm{~mL}$ of the osteoblast solution was pipetted directly onto the 7 scaffolds in row A. After each deposition the remaining solution that seeped out of the scaffold was pipetted back onto the scaffold 5 times to ensure as many osteoblasts stuck to the scaffold as possible. The 48-well plate was then placed back inside the incubator for 2 hours to allow the osteoblasts to lodge within the scaffolds before the remaining medium was added. After 2 hours, the 48-well plate was retrieved from the incubator and brought back inside the biological safety cabinet. The remaining $0.8 \mathrm{~mL}$ of ODM was added to each scaffold-containing well in Row A, 1mL of ODM was added to each scaffold-containing well in Row B, and $1 \mathrm{~mL}$ of dPBS was added to each scaffold-containing well in Row C. The 48well plate was then placed back in the incubator and the dPBS and medium were changed every 
other day for what was originally planned to be 10 days, but on day 6 , it was found that the contamination had made its way to the 48 -well plate. The scaffolds were removed from the plate and compression tests were performed that day. The 9 scaffolds used in the calcification tests were also frozen in OCT gel, detailed in Section 3.4, that day.

\subsection{Compression Testing}

Compression tests were performed on 16 scaffolds to determine the Young's modulus of each. Four scaffolds were soaked in ODM with osteoblasts, 4 were soaked in ODM without osteoblasts, 4 were soaked in dPBS, and 4 were left dry. The hope was to find that the presence of osteoblasts increased the Young's modulus, and therefore material stiffness, in the PLGA scaffolds. The scaffolds soaked in ODM without osteoblasts were the control and main comparison. The scaffolds soaked in dPBS were to determine if the ODM was contributing to any observed difference in Young's moduli from dPBS. The dry scaffolds were to determine how much of an impact being wet instead of dry had on the Young's moduli of the scaffolds.

An Instron 3342 single-column tensile/compression testing machine was used with the included software, Bluehill 3, to measure the Young's moduli of scaffolds in compression. Compression platens were designed to be used with the Instron by Leah Torres. The platens were $50 \mathrm{~mm}$ in diameter and have a capacity of $10 \mathrm{kN}$. The schematic for the platens was then sent to the Cal Poly machine shop to be fabricated from 316 stainless steel. A 500N load cell was used with the Inston, meaning the compessive load was not to exceed $500 \mathrm{~N}$ during testing. The setup of the Instron with compression platens can be seen in Figure 3.15. A program was setup within the Bluehill 3 software to run the compression tests. The program was set to measure the maximum load, Young's modulus, and ultimate compressive yield of each scaffold, and export 
the raw data of load over extension into a .csv file. The program was also set with a constraint to stop testing when the load reaches $450 \mathrm{~N}$ to protect the load cell. There were also emergency stops for if the two platens got too close together. A starting position for the top platen was arbitrarily picked to be the 6.6in. tick mark on the vertical ruler on the Instron.

The scaffolds were removed from the 48-well plate with tweezers and placed on labeled paper towels to dry for 10 minutes. One dry scaffold was placed in the center of the bottom platen. In Bluehill 3, the load and the extension were zeroed. This is the only time the extension was zeroed, however the load was zeroed at the beginning of each compression. The program was set to run, and a plot for load $(\mathrm{N})$ over extension $(\mathrm{mm})$ was plotted and the Young's modulus was automatically calculated. The Bluehill 3 program exported the raw data of load over extension along with a spreadsheet with the values of maximum load $(\mathrm{N})$, the load at ultimate compressive yield (N), and the Young's modulus (MPa). The four dry scaffolds were all run and plotted onto one graph, then the dPBS-soaked scaffolds, then the medium-soaked scaffolds, then the medium-soaked scaffolds with osteoblasts. The platens were removed from the Instron and cleaned with $70 \%$ IPA in between each run as drops of dPBS and medium could get left behind between each compression. The compression platens were autoclaved once all compression tests were run. 


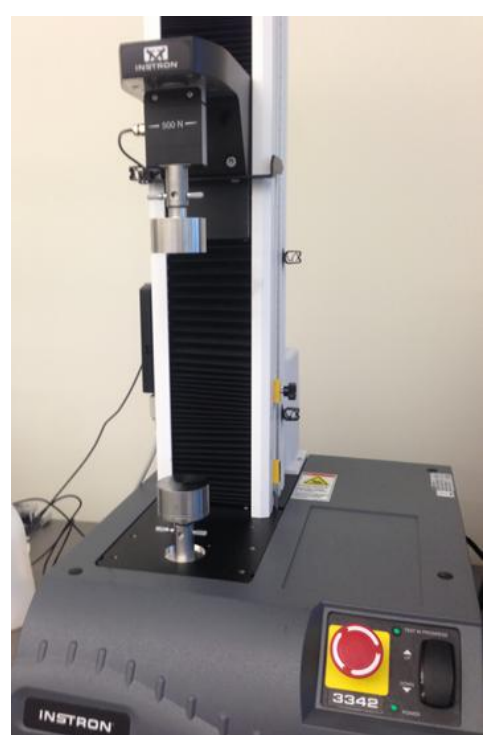

Fig 3.14 Instron 3342 single-column tensile/compression testing machine with top and bottom compression platens attached

\subsection{Staining}

On the same day that the compression tests were run, the 9 remaining scaffolds were prepared for cryosectioning. The scaffolds were washed in $1 \mathrm{~mL}$ dPBS twice within their wells. They were then left to sit in $1 \mathrm{~mL}$ of $70 \%$ ethanol within their wells for 10 minutes before the ethanol was also aspirated out. Each scaffold was placed at the bottom of a labeled cryosectioning mold top down that was then filled halfway with optimum cutting temperature gel (OCT gel). The molds with OCT gel were then dipped in a portable Dewar filled with liquid nitrogen via tweezers until all the OCT gel was frozen as seen if Figure 3.16a. These molds were stored in a $-18^{\circ} \mathrm{C}$ freezer for 48 hours.

After 48 hours, one mold was cut with a razor and popped open. The frozen OCT gel block was removed and placed on the center of a die with OCT gel on the middle. The die was placed on a shelf in a ThermoScientific Cryotome FSE cryostat to allow the fresh OCT gel to harden. The die was then placed in the main apparatus of the cryostat, and the cryostat blade was placed in its proper position as seen in Figure 3.16b. The cryostat was set to take $60 \mu \mathrm{m}$ sections 
(cryostat sections are generally between $40 \mu \mathrm{m}$ and $60 \mu \mathrm{m}$, however the $40 \mu \mathrm{m}$ setting failed to produce full scaffold slices). The blade was moved up against the surface of the frozen OCT block and the handle on the outside of the cryostat was spun to start taking sections of the OCT block. Sections that were deemed to be of proper quality were picked up with a tweezer and placed onto a labeled Vectabond-cured optical microscope slide. A quality section entailed a full section that included an entire cross-section of the scaffold. Efforts were made to only collect slices that did not roll up, however this was shown to be quite a difficult task. Five quality sections from each scaffold were placed on a slide dedicated to that scaffold as seen in Figure 3.16c. One dPBS-soaked scaffold OCT block was discarded due to a failure to retrieve any quality cryosections. This resulted in 8 slides being created: 2 with 5 slices of dPBS-soaked scaffold, 3 with 5 slices of ODM-soaked scaffold, and 3 with 5 slices of ODM-soaked scaffolds with deposited osteoblasts.

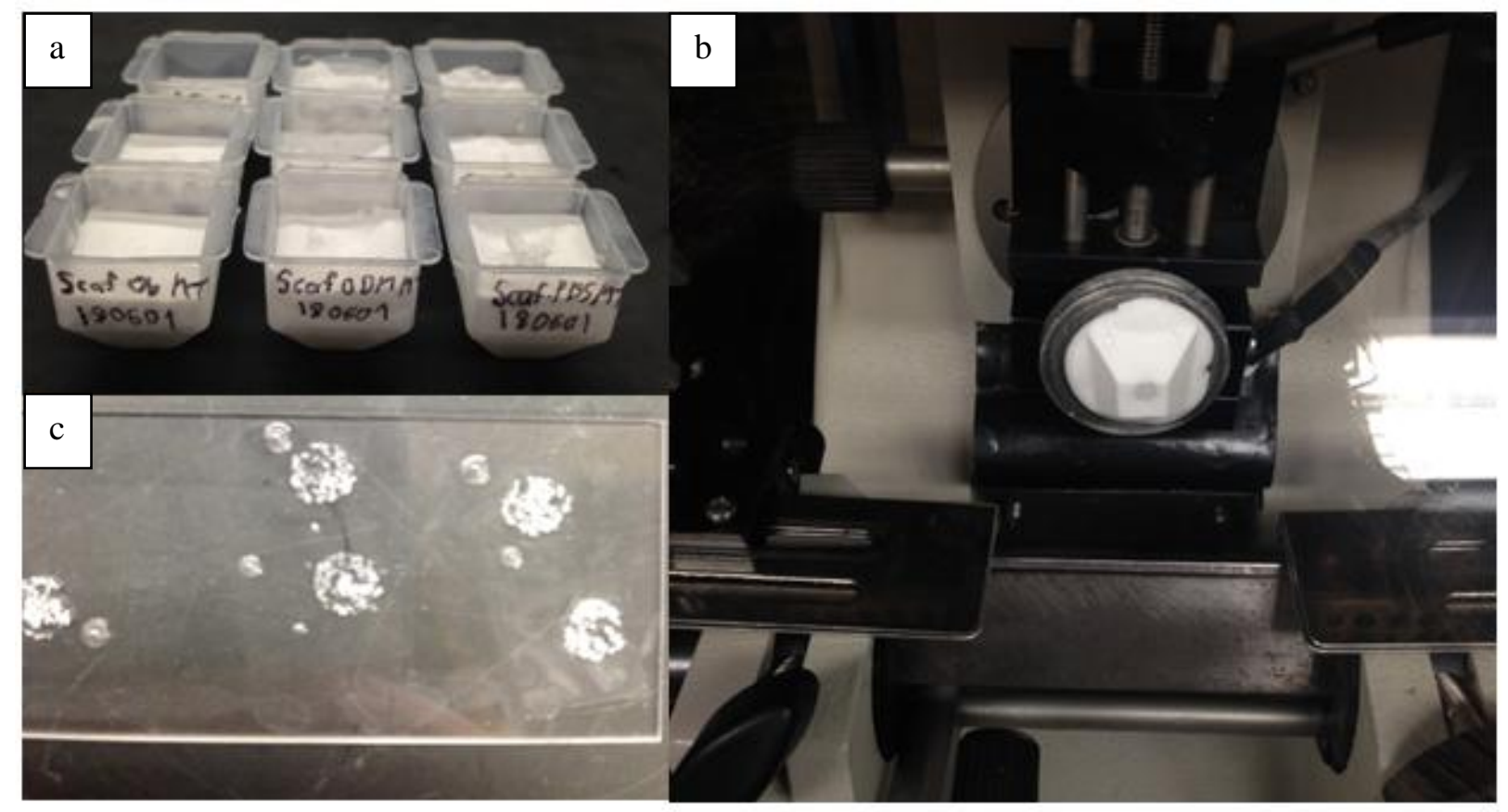

Fig 3.15 a) Scaffolds frozen in OCT gel in molds, b) Frozen OCT gel block in cryostat on die, c) Five cryosections from a dPBS-soaked scaffold on a Vectabond-treated slide 
The next step was to stain and preserve the scaffold cryosections. Alizarin Red S purchased from Sigma Aldrich (Model \# MFCD00013049) was used to stain for calcium present in the scaffold cryosections. A $75 \mathrm{~mm} \times 25 \mathrm{~mm}$ glass staining jar was filled with $200 \mathrm{~mL}$ of deionized water, and 200mg of Alizarin Red S dye was added to the water and stirred with a stirring rod under a fume hood. The 8 slides of cryosections were placed in a staining tray and submerged in the 1\% Alizarin Red S solution for 4 minutes as seen in Figure 3.17a. The tray was pulled out of the solution and excess staining was shaken off as best as possible. The slides were placed on a paper towel and left to dry for 20 minutes as seen in Figure 3.17b. The Alizarin Red S solution was disposed of in an organic solvents waste container.

Images of the stained slides were taken with an Olympus CKX41 inverted microscope with the software QCapture Pro 6. All images were taken at 4x magnification, and 5 images were taken of each slide of the spots with most Alizarin Red S staining showing. It was noticed that there was physical Alizarin Red S dye on the slides that showed up in the images without staining anything in particular, so we decided to take more pictures after going through the steps to preserve the slides.

To preserve the slides, the slides were dipped in staining jars filled with $200 \mathrm{~mL}$ of pure acetone, $200 \mathrm{~mL}$ of a 1:1 solution of acetone and xylene, and then $200 \mathrm{~mL}$ of pure xylene. The slides were dipped for one second 20 times in each solution and drip dried as well. The xylene and acetone-xylene solution were disposed of in a xylene waste container, and the acetone was disposed of in a organic solvents waste container.

Pictures were taken again with the inverted optical microscope after undergoing the steps to preserve the slides. These pictures were determined to show a more accurate representation of what was actually being stained and what was excess Alizarin Red S dye. 


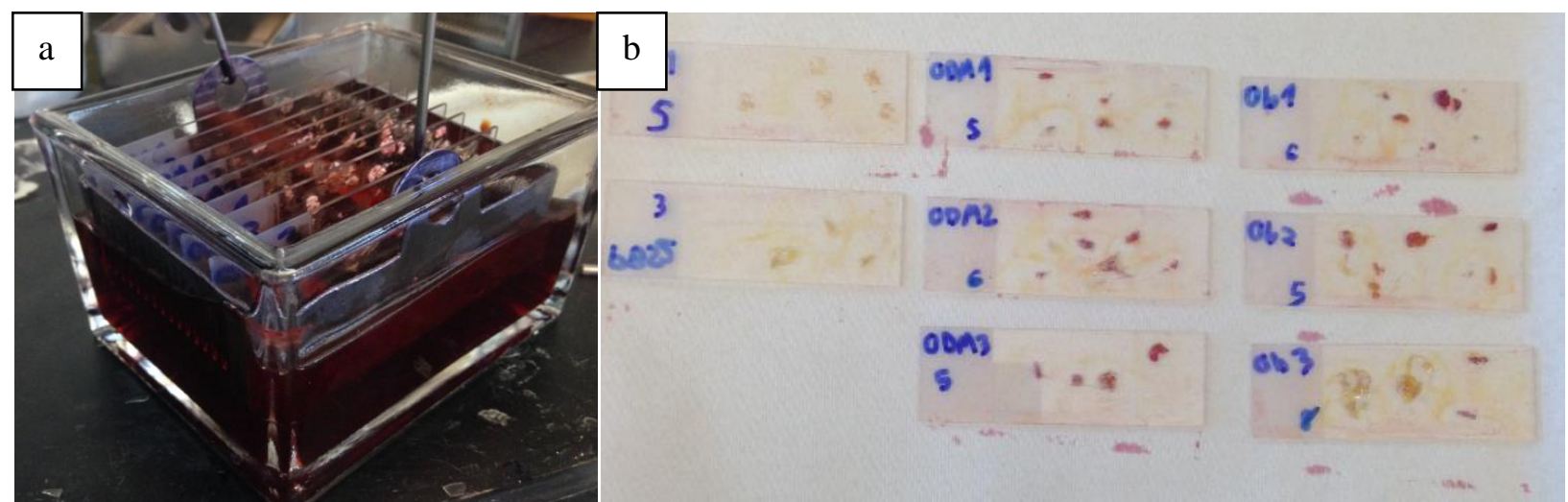

Fig 3.16 a) Cryosections being pulled out of Alizarin Red S solution, b) Slides after Alizarin Red S staining

Along with the qualitative analyses made on these images, Fiji ImageJ was used to make further quantitative analyses. The settings for color threshold were set as shown in Figure 3.18. These settings were determined since they best highlighted aggregated red clumps of Alizarin Red S-stained material as shown in Figure 3.19, where a\&d show the original images, b\&e show the aggregated clumps filtered via the white mask, and c\&f show that filtered mask selected for measurement. These same settings were used for each image and the filtered pixels were measured and these values were recorded into a Microsoft Excel spreadsheet.

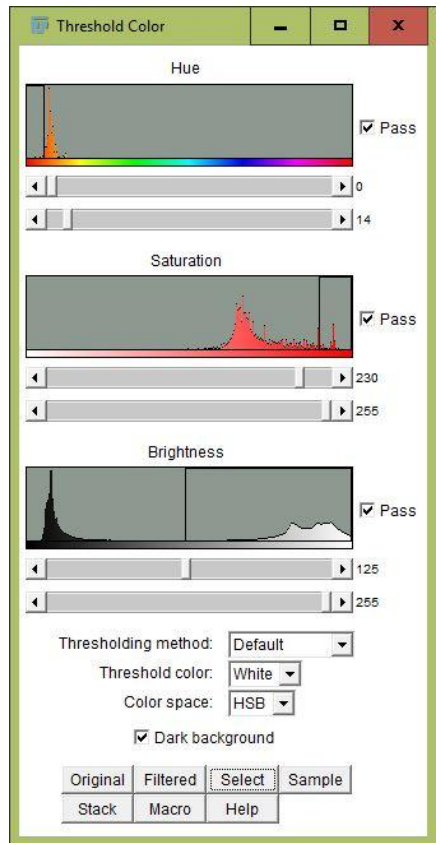

Fig 3.17 Threshold Color settings in Fiji ImageJ for detecting Alizarin Red S-stained material 


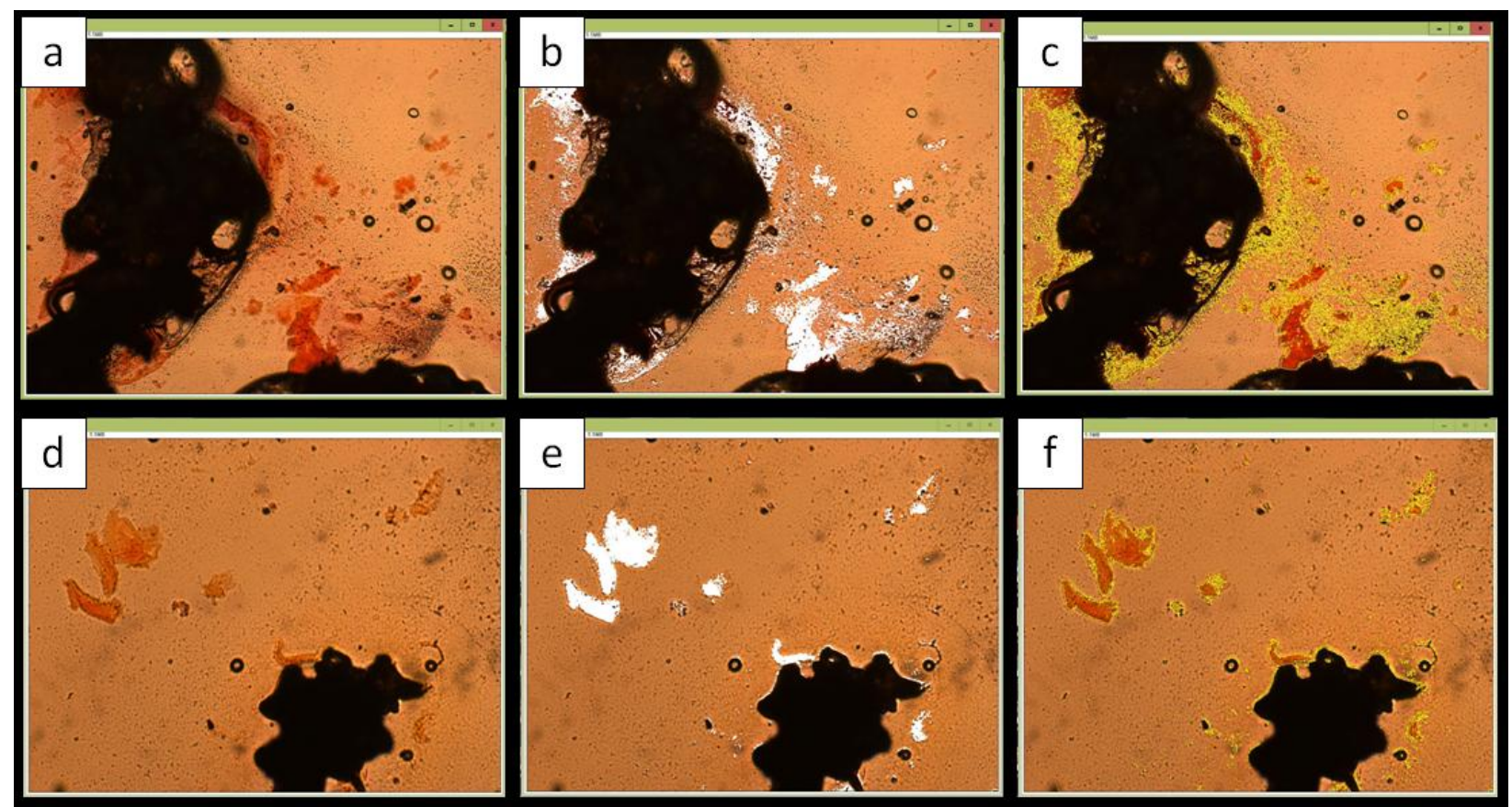

Fig 3.18 a\&d) Original inverted optical microscope images of Alizarin Red S-stained cryosections, b\&e) Filtered images of Alizarin Red S-stained cryosections, $\mathbf{c \& f}$ ) Filtered regions selected for measurements 


\section{Chapter 4}

\section{RESULTS AND DISCUSSION}

\subsection{Comparison of Gas Foaming Release Methods}

We originally planned to use a proportional-integral-derivative controller (PID) to control the release of carbon dioxide gas pressure from the gas foaming chamber before switching to sending a constant $2 \mathrm{~V}$ to the positioner to open the release valve. This section shows the comparison of the two methods of release both graphically and via scanning electron microscope (SEM) images of scaffolds produced from both methods.

Figures 4.1 and 4.2 show six consecutive pressure over time graphs for the two different gas foaming release methods. Tables 4.1 and 4.2 collect the line of best fit constants for the second-degree polynomial lines of best fit from each graph in Figures 4.1 and 4.2 and then calculate the coefficient of variation $(\mathrm{CoV})$ between the constants for each gas foaming method. Second-degree polynomial fits were chosen since they provided the best fits, $\mathrm{R}^{2}$ values closest to 1, overall between different sets of data. The percent difference between the CoVs of each line of best fit constant between each gas foaming method tells the percent difference in variability between the different lines of best fit for the two gas foaming methods. Table 4.3 shows that the lines of best fit for the pressure releases performed with the $2 \mathrm{~V}$ method are $59.43 \%$ less variable in the $\mathrm{x}^{2}$ term, $50.98 \%$ less variable in the $\mathrm{x}$ term, and $32.76 \%$ less variable in the constant term than the pressure releases performed via the PID. This allowed us to claim that the constant $2 \mathrm{~V}$ method produces more consistent, reproducible gas foaming pressure releases than the PID method. 

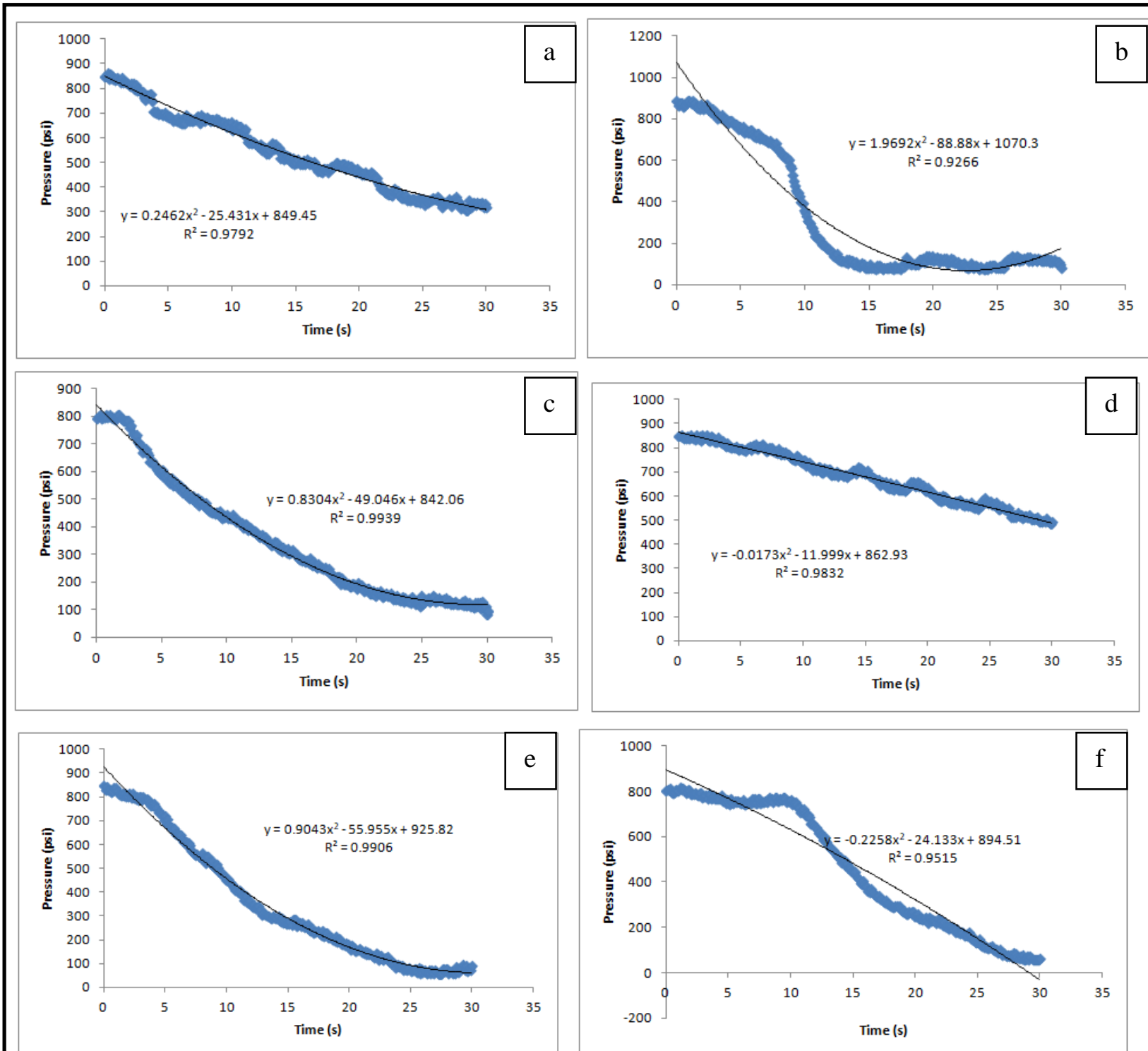

Fig 4.1 Pressure vs. time graphs of six consecutive gas releases utilizing the PID to control carbon dioxide gas release

Table 4.1 Line of best fit constants from Fig 4.1 and the coefficient of variation $(\mathrm{CoV})$ for each constant between gas foaming releases

\begin{tabular}{|c|c|c|c|c|c|c|c|c|}
\hline & \multirow{2}{*}{\multicolumn{6}{|c|}{ Graph }} & \multirow[b]{3}{*}{$\mathrm{CoV}$} \\
\hline & & & & & & & & \\
\hline & & a & b & c & d & e & $f$ & \\
\hline \multirow{3}{*}{ Lobf Constant } & $x^{\wedge} 2$ & 0.25 & 1.97 & 0.83 & -0.02 & 0.90 & -0.23 & 130.03 \\
\hline & $\mathbf{x}$ & -25.43 & -88.88 & -49.05 & -12.00 & -55.96 & -24.13 & -65.89 \\
\hline & c & 849.45 & 1070.30 & 842.06 & 862.93 & 925.82 & 848.51 & 9.89 \\
\hline
\end{tabular}



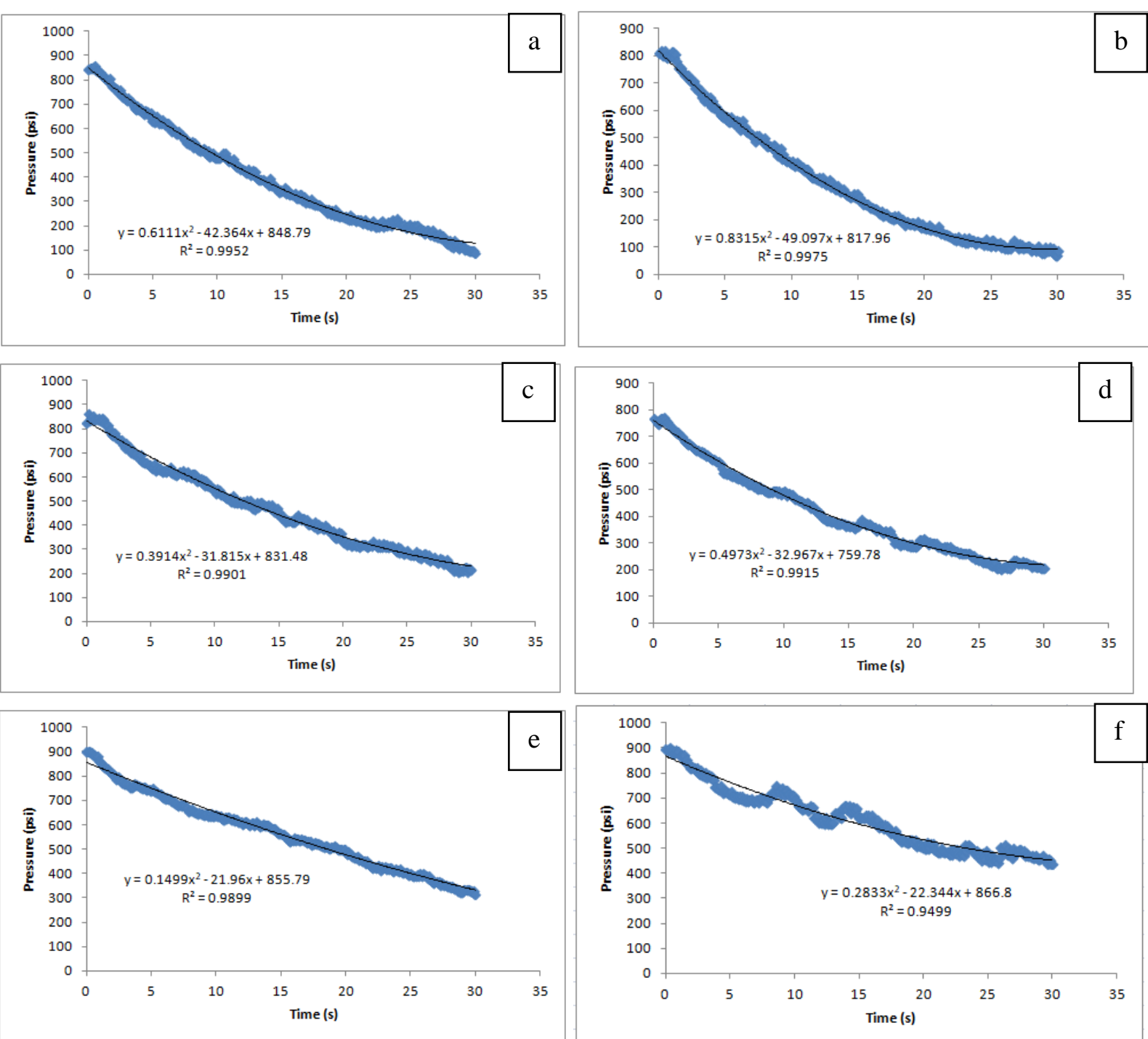

Fig 4.2 Pressure vs. Time graphs of six consecutive gas releases where a constant $2 \mathrm{~V}$ was sent to the positioner

Table 4.2 Line of best fit constants from Fig 4.2 and the coefficient of variation (CoV) for each constant between gas foaming releases

\begin{tabular}{|c|c|c|c|c|c|c|c|c|}
\hline & \multicolumn{6}{|c|}{ Graph } & \multirow[b]{2}{*}{$\mathrm{CoV}$} \\
\hline & & a & b & c & d & e & $f$ & \\
\hline \multirow{3}{*}{ Lobf Constant } & $\mathrm{x}^{\wedge} 2$ & 0.61 & 0.83 & 0.39 & 0.50 & 0.15 & 0.28 & 52.75 \\
\hline & $\mathbf{x}$ & -42.36 & -49.10 & -31.82 & -32.97 & -21.96 & -22.34 & -32.30 \\
\hline & c & 848.79 & 817.96 & 831.48 & 759.78 & 855.79 & 866.80 & 4.65 \\
\hline
\end{tabular}

Table 4.3 Calculated percent difference between the coefficients of variations (CoV), a measure of difference in variability, in the lines of best fit constants for the two gas foaming methods.

\begin{tabular}{|c|r|c|c|c|}
\cline { 3 - 5 } \multicolumn{2}{c|}{} & CoV PID & CoV 2V & \% less variable \\
\hline \multirow{3}{*}{ Lobf Constant } & $\mathbf{\mathbf { x } ^ { \wedge } 2}$ & 130.03 & 52.75 & 59.43 \\
\cline { 2 - 5 } & $\mathbf{x}$ & -65.89 & -32.3 & 50.98 \\
\cline { 2 - 5 } & $\mathbf{c}$ & 9.89 & 6.65 & 32.76 \\
\hline
\end{tabular}


The next comparison we wanted to make was between SEM images of scaffolds made with the two different methods. We originally intended to compare the number of pores in a frame and the size of the maximum diameters of these pores, but upon viewing the scaffolds under the SEM it was determined impractical to establish what was considered a pore and where to start the measurement of maximum diameter sizes from. However, even though these results are not fully quantitative, there is a lot to be compared between the impacts that the two gas foaming release methods had on scaffold formation, and measurements were made in ImageJ to get a better representation of the difference in porosity. Some measurements within the SEM program of what were considered to be pores were made as well to put perspective to the images. The gas foaming pressure release graph for the scaffolds made utilizing the PID that are being analyzed in these SEM images can be viewed in Figure 4.1f. The gas foaming pressure release graph for the scaffolds made via sending a constant $2 \mathrm{~V}$ to the positioner that are being analyzed in these SEM images can be viewed in Figure 4.2f.

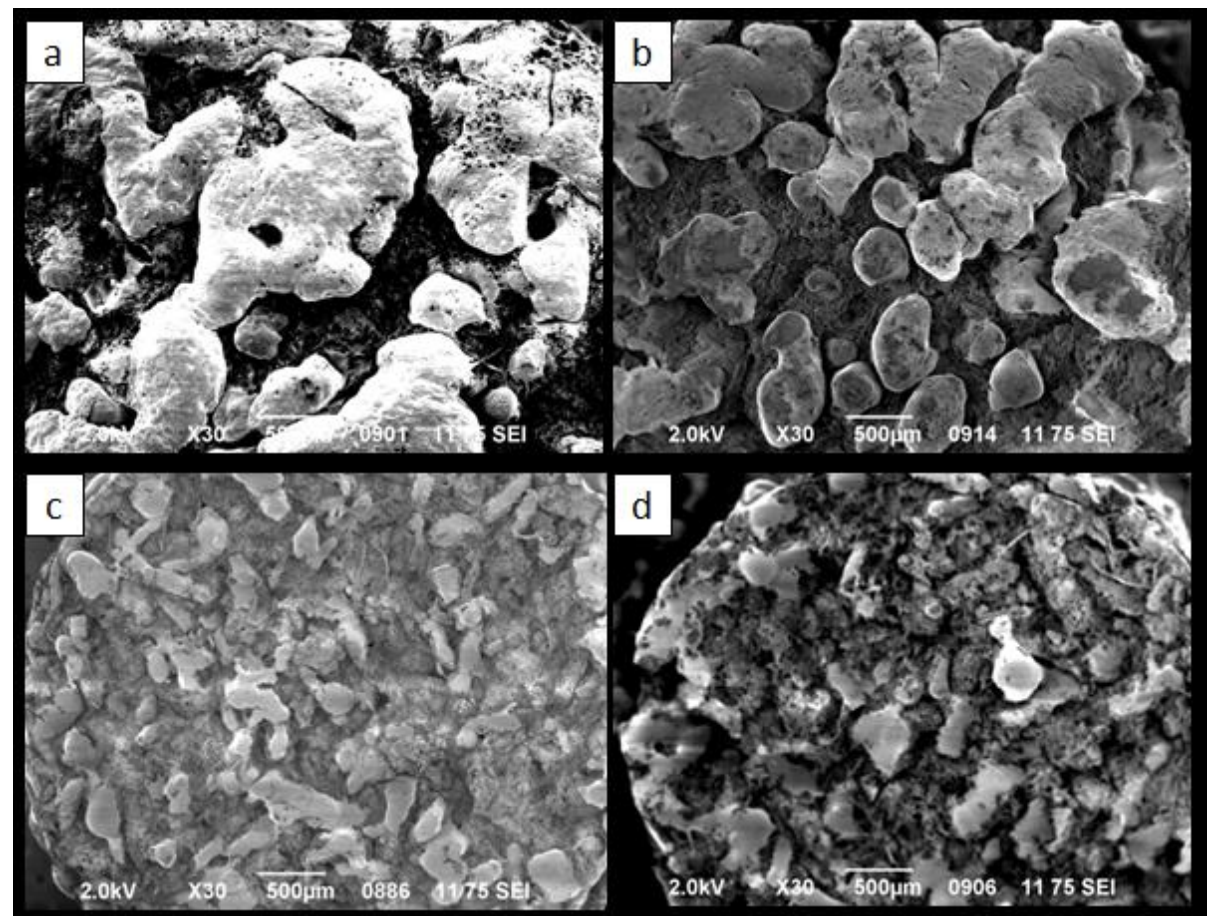

Fig 4.3 SEM images taken at 30x magnification of scaffolds made by (a\&b) using a PID (c\&d) sending a constant $2 \mathrm{~V}$ to the positioner 
Figure 4.3 shows SEM images of the scaffolds made via the two different methods at 30x magnification to capture their major surface structures. It can be seen that the scaffolds made utilizing the PID have large, smooth, puffed-out features likely caused by too rapid of a gas foaming release, resulting in an over-nucleation of gas pores in the PLGA particles. Looking at the scaffolds made by sending a constant $2 \mathrm{~V}$ to the positioner at this magnification, the presence of pores is not clearly evident. There appears to be a fibrous layer covering the entire top layer of the scaffold. This is likely due to the gold that was sputtered on to the scaffolds to prevent them from overcharging in the SEM. However, when looking at SEM images at 100x magnification the approximate location of pores can be determined with higher accuracy.

Figures 4.4 and 4.5 show SEM images of the scaffolds made via the two different methods at 100x magnification to analyze the size and distribution of pores on the surface of these scaffolds. Osteoblasts are approximately $15-20 \mu \mathrm{m}$ in diameter (Tanaka-Kamioka et al., 1998) and pits between $30-40 \mu \mathrm{m}$ in diameter on scaffold surfaces have been shown to best promote osteogenic activity in the presence of bone-forming cells (Halai et al., 2014). Pores larger than the $30-40 \mu \mathrm{m}$ diameter range are still desirable as there will be smaller pits within these larger pores that do fall within this range. Therefore, scaffolds with pores at least 30um in diameter would be suitable for osteoblast attachment, growth, and calcium deposition onto the scaffold. The SEM images in Figure 4.4 demonstrate a lack of evenly-distributed pores that are at least $30-40 \mu \mathrm{m}$ in diameter at the surface of the scaffolds made with the PID. There are a few pores that fit these parameters most prominently localized where the smooth puffed-out PLGA surfaces interface with the more fibrous layers underneath. However, it is doubtful that these pores penetrate far enough into the PLGA matrix to create an interconnected system of pores. 


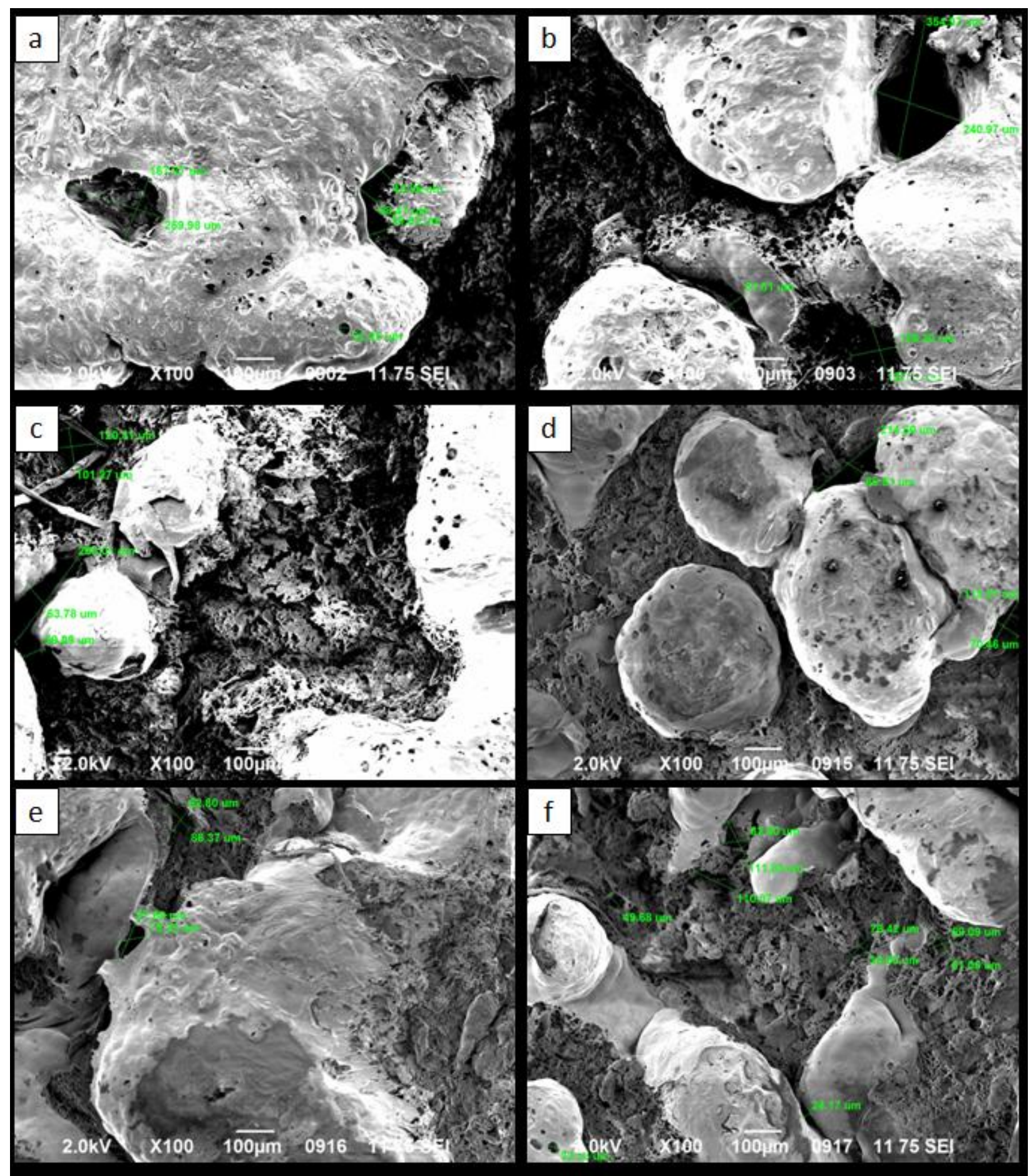

Fig 4.4 SEM images taken at 100x magnification for scaffolds made utilizing the PID. Images (a-c) are of the scaffold shown in Figure 4.3(a). Images (d-f) are of the scaffold shown in Figure 4.3(b). 

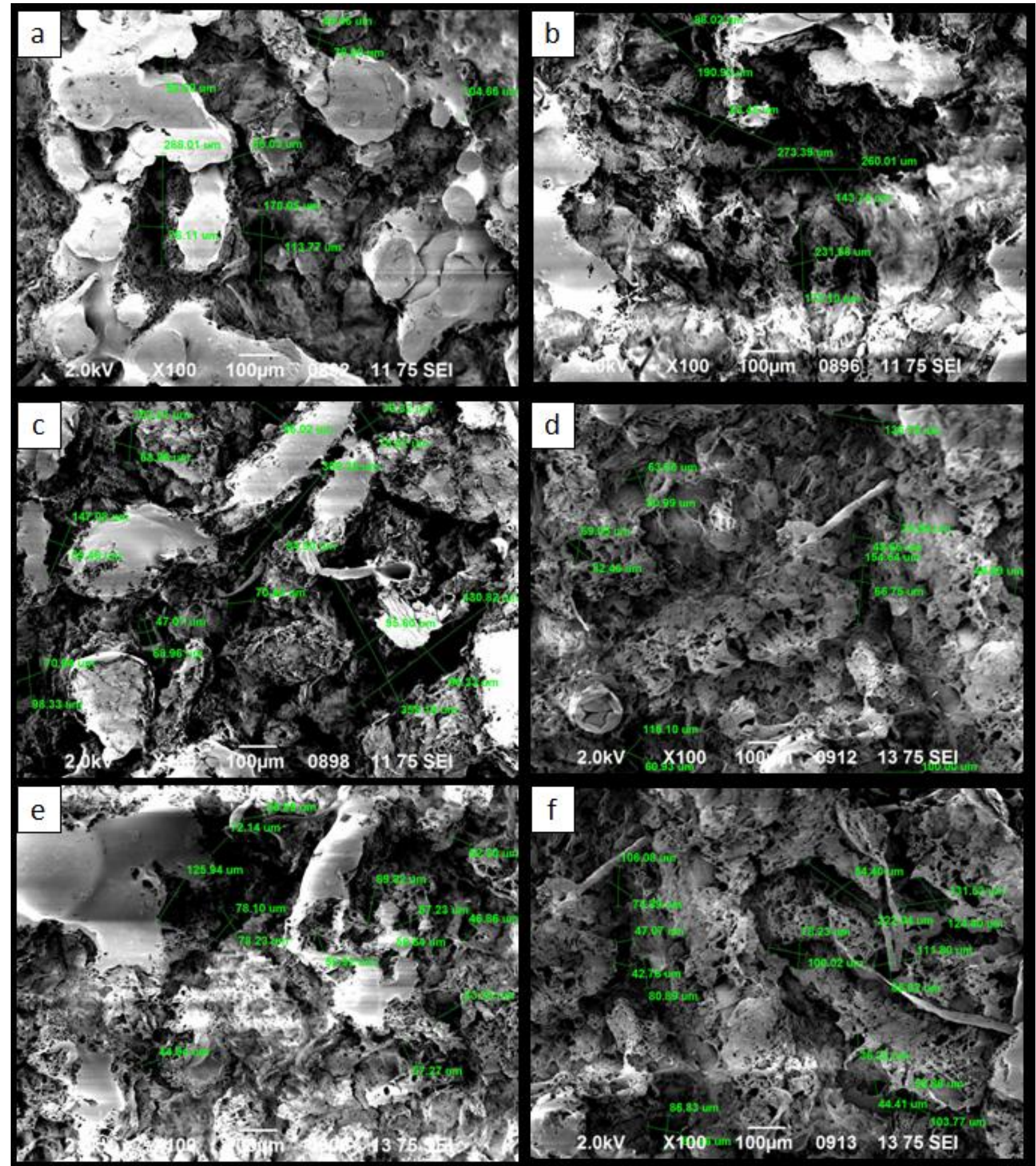

Fig 4.5 SEM images taken at 100x magnification for scaffolds made by sending a constant $2 \mathrm{~V}$ to the positioner. Images (a-c) are of the scaffold shown in Figure 4.3(c). Images (d-f) are of the scaffold shown in Figure 4.3(d). 
The 100x magnification SEM images of the scaffolds made via sending a constant $2 \mathrm{~V}$ (Figure 4.5) appear to be more promising. Multiple pores greater than $30 \mu \mathrm{m}$ in diameter can be seen in each image. A few prime examples are highlighted in each via the green scalar lines. A completely accurate portrayal of the porosity of these scaffolds is obscured by the gold-sputtered layer placed on these scaffolds, however, it still is evident that the scaffolds made via the constant $2 \mathrm{~V}$ method produced results closer to what was desired for osteogenic integration due to the more evenly-distributed pores and larger pore sizes.

To further this conclusion, ImageJ was used to determine the amount of pixels in each SEM image that were dark enough to be considered surface pores. This provides quantitative data to the SEM images, however it is still important to note that this does not provide data on the number of pores and size of pores within the image, but rather an estimate on surface porosity.

Figure 4.6 shows a boxplot comparing the amount of pixels determined to be surface pores in each SEM image between the two gas foaming methods. It is evident from this figure that the scaffolds made by sending a constant $2 \mathrm{~V}$ to the positioner tend to have a higher surface porosity. The minimum amount of pixels determined to be surface pores for scaffolds created with the $2 \mathrm{~V}$ method is greater than 200,000 pixels, and the maximum is 425,000 pixels. In contrast, the minimum amount of pixels determined to be surface pores for scaffolds created using the PID is approximately 50,000 pixels, and the maximum is approximately 250,000 pixels. Table 4.4 gives statistical evidence to this comparison of filtered pixel values between SEM images of the scaffolds made in the two different ways via an ANOVA test. The F-ratio is 15.82, which is greater than 4 , and the P-value is 0.0026 , which is less than 0.05 . This allows us to reject the null hypothesis that there is no statistically significant difference between the mean 
amount of pixels in the SEM images determined to be surface pores between the two gas foaming methods and accept the alternative hypothesis that there is a statistically significant difference between the mean amount of pixels in the SEM images determined to be surface pores between the two gas foaming methods.

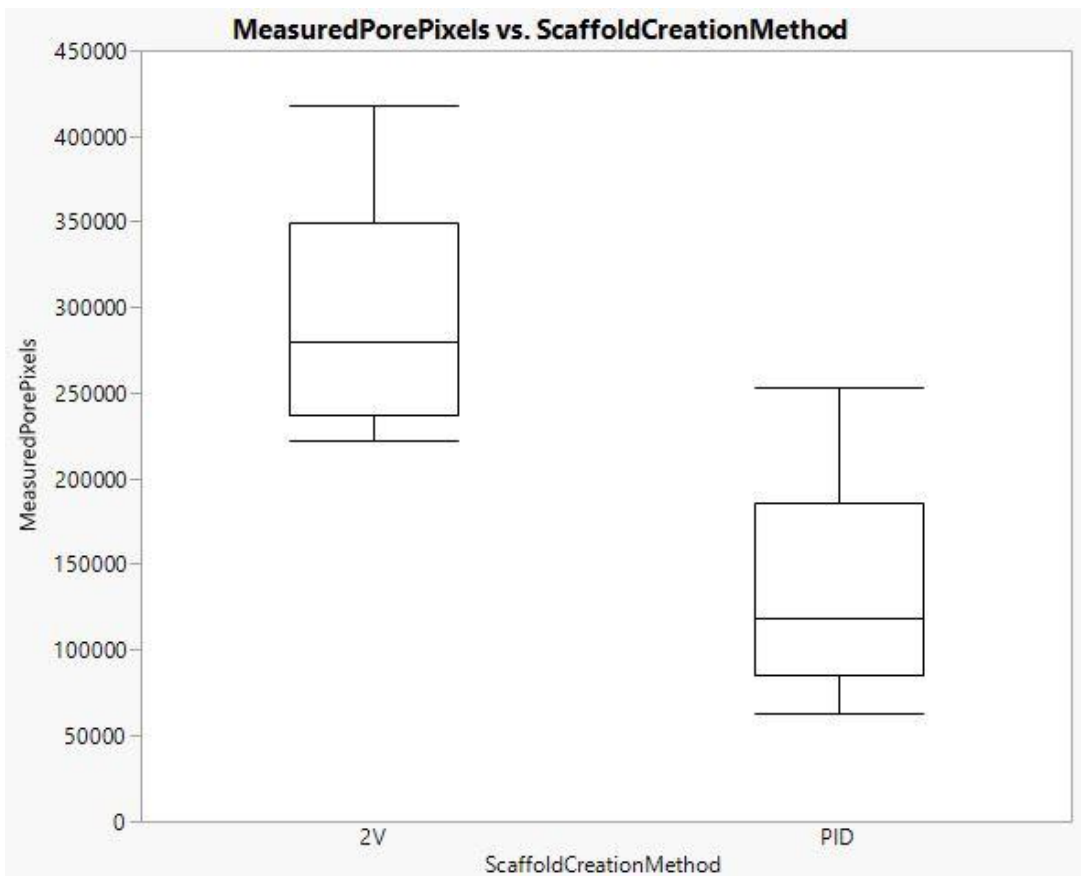

Figure 4.6 Boxplot created in JMP 12 comparing the number of pixels in an SEM image that are considered to be surface pores in the PLGA scaffolds created via the two different gas foaming methods

Table 4.4 Mean values of pixels determined to be pores in ImageJ and the resulting F-stat and P-value from running an ANOVA in JMP 12 on the number of measured pore pixels in the SEM images of scaffolds made via the different gas foaming methods

\begin{tabular}{|c|c|c|c|}
\hline 2V Mean (Pore Pixels) & PID Mean (Pore Pixels) & F-stat & P-value \\
\hline 294643 & 134573 & 15.82 & $\mathbf{0 . 0 0 2 6}$ \\
\hline
\end{tabular}

The combined results from the pressure release graphs and SEM images taken for the two different gas foaming methods show the constant $2 \mathrm{~V}$ method to be the more ideal method of gas foaming pressure release. Using the PID, even with its most ideal PID constants, is unreliable in producing reproducible results in scaffold fabrication. Using the PID instead of the constant $2 \mathrm{~V}$ 
method would be a waste of both materials and time. Most likely, SEM images would need to be taken of one scaffold from each batch of scaffolds fabricated to determine if the gas foaming was successful in producing scaffolds of proper porosity. The constant $2 \mathrm{~V}$ method produces much more consistent results as seen by the extreme difference in the variability of line of best fit constants between Tables 4.1 and 4.2. The SEM images of the scaffolds made via the constant $2 \mathrm{~V}$ method show an interconnected matrix of pores with diameters larger than the necessary $30 \mu \mathrm{m}$ for osteoblast adhesion, whereas the SEM images for the scaffolds made with the PID show a lack of desirable pore sizes and a lack of connectivity of pores. JMP 12 was used to further demonstrate that the surface porosity of the scaffolds made with the constant $2 \mathrm{~V}$ was significantly greater than the surface porosity of scaffolds made with the PID. Due to these results, the PID in the NI LabView program was bypassed and a constant $2 \mathrm{~V}$ was sent to the positioner during gas foaming releases.

\subsection{Discussion Regarding the Contamination}

The growth of osteoblasts onto the PLGA scaffolds was stopped prematurely due to a fungal infection that had contaminated the 48 -well plate. There were four potential reasons for this infection: human error during culture, a medium stain in the incubator, an old bottle of human osteoblast growth medium was used during plating (3 months old), and the aspirating waste container was full past the normal disposal line. The medium stain in the back of the incubator was near the affected flasks of osteoblasts at the time and had what could have been identified as white hairs on it, however this diagnosis is uncertain. The origin of this medium stain is uncertain. The old osteoblast growth medium showed no signs of $\mathrm{pH}$ change, indicated 
by medium color, whereas the contaminated osteoblast-containing medium turned to a yellowish-salmon from its usual red hue, indicating a decrease in $\mathrm{pH}$. It also seems very unlikely that the contamination arose from the aspirating waste container, but it still must be documented as a possibility. Human error in aseptic protocol is also always a potential source for contamination and therefore must be taken into account as well. All potential sources of contamination were taken care of as soon as they were noticed. The growth of osteoblasts onto scaffolds was not restarted due to the expense of both time, which would have been approximately 1.5 months to make new scaffolds and expand osteoblasts, and money, largely due to the price of human osteoblast growth medium. While the fact that osteoblasts were grown on to the scaffolds for only 6 days as opposed to 10 days is unfortunate, any extracellular matrix secreted by the osteoblasts while they were alive could still be determined with Alizarin Red S staining, and any effect on Young's moduli from secreted extracellular matrix components could still be determined through compression testing.

\subsection{Results of Staining}

Alizarin Red S staining was performed to look for calcium in the cryosectioned scaffolds. There were 3 scaffold treatments being looked at: Dulbecco's Phosphate Buffered Saline (dPBS)soaked scaffolds, Human Osteoblast Differentiation Medium (ODM)-soaked scaffolds, and ODM-soaked scaffolds that had osteoblasts deposited on them. The hypothesis was that the scaffolds that had osteoblasts deposited on them would have the most calcium present due to the calcium that would be synthesized by the osteoblasts. The ODM-soaked scaffolds without osteoblasts were thought to potentially have some calcium present as the components of the 
media were nondisclosed to the public, but likely contains calcium to help promote osteogenesis. The dPBS-soaked scaffolds were to serve as a control as calcium is not a component of dPBS, nor was it used in scaffold fabrication.

Figure 4.7 shows the images taken of the different scaffold treatment groups via an inverted optical microscope. As expected, there was no Alizarin Red S staining in the dPBSsoaked scaffolds as there were no calcium-containing components present. There was some staining present in the ODM-soaked scaffolds which was not unexpected. Most of the staining in the ODM-soaked scaffolds was aqueous, however there were a few aggregated stains most noticeably in ODM3. These aggregated stains were even more present in the ODM-soaked scaffolds that had osteoblasts on them. These aggregated stains were more pronounced, stained more heavily, and appeared more frequently than the aggregated stains of the ODM-scaffolds without osteoblasts. Larger versions of the images in Figure 4.7 can be found in Appendix C.

The perceived increase in aggregated clumps of Alizarin Red S-stained material seen in the scaffold cryosections that had osteoblasts deposited on them tells us that, in the 6 days of osteoblast growth on the scaffolds, the osteoblasts deposited calcium on the PLGA matrix. The perceived increase in Alizarin Red S-stained material seen in the scaffolds that had osteoblasts deposited on them should allow us to make correlations of any perceived trend in compression testing data if proven significant. 


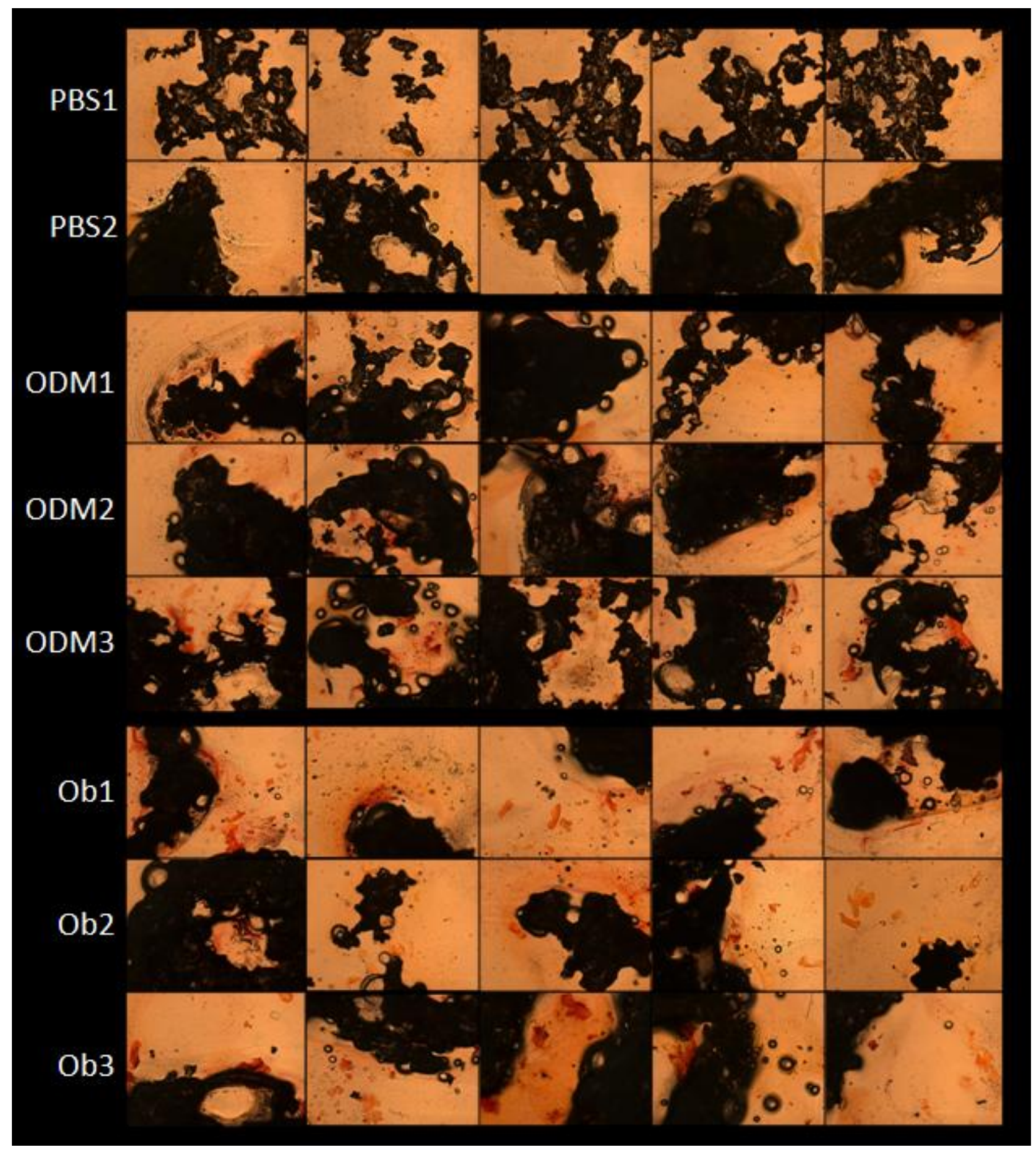

Fig 4.7. Images taken with an inverted optical microscope at $4 x$ magnification of scaffold cryosections stained in Alizarin Red S. There are 2 dPBS-soaked scaffolds labeled as dPBS[scaffold \#]. There are 3ODM-soaked scaffolds labeled as ODM[scaffold \#]. There are 3ODM-soaked scaffolds that had osteoblasts deposited on them labeled as $\mathrm{Ob}$ [scaffold \#]. Components stained red contain calcium. 
Fiji ImageJ was used in an attempt to gain quantitative insight on the trend seen qualitatively by measuring the amount of pixels in the different $4 \mathrm{x}$ magnification inverted optical microscope images that were stained by Alizarin Red S. These values of Alizarin Red S-stained pixels were analyzed in JMP 12 for statistical significance. Figure 4.8 shows a boxplot of the different scaffold treatment groups' Alizarin Red S-stained pixels. The dPBS-soaked scaffolds clearly show much less Alizarin Red S staining than the other two treatment groups. The scaffolds that had osteoblasts deposited on them show on average more Alizarin Red S staining than the ODM-soaked scaffolds without osteoblasts, and one image had significantly more Alizarin Red S-stained pixels (almost 5x as much as the next largest measurement) than the rest of the images. Table 4.5 shows the mean values of total number of pixels in each inverted optical microscope image determined to be Alizarin Red S-stained material in ImageJ and the results of an ANOVA run between all of the treatment groups. The F-statistic of 7.47, which is greater than 4 , and the P-value of 0.0019 , which is less than 0.05 , allows us to reject the null hypothesis that the means between each treatment group are the same (not statistically significant), and accept the alternative hypothesis that at least one of the means is different (statistically significant). Table 4.6 allows us to determine which means are statistically significantly different from the others by performing a Tukey's HSD post-hoc analysis. This analysis compares each treatment group with each treatment group. Table 4.6 shows that the scaffolds that had osteoblasts deposited on them have a statistically significantly larger amount of Alizarin Red S-stained pixels than both the scaffolds that were soaked in ODM without osteoblasts and the dPBSsoaked scaffolds via the P-value less than 0.05. The ODM-soaked scaffolds without osteoblasts and the dPBS-soaked scaffolds are not statistically significantly different. This furthers our belief that that the osteoblasts had a significantly positive effect on the amount of calcium present in 
the PLGA scaffolds. It is important to take into account that the variability in the cryosectioned slices diminishes the complete accuracy of the quantitative results, nonetheless, the extreme difference in Alizarin Red S-stained pixels shown by the JMP 12 output allows us to continue to claim that the osteoblasts deposited on the scaffolds had a significant effect.

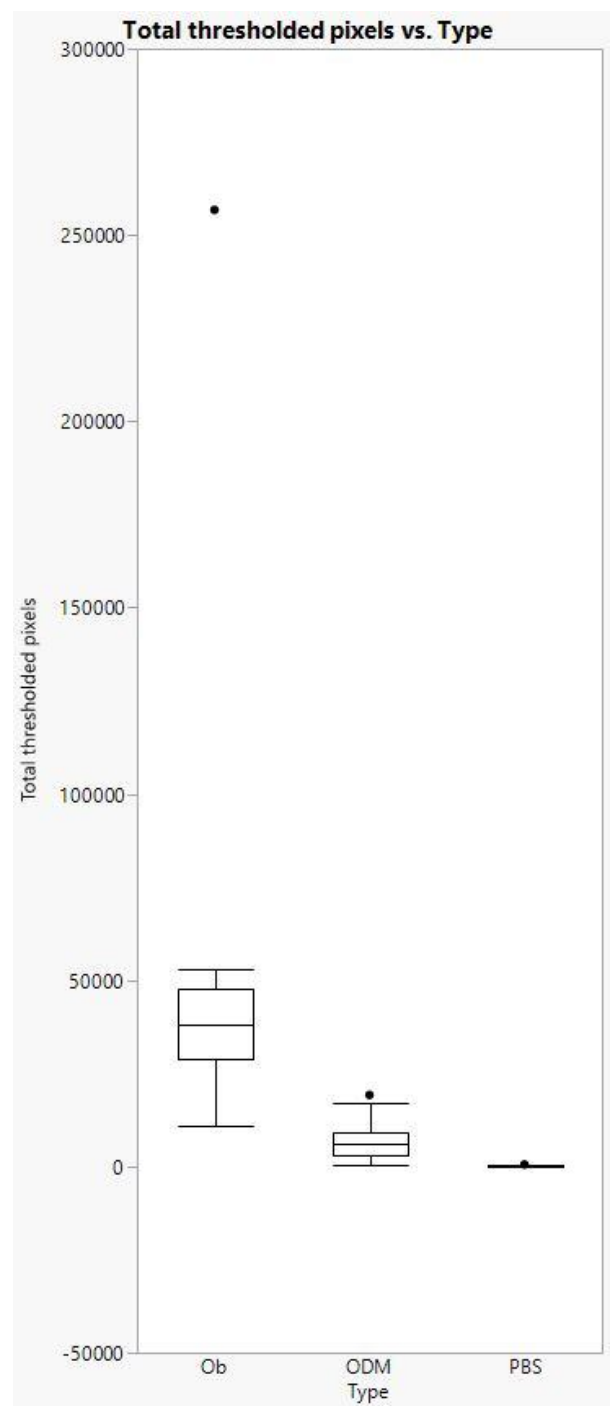

Fig 4.8 Boxplot created in JMP 12 comparing the distribution of amount of Alizarin Red S-stained pixels in each inverted optical microscope image for the three treatment groups 
Table 4.5 Mean values of pixels determined to be Alizarin Red S-stained material in ImageJ and the resulting F-stat and P-value from running an ANOVA in JMP 12

\begin{tabular}{|c|c|c|c|c|}
\hline Ob Mean (ARS pixels) & ODM Mean (ARS pixels) & PBS mean (ARS pixels) & F-stat & P-value \\
\hline 49841 & 7371 & 149 & 7.47 & $\mathbf{0 . 0 0 1 9}$ \\
\hline
\end{tabular}

Table 4.6 P-values from all pairs Tukey's HSD output comparing individual treatment groups with each other for the amount of Alizarin Red S-stained pixels in each inverted optical microscope image

\begin{tabular}{|c|c|}
\hline Comparison & P-value \\
\hline Ob - PBS & 0.0051 \\
\hline Ob - ODM & 0.0075 \\
\hline ODM - PBS & 0.8773 \\
\hline
\end{tabular}

\subsection{Results of Compression Testing}

Compression tests were ran on 16 scaffolds: 4 soaked in Human Osteoblast Differentiation Medium (ODM) that had osteoblasts deposited on them, 4 soaked in ODM without osteoblasts, 4 soaked in Dulbecco's Phosphate Buffered Saline (dPBS), and 4 left dry. Graphs of the compressive load $(\mathrm{N})$ over extension $(\mathrm{mm})$ were created in real time. The Bluehill 3 software also calculated and exported the Young's modulus (MPa), maximum load experienced $(\mathrm{N})$, and load at the ultimate compressive yield point $(\mathrm{N})$ of each scaffold tested.

Figure 4.9 shows graphs of applied compressive load over extension of each scaffold tested. There are no obvious irregularities between the curves between samples or treatment groups. Figure 4.9 seems to show that the scaffolds compressed similarly. There are no yield points as characterized by Figure 2.8 and therefore no values for load at yield were recorded. All of the compression runs set off the condition to terminate the test when $450 \mathrm{~N}$ were reached and as such all the recorded values for maximum load are all around $450 \mathrm{~N}$. The raw data of the compression tests can be seen in Appendix D. 

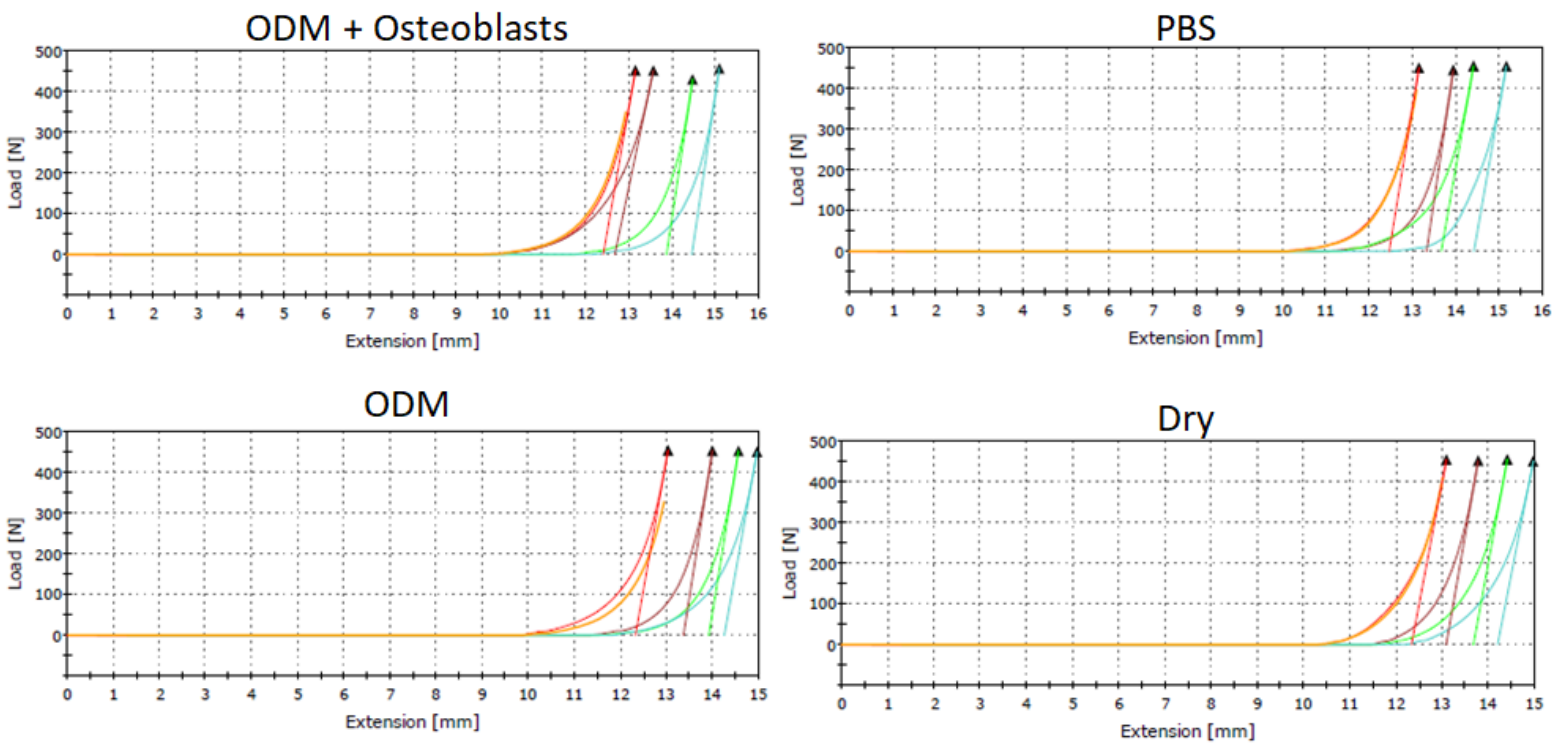

Fig 4.9 The compressive load $(\mathrm{N})$ over extension $(\mathrm{mm})$ of each scaffold in each treatment condition. The orange line represents the first scaffold of a treatment group test, the red is the second, the green is the third, and the light blue is the fourth. The load starts increasing at roughly the same point in extension for each sample in a treatment group, but is shown shifted for visibility.

Table 4.7 shows the Young's moduli for all the scaffolds that underwent compression testing and it shows the mean value and standard deviation for each treatment group. We thought that the second scaffold tested for the scaffolds that had osteoblasts deposited on them might be an outlier due to the way in which it compressed, in which case the mean for the Young's modulus of scaffolds that had osteoblasts deposited on them would be 173.20MPa. The scaffold in question had a slanted top surface as opposed to the other scaffolds' top surfaces which were more parallel to the compression platens. The software, JMP 12 was used to determine if the scaffold was indeed an outlier. A specimen can be determined to be an outlier if it exceeds $1.5 x$ the interquartile range from the first or third quantile (Zinc, 2010). 
Table 4.7 Table of Young's moduli of each scaffold compressed in each treatment group in MPa.

\begin{tabular}{|c|c|c|c|c|c|}
\hline & & Young's M & loduli (MP & \\
\hline & & \multicolumn{4}{|c|}{ Scaffold Type } \\
\hline & & Dry & PBS & ODM & $\mathrm{ODM}+\mathrm{Ob}$ \\
\hline \multirow{4}{*}{$\begin{array}{c}\text { Specimen } \\
\#\end{array}$} & 1 & $\begin{array}{r}153.26 \\
\end{array}$ & 166.63 & \begin{tabular}{|l}
164.20 \\
\end{tabular} & \begin{tabular}{|l}
153.93 \\
\end{tabular} \\
\hline & 2 & 166.48 & 184.53 & 183.41 & 128.73 \\
\hline & 3 & 158.70 & 156.53 & 179.27 & 181.43 \\
\hline & 4 & 148.70 & 154.98 & 161.76 & 184.25 \\
\hline & Mean & 156.79 & 165.67 & 172.16 & 162.09 \\
\hline & Std. Dev. & 7.648685 & 13.5932 & 10.78221 & 26.107403 \\
\hline
\end{tabular}

Table 4.8 shows the interquartile range and the first quantile of the Young's moduli values for compression testing on the osteoblast-containing scaffolds. From this, we can determine that $1.5 x$ the interquartile range from the first quantile is $86.51(135.03$ - 48.52). This means that the value of $128.73 \mathrm{MPa}$ cannot be determined as an outlier, since it is not lower than 86.51. However, that does not necessarily mean that it is not an outlier. With such a small sample size $(n=4)$, every data point has an influential impact on where the quantiles rest, and the Young's modulus value of $128.73 \mathrm{MPa}$ has a very influential impact on the value of the first quantile.

Table 4.8 The interquartile range and first quantile of the Young's moduli values for the compression tests run on the osteoblast-containing scaffolds

\begin{tabular}{|c|c|}
\hline Interquartile Range & First Quantile \\
\hline 48.52 & 135.03 \\
\hline
\end{tabular}

Tables 4.9 and 4.10 show the JMP 12 output for the student's t-test between each pair of scaffold types (indicated by levels) and gives the p-value of each comparison. In these comparisons the null hypothesis is that there is no statistically significant difference between scaffold types and the alternative hypothesis is that there is a statistically significant difference between scaffold types. In both scenarios, with and without the $128.73 \mathrm{MPa}$ value, we fail to 
reject the null hypothesis that there is no statistically significant difference between scaffold types since there is no P-value lower than the $\alpha$ value of 0.05 . Therefore, it does not matter if the $128.73 \mathrm{MPa}$ value is an outlier or not as the difference in mean values for the Young's moduli between scaffold types cannot be proven as statistically significant.

Table 4.9 Individual student's T-test Young's moduli between each pair of scaffold types including the 128.73 value $(\alpha=0.05)$

\begin{tabular}{|c|c|c|}
\multicolumn{4}{|c|}{ Comparison } \\
\hline Type & Type & P-value \\
\hline ODM & ob & 0.3945 \\
\hline ODM & PBS & 0.5798 \\
\hline ODM & Dry & 0.2026 \\
\hline PBS & ob & 0.7589 \\
\hline PBS & Dry & 0.4513 \\
\hline ob & Dry & 0.6505 \\
\hline
\end{tabular}

Table 4.10 Individual student's T-test Young's moduli between each pair of scaffold types excluding the 128.73 value $(\alpha=0.05)$

Comparison

\begin{tabular}{|c|c|c|}
\hline Type & Type & P-value \\
\hline ODM & ob & 0.9129 \\
\hline ODM & PBS & 0.4678 \\
\hline ODM & Dry & 0.1025 \\
\hline PBS & ob & 0.4361 \\
\hline PBS & Dry & 0.3256 \\
\hline ob & Dry & 0.106 \\
\hline
\end{tabular}

The small sample size used is another factor towards why statistical significance cannot be proved. This can be illustrated by the equation for standard error. The standard error for a sample statistic indicates the precision of the sample mean of a given population (Diong, 2016). In this case, the standard error indicates the precision of the mean value for the Young's modulus of each scaffold treatment. Standard error is equal to the standard deviation of a set of data over the square root of the sample size. Therefore, larger sample sizes produce smaller standard 
errors, which in turn produce smaller confidence intervals, increasing the chance of attaining a statistically significant p-value (CASC, 2018).

Even though the results of the compression testing were shown to be inconclusive, the possibility that the presence of osteoblasts strengthens the material stiffness of PLGA has not been disproven. Past experiments have shown that osteoblasts should increase the stiffness of the material they are grown on as they increase mineral volume on that material (Turner, 2006). The growth of osteoblasts onto the scaffolds was stopped prematurely and looking at the Young's moduli without the questionable scaffold compression data point shows a higher Young's modulus (173.20MPa) than any of the other treatment conditions. However, the values of Young's moduli received from our compression testing do approximate literature values seen for the Young's modulus of trabecular bone. Values for the Young's modulus of native trabecular bone have been reported to range from 1MPa to 199.5MPa (Lakatos, Magyar, \& Bojtár, 2014). All of the scaffolds' Young's moduli fall within the range of native trabecular bone and therefore our scaffold fabrication process shows promise in replicating the material properties of trabecular bone tissue. It may be beneficial to attempt to measure some other material property or multiple different material properties to gain further insight on the ability of gas foaming to create scaffolds that replicate native trabecular bone. The Young's moduli values from compression testing all fall within the range of trabecular bone, making determining statistical significance a challenge in whether the presence of osteoblasts make these gas foamed scaffolds more similar to trabecular bone or not. 


\section{Chapter 5}

\section{SUMMARY AND CONCLUSIONS}

This experiment intended to determine if the presence of osteoblasts made PLGA scaffolds more similar to native trabecular bone. The results of compression testing failed to show any statistically significant difference in stiffness via the Young's moduli for any of the scaffold treatments. However, there are multiple factors involved in this lack of statistical significance. For one, the growth of osteoblasts onto the PLGA scaffolds was stopped prematurely due to a fungal infection that had contaminated the 48 -well plate. The small sample size of the scaffolds tested in compression testing was another influential factor in the lack of statistical significance between Young's moduli for each treatment. One scaffold that had osteoblasts deposited on it had a calculated Young's modulus that was much smaller in comparison to the other scaffolds that had osteoblasts deposited on them. This can possibly be attributed to the irregular nature of the top surface of that particular scaffold, where the top surface was slanted as opposed to parallel with compression platens, affecting the way in which the scaffold was compressed. However, the scaffold in question cannot be proven as an outlier largely due to the small sample size of $n=4$. While there is no statistically significant difference in Young's moduli between the different treatment groups, all of the values fall within the literature value range for native trabecular bone of 1MPa to 199.5MPa (Lakatos et al., 2014).

This experiment also intended to determine if gas foaming could be utilized to create scaffolds for bone regenerative therapies. Our test for calcification showed an increased expression of calcium-containing components on the scaffolds that had osteoblasts deposited on 
them by a more defined and larger presence of Alizarin Red S-stained components. This provides evidence that osteoblasts can be grown on gas foamed PLGA scaffolds and that the osteoblasts will secrete calcium-phosphate crystals onto the PLGA scaffold.

The pressure vessel on the California Polytechnic State University of San Luis Obispo campus has now been modified and improved for the purpose of gas foaming scaffolds for bone tissue engineering. This project sets the guidelines on how gas foaming polymer scaffolds can be accomplished and it is flexible enough to be modified for various needs. The NI LabView software created to control the gas pressure release valve can either send a constant voltage to the valve positioner or make use of a programmed Proportional-Integral-Derivative controller. Making use of either of these with different parameters may better suit different polymers and tests will need to be done to determine which method with what parameters is necessary to produce the desired gas foaming results for a specific project.

This project determined that using the NI LabView program to send a constant $2 \mathrm{~V}$ to the positioner produced the most desirable results for gas foaming PLGA to produce scaffolds for osteoblast growth. The observed porosity of these scaffolds showed an interconnected matrix of pores with diameters larger than $30 \mu \mathrm{m}$, the diameter shown to best promote osteogenic activity in the presence of bone-forming cells (Halai et al., 2014). The best PID constants for producing gas foamed PLGA scaffolds were determined to be $\mathrm{Kp}=0.056, \mathrm{Ki}=20,000$, and $\mathrm{Kd}=0.44$, however, even under these conditions, sending a constant $2 \mathrm{~V}$ to the positioner proved to create more consistent gas foaming results with desirable pore sizes.

In future projects, it would be beneficial to try different salt to PLGA ratios to determine if a particular ratio produced more desirable results than the ratio of $80 \%$ salt and $20 \%$ PLGA we 
used. We used this ratio since we feared, if less PLGA was used, the PLGA particles would not be close enough together to properly fuse when gas foamed. However, we believe the scaffolds could be even more porous as that would provide more sites for osteoblasts to adhere to. The overall substrate stiffness should decrease in the gas foamed scaffolds, but the presence of osteoblasts may have a more noticeable effect on stiffness due to having more sites for cell integration. This experiment will need to be redone to achieve the full 10 days of osteoblast growth on to the scaffolds and we suggest attempting to use a 90\% salt and 10\% PLGA ratio for increased porosity. It would also be interesting to test different copolymers such as PLGA/hydroxyapatite to determine if these copolymers better promote osteogenic activity than pure PLGA scaffolds and to record how these copolymers respond to the gas foaming method. There are two reasons hydroxyapatite would be interesting to include as a substrate material. One, the senior project that used a flask of the osteoblasts we grew showed increase osteoblast vitality in the presence of osteoblasts. Two, $50 \%$ of the volume of human bone is made up of hydroxyapatite crystals (Junqueira \& Carneiro, 2005). In future experiments, it could also be beneficial to increase the cell seeding density of osteoblasts onto individual scaffolds as one million osteoblasts was an arbitrary value picked for this experiment. It also would be beneficial to look into different methods of mechanically testing the scaffolds for similarities to native trabecular bone as measuring stiffness via compression testing did not provide statistically significant results and all the Young's moduli for the different scaffolds fell within the range of trabecular bone already.

This experiment tested two things: the ability and efficiency of using gas foaming to create PLGA scaffolds for cell deposition and whether or not the presence of osteoblasts on PLGA scaffolds made the scaffolds more similar to trabecular bone. Gas foaming was proven to 
be a useful and innovative tool in the world of bone tissue engineering to create PLGA scaffolds, and it was shown that osteoblasts successfully secreted extracellular matrix components onto the scaffolds. The presence of osteoblasts did not have a statistically significant effect on the stiffness of the PLGA scaffolds, but the Young's modulus values found did fall in the range of literature values for native trabecular bone furthering the evidence of gas foaming being a practical method of creating scaffolds for tissue engineering. Gas foaming was proven to be an effective, novel method of creating scaffolds for bone tissue engineering, but the presence of osteoblasts was not shown to increase the scaffolds' stiffness to that of native trabecular bone. 


\section{BIBLIOGRAPHY}

Ammrf. (2014). Troubleshooting: edge effect, charging, sample damage. Retrieved from http://www.ammrf.org.au/myscope/sem/practice/principles/troubleshooting.php

Avgoustakis, K. (2005). Polylactic-Co-Glycolic Acid ( PLGA ). Encyclopedia of Biomaterials and Biomedical Engineering, (Scheme 2), 1-11. https://doi.org/10.1081/E-EBBE120013950

Bhardwaj, N., \& Kundu, S. C. (2010). Electrospinning: A fascinating fiber fabrication technique. Biotechnology Advances. https://doi.org/10.1016/j.biotechadv.2010.01.004

Blair, H. C., Larrouture, Q. C., Li, Y., Lin, H., Beer-Stoltz, D., Liu, L., ... Nelson, D. J. (2017). Osteoblast Differentiation and Bone Matrix Formation In Vivo and In Vitro. Tissue Engineering Part B: Reviews, 23(3), 268-280. https://doi.org/10.1089/ten.teb.2016.0454

Brighton, C. T., \& Hunt, R. M. (1997). Early Histologic and Ultrastructural Changes in Microvessels of Periosteal Callus. Journal of Orthopaedic Trauma, 11(4), 244-253. https://doi.org/10.1097/00005131-199705000-00002

Byon, C. H., Sun, Y., Chen, J., Yuan, K., Mao, X., Heath, J. M., ... Chen, Y. (2011). Runx2upregulated receptor activator of nuclear factor $\mathrm{\kappa b}$ ligand in calcifying smooth muscle cells promotes migration and osteoclastic differentiation of macrophages. Arteriosclerosis, Thrombosis, and Vascular Biology, 31(6), 1387-1396. https://doi.org/10.1161/ATVBAHA.110.222547

CASC. (2018). The Relationship Between Confidence Intervals and p-values. Retrieved from http://www.ucl.ac.uk/ich/short-courses-events/about-stats-courses/statsrm/Chapter_6_Content/relationship_confintervals_pvalues

Diong, J. (2016). STATISTICS NOTE: HOW DOES SAMPLE SIZE AFFECT PRECISION OF ESTIMATES? Retrieved from https://scientificallysound.org/2016/03/03/how-does-samplesize-affect-precision-of-estimates/

Felton, G. P. (2011). Biodegradable polymers : processing, degradation, and applications. Retrieved from https://books.google.com.ua/books?id=KutjAQAACAAJ\&dq=Biodegradable_Polymers\&hl =ru\&sa=X\&ved=0ahUKEwjn-Zip75rUAhWsIJoKHe_6BMQQ6AEIOTAD

Figari, P. (2015). STEPS TO ANALYZING A MATERIAL'S PROPERTIES FROM ITS STRESS/STRAIN CURVE. Retrieved from http://www.instructables.com/id/Steps-toAnalyzing-a-Materials-Properties-from-its/

Gentile, P., Chiono, V., Carmagnola, I., \& Hatton, P. V. (2014). An overview of poly(lactic-coglycolic) Acid (PLGA)-based biomaterials for bone tissue engineering. International Journal of Molecular Sciences. https://doi.org/10.3390/ijms15033640

Halai, M., Ker, A., Meek, R. D., Nadeem, D., Sjostrom, T., Su, B., ... Young, P. S. (2014). Scanning electron microscopical observation of an osteoblast/osteoclast co-culture on 
micropatterned orthopaedic ceramics. Journal of Tissue Engineering, 5, 204173141455211. https://doi.org/10.1177/2041731414552114

Hansma, P. (2004). AFM and SEM Imaging of Bone. Retrieved from https://hansmalab.physics.ucsb.edu/afmbone.html

Harding, T. (2018). PLGA. California Polytechnic University of San Luis Obispo.

Harris, L. D., Kim, B. S., \& Mooney, D. J. (1998). Open pore biodegradable matrices formed with gas foaming. Journal of Biomedical Materials Research, 42(3), 396-402. https://doi.org/10.1002/(SICI)1097-4636(19981205)42:3<396::AID-JBM7>3.0.CO;2-E

Hasegawa, T., Yamamoto, T., Tsuchiya, E., Hongo, H., Tsuboi, K., Kudo, A., ... Amizuka, N. (2017). Ultrastructural and biochemical aspects of matrix vesicle-mediated mineralization. Japanese Dental Science Review. https://doi.org/10.1016/j.jdsr.2016.09.002

Höflinger, G. (2013). Brief Introduction to Coating Technology for Electron Microscopy. Retrieved from http://www.leica-microsystems.com/science-lab/brief-introduction-tocoating-technology-for-electron-microscopy/

Junqueira, L., \& Carneiro, J. (2005). Basic Histology: Text \& Atlas. Statrefcom, 544.

Kang, S. W., La, W. G., \& Kim, B. S. (2009). Open macroporous poly(lactic-co-glycolic acid) microspheres as an injectable scaffold for cartilage tissue engineering. Journal of Biomaterials Science, Polymer Edition, 20(3), 399-409. https://doi.org/10.1163/156856209X412236

Knospe, C. (2006). PID control. Control Systems, IEEE, 26(1), 216-251. https://doi.org/10.1109/MCS.2006.1580151

Lakatos, É., Magyar, L., \& Bojtár, I. (2014). Material properties of the mandibular trabecular bone. Journal of Medical Engineering, 2014. https://doi.org/10.1155/2014/470539

Li, M., He, P., Wu, Y., Zhang, Y., Xia, H., Zheng, Y., \& Han, Y. (2016). Stimulatory effects of the degradation products from $\mathrm{Mg}-\mathrm{Ca}-\mathrm{Sr}$ alloy on the osteogenesis through regulating ERK signaling pathway. Scientific Reports, 6. https://doi.org/10.1038/srep32323

Liao, C. J., Chen, C. F., Chen, J. H., Chiang, S. F., Lin, Y. J., \& Chang, K. Y. (2002). Fabrication of porous biodegradable polymer scaffolds using a solvent merging/particulate leaching method. Journal of Biomedical Materials Research, 59(4), 676-681. https://doi.org/10.1002/jbm.10030

Liu, X., \& Ma, P. X. (2004). Polymeric scaffolds for bone tissue engineering. Annals of Biomedical Engineering, 32(3), 477-486. https://doi.org/10.1023/B:ABME.0000017544.36001.8e

MacVarish, N. K. (2017). The Difference Between Amorphous \& Semi-crystalline Polymers. Retrieved from http://blog.impactplastics-ct.com/blog/the-difference-between-amorphoussemi-crystalline-polymers 
Ngai, K. L., Floudas, G., Plazek, D. J., \& Rizos, A. K. (2002). Amorphous Polymers. Encyclopedia of Polymer Science and Technology, 5, 63-111. https://doi.org/10.1002/0471440264.pst018

Palin, E., Liu, H., \& Webster, T. J. (2005). Mimicking the nanofeatures of bone increases boneforming cell adhesion and proliferation. Nanotechnology. https://doi.org/10.1088/09574484/16/9/069

Polo-Corrales, L., Latorre-Esteves, M., \& Ramirez-Vick, J. E. (2014). Scaffold Design for Bone Regeneration. Journal of Nanoscience and Nanotechnology, 14(1), 15-56. https://doi.org/10.1166/jnn.2014.9127

Rho, J. Y., Ashman, R. B., \& Turner, C. H. (1993). Young's modulus of trabecular and cortical bone material: Ultrasonic and microtensile measurements. Journal of Biomechanics, 26(2), 111-119. https://doi.org/10.1016/0021-9290(93)90042-D

RTP. (2016). Semi-Crystalline Polymers. Retrieved from https://www.rtpcompany.com/products/high-temperature/semi-crystalline-polymers/

Savage, R. (2017). Electron Microscopy.

Shin, C. S., \& Kim, K. M. (2015). The risks and benefits of calcium supplementation. Endocrinology and Metabolism (Seoul, Korea), 30(1), 27-34. https://doi.org/10.3803/EnM.2015.30.1.27

Tanaka-Kamioka, K., Kamioka, H., Ris, H., \& Lim, S. S. (1998). Osteocyte shape is dependent on actin filaments and osteocyte processes are unique actin-rich projections. Journal of Bone and Mineral Research, 13(10), 1555-1568. https://doi.org/10.1359/jbmr.1998.13.10.1555

Turner, C. H. (2006). Bone Strength: Current Concepts. Orthopaedic Research Laboratories and Biomechanics and Biomaterials Research Center, Indiana University Purdue University.

Ultra Epsom. (2010). Ultra Epsom Salt.

Väänänen, H. K., Zhao, H., Mulari, M., \& Halleen, J. M. (2000). The cell biology of osteoclast function. J Cell Sci, 113 ( Pt 3, 377-381. Retrieved from http://www.ncbi.nlm.nih.gov/pubmed/10639325

Zinc, R. (2010). Identifying multivariate inliers and outliers. Retrieved from https://community.jmp.com/t5/JMP-Blog/Identifying-multivariate-inliers-and-outliers/bap/30227 


\section{APPENDIX A: DETERMINING THE PROPORTIONAL-INTEGRAL-DERIVATIVE CONSTANTS}

The most crucial consideration when gas foaming is the rate of carbon dioxide release from the gas foaming chamber. During a successful gas release, a thermodynamic instability occurs, fusing the polymer particles together. To create a repeatable process for releasing the valve, a National Instruments LabView program was created, shown in Figure A.1. This program reads the pressure inside the gas foaming chamber from a pressure transducer connected to a National Instruments USB-6002 Data Acquisition board (DAQ). The program also sends out a voltage through the DAQ to a positioner on the actuated release valve (Valworx \#565255), and the voltage sent out, ranging from 0 to $10 \mathrm{~V}$, determines the position of the release valve. Originally, a Proportional-Integral-Derivative controller (PID) was used to create a reproducible method of gas release, but it was later discarded when it was found that just sending $2 \mathrm{~V}$ to the positioner yielded the desired pressure release in a more reproducible manner. However, it is still important to mention that this PID program exists if future projects plan to make use of the gas foaming chamber and sending a constant $2 \mathrm{~V}$ to the actuated valve doesn't produce desired foaming results. More information on how a PID works is detailed in Section 2.4.

For this experiment, the most optimum PID constants were found to be $\mathrm{Kp}=0.056, \mathrm{Ki}=$ 20,000 , and $\mathrm{Kd}=0.44$. The range of voltages the PID sent to the DAQ to the actuator valve was $1.5 \mathrm{~V}$ to $2.25 \mathrm{~V}$. The set point was made to decrease pressure from 800 psi to 0 linearly over the course of 30 seconds. Figures A.2-A.8 and Tables A.1 and A.2 showcase the pressure releases over time with these values and with changing each value by $1 \%$ in either direction to demonstrate that these values are the most optimum values to get the process variable to follow the set point. 

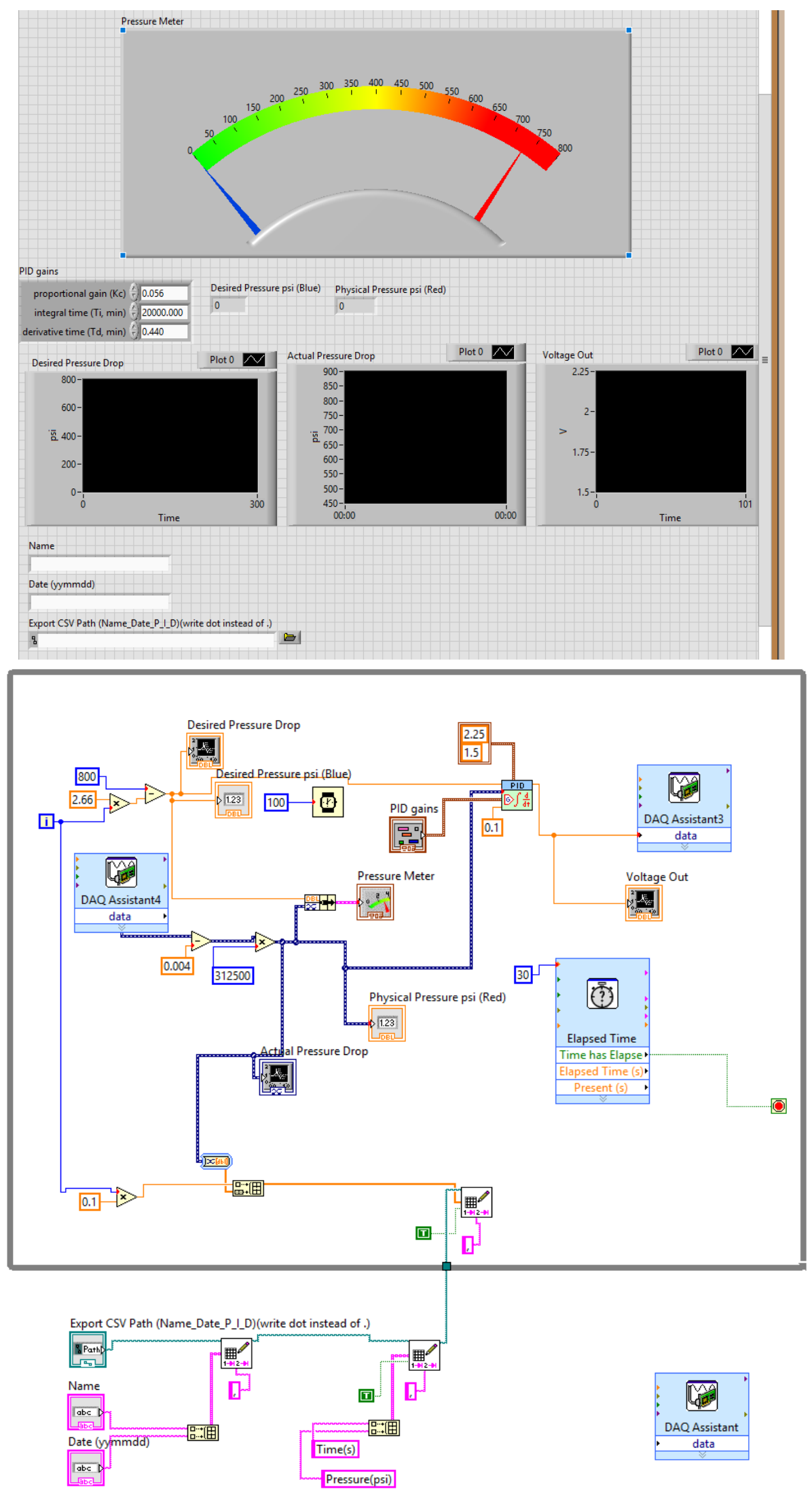

Figure A.1 NI LabView program of PID controller for controlling actuated release valve on gas foaming apparatus 

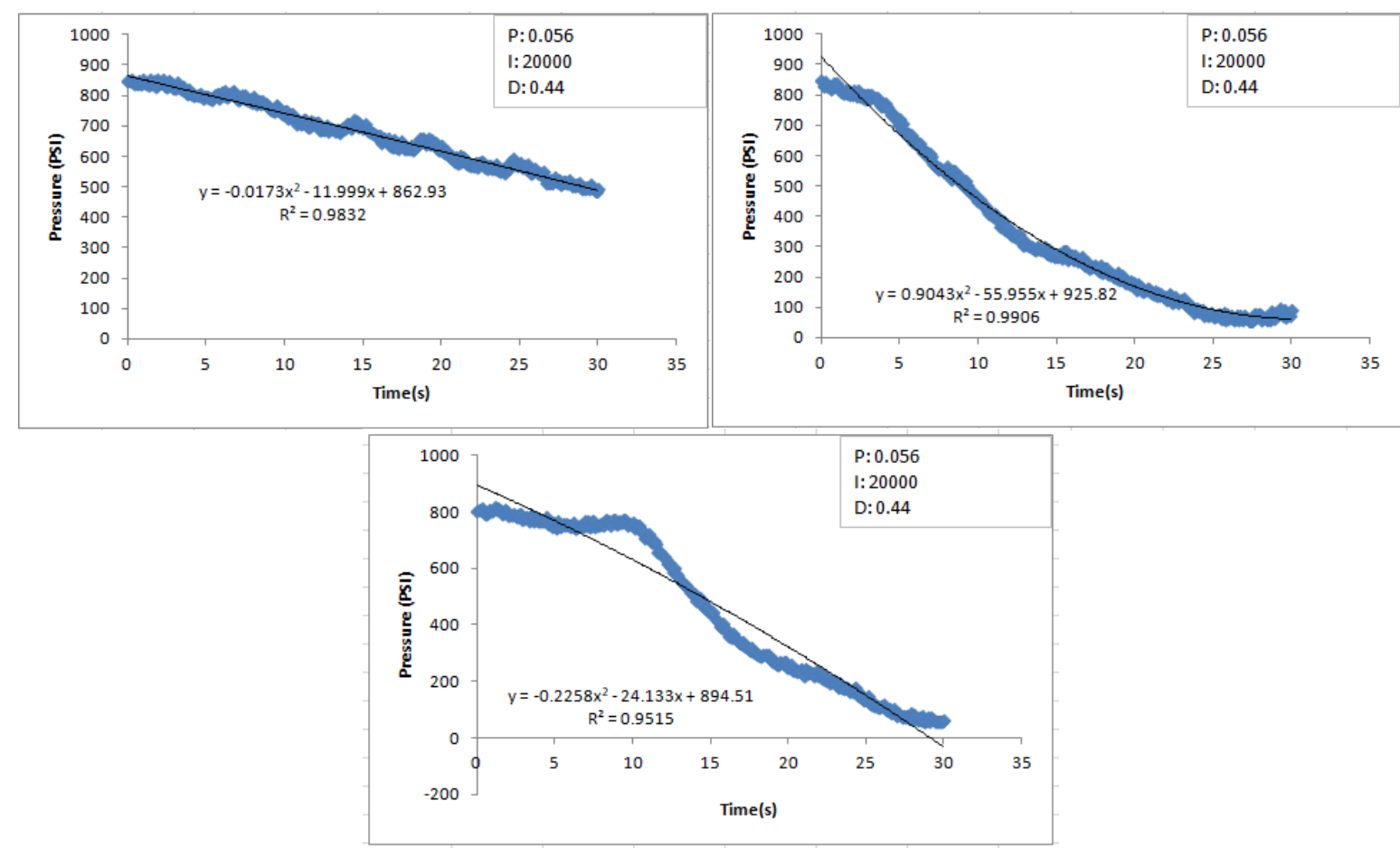

Figure A.2 Three repetitions of gas release over 30s with a PID setting of $\mathrm{P}=0.056, \mathrm{I}=20000, \mathrm{D}=0.44$ (Chosen PID settings)
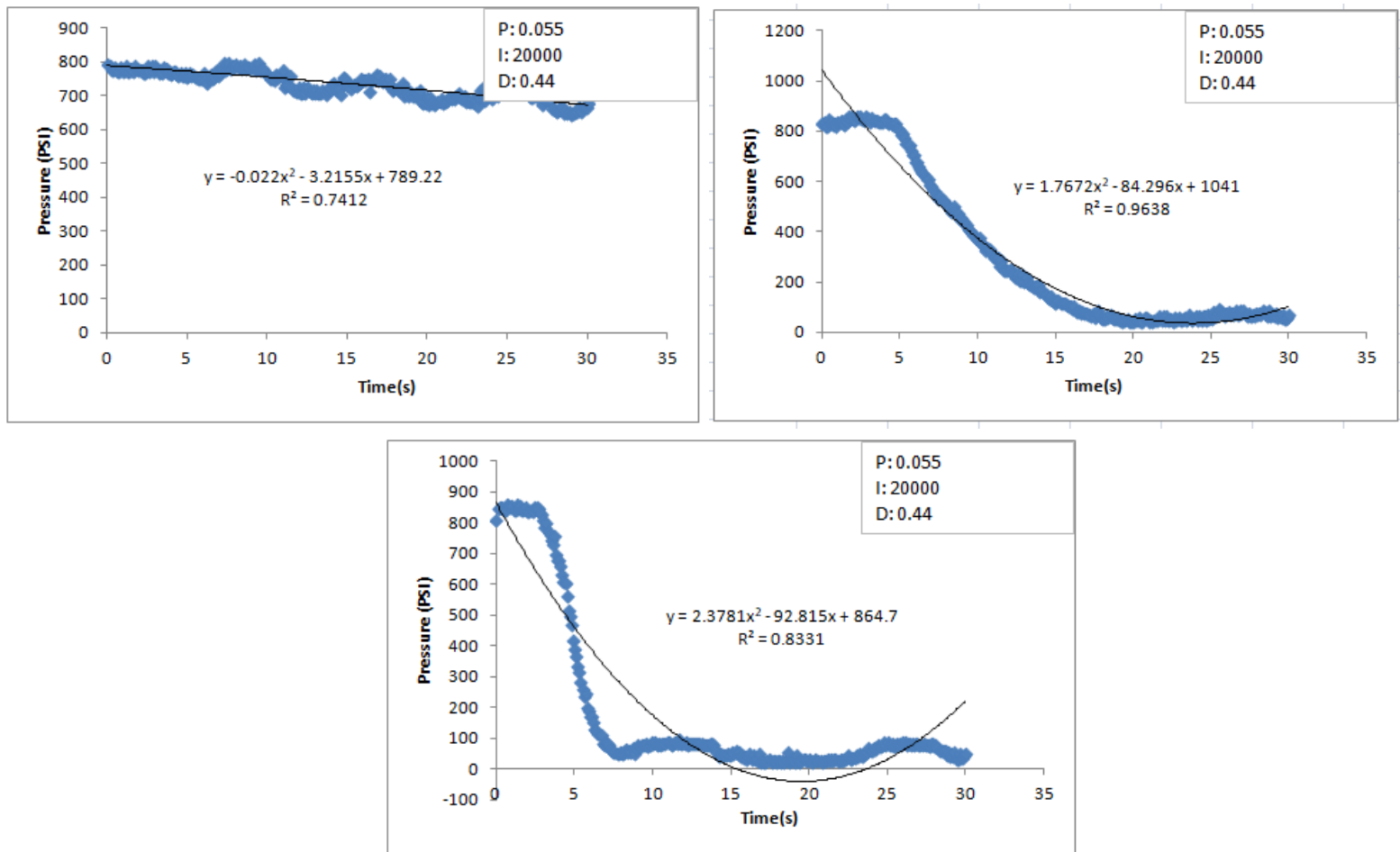

Figure A.3 Three repetitions of gas release over 30s with a PID setting of $\mathrm{P}=0.055, \mathrm{I}=20000, \mathrm{D}=0.44(-1 \% \mathrm{P})$ 

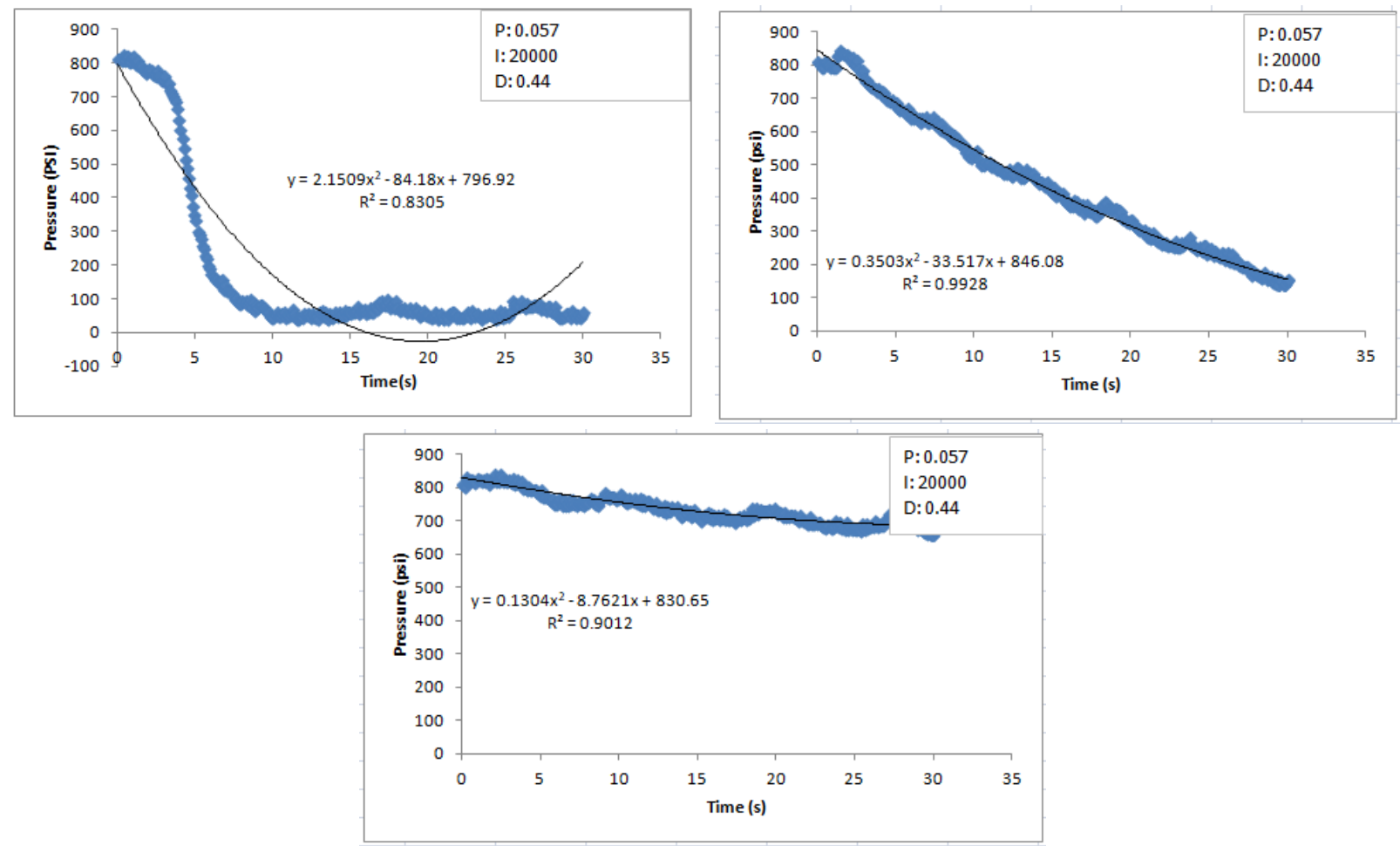

Figure A.4 Three repetitions of gas release over 30 s with a PID setting of $\mathrm{P}=0.057, \mathrm{I}=20000, \mathrm{D}=0.44(+1 \% \mathrm{P})$
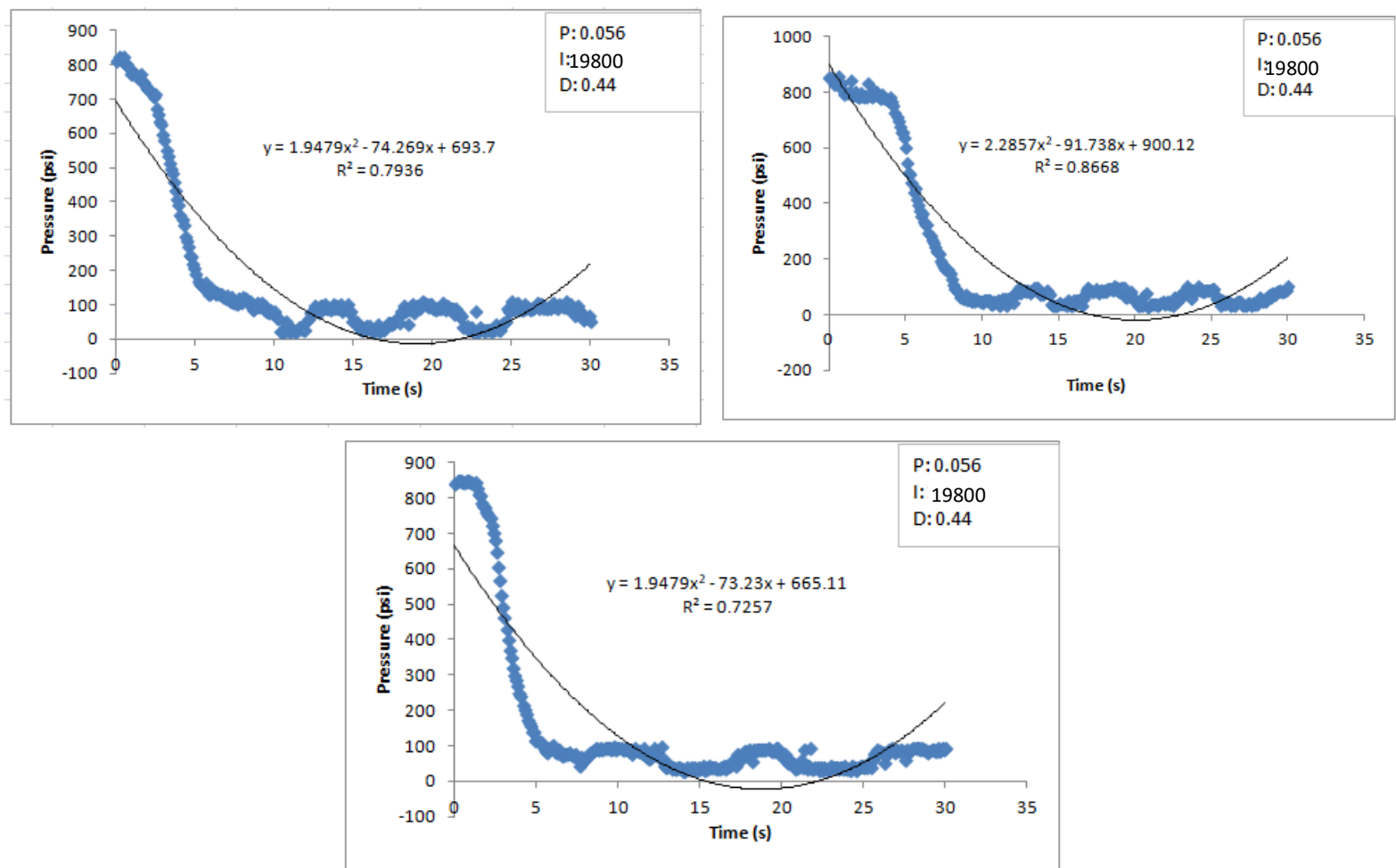

Figure A.5 Three repetitions of gas release over 30s with a PID setting of $\mathrm{P}=0.056, \mathrm{I}=19800, \mathrm{D}=0.44(-1 \% \mathrm{I})$ 

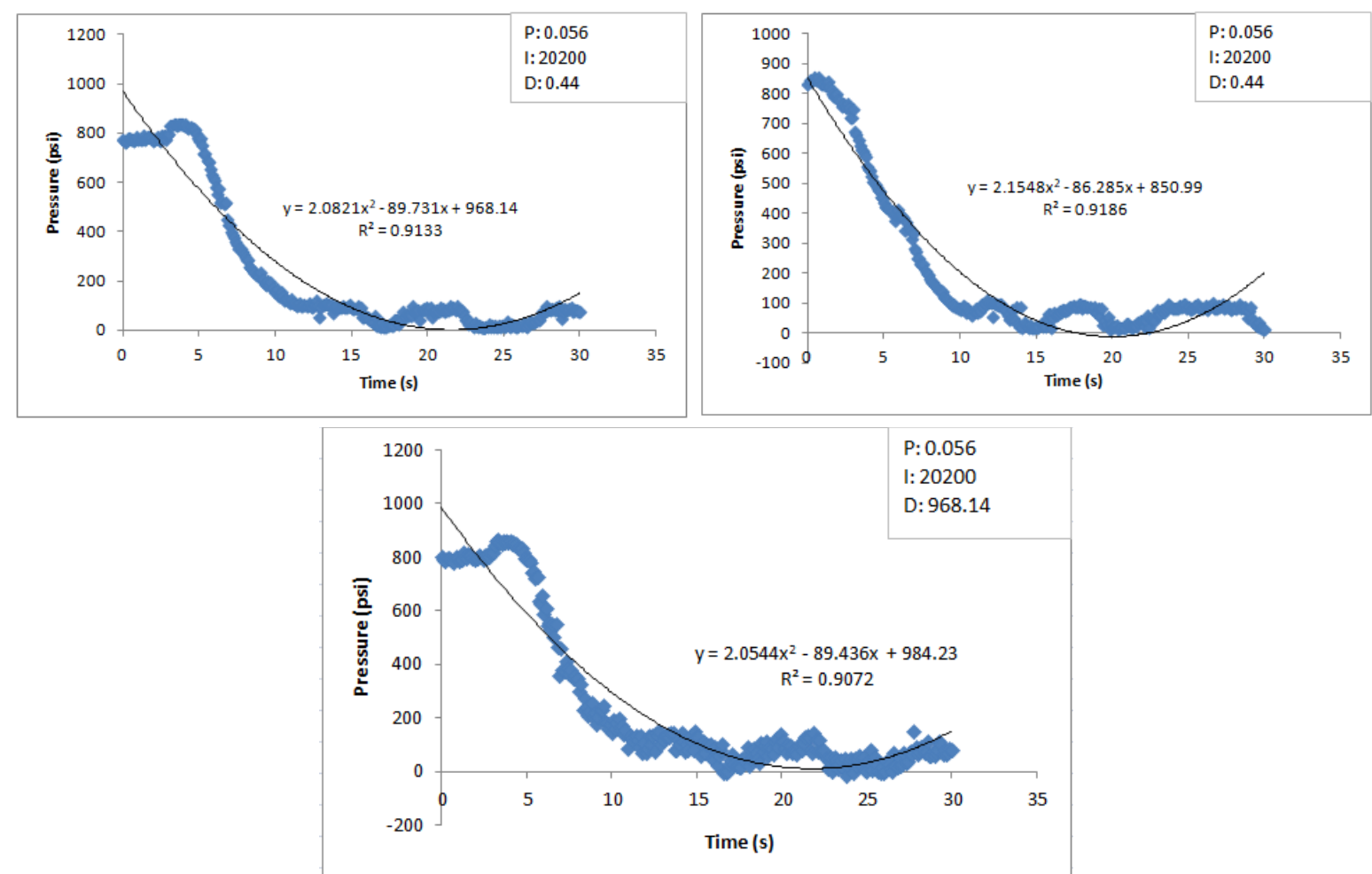

Figure A.6 Three repetitions of gas release over 30s with a PID setting of $\mathrm{P}=0.056, \mathrm{I}=20200, \mathrm{D}=0.44(+1 \% \mathrm{I})$
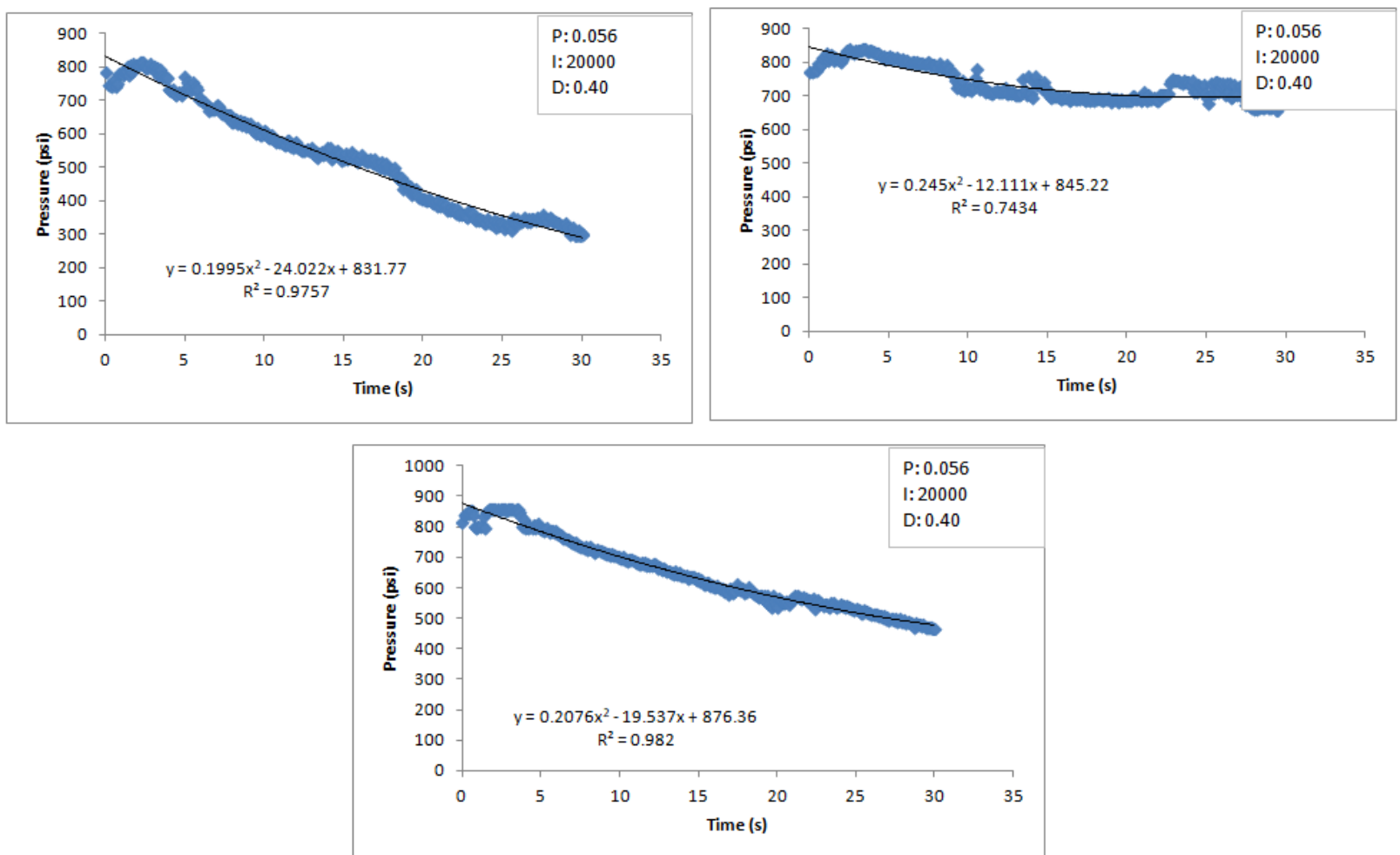

Figure A.7 Three repetitions of gas release over 30s with a PID setting of $\mathrm{P}=0.056, \mathrm{I}=20000, \mathrm{D}=0.40(-1 \% \mathrm{D})$ 

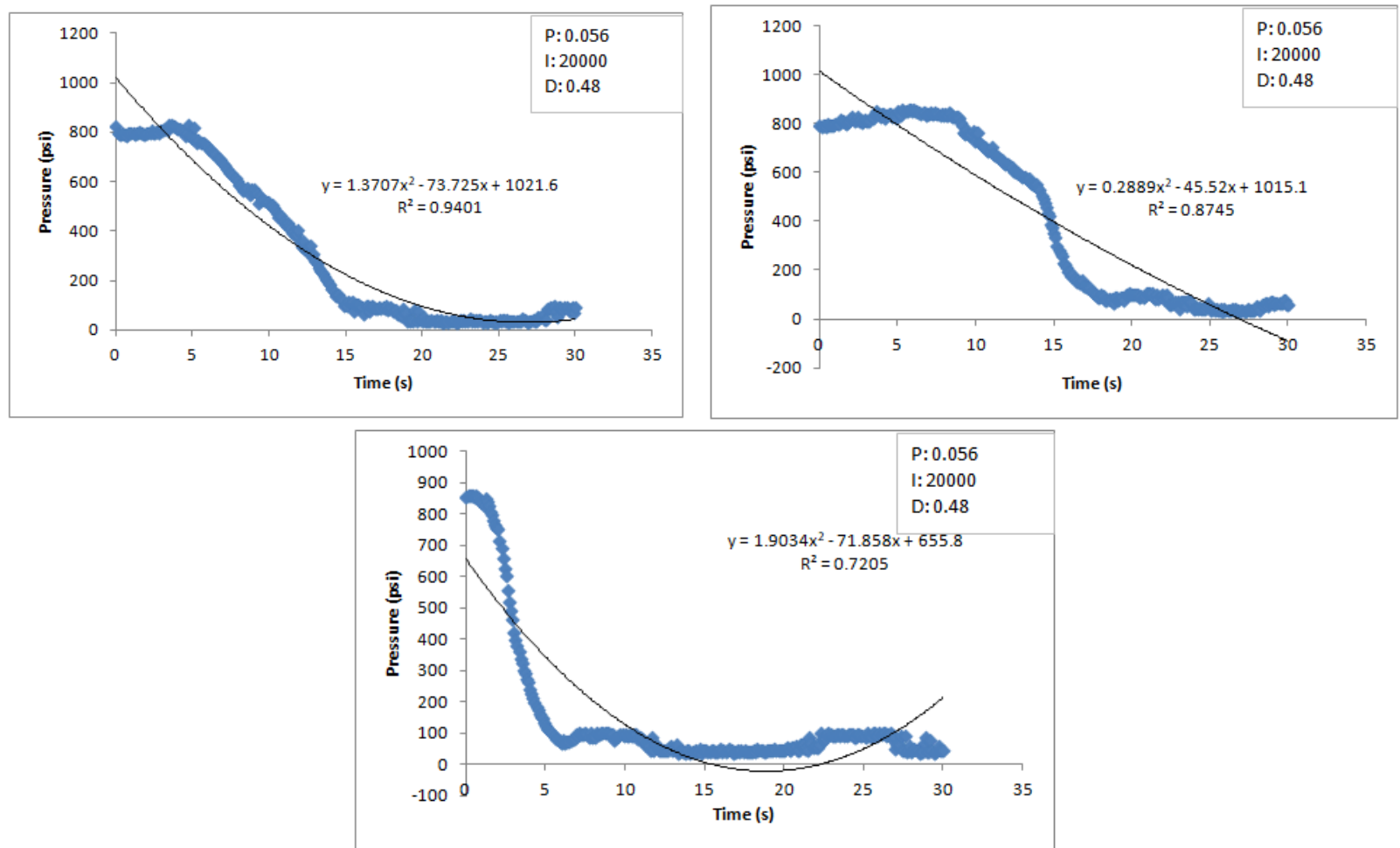

Figure A.8 Three repetitions of gas release over 30s with a PID setting of $P=0.056, I=20000, D=0.48(+1 \% D)$ 
Table A.1 Summarized line-of-best-fit values from each PID run in Figures A.2 - A.8

\begin{tabular}{|c|c|c|c|c|c|c|c|}
\hline & $\mathbf{P}$ & $I$ & D & $x^{\wedge} 2$ & $x$ & c & $\mathrm{R}^{\wedge} \mathbf{2}$ \\
\hline & Ideal & Ideal & Ideal & 0 & -26.7 & 800 & 1 \\
\hline \multirow{3}{*}{ Chosen } & 0.056 & 20000 & 0.44 & -0.02 & -12.00 & 862.90 & 0.9883 \\
\hline & 0.056 & 20000 & 0.44 & 0.90 & -55.96 & 925.82 & 0.9906 \\
\hline & 0.056 & 20000 & 0.44 & -0.23 & -24.13 & 894.51 & 0.9515 \\
\hline \multirow{3}{*}{ "-1\% P" } & 0.055 & 20000 & 0.44 & -0.02 & -3.22 & 789.22 & 0.7412 \\
\hline & 0.055 & 20000 & 0.44 & 1.77 & -84.30 & 1041.00 & 0.9638 \\
\hline & 0.055 & 20000 & 0.44 & 2.38 & -92.82 & 864.70 & 0.8331 \\
\hline \multirow{3}{*}{ "+1\% P" } & 0.057 & 20000 & 0.44 & 2.15 & -84.18 & 796.92 & 0.8305 \\
\hline & 0.057 & 20000 & 0.44 & 0.35 & -33.52 & 846.08 & 0.9928 \\
\hline & 0.057 & 20000 & 0.44 & 0.13 & -8.76 & 830.65 & 0.9012 \\
\hline \multirow{3}{*}{ "-1\% I" } & 0.056 & 19800 & 0.44 & 1.95 & -74.27 & 693.70 & 0.7936 \\
\hline & 0.056 & 19800 & 0.44 & 2.29 & -91.74 & 900.12 & 0.8668 \\
\hline & 0.056 & 19800 & 0.44 & 1.95 & -73.23 & 665.11 & 0.7257 \\
\hline \multirow{3}{*}{ "+1\% I" } & 0.056 & 20200 & 0.44 & 2.08 & -89.73 & 968.14 & 0.9133 \\
\hline & 0.056 & 20200 & 0.44 & 2.15 & -86.29 & 850.99 & 0.9186 \\
\hline & 0.056 & 20200 & 0.44 & 2.05 & -89.44 & 984.23 & 0.9072 \\
\hline \multirow{3}{*}{ "-1\% D" } & 0.056 & 20000 & 0.40 & 0.20 & -24.02 & 831.77 & 0.9757 \\
\hline & 0.056 & 20000 & 0.40 & 0.25 & -12.11 & 845.22 & 0.7434 \\
\hline & 0.056 & 20000 & 0.40 & 0.21 & -19.54 & 876.36 & 0.982 \\
\hline \multirow{3}{*}{$"+1 \%$ D" } & 0.056 & 20000 & 0.48 & 1.37 & -73.73 & 1021.60 & 0.9401 \\
\hline & 0.056 & 20000 & 0.48 & 0.29 & -45.52 & 1015.10 & 0.8745 \\
\hline & 0.056 & 20000 & 0.48 & 1.90 & -71.86 & 655.80 & 0.7205 \\
\hline
\end{tabular}

Table A.2 Average line-of-best-fit values from PID runs in Figures A.2 - A.8 and their percent difference from the ideal values.

\begin{tabular}{|c|c|c|c|c|c|c|}
\hline $\mathbf{P}$ & I & D & $x^{\wedge} 2$ & $\mathbf{x}$ & c & \\
\hline Ideal & Ideal & Ideal & 0 & -26.7 & 800 & \\
\hline \multirow[t]{2}{*}{0.056} & 20000 & 0.44 & 0.22 & -30.70 & 894.41 & Avg. \\
\hline & & & $\mathrm{N} / \mathrm{A}$ & $14.97 \%$ & $11.80 \%$ & $\%$ diff from ideal \\
\hline \multirow[t]{2}{*}{0.055} & 20000 & 0.44 & 1.38 & -60.11 & 898.31 & Avg. \\
\hline & & & $\mathrm{N} / \mathrm{A}$ & $125.14 \%$ & $12.29 \%$ & $\%$ diff from ideal \\
\hline \multirow[t]{2}{*}{0.057} & 20000 & 0.44 & 0.88 & -42.15 & 824.55 & Avg. \\
\hline & & & N/A & $57.88 \%$ & $3.07 \%$ & $\%$ diff from ideal \\
\hline \multirow[t]{2}{*}{0.056} & 19800 & 0.44 & 2.06 & -79.75 & 752.98 & Avg. \\
\hline & & & N/A & $198.68 \%$ & $-5.88 \%$ & $\%$ diff from ideal \\
\hline \multirow[t]{2}{*}{0.056} & 20200 & 0.44 & 2.09 & -88.49 & 934.45 & Avg. \\
\hline & & & N/A & $231.41 \%$ & $16.81 \%$ & $\%$ diff from ideal \\
\hline \multirow[t]{2}{*}{0.056} & 20000 & 0.40 & 0.22 & -18.56 & 851.12 & Avg. \\
\hline & & & $\mathrm{N} / \mathrm{A}$ & $-30.50 \%$ & $6.39 \%$ & $\%$ diff from ideal \\
\hline \multirow[t]{2}{*}{0.056} & 20000 & 0.48 & 1.19 & -63.70 & 897.50 & Avg. \\
\hline & & & N/A & $138.59 \%$ & $12.19 \%$ & $\%$ diff from ideal \\
\hline
\end{tabular}


The NI LabView program was set up such that the PID tried to match the actual pressure release (process variable) to a linear decline from 800 psi to 0 over 30s (set point), shown as the ideal values in Tables A.1 and A.2. Table A.2 demonstrates the PID values of $\mathrm{P}=0.056, \mathrm{I}=$ 20,000 , and $\mathrm{D}=0.44$ as the most ideal PID constants within $1 \%$ of their values. Changing either the P constant, I constant, or D constant by $1 \%$ in the positive or negative direction results in a further derivation from the set point. The fitted lines of best fit were second-degree polynomials and the set point was a linear decline, therefore, the $\mathrm{x}^{2}$ term in the line of best fit closest to 0 was most ideal. The smallest percent difference from the set point for the $\mathrm{x}$ term and constant term for the line of best fit were also the most ideal. Of the tested values in Table A.2, the PID constant terms set to $\mathrm{P}=0.056, \mathrm{I}=20,000$, and $\mathrm{D}=0.44$, yielded the closest results on average to the set point. 


\section{APPENDIX B: GAS FOAMING PRESSURE RELEASE GRAPHS}

There were five iterations of gas foaming during scaffold fabrication. Six scaffolds were gas foamed at a time, resulting in 30 scaffolds created in total. Figures B.1-B.5 show the pressure releases for each gas foam over time.

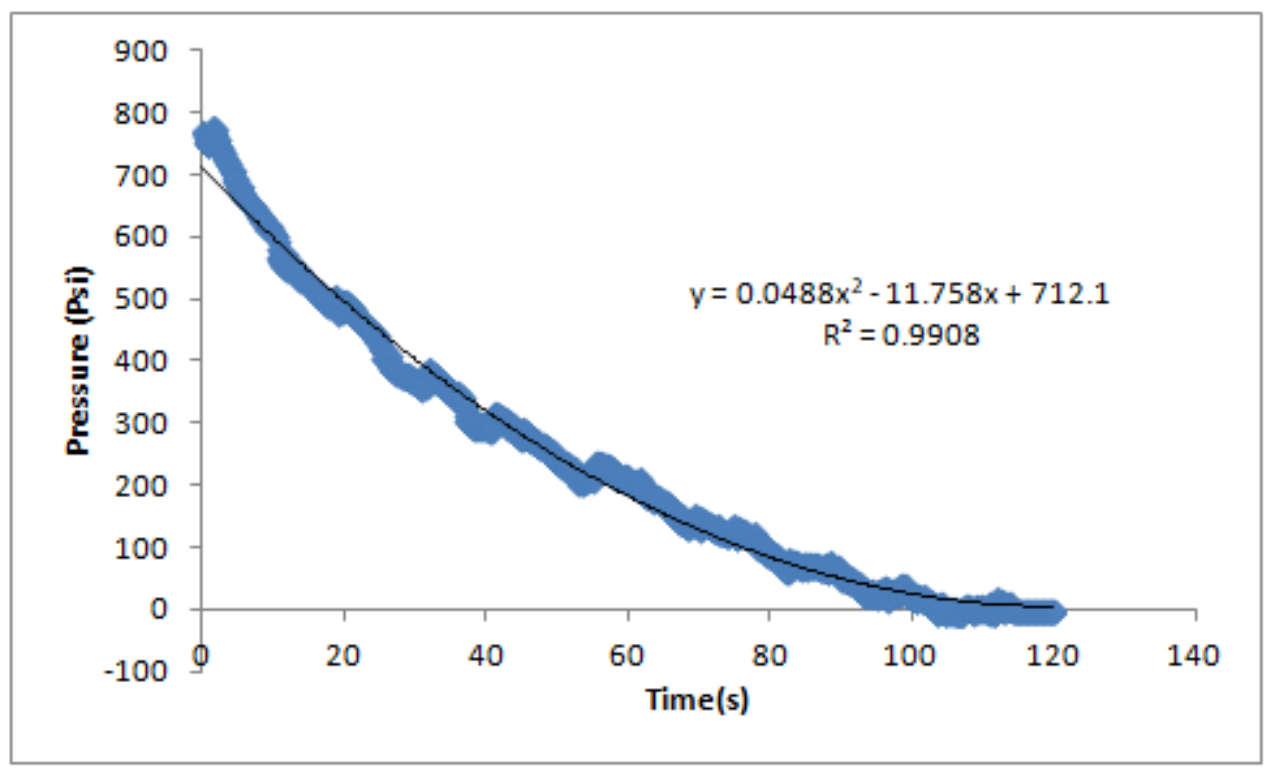

Fig. B.1 Gas foaming pressure release preformed on 05/10/2018

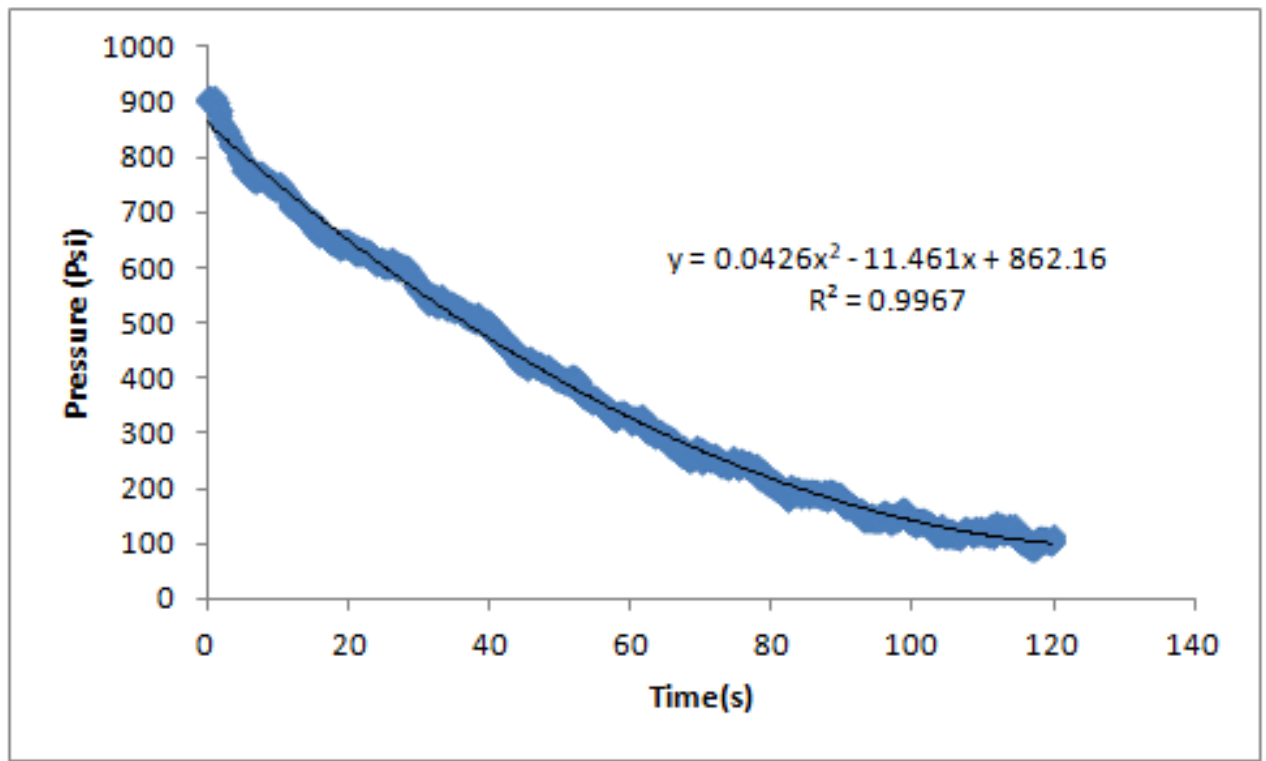

Fig. B.2 Gas foaming pressure release preformed on 05/11/2018 


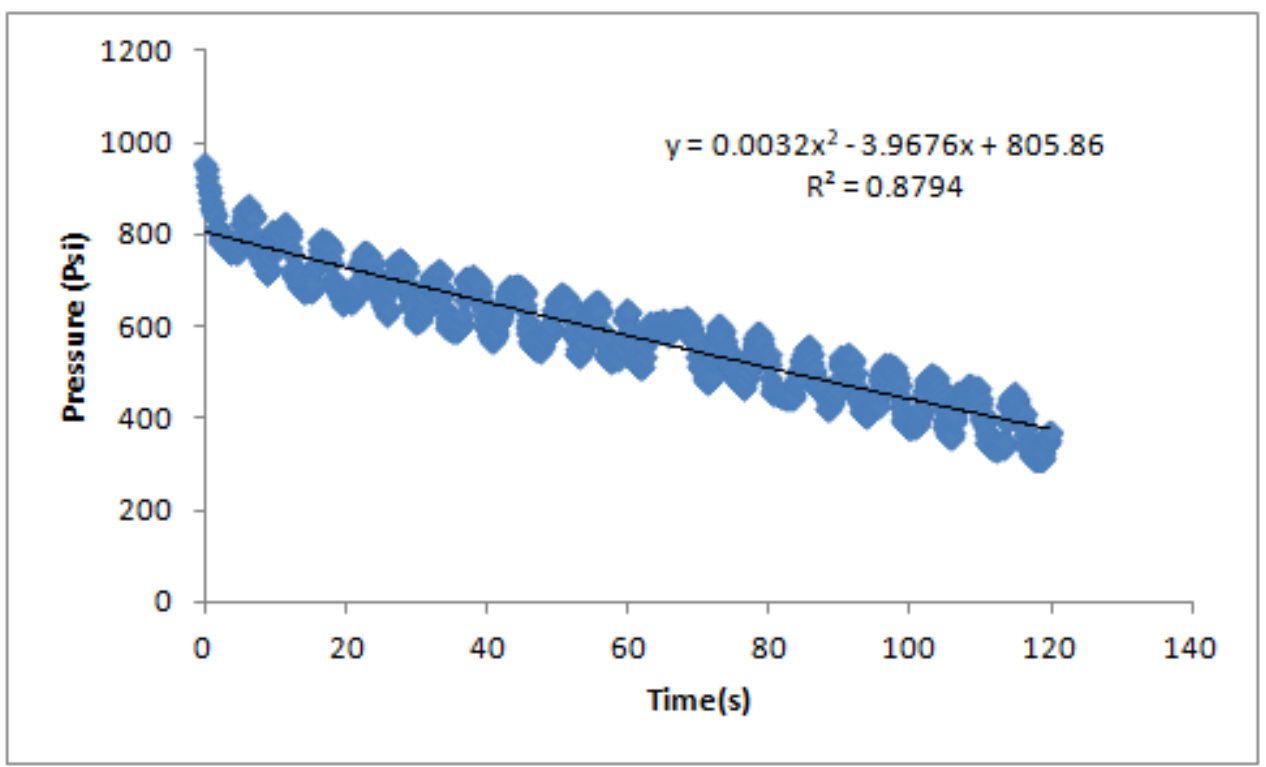

Fig. B.3 Gas foaming pressure release preformed on 05/12/2018

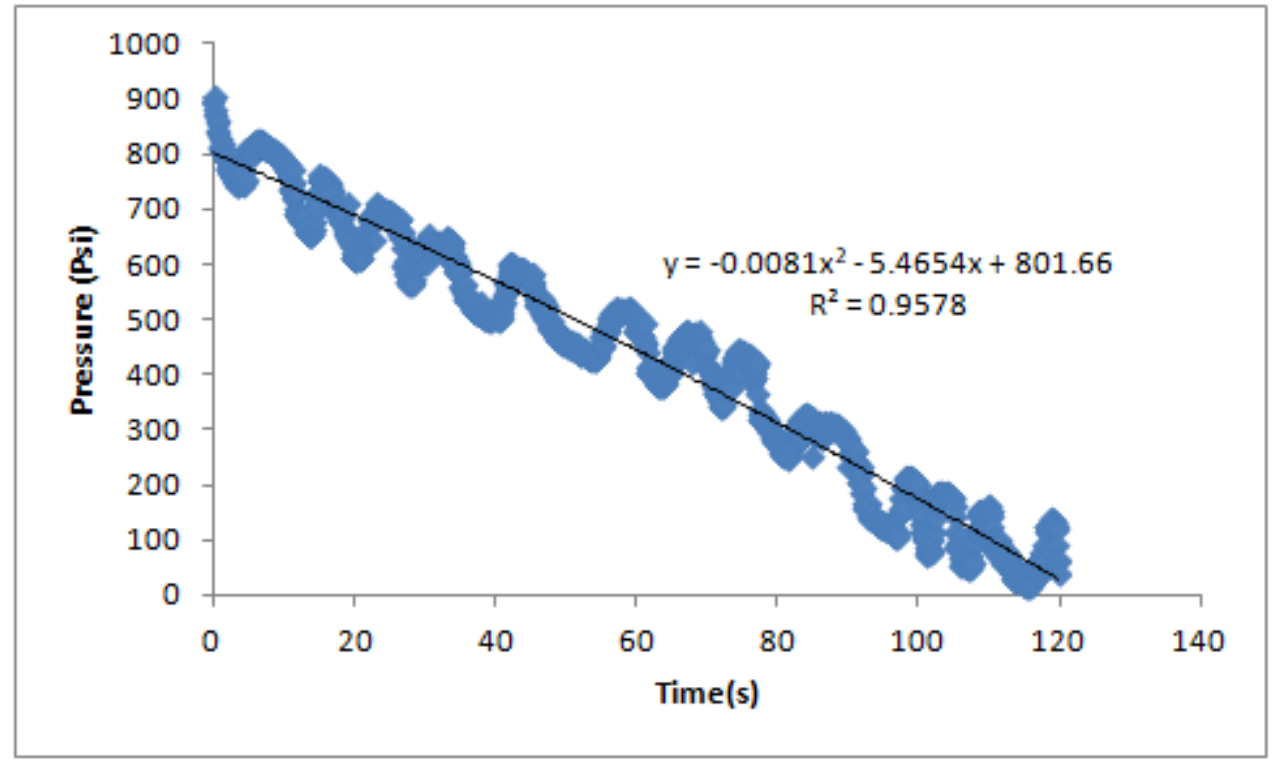

Fig. B.4 Gas foaming pressure release preformed on 05/14/2018 


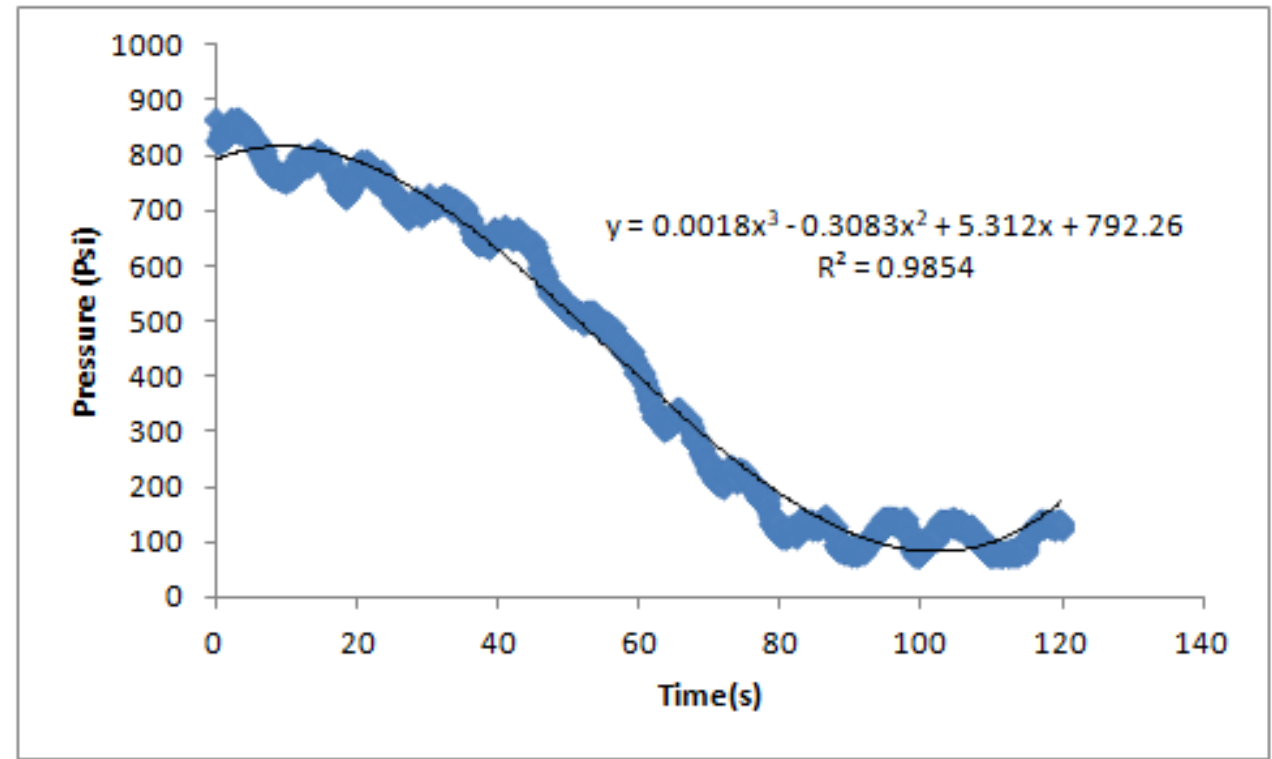

Fig. B.5 Gas foaming pressure release preformed on 05/15/2018 
Figure 4.7 shows the results of the Alizarin Red S staining of scaffold cryosections. The same images from that figure are displayed here but larger for closer analysis.

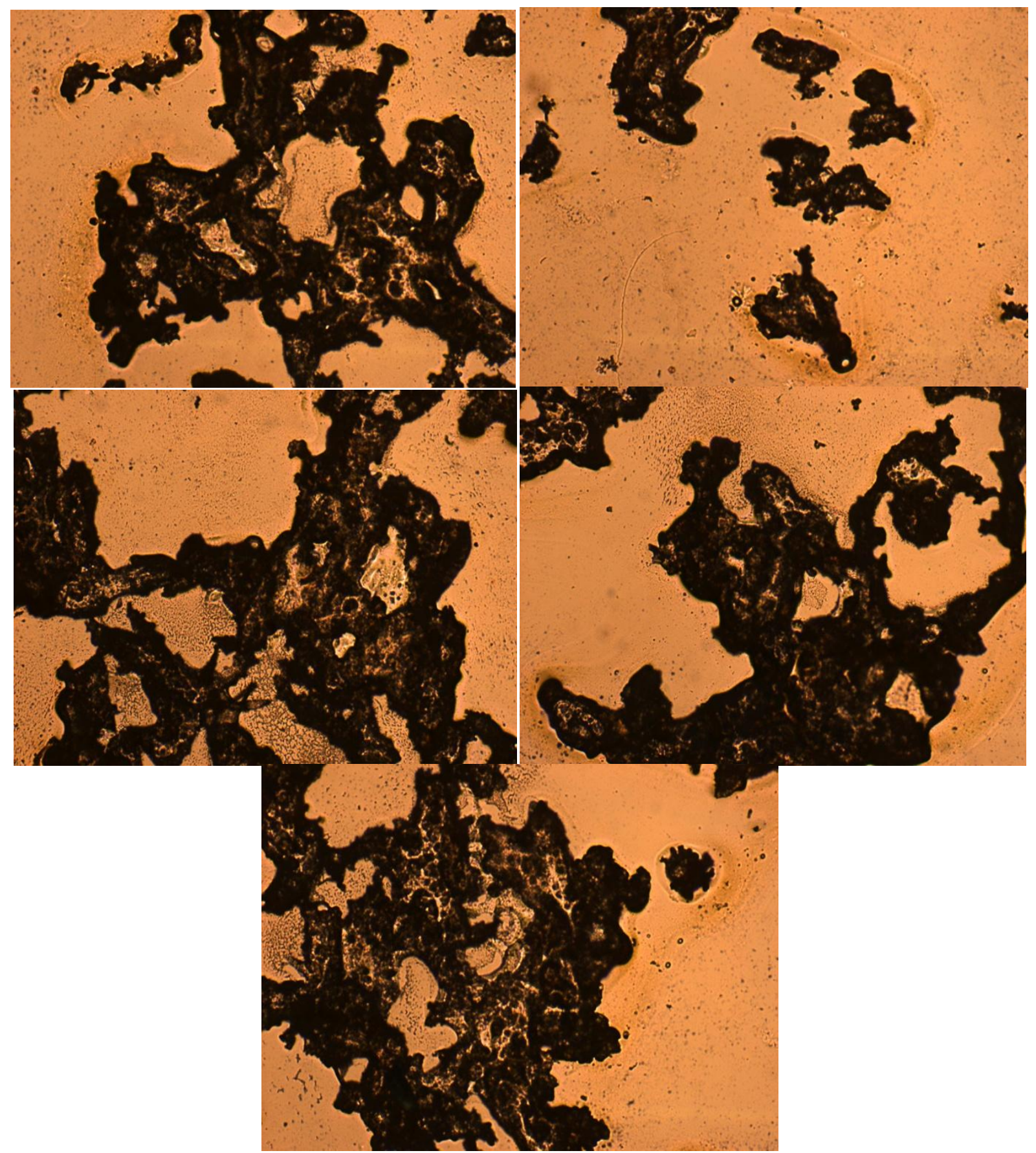

Fig C.1 Five inverted optical microscope images of Alizarin Red S-stained dPBS-soaked scaffold \#1 cryosections taken at $4 \mathrm{x}$ magnification 


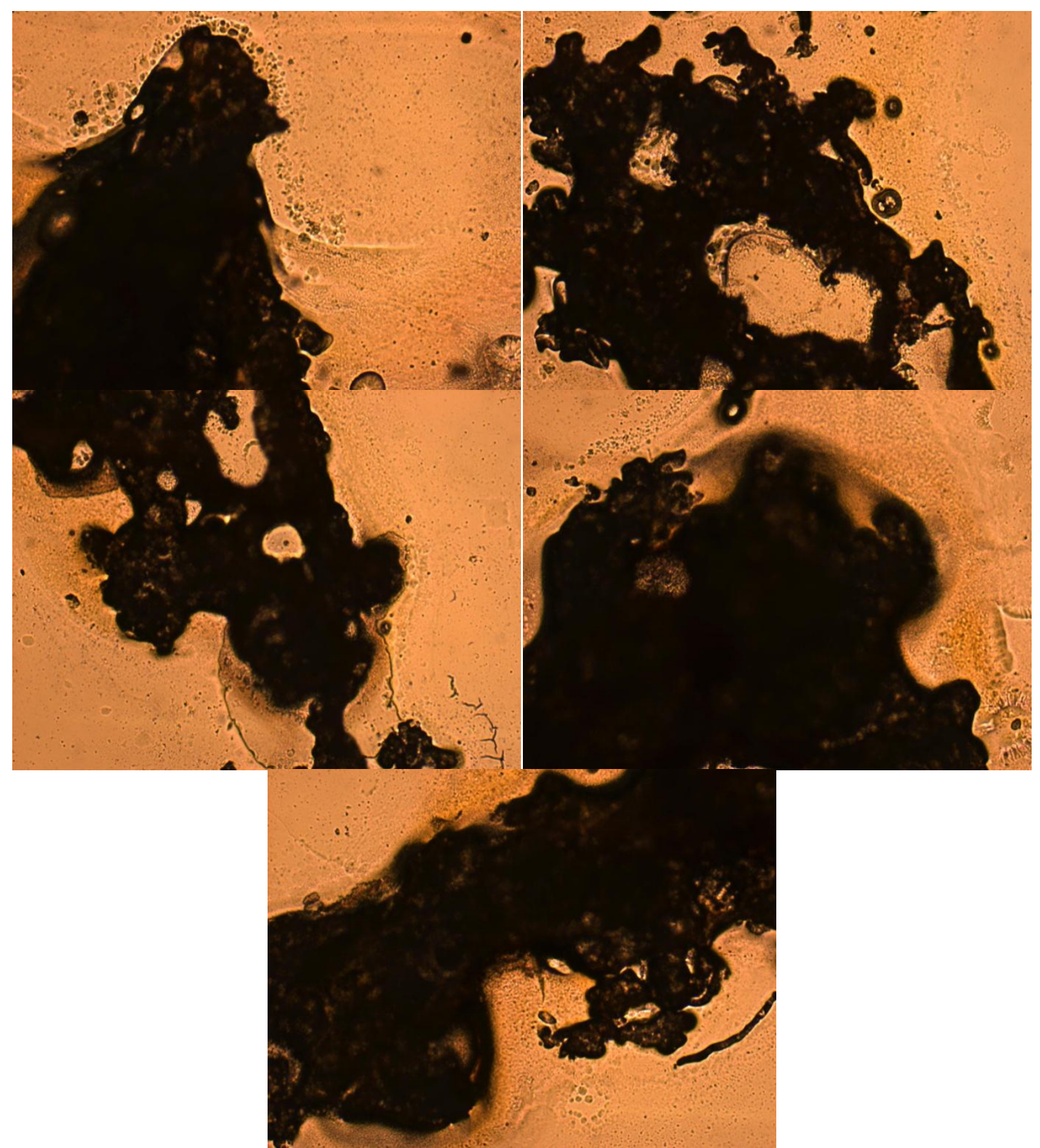

Fig C.2 Five inverted optical microscope images of Alizarin Red S-stained dPBS-soaked scaffold \#2 cryosections taken at $4 \mathrm{x}$ magnification 


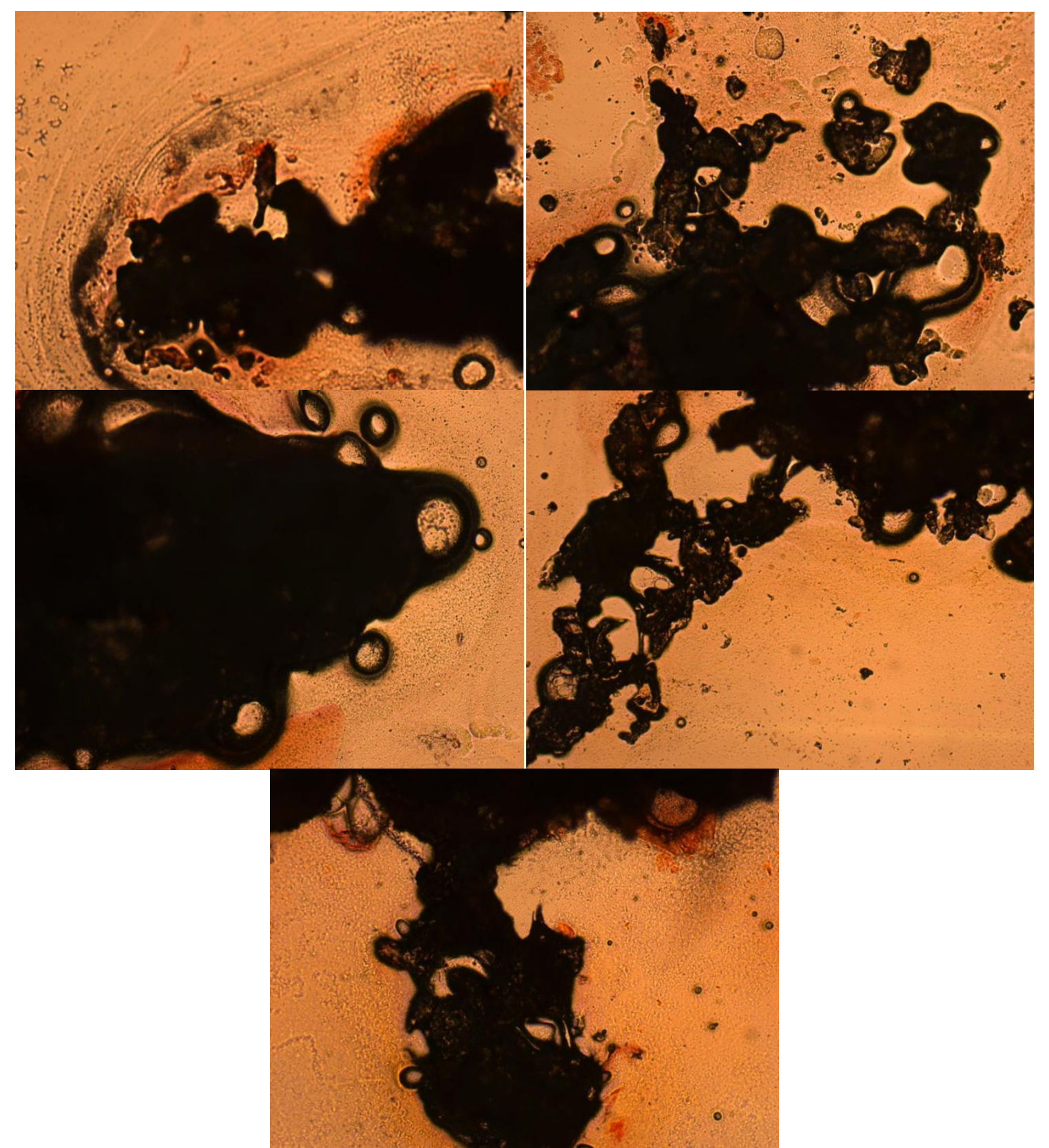

Fig C.3 Five inverted optical microscope images of Alizarin Red S-stained ODM-soaked scaffold \#1 cryosections taken at $4 \mathrm{x}$ magnification 


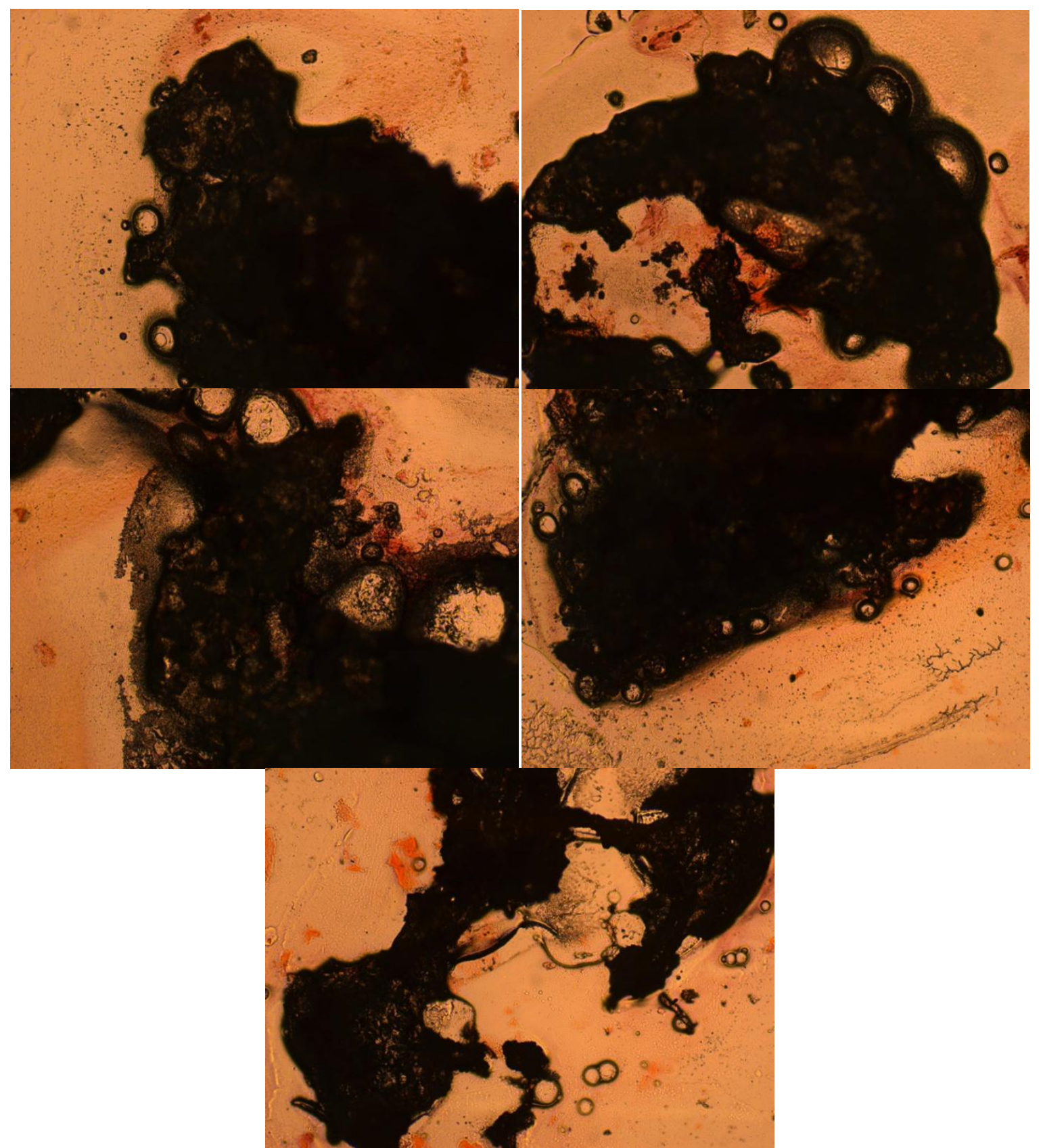

Fig C.4 Five inverted optical microscope images of Alizarin Red S-stained ODM-soaked scaffold \#2 cryosections taken at $4 \mathrm{x}$ magnification 


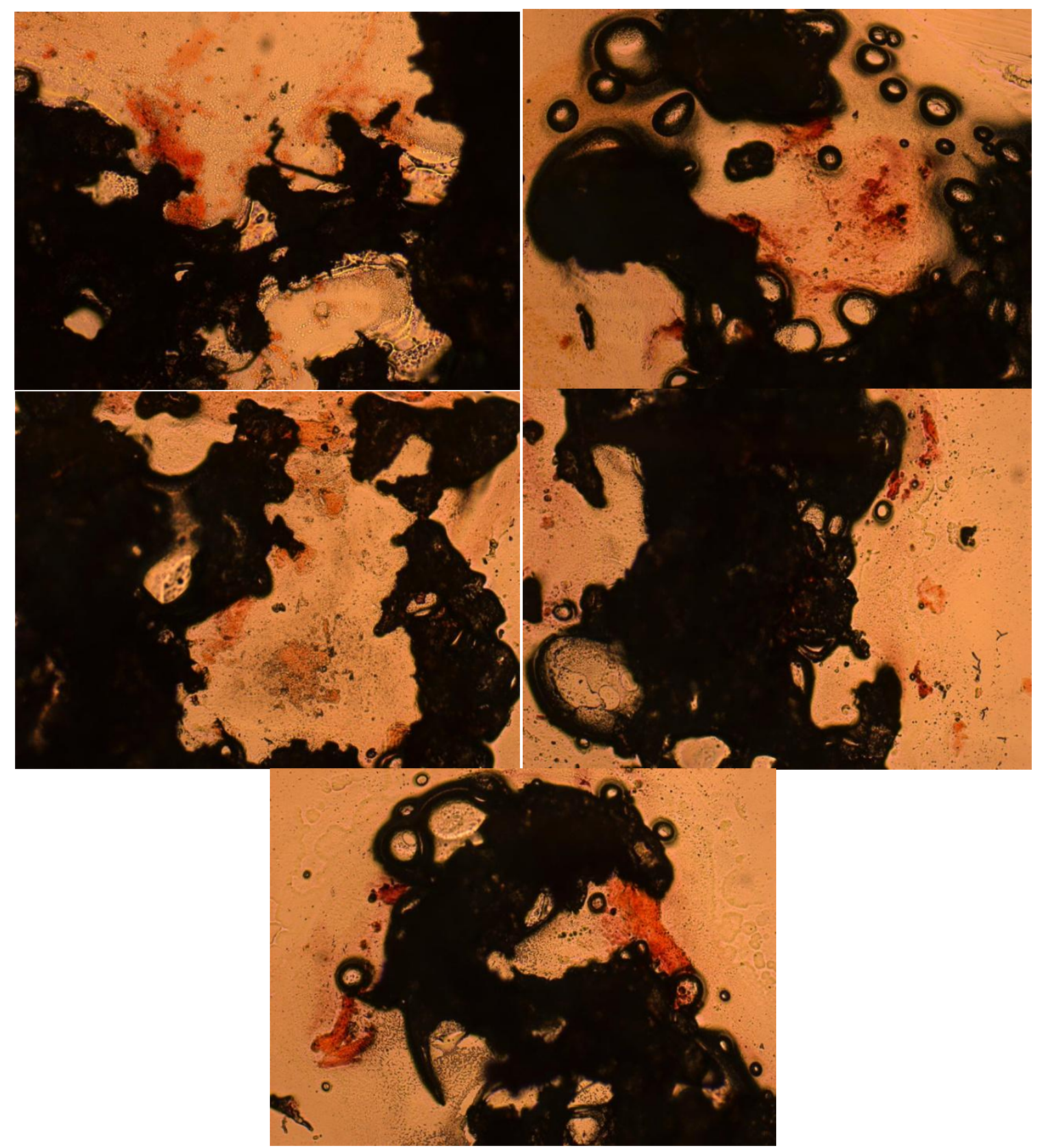

Fig C.5 Five inverted optical microscope images of Alizarin Red S-stained ODM-soaked scaffold \#3 cryosections taken at $4 \mathrm{x}$ magnification 


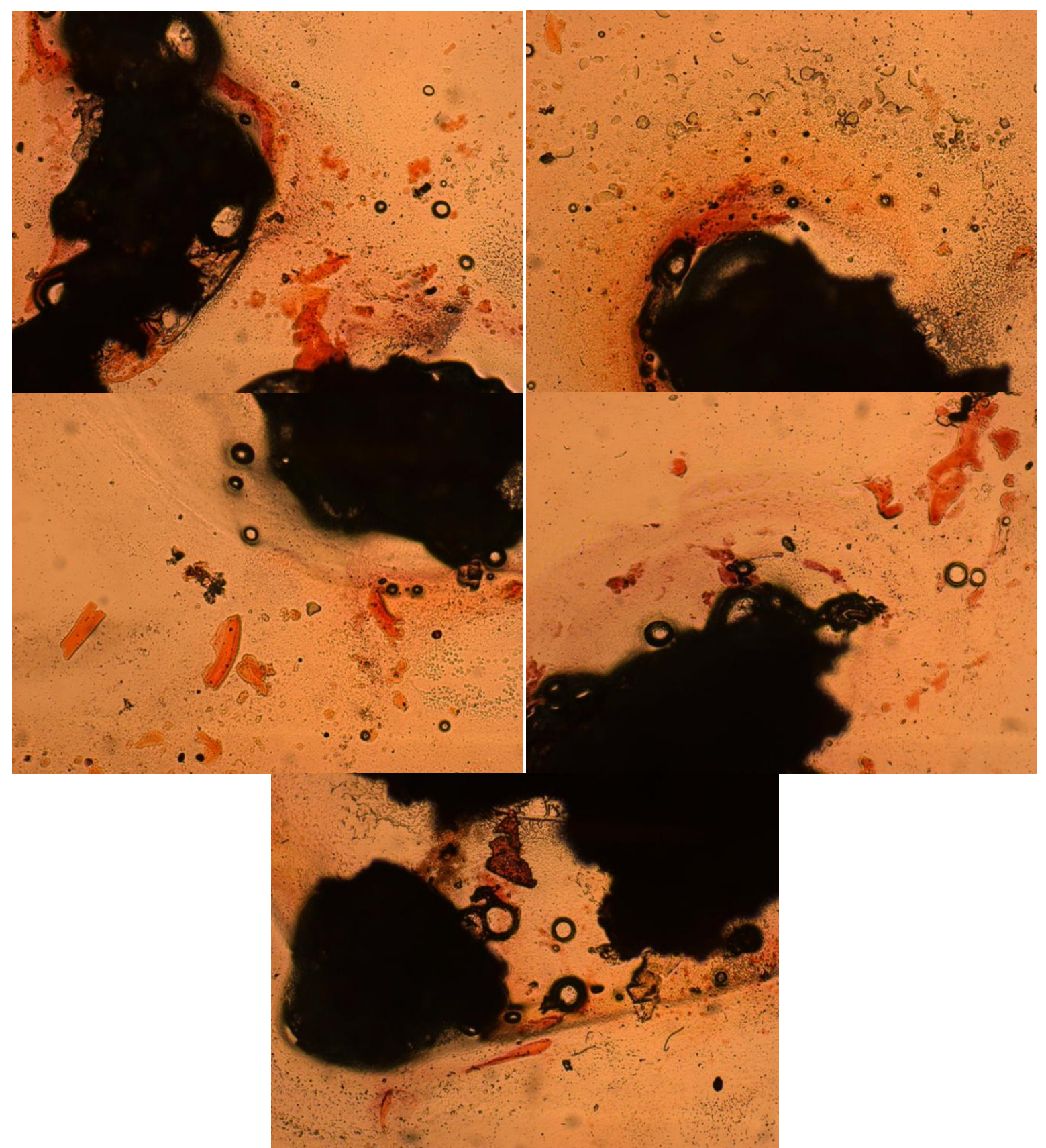

Fig C.6 Five inverted optical microscope images of Alizarin Red S-stained ODM-soaked scaffold with osteoblast action \#1 cryosections taken at $4 \mathrm{x}$ magnification 


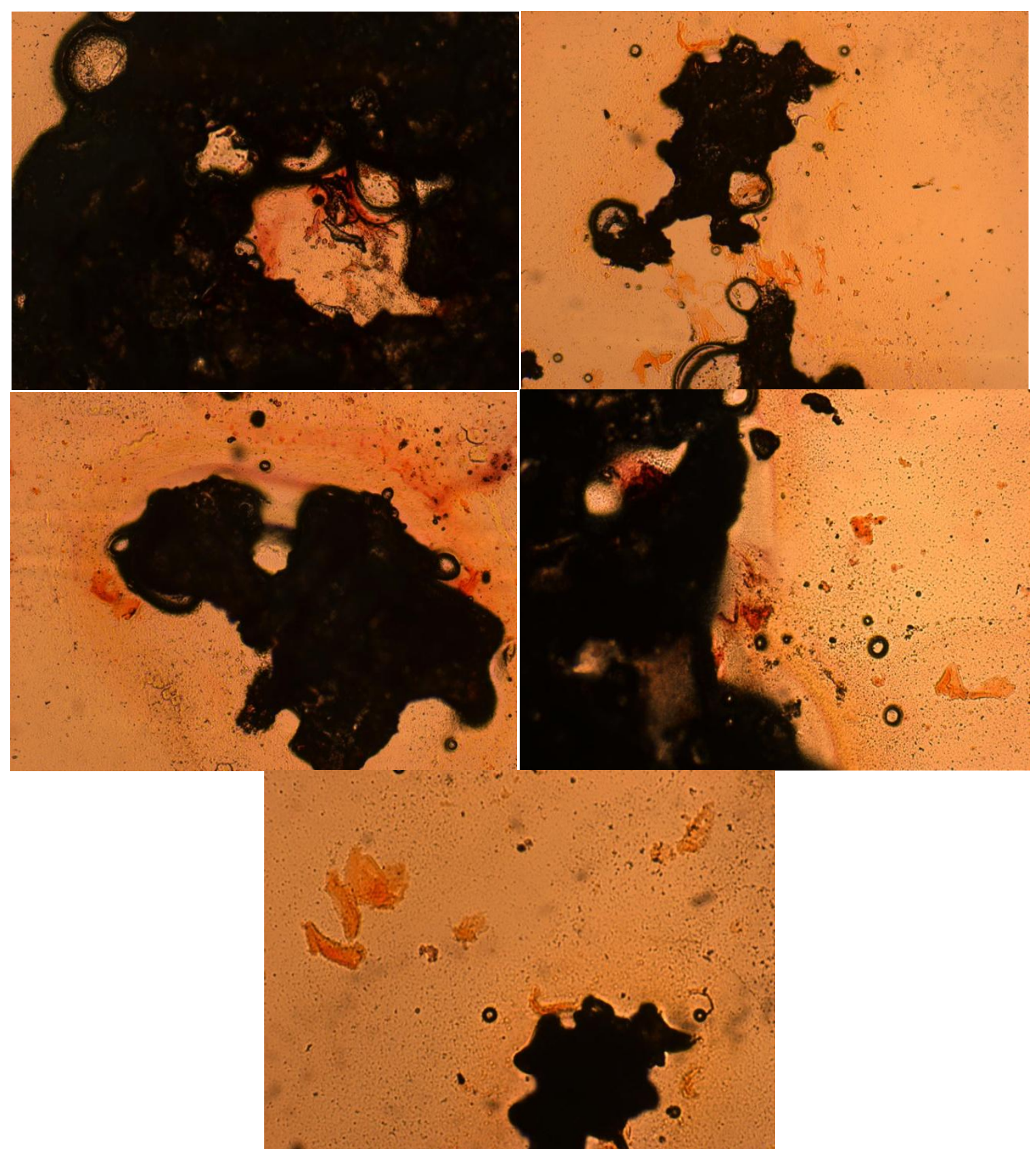

Fig C.7 Five inverted optical microscope images of Alizarin Red S-stained ODM-soaked scaffold with osteoblast action \#2 cryosections taken at $4 \mathrm{x}$ magnification 


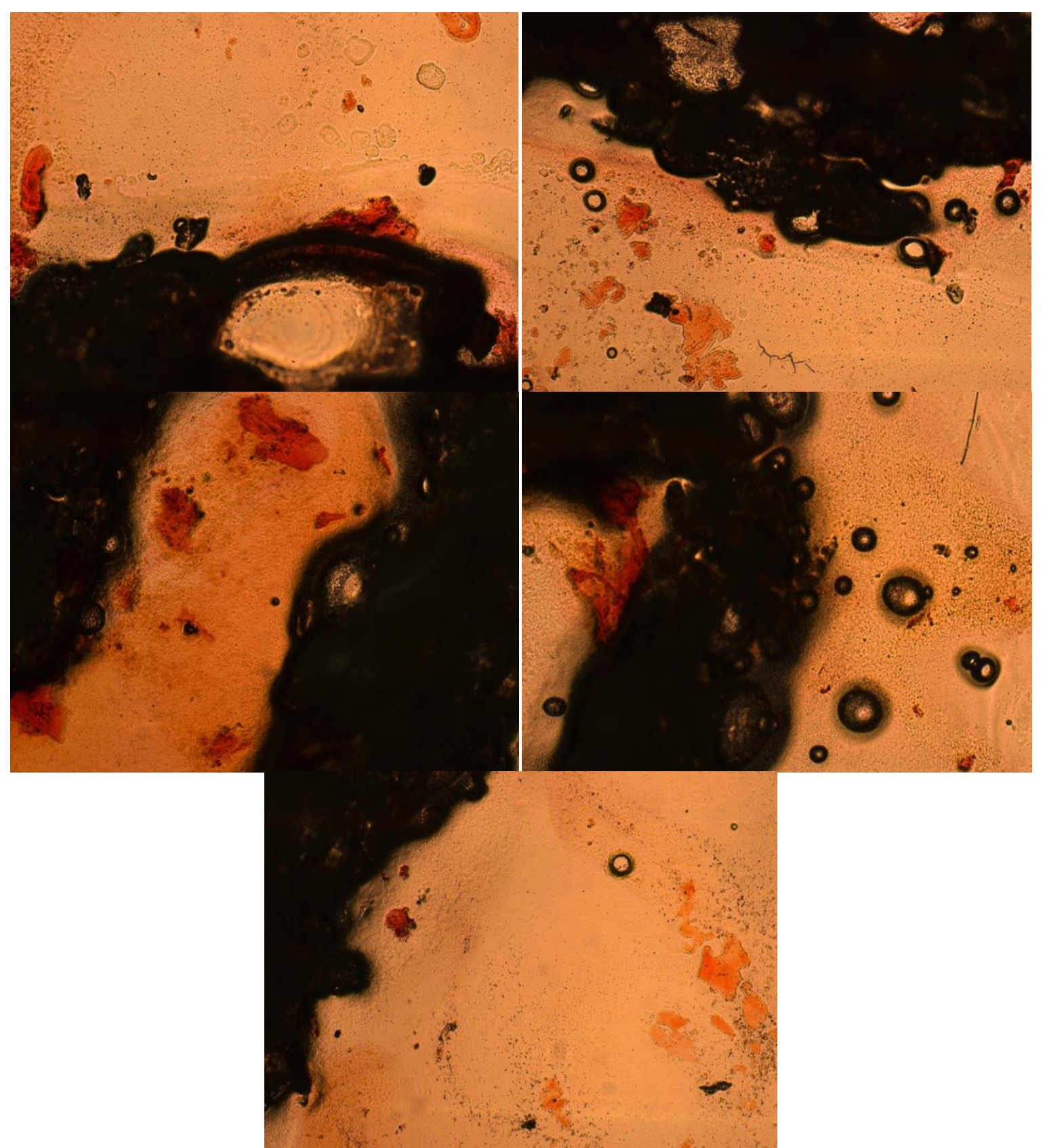

Fig C.8 Five inverted optical microscope images of Alizarin Red S-stained ODM-soaked scaffold with osteoblast action \#3 cryosections taken at $4 \mathrm{x}$ magnification 


\section{APPENDIX D: COMPRESSION TESTING RAW DATA}

Table D.1 Osteoblast-Containing Scaffolds Specimen 1 Raw Compression Data

\begin{tabular}{|c|c|c|c|c|c|c|c|}
\hline $\begin{array}{c}\begin{array}{c}\text { Extension } \\
(\mathrm{mm})\end{array} \\
\end{array}$ & $\begin{array}{l}\text { Load } \\
\text { [N] }\end{array}$ & \begin{tabular}{|c}
$\begin{array}{c}\text { Compressive stress } \\
\text { [MPa] }\end{array}$ \\
\end{tabular} & \begin{tabular}{|c|} 
Compressive strain \\
(mm/mm)
\end{tabular} & $\begin{array}{c}\text { Extension } \\
\text { (mm) }\end{array}$ & $\begin{array}{l}\text { Load } \\
\text { (N) }\end{array}$ & \begin{tabular}{|c|} 
Compressive stress \\
[MPa]
\end{tabular} & \begin{tabular}{|c|} 
Fompressive strain \\
[mm/mm]
\end{tabular} \\
\hline 0 & -0.00017 & -0.00001 & \begin{tabular}{|c|}
0 \\
\end{tabular} & 2.76031 & 0.00111 & 0.00006 & 0.55206 \\
\hline 0.00328 & -0.00035 & -0.00002 & 0.00066 & 2.80312 & 0.00235 & 0.00012 & 0.56062 \\
\hline 0.02547 & 0.01944 & 0.00099 & 0.00509 & 2.84562 & 0.00278 & 0.00014 & 0.56912 \\
\hline 0.06813 & 0.05741 & 0.00292 & 0.01363 & 2.88797 & 0.00046 & 0.00002 & 0.57759 \\
\hline 0.11375 & 0.07584 & 0.00386 & 0.02275 & 2.93047 & -0.00119 & -0.00006 & 0.58609 \\
\hline 0.155 & 0.05843 & 0.00298 & 0.031 & 2.97297 & 0.00049 & 0.00003 & 0.59459 \\
\hline 0.19797 & 0.03251 & 0.00166 & 0.03959 & 3.01516 & 0.00112 & 0.00006 & 0.60303 \\
\hline 0.24703 & 0.0211 & 0.00107 & 0.04941 & 3.05703 & -0.00063 & -0.00003 & 0.61141 \\
\hline 0.29766 & 0.01953 & 0.00099 & 0.05953 & 3.09859 & -0.00043 & -0.00002 & 0.61972 \\
\hline 0.34594 & 0.01089 & 0.00055 & 0.06919 & 3.14062 & -0.00022 & -0.00001 & 0.62813 \\
\hline 0.39281 & -0.00146 & -0.00007 & 0.07856 & 3.18297 & 0.00097 & 0.00005 & 0.63659 \\
\hline 0.43984 & -0.00708 & -0.00036 & 0.08797 & 3.22562 & 0.00164 & 0.00008 & 0.64512 \\
\hline 0.48703 & -0.00557 & -0.00028 & 0.09741 & 3.26781 & 0.00141 & 0.00007 & 0.65356 \\
\hline 0.53344 & -0.00144 & -0.00007 & 0.10669 & 3.31031 & 0.00141 & 0.00007 & 0.66206 \\
\hline 0.57844 & -0.00366 & -0.00019 & 0.11569 & 3.35281 & 0.00047 & 0.00002 & 0.67056 \\
\hline 0.62281 & -0.00449 & -0.00023 & 0.12456 & 3.39453 & 0.00049 & 0.00003 & 0.67891 \\
\hline 0.6675 & -0.00205 & -0.0001 & 0.1335 & 3.43609 & -0.00041 & -0.00002 & 0.68722 \\
\hline 0.71266 & 0.00082 & 0.00004 & 0.14253 & 3.47859 & 0.00125 & 0.00006 & 0.69572 \\
\hline 0.75719 & 0.00079 & 0.00004 & 0.15144 & 3.52203 & 0.00256 & 0.00013 & 0.70441 \\
\hline 0.80094 & -0.00052 & -0.00003 & 0.16019 & 3.56547 & 0.00238 & 0.00012 & 0.71309 \\
\hline 0.84469 & -0.00173 & -0.00009 & 0.16894 & 3.60828 & -0.00067 & -0.00003 & 0.72166 \\
\hline 0.88844 & -0.00096 & -0.00005 & 0.17769 & 3.65047 & -0.00118 & -0.00006 & 0.73009 \\
\hline 0.93172 & -0.00247 & -0.00013 & 0.18634 & 3.6925 & 0.00018 & 0.00001 & 0.7385 \\
\hline 0.97516 & -0.00322 & -0.00016 & 0.19503 & 3.73391 & 0.00042 & 0.00002 & 0.74678 \\
\hline 1.01922 & 0.00004 & 0 & 0.20384 & 3.77562 & 0.00155 & 0.00008 & 0.75512 \\
\hline 1.06297 & 0.00083 & 0.00004 & 0.21259 & 3.81781 & 0.00212 & 0.00011 & 0.76356 \\
\hline 1.10609 & -0.00185 & -0.00009 & 0.22122 & 3.86031 & 0.00064 & 0.00003 & 0.77206 \\
\hline 1.14875 & -0.00395 & -0.0002 & 0.22975 & 3.90266 & 0.00186 & 0.00009 & 0.78053 \\
\hline 1.19109 & -0.00257 & -0.00013 & 0.23822 & 3.94531 & 0.0039 & 0.0002 & 0.78906 \\
\hline 1.23328 & -0.0015 & -0.00008 & 0.24666 & 3.98781 & 0.00383 & 0.0002 & 0.79756 \\
\hline 1.27563 & -0.00079 & -0.00004 & 0.25513 & 4.03031 & 0.00192 & 0.0001 & 0.80606 \\
\hline 1.31828 & -0.00007 & 0 & 0.26366 & 4.07281 & -0.00165 & -0.00008 & 0.81456 \\
\hline 1.36094 & 0.00215 & 0.00011 & 0.27219 & 4.11547 & -0.00161 & -0.00008 & 0.82309 \\
\hline 1.40328 & 0.00182 & 0.00009 & 0.28066 & 4.15766 & -0.00245 & -0.00012 & 0.83153 \\
\hline 1.44594 & 0.0001 & 0 & 0.28919 & 4.19922 & -0.00324 & -0.00017 & 0.83984 \\
\hline 1.48891 & 0.00185 & 0.00009 & 0.29778 & 4.24141 & -0.00074 & -0.00004 & 0.84828 \\
\hline 1.53172 & 0.0052 & 0.00026 & 0.30634 & 4.28469 & 0.00088 & 0.00004 & 0.85694 \\
\hline 1.57438 & 0.00532 & 0.00027 & 0.31488 & 4.3275 & 0.00084 & 0.00004 & 0.8655 \\
\hline 1.61734 & 0.00235 & 0.00012 & 0.32347 & 4.36875 & 0.00032 & 0.00002 & 0.87375 \\
\hline 1.66031 & 0.00069 & 0.00004 & 0.33206 & 4.41047 & 0.00016 & 0.00001 & 0.88209 \\
\hline 1.70297 & 0.00146 & 0.00007 & 0.34059 & 4.45313 & 0.00284 & 0.00014 & 0.89063 \\
\hline 1.74516 & 0.00016 & 0.00001 & 0.34903 & 4.49563 & 0.00388 & 0.0002 & 0.89913 \\
\hline 1.78734 & -0.00207 & -0.00011 & 0.35747 & 4.53797 & 0.00221 & 0.00011 & 0.90759 \\
\hline 1.82922 & -0.00028 & -0.00001 & 0.36584 & 4.58063 & 0.00081 & 0.00004 & 0.91613 \\
\hline 1.87078 & -0.00142 & -0.00007 & 0.37416 & 4.62359 & 0.00042 & 0.00002 & 0.92472 \\
\hline 1.91266 & -0.00176 & -0.00009 & 0.38253 & 4.66594 & 0.00229 & 0.00012 & 0.93319 \\
\hline 1.955 & 0.00033 & 0.00002 & 0.391 & 4.70859 & 0.00151 & 0.00008 & 0.94172 \\
\hline 1.99734 & 0.00196 & 0.0001 & 0.39947 & 4.75156 & 0.00103 & 0.00005 & 0.95031 \\
\hline 2.03984 & 0.00389 & 0.0002 & 0.40797 & 4.79437 & 0.00158 & 0.00008 & 0.95888 \\
\hline 2.08266 & 0.00375 & 0.00019 & 0.41653 & 4.83672 & 0.00035 & 0.00002 & 0.96734 \\
\hline 2.12563 & 0.00289 & 0.00015 & 0.42513 & 4.87828 & -0.00215 & -0.00011 & 0.97566 \\
\hline 2.16828 & 0.00246 & 0.00013 & 0.43366 & 4.91984 & -0.00101 & -0.00005 & 0.98397 \\
\hline 2.21078 & 0.00184 & 0.00009 & 0.44216 & 4.96156 & 0.00178 & 0.00009 & 0.99231 \\
\hline 2.25359 & 0.00069 & 0.00004 & 0.45072 & 5.00359 & 0.00203 & 0.0001 & 1.00072 \\
\hline 2.29641 & 0.0005 & 0.00003 & 0.45928 & 5.04625 & 0.00187 & 0.0001 & 1.00925 \\
\hline 2.33875 & -0.00142 & -0.00007 & 0.46775 & 5.08875 & 0.00445 & 0.00023 & 1.01775 \\
\hline 2.38078 & -0.00215 & -0.00011 & 0.47616 & 5.13094 & 0.00535 & 0.00027 & 1.02619 \\
\hline 2.42297 & -0.00241 & -0.00012 & 0.48459 & 5.17312 & 0.00423 & 0.00022 & 1.03462 \\
\hline 2.46469 & -0.00165 & -0.00008 & 0.49294 & 5.21578 & 0.00318 & 0.00016 & 1.04316 \\
\hline 2.50625 & -0.00021 & -0.00001 & 0.50125 & 5.25844 & 0.00257 & 0.00013 & 1.05169 \\
\hline 2.54828 & 0.00093 & 0.00005 & 0.50966 & 5.30094 & 0.00108 & 0.00005 & 1.06019 \\
\hline 2.59078 & 0.00046 & 0.00002 & 0.51816 & 5.34312 & -0.00208 & -0.00011 & 1.06862 \\
\hline 2.63312 & -0.00022 & -0.00001 & 0.52662 & 5.38562 & -0.00156 & -0.00008 & 1.07712 \\
\hline 2.675 & 0.00074 & 0.00004 & 0.535 & 5.42859 & 0.0017 & 0.00009 & 1.08572 \\
\hline 2.71734 & 0.00032 & 0.00002 & 0.54347 & 5.47141 & 0.00107 & 0.00005 & 1.09428 \\
\hline
\end{tabular}




\begin{tabular}{|c|c|c|c|}
\hline $\begin{array}{c}\text { Extension } \\
\text { (mm) }\end{array}$ & $\begin{array}{c}\text { Load } \\
\text { (N) }\end{array}$ & $\begin{array}{c}\text { Compressive stress } \\
\text { (MPa] }\end{array}$ & $\begin{array}{c}\text { Fompressive strain } \\
\text { [mmimm] }\end{array}$ \\
\hline 5.51375 & -0.00053 & -0.00003 & 1.10275 \\
\hline 5.55547 & -0.00189 & -0.0001 & 1.11109 \\
\hline 5.59688 & -0.00173 & -0.00009 & 1.11937 \\
\hline 5.63812 & -0.00067 & -0.00003 & 1.12762 \\
\hline 5.68031 & 0.00147 & 0.00007 & 1.13606 \\
\hline 5.72281 & 0.00365 & 0.00019 & 1.14456 \\
\hline 5.76562 & 0.0022 & 0.00011 & 1.15313 \\
\hline 5.80828 & 0.00249 & 0.00013 & 1.16166 \\
\hline 5.85078 & 0.00289 & 0.00015 & 1.17016 \\
\hline 5.89328 & 0.00311 & 0.00016 & 1.17866 \\
\hline 5.93578 & 0.00367 & 0.00019 & 1.18716 \\
\hline 5.97844 & 0.00137 & 0.00007 & 1.19569 \\
\hline 6.02109 & -0.00087 & -0.00004 & 1.20422 \\
\hline 6.06344 & -0.0018 & -0.00009 & 1.21269 \\
\hline 6.10578 & -0.00048 & -0.00002 & 1.22116 \\
\hline 6.14813 & -0.00184 & -0.00009 & 1.22963 \\
\hline 6.19031 & -0.00181 & -0.00009 & 1.23806 \\
\hline 6.23203 & -0.00124 & -0.00006 & 1.24641 \\
\hline 6.27391 & -0.0029 & -0.00015 & 1.25478 \\
\hline 6.31625 & -0.00227 & -0.00012 & 1.26325 \\
\hline 6.35859 & 0.00105 & 0.00005 & 1.27172 \\
\hline 6.40078 & 0.00379 & 0.00019 & 1.28016 \\
\hline 6.44344 & 0.00445 & 0.00023 & 1.28869 \\
\hline 6.48562 & 0.00341 & 0.00017 & 1.29712 \\
\hline 6.5275 & 0.00161 & 0.00008 & 1.3055 \\
\hline 6.57 & 0.00309 & 0.00016 & 1.314 \\
\hline 6.61297 & 0.00288 & 0.00015 & 1.32259 \\
\hline 6.65594 & 0.0046 & 0.00023 & 1.33119 \\
\hline 6.69828 & 0.00437 & 0.00022 & 1.33966 \\
\hline 6.74062 & 0.00195 & 0.0001 & 1.34812 \\
\hline 6.78281 & 0.00078 & 0.00004 & 1.35656 \\
\hline 6.82453 & 0.00117 & 0.00006 & 1.36491 \\
\hline 6.86609 & 0.00367 & 0.00019 & 1.37322 \\
\hline 6.90813 & 0.00328 & 0.00017 & 1.38163 \\
\hline 6.95047 & 0.00285 & 0.00015 & 1.39009 \\
\hline 6.99297 & 0.0032 & 0.00016 & 1.39859 \\
\hline 7.03547 & 0.00352 & 0.00018 & 1.40709 \\
\hline 7.07828 & 0.00194 & 0.0001 & 1.41566 \\
\hline 7.12094 & 0.00286 & 0.00015 & 1.42419 \\
\hline 7.16359 & 0.00344 & 0.00017 & 1.43272 \\
\hline 7.20609 & 0.00229 & 0.00012 & 1.44122 \\
\hline 7.24875 & 0.00274 & 0.00014 & 1.44975 \\
\hline 7.29141 & 0.00264 & 0.00013 & 1.45828 \\
\hline 7.33375 & 0.00401 & 0.0002 & 1.46675 \\
\hline 7.37609 & 0.00379 & 0.00019 & 1.47522 \\
\hline 7.41859 & 0.00313 & 0.00016 & 1.48372 \\
\hline 7.46047 & 0.00005 & 0 & 1.49209 \\
\hline 7.50203 & -0.0008 & -0.00004 & 1.50041 \\
\hline 7.54406 & 0.00169 & 0.00009 & 1.50881 \\
\hline 7.58641 & 0.00306 & 0.00016 & 1.51728 \\
\hline 7.62891 & 0.00253 & 0.00013 & 1.52578 \\
\hline 7.67125 & 0.00213 & 0.00011 & 1.53425 \\
\hline 7.71312 & 0.00122 & 0.00006 & 1.54262 \\
\hline 7.75516 & -0.00049 & -0.00002 & 1.55103 \\
\hline 7.7975 & 0.00023 & 0.00001 & 1.5595 \\
\hline 7.84016 & 0.00295 & 0.00015 & 1.56803 \\
\hline 7.88328 & 0.00441 & 0.00022 & 1.57666 \\
\hline 7.92594 & 0.00149 & 0.00008 & 1.58519 \\
\hline 7.96812 & 0.00071 & 0.00004 & 1.59362 \\
\hline 8.01062 & 0.0027 & 0.00014 & 1.60213 \\
\hline 8.05281 & 0.00235 & 0.00012 & 1.61056 \\
\hline 8.09422 & 0.00062 & 0.00003 & 1.61884 \\
\hline 8.13562 & 0.0015 & 0.00008 & 1.62713 \\
\hline 8.17781 & 0.00189 & 0.0001 & 1.63556 \\
\hline 8.22031 & 0.00263 & 0.00013 & 1.64406 \\
\hline
\end{tabular}

\begin{tabular}{|c|c|c|c|}
\hline $\begin{array}{c}\text { Extension } \\
\text { (mm) }\end{array}$ & $\begin{array}{c}\text { Load } \\
\text { (N) }\end{array}$ & $\begin{array}{c}\text { Compressive stress } \\
\text { (MPa] }\end{array}$ & $\begin{array}{c}\begin{array}{c}\text { fompressive strain } \\
\text { [mmimm }]\end{array} \\
\end{array}$ \\
\hline 8.2625 & 0.00362 & 0.00018 & 1.6525 \\
\hline 8.30516 & 0.00345 & 0.00018 & 1.66103 \\
\hline 8.34797 & 0.00491 & 0.00025 & 1.66959 \\
\hline 8.39062 & 0.00371 & 0.00019 & 1.67813 \\
\hline 8.43281 & 0.00081 & 0.00004 & 1.68656 \\
\hline 8.47516 & 0.00061 & 0.00003 & 1.69503 \\
\hline 8.51766 & 0.00247 & 0.00013 & 1.70353 \\
\hline 8.56 & 0.00344 & 0.00018 & 1.712 \\
\hline 8.6025 & 0.00399 & 0.0002 & 1.7205 \\
\hline 8.64516 & 0.00383 & 0.0002 & 1.72903 \\
\hline 8.68734 & 0.00119 & 0.00006 & 1.73747 \\
\hline 8.72891 & -0.00172 & -0.00009 & 1.74578 \\
\hline 8.77063 & -0.00125 & -0.00006 & 1.75412 \\
\hline 8.81281 & 0.00238 & 0.00012 & 1.76256 \\
\hline 8.85531 & 0.0037 & 0.00019 & 1.77106 \\
\hline 8.8975 & 0.00103 & 0.00005 & 1.7795 \\
\hline 8.93984 & 0.00017 & 0.00001 & 1.78797 \\
\hline 8.9825 & 0.00138 & 0.00007 & 1.7965 \\
\hline 9.02531 & 0.00228 & 0.00012 & 1.80506 \\
\hline 9.06781 & 0.00272 & 0.00014 & 1.81356 \\
\hline 9.11016 & 0.0041 & 0.00021 & 1.82203 \\
\hline 9.15281 & 0.00448 & 0.00023 & 1.83056 \\
\hline 9.19531 & 0.00302 & 0.00015 & 1.83906 \\
\hline 9.23797 & 0.00251 & 0.00013 & 1.84759 \\
\hline 9.28047 & 0.00426 & 0.00022 & 1.85609 \\
\hline 9.3225 & 0.00293 & 0.00015 & 1.8645 \\
\hline 9.36391 & -0.00043 & -0.00002 & 1.87278 \\
\hline 9.40578 & 0.00075 & 0.00004 & 1.88116 \\
\hline 9.44812 & 0.00214 & 0.00011 & 1.88962 \\
\hline 9.49078 & 0.00345 & 0.00018 & 1.89816 \\
\hline 9.53312 & 0.00259 & 0.00013 & 1.90663 \\
\hline 9.57563 & 0.00231 & 0.00012 & 1.91513 \\
\hline 9.61828 & 0.00287 & 0.00015 & 1.92366 \\
\hline 9.66078 & 0.00376 & 0.00019 & 1.93216 \\
\hline 9.70328 & 0.00398 & 0.0002 & 1.94066 \\
\hline 9.74609 & 0.02032 & 0.00103 & 1.94922 \\
\hline 9.78891 & 0.095 & 0.00484 & 1.95778 \\
\hline 9.83141 & 0.24829 & 0.01265 & 1.96628 \\
\hline 9.87359 & 0.48745 & 0.02483 & 1.97472 \\
\hline 9.91547 & 0.81143 & 0.04133 & 1.98309 \\
\hline 9.95719 & 1.21765 & 0.06201 & 1.99144 \\
\hline 9.99875 & 1.68917 & 0.08603 & 1.99975 \\
\hline 10.04078 & 2.22444 & 0.11329 & 2.00816 \\
\hline 10.08313 & 2.81215 & 0.14322 & 2.01663 \\
\hline 10.12547 & 3.44155 & 0.17528 & 2.02509 \\
\hline 10.16797 & 4.09746 & 0.20868 & 2.03359 \\
\hline 10.21047 & 4.77197 & 0.24303 & 2.04209 \\
\hline 10.25328 & 5.46796 & 0.27848 & 2.05066 \\
\hline 10.29594 & 6.1868 & 0.31509 & 2.05919 \\
\hline 10.33844 & 6.932 & 0.35304 & 2.06769 \\
\hline 10.38141 & 7.70794 & 0.39256 & 2.07628 \\
\hline 10.42422 & 8.48794 & 0.43229 & 2.08484 \\
\hline 10.46656 & 9.10772 & 0.46385 & 2.09331 \\
\hline 10.50891 & 9.50492 & 0.48408 & 2.10178 \\
\hline 10.55078 & 9.77937 & 0.49806 & 2.11016 \\
\hline 10.59219 & 10.01594 & 0.51011 & 2.11844 \\
\hline 10.63359 & 10.36294 & 0.52778 & 2.12672 \\
\hline 10.67578 & 11.00829 & 0.56065 & 2.13516 \\
\hline 10.71813 & 11.88596 & 0.60535 & 2.14363 \\
\hline 10.76031 & 12.90906 & 0.65745 & 2.15206 \\
\hline 10.80281 & 14.02159 & 0.71411 & 2.16056 \\
\hline 10.84562 & 15.195 & 0.77387 & 2.16913 \\
\hline 10.88828 & 16.42106 & 0.83632 & 2.17766 \\
\hline 10.93062 & 17.6923 & 0.90106 & 2.18613 \\
\hline 10.97312 & 19.00143 & 0.96774 & 2.19462 \\
\hline
\end{tabular}




\begin{tabular}{|c|c|c|c|}
\hline $\begin{array}{l}\text { Extension } \\
\text { (mm) }\end{array}$ & $\begin{array}{c}\text { Load } \\
\text { (N) }\end{array}$ & $\begin{array}{c}\text { Compressive stress } \\
\text { (MPa) }\end{array}$ & \begin{tabular}{c|} 
fompressive strain \\
[mm/mm
\end{tabular} \\
\hline 11.01594 & 20.35987 & 1.03692 & 2.20319 \\
\hline 11.05828 & 21.77502 & 1.10899 & 2.21166 \\
\hline 11.10031 & 23.2463 & 1.18392 & 2.22006 \\
\hline 11.14234 & 24.78502 & 1.26229 & 2.22847 \\
\hline 11.18438 & 26.40514 & 1.3448 & 2.23688 \\
\hline 11.22625 & 28.12587 & 1.43244 & 2.24525 \\
\hline 11.26812 & 29.93759 & 1.52471 & 2.25362 \\
\hline 11.31078 & 31.84958 & 1.62209 & 2.26216 \\
\hline 11.35344 & 33.8854 & 1.72577 & 2.27069 \\
\hline 11.39531 & 36.04839 & 1.83593 & 2.27906 \\
\hline 11.43766 & 38.33357 & 1.95231 & 2.28753 \\
\hline 11.48047 & 40.76493 & 2.07614 & 2.29609 \\
\hline 11.52281 & 43.36512 & 2.20857 & 2.30456 \\
\hline 11.56516 & 46.11535 & 2.34864 & 2.31303 \\
\hline 11.60781 & 49.02795 & 2.49697 & 2.32156 \\
\hline 11.65031 & 52.12078 & 2.65449 & 2.33006 \\
\hline 11.69281 & 55.39218 & 2.8211 & 2.33856 \\
\hline 11.73516 & 58.85316 & 2.99737 & 2.34703 \\
\hline 11.77766 & 62.53292 & 3.18478 & 2.35553 \\
\hline 11.81938 & 66.45048 & 3.38429 & 2.36388 \\
\hline 11.86063 & 70.60126 & 3.59569 & 2.37213 \\
\hline 11.9025 & 74.98656 & 3.81903 & 2.3805 \\
\hline 11.94484 & 79.66535 & 4.05732 & 2.38897 \\
\hline 11.98734 & 84.67841 & 4.31264 & 2.39747 \\
\hline 12.02953 & 90.02114 & 4.58474 & 2.40591 \\
\hline 12.07203 & 95.68906 & 4.8734 & 2.41441 \\
\hline 12.11484 & 101.69812 & 5.17944 & 2.42297 \\
\hline 12.1575 & 108.12687 & 5.50686 & 2.4315 \\
\hline 12.19984 & 114.99017 & 5.8564 & 2.43997 \\
\hline 12.24234 & 122.24084 & 6.22567 & 2.44847 \\
\hline 12.285 & 129.96597 & 6.61911 & 2.457 \\
\hline 12.32734 & 138.22557 & 7.03977 & 2.46547 \\
\hline 12.36953 & 147.00972 & 7.48714 & 2.47391 \\
\hline 12.41172 & 156.34169 & 7.96242 & 2.48234 \\
\hline 12.45344 & 166.32115 & 8.47067 & 2.49069 \\
\hline 12.49469 & 176.95869 & 9.01243 & 2.49894 \\
\hline 12.53625 & 188.21748 & 9.58584 & 2.50725 \\
\hline 12.57844 & 200.23936 & 10.19811 & 2.51569 \\
\hline 12.62031 & 213.16719 & 10.85652 & 2.52406 \\
\hline 12.66234 & 226.96295 & 11.55913 & 2.53247 \\
\hline 12.70469 & 241.60651 & 12.30492 & 2.54094 \\
\hline 12.74719 & 257.14737 & 13.09641 & 2.54944 \\
\hline 12.78953 & 273.63669 & 13.9362 & 2.55791 \\
\hline 12.83187 & 291.07312 & 14.82423 & 2.56638 \\
\hline 12.87453 & 309.50183 & 15.7628 & 2.57491 \\
\hline 12.91703 & 329.08002 & 16.75991 & 2.58341 \\
\hline 12.95922 & 349.8804 & 17.81926 & 2.59184 \\
\hline 13.00094 & 371.974 & 18.94448 & 2.60019 \\
\hline 13.04266 & 395.35361 & 20.13519 & 2.60853 \\
\hline 13.08406 & 420.07065 & 21.39402 & 2.61681 \\
\hline 13.12531 & 446.3819 & 22.73404 & 2.62506 \\
\hline 13.13344 & 451.85394 & 23.01273 & 2.62669 \\
\hline
\end{tabular}


Table D.2 Osteoblast-Containing Scaffolds Specimen 2 Raw Compression Data

\begin{tabular}{|c|c|c|c|c|c|c|c|}
\hline $\begin{array}{c}\text { Extension } \\
\text { (mm) }\end{array}$ & $\begin{array}{l}\text { Load } \\
\text { [N] }\end{array}$ & \begin{tabular}{|c|}
$\begin{array}{c}\text { Compressive stress } \\
\text { (MPa) }\end{array}$ \\
\end{tabular} & $\begin{array}{c}\text { fompressive strain } \\
\text { [mmlmm] }\end{array}$ & $\begin{array}{c}\begin{array}{c}\text { Extension } \\
\text { (mm) }\end{array} \\
\end{array}$ & $\begin{array}{l}\text { Load } \\
\text { (N) }\end{array}$ & \begin{tabular}{|c} 
Compressive stress \\
(MPa)
\end{tabular} & $\begin{array}{c}\text { Compressive strain } \\
{[\mathrm{mm} / \mathrm{mm}]}\end{array}$ \\
\hline 0 & 0.00372 & \begin{tabular}{|c|}
0.00019 \\
\end{tabular} & \begin{tabular}{|c|}
0 \\
\end{tabular} & 2.76016 & 0.00603 & 0.00031 & 0.55203 \\
\hline 0.00281 & 0.00382 & 0.00019 & 0.00056 & $\begin{array}{l}\text { 2.r6016 } \\
2.80266\end{array}$ & 0.00584 & 0.0003 & 0.56053 \\
\hline 0.02359 & 0.02236 & 0.00114 & 0.00472 & 2.84516 & 0.00892 & 0.00045 & 0.56903 \\
\hline 0.06547 & 0.05898 & 0.003 & 0.01309 & 2.8875 & 0.00391 & 0.0002 & 0.5775 \\
\hline 0.11172 & 0.07989 & 0.00407 & 0.02234 & 2.93 & 0.0027 & 0.00014 & 0.586 \\
\hline 0.15313 & 0.06394 & 0.00326 & 0.03063 & 2.97234 & 0.00692 & 0.00035 & 0.59447 \\
\hline 0.19594 & 0.03654 & 0.00186 & 0.03919 & 3.01484 & 0.00675 & 0.00034 & 0.60297 \\
\hline 0.24469 & 0.02647 & 0.00135 & 0.04894 & 3.05672 & 0.00361 & 0.00018 & 0.61134 \\
\hline 0.29531 & 0.02496 & 0.00127 & 0.05906 & 3.09797 & 0.00273 & 0.00014 & 0.61959 \\
\hline 0.34406 & 0.01687 & 0.00086 & 0.06881 & 3.13953 & 0.00342 & 0.00017 & 0.62791 \\
\hline 0.39109 & 0.00519 & 0.00026 & 0.07822 & 3.18187 & 0.00699 & 0.00036 & 0.63638 \\
\hline 0.43828 & -0.0027 & -0.00014 & 0.08766 & 3.22422 & 0.00917 & 0.00047 & 0.64484 \\
\hline 0.48547 & -0.00127 & -0.00006 & 0.09709 & 3.26672 & 0.01022 & 0.00052 & 0.65334 \\
\hline 0.53188 & 0.00185 & 0.00009 & 0.10638 & 3.30922 & 0.00773 & 0.00039 & 0.66184 \\
\hline 0.57719 & -0.00167 & -0.00009 & 0.11544 & 3.35203 & 0.00575 & 0.00029 & 0.67041 \\
\hline 0.62156 & -0.00382 & -0.00019 & 0.12431 & 3.39453 & 0.00495 & 0.00025 & 0.67891 \\
\hline 0.66625 & -0.00072 & -0.00004 & 0.13325 & 3.43672 & 0.0025 & 0.00013 & 0.68734 \\
\hline 0.71141 & 0.00231 & 0.00012 & 0.14228 & 3.47906 & 0.00281 & 0.00014 & 0.69581 \\
\hline 0.75594 & 0.00394 & 0.0002 & 0.15119 & 3.52203 & 0.00484 & 0.00025 & 0.70441 \\
\hline 0.8 & 0.00341 & 0.00017 & 0.16 & 3.56484 & 0.00531 & 0.00027 & 0.71297 \\
\hline 0.84391 & 0.0054 & 0.00028 & 0.16878 & 3.60719 & 0.00349 & 0.00018 & 0.72144 \\
\hline 0.88766 & 0.0056 & 0.00029 & 0.17753 & 3.64922 & 0.00298 & 0.00015 & 0.72984 \\
\hline 0.93109 & 0.00212 & 0.00011 & 0.18622 & 3.69125 & 0.00496 & 0.00025 & 0.73825 \\
\hline 0.97469 & 0.00363 & 0.00018 & 0.19494 & 3.73312 & 0.00481 & 0.00024 & 0.74663 \\
\hline 1.01844 & 0.00392 & 0.0002 & 0.20369 & 3.77531 & 0.00405 & 0.00021 & 0.75506 \\
\hline 1.06219 & 0.00246 & 0.00013 & 0.21244 & 3.8175 & 0.00568 & 0.00029 & 0.7635 \\
\hline 1.10563 & 0.00205 & 0.0001 & 0.22113 & 3.85969 & 0.00546 & 0.00028 & 0.77194 \\
\hline 1.14828 & 0.00079 & 0.00004 & 0.22966 & 3.90188 & 0.00747 & 0.00038 & 0.78038 \\
\hline 1.19062 & -0.00013 & -0.00001 & 0.23812 & 3.94453 & 0.01054 & 0.00054 & 0.78891 \\
\hline 1.23266 & 0.00143 & 0.00007 & 0.24653 & 3.98703 & 0.01146 & 0.00058 & 0.79741 \\
\hline 1.275 & 0.00369 & 0.00019 & 0.255 & 4.02938 & 0.00864 & 0.00044 & 0.80588 \\
\hline 1.31766 & 0.00682 & 0.00035 & 0.26353 & 4.07172 & 0.00541 & 0.00028 & 0.81434 \\
\hline 1.36047 & 0.00775 & 0.00039 & 0.27209 & 4.11469 & 0.00746 & 0.00038 & 0.82294 \\
\hline 1.40266 & 0.00624 & 0.00032 & 0.28053 & 4.15781 & 0.00848 & 0.00043 & 0.83156 \\
\hline 1.44531 & 0.00454 & 0.00023 & 0.28906 & 4.20016 & 0.00652 & 0.00033 & 0.84003 \\
\hline 1.48797 & 0.00632 & 0.00032 & 0.29759 & 4.24234 & 0.00702 & 0.00036 & 0.84847 \\
\hline 1.53063 & 0.0075 & 0.00038 & 0.30613 & 4.28469 & 0.00673 & 0.00034 & 0.85694 \\
\hline 1.57313 & 0.00675 & 0.00034 & 0.31462 & 4.32687 & 0.00307 & 0.00016 & 0.86537 \\
\hline 1.61609 & 0.00698 & 0.00036 & 0.32322 & 4.36812 & 0.00298 & 0.00015 & 0.87363 \\
\hline 1.65922 & 0.00529 & 0.00027 & 0.33184 & 4.40984 & 0.00585 & 0.0003 & 0.88197 \\
\hline 1.70203 & 0.00599 & 0.0003 & 0.34041 & 4.45219 & 0.00782 & 0.0004 & 0.89044 \\
\hline 1.74453 & 0.00666 & 0.00034 & 0.34891 & 4.49453 & 0.00827 & 0.00042 & 0.89891 \\
\hline 1.78672 & 0.00602 & 0.00031 & 0.35734 & 4.53703 & 0.00991 & 0.0005 & 0.90741 \\
\hline 1.82859 & 0.00593 & 0.0003 & 0.36572 & 4.57953 & 0.00813 & 0.00041 & 0.91591 \\
\hline 1.87047 & 0.00353 & 0.00018 & 0.37409 & 4.62234 & 0.00696 & 0.00035 & 0.92447 \\
\hline 1.91234 & 0.00409 & 0.00021 & 0.38247 & 4.66469 & 0.00996 & 0.00051 & 0.93294 \\
\hline 1.95469 & 0.00573 & 0.00029 & 0.39094 & 4.70719 & 0.00724 & 0.00037 & 0.94144 \\
\hline 1.99719 & 0.00737 & 0.00038 & 0.39944 & 4.75016 & 0.00476 & 0.00024 & 0.95003 \\
\hline 2.03969 & 0.01015 & 0.00052 & 0.40794 & 4.79266 & 0.00603 & 0.00031 & 0.95853 \\
\hline 2.08234 & 0.00744 & 0.00038 & 0.41647 & 4.835 & 0.00526 & 0.00027 & 0.967 \\
\hline 2.12516 & 0.00528 & 0.00027 & 0.42503 & 4.87812 & 0.00601 & 0.00031 & 0.97562 \\
\hline 2.16766 & 0.00731 & 0.00037 & 0.43353 & 4.92063 & 0.00863 & 0.00044 & 0.98413 \\
\hline 2.21031 & 0.00534 & 0.00027 & 0.44206 & 4.96203 & 0.00856 & 0.00044 & 0.99241 \\
\hline 2.25313 & 0.0067 & 0.00034 & 0.45063 & 5.00328 & 0.00531 & 0.00027 & 1.00066 \\
\hline 2.29578 & 0.00658 & 0.00033 & 0.45916 & 5.04547 & 0.00515 & 0.00026 & 1.00909 \\
\hline 2.33781 & 0.00376 & 0.00019 & 0.46756 & 5.08812 & 0.0093 & 0.00047 & 1.01762 \\
\hline 2.37984 & 0.00551 & 0.00028 & 0.47597 & 5.13062 & 0.01101 & 0.00056 & 1.02612 \\
\hline 2.42203 & 0.00348 & 0.00018 & 0.48441 & 5.17266 & 0.00925 & 0.00047 & 1.03453 \\
\hline 2.46406 & 0.00294 & 0.00015 & 0.49281 & 5.215 & 0.0085 & 0.00043 & 1.043 \\
\hline 2.50547 & 0.00648 & 0.00033 & 0.50109 & 5.25781 & 0.00831 & 0.00042 & 1.05156 \\
\hline 2.54734 & 0.00673 & 0.00034 & 0.50947 & 5.30047 & 0.0067 & 0.00034 & 1.06009 \\
\hline 2.59 & 0.00558 & 0.00028 & 0.518 & 5.34281 & 0.00421 & 0.00021 & 1.06856 \\
\hline 2.6325 & 0.00655 & 0.00033 & 0.5265 & 5.38562 & 0.00478 & 0.00024 & 1.07712 \\
\hline 2.675 & 0.00932 & 0.00047 & 0.535 & 5.42797 & 0.0061 & 0.00031 & 1.08559 \\
\hline 2.71766 & 0.01015 & 0.00052 & 0.54353 & 5.47016 & 0.00516 & 0.00026 & 1.09403 \\
\hline
\end{tabular}




\begin{tabular}{|c|c|c|c|}
\hline $\begin{array}{l}\text { Extension } \\
\text { (mm) }\end{array}$ & $\begin{array}{l}\text { Load } \\
\text { (N) }\end{array}$ & $\begin{array}{c}\text { Compressive stress } \\
\text { (MPa) }\end{array}$ & $\begin{array}{c}\text { Compressive strain } \\
\text { [mmimm }\end{array}$ \\
\hline 5.51234 & 0.00329 & 0.00017 & 1.10247 \\
\hline 5.55453 & 0.00415 & 0.00021 & 1.11091 \\
\hline 5.59641 & 0.00703 & 0.00036 & 1.11928 \\
\hline 5.63797 & 0.00568 & 0.00029 & 1.12759 \\
\hline 5.68016 & 0.00689 & 0.00035 & 1.13603 \\
\hline 5.72266 & 0.00892 & 0.00045 & 1.14453 \\
\hline 5.765 & 0.0062 & 0.00032 & 1.153 \\
\hline 5.80734 & 0.00553 & 0.00028 & 1.16147 \\
\hline 5.85016 & 0.00721 & 0.00037 & 1.17003 \\
\hline 5.89281 & 0.01039 & 0.00053 & 1.17856 \\
\hline 5.93531 & 0.01207 & 0.00061 & 1.18706 \\
\hline 5.97766 & 0.00726 & 0.00037 & 1.19553 \\
\hline 6.02031 & 0.0069 & 0.00035 & 1.20406 \\
\hline 6.06297 & 0.00796 & 0.00041 & 1.21259 \\
\hline 6.10531 & 0.00626 & 0.00032 & 1.22106 \\
\hline 6.14766 & 0.00549 & 0.00028 & 1.22953 \\
\hline 6.18969 & 0.00522 & 0.00027 & 1.23794 \\
\hline 6.23141 & 0.0048 & 0.00024 & 1.24628 \\
\hline 6.27328 & 0.00525 & 0.00027 & 1.25466 \\
\hline 6.31578 & 0.00601 & 0.00031 & 1.26316 \\
\hline 6.35812 & 0.00765 & 0.00039 & 1.27162 \\
\hline 6.40016 & 0.0101 & 0.00051 & 1.28003 \\
\hline 6.44234 & 0.00904 & 0.00046 & 1.28847 \\
\hline 6.48453 & 0.00576 & 0.00029 & 1.29691 \\
\hline 6.52656 & 0.0057 & 0.00029 & 1.30531 \\
\hline 6.56922 & 0.00807 & 0.00041 & 1.31384 \\
\hline 6.61219 & 0.00777 & 0.0004 & 1.32244 \\
\hline 6.655 & 0.01006 & 0.00051 & 1.331 \\
\hline 6.69766 & 0.00861 & 0.00044 & 1.33953 \\
\hline 6.73984 & 0.00604 & 0.00031 & 1.34797 \\
\hline 6.78234 & 0.00738 & 0.00038 & 1.35647 \\
\hline 6.82437 & 0.00599 & 0.0003 & 1.36487 \\
\hline 6.86594 & 0.00742 & 0.00038 & 1.37319 \\
\hline 6.90781 & 0.00939 & 0.00048 & 1.38156 \\
\hline 6.95016 & 0.00796 & 0.00041 & 1.39003 \\
\hline 6.9925 & 0.01024 & 0.00052 & 1.3985 \\
\hline 7.035 & 0.00899 & 0.00046 & 1.407 \\
\hline 7.07766 & 0.00865 & 0.00044 & 1.41553 \\
\hline 7.12047 & 0.01045 & 0.00053 & 1.42409 \\
\hline 7.16297 & 0.00687 & 0.00035 & 1.43259 \\
\hline 7.20563 & 0.00824 & 0.00042 & 1.44113 \\
\hline 7.24844 & 0.01018 & 0.00052 & 1.44969 \\
\hline 7.29094 & 0.00683 & 0.00035 & 1.45819 \\
\hline 7.33313 & 0.00826 & 0.00042 & 1.46663 \\
\hline 7.37531 & 0.00789 & 0.0004 & 1.47506 \\
\hline 7.4175 & 0.00634 & 0.00032 & 1.4835 \\
\hline 7.45906 & 0.00862 & 0.00044 & 1.49181 \\
\hline 7.50078 & 0.00505 & 0.00026 & 1.50016 \\
\hline 7.54312 & 0.00695 & 0.00035 & 1.50862 \\
\hline 7.58578 & 0.01152 & 0.00059 & 1.51716 \\
\hline 7.62828 & 0.00671 & 0.00034 & 1.52566 \\
\hline 7.67062 & 0.00783 & 0.0004 & 1.53412 \\
\hline 7.71281 & 0.00704 & 0.00036 & 1.54256 \\
\hline 7.75469 & 0.00141 & 0.00007 & 1.55094 \\
\hline 7.79672 & 0.00697 & 0.00035 & 1.55934 \\
\hline 7.83953 & 0.00873 & 0.00044 & 1.56791 \\
\hline 7.8825 & 0.00807 & 0.00041 & 1.5765 \\
\hline 7.92516 & 0.0105 & 0.00054 & 1.58503 \\
\hline 7.9675 & 0.00554 & 0.00028 & 1.5935 \\
\hline 8.01016 & 0.00857 & 0.00044 & 1.60203 \\
\hline 8.05234 & 0.01081 & 0.00055 & 1.61047 \\
\hline 8.09375 & 0.00489 & 0.00025 & 1.61875 \\
\hline 8.135 & 0.00769 & 0.00039 & 1.627 \\
\hline 8.17719 & 0.00717 & 0.00036 & 1.63544 \\
\hline 8.21984 & 0.00548 & 0.00028 & 1.64397 \\
\hline
\end{tabular}

\begin{tabular}{|c|c|c|c|}
\hline $\begin{array}{l}\text { Extension } \\
\text { [mm] }\end{array}$ & $\begin{array}{l}\text { Load } \\
\text { [N] }\end{array}$ & $\begin{array}{c}\text { Compressive stress } \\
\text { (MPa] }\end{array}$ & $\begin{array}{c}\text { Compressive strain } \\
{[\mathrm{mm} / \mathrm{mm}]}\end{array}$ \\
\hline 8.26234 & 0.00919 & 0.00047 & 1.65247 \\
\hline 8.30484 & 0.00695 & 0.00035 & 1.66097 \\
\hline 8.34734 & 0.00849 & 0.00043 & 1.66947 \\
\hline 8.38984 & 0.00968 & 0.00049 & 1.67797 \\
\hline 8.43219 & 0.00479 & 0.00024 & 1.68644 \\
\hline 8.47453 & 0.0069 & 0.00035 & 1.69491 \\
\hline 8.51703 & 0.01009 & 0.00051 & 1.70341 \\
\hline 8.55969 & 0.00811 & 0.00041 & 1.71194 \\
\hline 8.60203 & 0.0115 & 0.00059 & 1.72041 \\
\hline 8.64453 & 0.01047 & 0.00053 & 1.72891 \\
\hline 8.68656 & 0.00719 & 0.00037 & 1.73731 \\
\hline 8.72828 & 0.00676 & 0.00034 & 1.74566 \\
\hline 8.77 & 0.0071 & 0.00036 & 1.754 \\
\hline 8.81219 & 0.01067 & 0.00054 & 1.76244 \\
\hline 8.85453 & 0.01059 & 0.00054 & 1.77091 \\
\hline 8.89656 & 0.00642 & 0.00033 & 1.77931 \\
\hline 8.93891 & 0.00678 & 0.00035 & 1.78778 \\
\hline 8.98219 & 0.00694 & 0.00035 & 1.79644 \\
\hline 9.02516 & 0.00682 & 0.00035 & 1.80503 \\
\hline 9.06766 & 0.00879 & 0.00045 & 1.81353 \\
\hline 9.11016 & 0.00899 & 0.00046 & 1.82203 \\
\hline 9.15281 & 0.00701 & 0.00036 & 1.83056 \\
\hline 9.19531 & 0.00659 & 0.00034 & 1.83906 \\
\hline 9.23781 & 0.00649 & 0.00033 & 1.84756 \\
\hline 9.28 & 0.00665 & 0.00034 & 1.856 \\
\hline 9.32172 & 0.01042 & 0.00053 & 1.86434 \\
\hline 9.36312 & 0.0594 & 0.00303 & 1.87262 \\
\hline 9.40484 & 0.20958 & 0.01067 & 1.88097 \\
\hline 9.44734 & 0.49591 & 0.02526 & 1.88947 \\
\hline 9.48984 & 0.96899 & 0.04935 & 1.89797 \\
\hline 9.53219 & 1.63577 & 0.08331 & 1.90644 \\
\hline 9.57484 & 2.47278 & 0.12594 & 1.91497 \\
\hline 9.61781 & 3.4518 & 0.1758 & 1.92356 \\
\hline 9.66031 & 4.52527 & 0.23047 & 1.93206 \\
\hline 9.7025 & 5.62857 & 0.28666 & 1.9405 \\
\hline 9.74531 & 6.70415 & 0.34144 & 1.94906 \\
\hline 9.78813 & 7.60117 & 0.38712 & 1.95763 \\
\hline 9.83062 & 8.19285 & 0.41726 & 1.96612 \\
\hline 9.87266 & 8.57228 & 0.43658 & 1.97453 \\
\hline 9.91484 & 8.84507 & 0.45048 & 1.98297 \\
\hline 9.95656 & 9.18327 & 0.4677 & 1.99131 \\
\hline 9.99797 & 9.80545 & 0.49939 & 1.99959 \\
\hline 10.04 & 10.67616 & 0.54373 & 2.008 \\
\hline 10.0825 & 11.7107 & 0.59642 & 2.0165 \\
\hline 10.125 & 12.84946 & 0.65442 & 2.025 \\
\hline 10.16719 & 14.03755 & 0.71493 & 2.03344 \\
\hline 10.20953 & 15.24817 & 0.77658 & 2.04191 \\
\hline 10.25203 & 16.49222 & 0.83994 & 2.05041 \\
\hline 10.29469 & 17.7652 & 0.90477 & 2.05894 \\
\hline 10.33734 & 19.07375 & 0.97142 & 2.06747 \\
\hline 10.38 & 20.43412 & 1.0407 & 2.076 \\
\hline 10.42313 & 21.84387 & 1.1125 & 2.08463 \\
\hline 10.46594 & 23.3137 & 1.18736 & 2.09319 \\
\hline 10.50813 & 24.85001 & 1.2656 & 2.10162 \\
\hline 10.54984 & 26.44952 & 1.34706 & 2.10997 \\
\hline 10.59141 & 28.11227 & 1.43175 & 2.11828 \\
\hline 10.63297 & 29.84202 & 1.51984 & 2.12659 \\
\hline 10.67516 & 31.65908 & 1.61238 & 2.13503 \\
\hline 10.7175 & 33.58932 & 1.71069 & 2.1435 \\
\hline 10.75953 & 35.62963 & 1.8146 & 2.15191 \\
\hline 10.80188 & 37.77345 & 1.92379 & 2.16038 \\
\hline 10.84484 & 40.02516 & 2.03846 & 2.16897 \\
\hline 10.88766 & 42.40305 & 2.15957 & 2.17753 \\
\hline 10.93 & 44.90882 & 2.28719 & 2.186 \\
\hline 10.97219 & 47.52856 & 2.42061 & 2.19444 \\
\hline
\end{tabular}




\begin{tabular}{|c|c|c|c|}
\hline $\begin{array}{c}\text { Extension } \\
\text { (mm) }\end{array}$ & $\begin{array}{l}\text { Load } \\
\text { (N) }\end{array}$ & $\begin{array}{c}\text { Compressive stress } \\
\text { (MPa) }\end{array}$ & $\begin{array}{c}\text { Compressive strain } \\
\text { (mmimm) }\end{array}$ \\
\hline 11.01469 & 50.2826 & 2.56087 & 2.20294 \\
\hline 11.05688 & 53.18743 & 2.70881 & 2.21137 \\
\hline 11.09891 & 56.20848 & 2.86267 & 2.21978 \\
\hline 11.14094 & 59.34925 & 3.02263 & 2.22819 \\
\hline 11.18344 & 62.62106 & 3.18926 & 2.23669 \\
\hline 11.22531 & 66.03916 & 3.36335 & 2.24506 \\
\hline 11.26703 & 69.58332 & 3.54385 & 2.25341 \\
\hline 11.30937 & 73.24028 & 3.7301 & 2.26187 \\
\hline 11.35188 & 77.06839 & 3.92506 & 2.27038 \\
\hline 11.39406 & 81.09778 & 4.13028 & 2.27881 \\
\hline 11.43656 & 85.32386 & 4.34551 & 2.28731 \\
\hline 11.47938 & 89.75729 & 4.5713 & 2.29588 \\
\hline 11.52188 & 94.39699 & 4.8076 & 2.30438 \\
\hline 11.56437 & 99.21317 & 5.05289 & 2.31288 \\
\hline 11.60703 & 104.22002 & 5.30788 & 2.32141 \\
\hline 11.64984 & 109.46078 & 5.57479 & 2.32997 \\
\hline 11.69203 & 114.92144 & 5.8529 & 2.33841 \\
\hline 11.73422 & 120.56026 & 6.14008 & 2.34684 \\
\hline 11.77625 & 126.41331 & 6.43818 & 2.35525 \\
\hline 11.81781 & 132.50987 & 6.74867 & 2.36356 \\
\hline 11.85906 & 138.82936 & 7.07052 & 2.37181 \\
\hline 11.90109 & 145.35954 & 7.4031 & 2.38022 \\
\hline 11.94359 & 152.24341 & 7.75369 & 2.38872 \\
\hline 11.98609 & 159.57309 & 8.12699 & 2.39722 \\
\hline 12.02813 & 167.27727 & 8.51936 & 2.40563 \\
\hline 12.07063 & 175.29984 & 8.92795 & 2.41413 \\
\hline 12.11359 & 183.68768 & 9.35514 & 2.42272 \\
\hline 12.15641 & 192.56262 & 9.80713 & 2.43128 \\
\hline 12.19875 & 201.92357 & 10.28388 & 2.43975 \\
\hline 12.24125 & 211.6944 & 10.78151 & 2.44825 \\
\hline 12.28375 & 221.93576 & 11.30309 & 2.45675 \\
\hline 12.32609 & 232.68947 & 11.85078 & 2.46522 \\
\hline 12.36812 & 243.93285 & 12.4234 & 2.47362 \\
\hline 12.41016 & 255.69075 & 13.02222 & 2.48203 \\
\hline 12.45188 & 268.10425 & 13.65444 & 2.49038 \\
\hline 12.49312 & 281.2803 & 14.32549 & 2.49863 \\
\hline 12.53469 & 295.16858 & 15.03281 & 2.50694 \\
\hline 12.57672 & 309.85968 & 15.78102 & 2.51534 \\
\hline 12.61906 & 325.58652 & 16.58199 & 2.52381 \\
\hline 12.66125 & 342.37781 & 17.43716 & 2.53225 \\
\hline 12.70359 & 360.17148 & 18.34338 & 2.54072 \\
\hline 12.74594 & 378.93793 & 19.29915 & 2.54919 \\
\hline 12.78813 & 398.80508 & 20.31098 & 2.55763 \\
\hline 12.83031 & 419.84619 & 21.38259 & 2.56606 \\
\hline 12.8725 & 442.08167 & 22.51503 & 2.5745 \\
\hline 12.88937 & 451.35965 & 22.98756 & 2.57787 \\
\hline
\end{tabular}


Table D.3 Osteoblast-Containing Scaffolds Specimen 3 Raw Compression Data

\begin{tabular}{|c|c|c|c|}
\hline $\begin{array}{c}\text { Extension } \\
\text { [mm] }\end{array}$ & $\begin{array}{l}\text { Load } \\
\text { (N) }\end{array}$ & $\begin{array}{c}\text { Compressive stress } \\
\text { [MPa] }\end{array}$ & $\begin{array}{c}\text { Compressive strain } \\
\text { [mm/mm] }\end{array}$ \\
\hline 0 & 0.0015 & 0.00008 & 0 \\
\hline 0.00281 & 0.0018 & 0.00009 & 0.00056 \\
\hline 0.02344 & 0.01837 & 0.00094 & 0.00469 \\
\hline 0.06531 & 0.05728 & 0.00292 & 0.01306 \\
\hline 0.11156 & 0.08018 & 0.00408 & 0.02231 \\
\hline 0.15313 & 0.06363 & 0.00324 & 0.03063 \\
\hline 0.19578 & 0.03655 & 0.00186 & 0.03916 \\
\hline 0.24438 & 0.02616 & 0.00133 & 0.04888 \\
\hline 0.29516 & 0.0238 & 0.00121 & 0.05903 \\
\hline 0.34375 & 0.016 & 0.00081 & 0.06875 \\
\hline 0.39078 & 0.00475 & 0.00024 & 0.07816 \\
\hline 0.43797 & -0.00171 & -0.00009 & 0.08759 \\
\hline 0.48547 & -0.00075 & -0.00004 & 0.09709 \\
\hline 0.53219 & 0.00005 & 0 & 0.10644 \\
\hline 0.57719 & -0.00083 & -0.00004 & 0.11544 \\
\hline 0.62156 & -0.00253 & -0.00013 & 0.12431 \\
\hline 0.66641 & -0.00261 & -0.00013 & 0.13328 \\
\hline 0.71156 & 0.00077 & 0.00004 & 0.14231 \\
\hline 0.75625 & 0.00376 & 0.00019 & 0.15125 \\
\hline 0.8 & 0.00421 & 0.00021 & 0.16 \\
\hline 0.84375 & 0.00479 & 0.00024 & 0.16875 \\
\hline 0.8875 & 0.00601 & 0.00031 & 0.1775 \\
\hline 0.93078 & 0.0032 & 0.00016 & 0.18616 \\
\hline 0.97422 & 0.00062 & 0.00003 & 0.19484 \\
\hline 1.01828 & 0.00267 & 0.00014 & 0.20366 \\
\hline 1.06203 & 0.00439 & 0.00022 & 0.21241 \\
\hline 1.10516 & 0.00444 & 0.00023 & 0.22103 \\
\hline 1.14781 & 0.00213 & 0.00011 & 0.22956 \\
\hline 1.19031 & 0.00109 & 0.00006 & 0.23806 \\
\hline 1.23266 & 0.00152 & 0.00008 & 0.24653 \\
\hline 1.27484 & 0.00119 & 0.00006 & 0.25497 \\
\hline 1.31734 & 0.00095 & 0.00005 & 0.26347 \\
\hline 1.36 & 0.00186 & 0.00009 & 0.272 \\
\hline 1.40234 & 0.00426 & 0.00022 & 0.28047 \\
\hline 1.44516 & 0.00469 & 0.00024 & 0.28903 \\
\hline 1.48828 & 0.00421 & 0.00021 & 0.29766 \\
\hline 1.53109 & 0.00291 & 0.00015 & 0.30622 \\
\hline 1.57375 & 0.00265 & 0.00013 & 0.31475 \\
\hline 1.61641 & 0.00171 & 0.00009 & 0.32328 \\
\hline 1.65953 & 0.00097 & 0.00005 & 0.33191 \\
\hline 1.70219 & 0.00155 & 0.00008 & 0.34044 \\
\hline 1.74437 & 0.00284 & 0.00014 & 0.34887 \\
\hline 1.78672 & 0.00432 & 0.00022 & 0.35734 \\
\hline 1.82859 & 0.00455 & 0.00023 & 0.36572 \\
\hline 1.87031 & 0.00477 & 0.00024 & 0.37406 \\
\hline 1.91219 & 0.00404 & 0.00021 & 0.38244 \\
\hline 1.95469 & 0.00296 & 0.00015 & 0.39094 \\
\hline 1.99719 & 0.00314 & 0.00016 & 0.39944 \\
\hline 2.03969 & 0.00508 & 0.00026 & 0.40794 \\
\hline 2.08234 & 0.00583 & 0.0003 & 0.41647 \\
\hline 2.125 & 0.00566 & 0.00029 & 0.425 \\
\hline 2.16766 & 0.00475 & 0.00024 & 0.43353 \\
\hline 2.21016 & 0.00301 & 0.00015 & 0.44203 \\
\hline 2.25297 & 0.00402 & 0.0002 & 0.45059 \\
\hline 2.29563 & 0.00422 & 0.00021 & 0.45913 \\
\hline 2.33781 & 0.00256 & 0.00013 & 0.46756 \\
\hline 2.38016 & 0.00223 & 0.00011 & 0.47603 \\
\hline 2.42266 & 0.00288 & 0.00015 & 0.48453 \\
\hline 2.46438 & 0.00166 & 0.00008 & 0.49288 \\
\hline 2.50563 & 0.00128 & 0.00007 & 0.50113 \\
\hline 2.54766 & 0.00254 & 0.00013 & 0.50953 \\
\hline 2.59016 & 0.00467 & 0.00024 & 0.51803 \\
\hline 2.63266 & 0.00422 & 0.00021 & 0.52653 \\
\hline 2.67484 & 0.00353 & 0.00018 & 0.53497 \\
\hline 2.7175 & 0.00417 & 0.00021 & 0.5435 \\
\hline
\end{tabular}

\begin{tabular}{|c|c|c|c|}
\hline $\begin{array}{c}\text { Extension } \\
\text { (mm) }\end{array}$ & $\begin{array}{l}\text { Load } \\
\text { (N) }\end{array}$ & $\begin{array}{c}\text { Compressive stress } \\
\text { (MPa] }\end{array}$ & $\begin{array}{c}\text { Compressive strain } \\
\text { [mmimm] }\end{array}$ \\
\hline 2.80281 & 0.00344 & 0.00018 & 0.56056 \\
\hline 2.84516 & 0.00222 & 0.00011 & 0.56903 \\
\hline 2.8875 & 0.00034 & 0.00002 & 0.5775 \\
\hline 2.93 & -0.00157 & -0.00008 & 0.586 \\
\hline 2.97234 & 0.00003 & 0 & 0.59447 \\
\hline 3.01453 & 0.00505 & 0.00026 & 0.60291 \\
\hline 3.05656 & 0.00715 & 0.00036 & 0.61131 \\
\hline 3.09781 & 0.00417 & 0.00021 & 0.61956 \\
\hline 3.13937 & 0.0021 & 0.00011 & 0.62787 \\
\hline 3.18172 & 0.00446 & 0.00023 & 0.63634 \\
\hline 3.22453 & 0.00619 & 0.00032 & 0.64491 \\
\hline 3.26719 & 0.00554 & 0.00028 & 0.65344 \\
\hline 3.30969 & 0.00424 & 0.00022 & 0.66194 \\
\hline 3.35219 & 0.00199 & 0.0001 & 0.67044 \\
\hline 3.39438 & -0.00049 & -0.00003 & 0.67888 \\
\hline 3.43656 & -0.00001 & 0 & 0.68731 \\
\hline 3.47922 & 0.00224 & 0.00011 & 0.69584 \\
\hline 3.52187 & 0.00396 & 0.0002 & 0.70437 \\
\hline 3.56453 & 0.00317 & 0.00016 & 0.71291 \\
\hline 3.60703 & 0.00213 & 0.00011 & 0.72141 \\
\hline 3.64953 & 0.0021 & 0.00011 & 0.72991 \\
\hline 3.69172 & 0.00484 & 0.00025 & 0.73834 \\
\hline 3.73328 & 0.00592 & 0.0003 & 0.74666 \\
\hline 3.77516 & 0.00539 & 0.00027 & 0.75503 \\
\hline 3.81734 & 0.006 & 0.00031 & 0.76347 \\
\hline 3.85953 & 0.00624 & 0.00032 & 0.77191 \\
\hline 3.90141 & 0.00465 & 0.00024 & 0.78028 \\
\hline 3.94375 & 0.00349 & 0.00018 & 0.78875 \\
\hline 3.98688 & 0.00607 & 0.00031 & 0.79738 \\
\hline 4.02969 & 0.0075 & 0.00038 & 0.80594 \\
\hline 4.07203 & 0.00468 & 0.00024 & 0.81441 \\
\hline 4.11469 & 0.00279 & 0.00014 & 0.82294 \\
\hline 4.1575 & 0.0046 & 0.00023 & 0.8315 \\
\hline 4.20016 & 0.00587 & 0.0003 & 0.84003 \\
\hline 4.2425 & 0.00442 & 0.00023 & 0.8485 \\
\hline 4.28453 & 0.00505 & 0.00026 & 0.85691 \\
\hline 4.32625 & 0.00549 & 0.00028 & 0.86525 \\
\hline 4.36766 & 0.00456 & 0.00023 & 0.87353 \\
\hline 4.40984 & 0.00392 & 0.0002 & 0.88197 \\
\hline 4.4525 & 0.0076 & 0.00039 & 0.8905 \\
\hline 4.49484 & 0.01064 & 0.00054 & 0.89897 \\
\hline 4.53703 & 0.00813 & 0.00041 & 0.90741 \\
\hline 4.57953 & 0.00467 & 0.00024 & 0.91591 \\
\hline 4.62219 & 0.00329 & 0.00017 & 0.92444 \\
\hline 4.66469 & 0.00363 & 0.00018 & 0.93294 \\
\hline 4.7075 & 0.00474 & 0.00024 & 0.9415 \\
\hline 4.75078 & 0.00724 & 0.00037 & 0.95016 \\
\hline 4.79375 & 0.00747 & 0.00038 & 0.95875 \\
\hline 4.83594 & 0.00448 & 0.00023 & 0.96719 \\
\hline 4.87797 & 0.0023 & 0.00012 & 0.97559 \\
\hline 4.92016 & 0.00227 & 0.00012 & 0.98403 \\
\hline 4.96188 & 0.0043 & 0.00022 & 0.99238 \\
\hline 5.00328 & 0.00529 & 0.00027 & 1.00066 \\
\hline 5.045 & 0.00326 & 0.00017 & 1.009 \\
\hline 5.0875 & 0.00402 & 0.0002 & 1.0175 \\
\hline 5.13016 & 0.00705 & 0.00036 & 1.02603 \\
\hline 5.17266 & 0.00799 & 0.00041 & 1.03453 \\
\hline 5.215 & 0.00543 & 0.00028 & 1.043 \\
\hline 5.25766 & 0.00402 & 0.0002 & 1.05153 \\
\hline 5.30016 & 0.00472 & 0.00024 & 1.06003 \\
\hline 5.34234 & 0.00255 & 0.00013 & 1.06847 \\
\hline 5.385 & 0.00115 & 0.00006 & 1.077 \\
\hline 5.42813 & 0.00427 & 0.00022 & 1.08563 \\
\hline 5.47109 & 0.00635 & 0.00032 & 1.09422 \\
\hline 5.51344 & 0.0051 & 0.00026 & 1.10269 \\
\hline
\end{tabular}




\begin{tabular}{|c|c|c|c|}
\hline $\begin{array}{l}\text { Extension } \\
\text { (mm) }\end{array}$ & $\begin{array}{l}\text { Load } \\
\text { (N) }\end{array}$ & $\begin{array}{c}\text { Compressive stress } \\
\text { (MPa) }\end{array}$ & $\begin{array}{c}\text { Compressive strain } \\
{[\mathrm{mm} / \mathrm{mm})}\end{array}$ \\
\hline 5.55516 & 0.00139 & 0.00007 & 1.11103 \\
\hline 5.59672 & 0.00083 & 0.00004 & 1.11934 \\
\hline 5.63812 & 0.00294 & 0.00015 & 1.12762 \\
\hline 5.68031 & 0.00594 & 0.0003 & 1.13606 \\
\hline 5.72266 & 0.00809 & 0.00041 & 1.14453 \\
\hline 5.76484 & 0.00753 & 0.00038 & 1.15297 \\
\hline 5.80719 & 0.00576 & 0.00029 & 1.16144 \\
\hline 5.84984 & 0.0044 & 0.00022 & 1.16997 \\
\hline 5.8925 & 0.00497 & 0.00025 & 1.1785 \\
\hline 5.935 & 0.00594 & 0.0003 & 1.187 \\
\hline 5.9775 & 0.00498 & 0.00025 & 1.1955 \\
\hline 6.02031 & 0.00361 & 0.00018 & 1.20406 \\
\hline 6.06281 & 0.00222 & 0.00011 & 1.21256 \\
\hline 6.10531 & 0.00263 & 0.00013 & 1.22106 \\
\hline 6.1475 & 0.00331 & 0.00017 & 1.2295 \\
\hline 6.18953 & 0.00481 & 0.00024 & 1.23791 \\
\hline 6.23125 & 0.0057 & 0.00029 & 1.24625 \\
\hline 6.27328 & 0.00633 & 0.00032 & 1.25466 \\
\hline 6.31578 & 0.00673 & 0.00034 & 1.26316 \\
\hline 6.35813 & 0.00623 & 0.00032 & 1.27163 \\
\hline 6.40031 & 0.00663 & 0.00034 & 1.28006 \\
\hline 6.44281 & 0.00881 & 0.00045 & 1.28856 \\
\hline 6.485 & 0.00733 & 0.00037 & 1.297 \\
\hline 6.52703 & 0.00289 & 0.00015 & 1.30541 \\
\hline 6.56938 & 0.00112 & 0.00006 & 1.31388 \\
\hline 6.61219 & 0.00172 & 0.00009 & 1.32244 \\
\hline 6.65484 & 0.00349 & 0.00018 & 1.33097 \\
\hline 6.69766 & 0.00384 & 0.0002 & 1.33953 \\
\hline 6.74016 & 0.00371 & 0.00019 & 1.34803 \\
\hline 6.78266 & 0.004 & 0.0002 & 1.35653 \\
\hline 6.82422 & 0.00449 & 0.00023 & 1.36484 \\
\hline 6.86578 & 0.00594 & 0.0003 & 1.37316 \\
\hline 6.90781 & 0.00887 & 0.00045 & 1.38156 \\
\hline 6.95016 & 0.00932 & 0.00047 & 1.39003 \\
\hline 6.99234 & 0.0068 & 0.00035 & 1.39847 \\
\hline 7.03469 & 0.0051 & 0.00026 & 1.40694 \\
\hline 7.0775 & 0.00514 & 0.00026 & 1.4155 \\
\hline 7.12031 & 0.00637 & 0.00032 & 1.42406 \\
\hline 7.16281 & 0.00591 & 0.0003 & 1.43256 \\
\hline 7.20531 & 0.00567 & 0.00029 & 1.44106 \\
\hline 7.24813 & 0.00541 & 0.00028 & 1.44963 \\
\hline 7.29078 & 0.00449 & 0.00023 & 1.45816 \\
\hline 7.33297 & 0.00326 & 0.00017 & 1.46659 \\
\hline 7.37531 & 0.0038 & 0.00019 & 1.47506 \\
\hline 7.41766 & 0.00647 & 0.00033 & 1.48353 \\
\hline 7.45937 & 0.00721 & 0.00037 & 1.49187 \\
\hline 7.50125 & 0.0059 & 0.0003 & 1.50025 \\
\hline 7.54359 & 0.00662 & 0.00034 & 1.50872 \\
\hline 7.58609 & 0.00887 & 0.00045 & 1.51722 \\
\hline 7.62844 & 0.00864 & 0.00044 & 1.52569 \\
\hline 7.67047 & 0.00651 & 0.00033 & 1.53409 \\
\hline 7.7125 & 0.00627 & 0.00032 & 1.5425 \\
\hline 7.75469 & 0.00553 & 0.00028 & 1.55094 \\
\hline 7.79688 & 0.00284 & 0.00014 & 1.55938 \\
\hline 7.83938 & 0.00228 & 0.00012 & 1.56788 \\
\hline 7.8825 & 0.00433 & 0.00022 & 1.5765 \\
\hline 7.92531 & 0.00437 & 0.00022 & 1.58506 \\
\hline 7.96766 & 0.00162 & 0.00008 & 1.59353 \\
\hline 8.01 & 0.00178 & 0.00009 & 1.602 \\
\hline 8.05219 & 0.00459 & 0.00023 & 1.61044 \\
\hline 8.09375 & 0.00653 & 0.00033 & 1.61875 \\
\hline 8.135 & 0.00688 & 0.00035 & 1.627 \\
\hline 8.17734 & 0.0081 & 0.00041 & 1.63547 \\
\hline 8.21984 & 0.00873 & 0.00044 & 1.64397 \\
\hline 8.26203 & 0.00758 & 0.00039 & 1.65241 \\
\hline
\end{tabular}

\begin{tabular}{|c|c|c|c|}
\hline $\begin{array}{l}\text { Extension } \\
\text { (mm) }\end{array}$ & $\begin{array}{l}\text { Load } \\
\text { (N) }\end{array}$ & $\begin{array}{c}\text { Compressive stress } \\
\text { (MPa] }\end{array}$ & $\begin{array}{c}\text { Compressive strain } \\
\text { [mm/mm }\end{array}$ \\
\hline 8.30453 & 0.00538 & 0.00027 & 1.66091 \\
\hline 8.34734 & 0.0068 & 0.00035 & 1.66947 \\
\hline 8.39 & 0.00756 & 0.00039 & 1.678 \\
\hline 8.43219 & 0.00613 & 0.00031 & 1.68644 \\
\hline 8.47469 & 0.0038 & 0.00019 & 1.69494 \\
\hline 8.51734 & 0.00433 & 0.00022 & 1.70347 \\
\hline 8.55984 & 0.00565 & 0.00029 & 1.71197 \\
\hline 8.60219 & 0.00669 & 0.00034 & 1.72044 \\
\hline 8.64469 & 0.00658 & 0.00034 & 1.72894 \\
\hline 8.68672 & 0.00646 & 0.00033 & 1.73734 \\
\hline 8.72828 & 0.00541 & 0.00028 & 1.74566 \\
\hline 8.76984 & 0.00461 & 0.00023 & 1.75397 \\
\hline 8.81188 & 0.00591 & 0.0003 & 1.76238 \\
\hline 8.85406 & 0.00796 & 0.00041 & 1.77081 \\
\hline 8.89656 & 0.00855 & 0.00044 & 1.77931 \\
\hline 8.93953 & 0.00606 & 0.00031 & 1.78791 \\
\hline 8.9825 & 0.00432 & 0.00022 & 1.7965 \\
\hline 9.02516 & 0.00463 & 0.00024 & 1.80503 \\
\hline 9.06734 & 0.00458 & 0.00023 & 1.81347 \\
\hline 9.11 & 0.00478 & 0.00024 & 1.822 \\
\hline 9.15281 & 0.00584 & 0.0003 & 1.83056 \\
\hline 9.19547 & 0.00546 & 0.00028 & 1.83909 \\
\hline 9.2375 & 0.00338 & 0.00017 & 1.8475 \\
\hline 9.27969 & 0.00241 & 0.00012 & 1.85594 \\
\hline 9.32172 & 0.00501 & 0.00026 & 1.86434 \\
\hline 9.36328 & 0.00704 & 0.00036 & 1.87266 \\
\hline 9.40516 & 0.00709 & 0.00036 & 1.88103 \\
\hline 9.4475 & 0.00705 & 0.00036 & 1.8895 \\
\hline 9.49 & 0.00718 & 0.00037 & 1.898 \\
\hline 9.5325 & 0.00864 & 0.00044 & 1.9065 \\
\hline 9.57516 & 0.00808 & 0.00041 & 1.91503 \\
\hline 9.61781 & 0.00663 & 0.00034 & 1.92356 \\
\hline 9.66031 & 0.00526 & 0.00027 & 1.93206 \\
\hline 9.70266 & 0.00461 & 0.00023 & 1.94053 \\
\hline 9.74547 & 0.00542 & 0.00028 & 1.94909 \\
\hline 9.78813 & 0.00587 & 0.0003 & 1.95763 \\
\hline 9.83047 & 0.00775 & 0.00039 & 1.96609 \\
\hline 9.87266 & 0.00845 & 0.00043 & 1.97453 \\
\hline 9.91469 & 0.00626 & 0.00032 & 1.98294 \\
\hline 9.95656 & 0.00542 & 0.00028 & 1.99131 \\
\hline 9.99813 & 0.00693 & 0.00035 & 1.99962 \\
\hline 10.04031 & 0.00865 & 0.00044 & 2.00806 \\
\hline 10.08266 & 0.00908 & 0.00046 & 2.01653 \\
\hline 10.125 & 0.00965 & 0.00049 & 2.025 \\
\hline 10.1675 & 0.01072 & 0.00055 & 2.0335 \\
\hline 10.21031 & 0.00867 & 0.00044 & 2.04206 \\
\hline 10.25297 & 0.00938 & 0.00048 & 2.05059 \\
\hline 10.29547 & 0.02317 & 0.00118 & 2.05909 \\
\hline 10.33813 & 0.09458 & 0.00482 & 2.06763 \\
\hline 10.38094 & 0.30263 & 0.01541 & 2.07619 \\
\hline 10.42359 & 0.67239 & 0.03424 & 2.08472 \\
\hline 10.46594 & 1.1809 & 0.06014 & 2.09319 \\
\hline 10.50813 & 1.79306 & 0.09132 & 2.10162 \\
\hline 10.55 & 2.47592 & 0.1261 & 2.11 \\
\hline 10.59141 & 3.20054 & 0.163 & 2.11828 \\
\hline 10.63281 & 3.94944 & 0.20114 & 2.12656 \\
\hline 10.675 & 4.71454 & 0.24011 & 2.135 \\
\hline 10.71734 & 5.50082 & 0.28015 & 2.14347 \\
\hline 10.75953 & 6.31992 & 0.32187 & 2.15191 \\
\hline 10.80203 & 7.13323 & 0.36329 & 2.16041 \\
\hline 10.84484 & 7.77324 & 0.39589 & 2.16897 \\
\hline 10.88766 & 8.18921 & 0.41707 & 2.17753 \\
\hline 10.93016 & 8.47413 & 0.43158 & 2.18603 \\
\hline 10.97281 & 8.72018 & 0.44411 & 2.19456 \\
\hline 11.01562 & 9.17116 & 0.46708 & 2.20312 \\
\hline
\end{tabular}




\begin{tabular}{|c|c|c|c|}
\hline $\begin{array}{l}\text { Extension } \\
\text { (mm) }\end{array}$ & $\begin{array}{l}\text { Load } \\
\text { (N) }\end{array}$ & $\begin{array}{c}\text { Compressive stress } \\
\text { (MPa) }\end{array}$ & $\begin{array}{c}\text { Compressive strain } \\
{[\mathrm{mm} / \mathrm{mm}]}\end{array}$ \\
\hline 11.09984 & 10.86935 & 0.55357 & 2.21997 \\
\hline 11.14187 & 11.97989 & 0.61013 & 2.22837 \\
\hline 11.18406 & 13.19842 & 0.67219 & 2.23681 \\
\hline 11.22578 & 14.50222 & 0.73859 & 2.24516 \\
\hline 11.26766 & 15.88015 & 0.80877 & 2.25353 \\
\hline 11.31 & 17.33459 & 0.88284 & 2.262 \\
\hline 11.3525 & 18.88697 & 0.96191 & 2.2705 \\
\hline 11.39484 & 20.54773 & 1.04649 & 2.27897 \\
\hline 11.43734 & 22.32336 & 1.13692 & 2.28747 \\
\hline 11.48 & 24.22046 & 1.23354 & 2.296 \\
\hline 11.52266 & 26.25404 & 1.33711 & 2.30453 \\
\hline 11.565 & 28.42626 & 1.44774 & 2.313 \\
\hline 11.6075 & 30.72808 & 1.56497 & 2.3215 \\
\hline 11.65016 & 33.15852 & 1.68875 & 2.33003 \\
\hline 11.6925 & 35.71856 & 1.81913 & 2.3385 \\
\hline 11.73469 & 38.41426 & 1.95642 & 2.34694 \\
\hline 11.77703 & 41.27765 & 2.10225 & 2.35541 \\
\hline 11.81906 & 44.3418 & 2.25831 & 2.36381 \\
\hline 11.86047 & 47.6352 & 2.42604 & 2.37209 \\
\hline 11.90203 & 51.16899 & 2.60601 & 2.38041 \\
\hline 11.94453 & 54.99373 & 2.80081 & 2.38891 \\
\hline 11.98687 & 59.17081 & 3.01354 & 2.39737 \\
\hline 12.02906 & 63.71133 & 3.24479 & 2.40581 \\
\hline 12.07172 & 68.62314 & 3.49495 & 2.41434 \\
\hline 12.11453 & 73.91726 & 3.76458 & 2.42291 \\
\hline 12.15688 & 79.6123 & 4.05462 & 2.43138 \\
\hline 12.19922 & 85.69425 & 4.36437 & 2.43984 \\
\hline 12.24203 & 92.15021 & 4.69317 & 2.44841 \\
\hline 12.28484 & 99.0623 & 5.0452 & 2.45697 \\
\hline 12.32703 & 106.48797 & 5.42339 & 2.46541 \\
\hline 12.36891 & 114.42158 & 5.82744 & 2.47378 \\
\hline 12.41125 & 122.87756 & 6.2581 & 2.48225 \\
\hline 12.45297 & 131.93806 & 6.71955 & 2.49059 \\
\hline 12.49422 & 141.62378 & 7.21284 & 2.49884 \\
\hline 12.53594 & 151.9353 & 7.738 & 2.50719 \\
\hline 12.57812 & 163.00847 & 8.30195 & 2.51562 \\
\hline 12.62016 & 174.9939 & 8.91237 & 2.52403 \\
\hline 12.66219 & 187.86858 & 9.56807 & 2.53244 \\
\hline 12.70437 & 201.62482 & 10.26867 & 2.54087 \\
\hline 12.74688 & 216.37114 & 11.01969 & 2.54938 \\
\hline 12.78938 & 232.28699 & 11.83028 & 2.55788 \\
\hline 12.83156 & 249.50214 & 12.70704 & 2.56631 \\
\hline 12.87406 & 268.12628 & 13.65556 & 2.57481 \\
\hline 12.91656 & 288.33636 & 14.68485 & 2.58331 \\
\hline 12.95859 & 310.21561 & 15.79915 & 2.59172 \\
\hline 13.00047 & 333.8606 & 17.00338 & 2.60009 \\
\hline 13.04219 & 359.39578 & 18.30388 & 2.60844 \\
\hline 13.08328 & 386.99051 & 19.70926 & 2.61666 \\
\hline 13.12422 & 416.90613 & 21.23285 & 2.62484 \\
\hline 13.14062 & 429.55182 & 21.87689 & 2.62812 \\
\hline
\end{tabular}


Table D.4 Osteoblast-Containing Scaffolds Specimen 4 Raw Compression Data

\begin{tabular}{|c|c|c|c|c|c|c|c|}
\hline $\begin{array}{l}\text { Extension } \\
\text { (mm) }\end{array}$ & $\begin{array}{c}\text { Load } \\
\text { (N) }\end{array}$ & $\begin{array}{c}\text { Compressive stress } \\
\text { (MPa] }\end{array}$ & $\begin{array}{c}\text { Compressive strain } \\
\text { (mmimm) }\end{array}$ & $\begin{array}{c}\begin{array}{c}\text { Extension } \\
(\mathrm{mm})\end{array} \\
\end{array}$ & $\begin{array}{c}\text { Load } \\
\text { (N) }\end{array}$ & $\begin{array}{c}\text { Compressive stress } \\
\text { (MPa] }\end{array}$ & $\begin{array}{c}\text { Compressive strain } \\
{[\mathrm{mm} / \mathrm{mm}]}\end{array}$ \\
\hline 0 & 0.00422 & 0.00021 & 0 & 2.76031 & 0.00277 & 0.00014 & 0.55206 \\
\hline 0.00281 & 0.00466 & 0.00024 & 0.00056 & 2.80297 & 0.00218 & 0.00011 & 0.56059 \\
\hline 0.02359 & 0.0188 & 0.00096 & 0.00472 & 2.84516 & 0.00236 & 0.00012 & 0.56903 \\
\hline 0.06531 & 0.05631 & 0.00287 & 0.01306 & 2.88719 & 0.00351 & 0.00018 & 0.57744 \\
\hline 0.11125 & 0.08157 & 0.00415 & 0.02225 & 2.92953 & 0.0053 & 0.00027 & 0.58591 \\
\hline 0.15297 & 0.06582 & 0.00335 & 0.03059 & 2.97219 & 0.00514 & 0.00026 & 0.59444 \\
\hline 0.19578 & 0.034 & 0.00173 & 0.03916 & 3.01469 & 0.00301 & 0.00015 & 0.60294 \\
\hline 0.24438 & 0.02389 & 0.00122 & 0.04888 & 3.05672 & 0.00344 & 0.00018 & 0.61134 \\
\hline 0.29516 & 0.02236 & 0.00114 & 0.05903 & 3.09828 & 0.00479 & 0.00024 & 0.61966 \\
\hline 0.34375 & 0.01165 & 0.00059 & 0.06875 & 3.14 & 0.00273 & 0.00014 & 0.628 \\
\hline 0.39078 & 0.00066 & 0.00003 & 0.07816 & 3.1825 & 0.00395 & 0.0002 & 0.6365 \\
\hline 0.43797 & -0.00001 & 0 & 0.08759 & 3.225 & 0.00693 & 0.00035 & 0.645 \\
\hline 0.48547 & -0.00049 & -0.00003 & 0.09709 & 3.26734 & 0.00411 & 0.00021 & 0.65347 \\
\hline 0.53203 & -0.00123 & -0.00006 & 0.10641 & 3.30969 & 0.00229 & 0.00012 & 0.66194 \\
\hline 0.57719 & -0.002 & -0.0001 & 0.11544 & 3.35219 & 0.00264 & 0.00013 & 0.67044 \\
\hline 0.62156 & -0.00176 & -0.00009 & 0.12431 & 3.39406 & 0.00348 & 0.00018 & 0.67881 \\
\hline 0.66656 & -0.00106 & -0.00005 & 0.13331 & 3.43609 & 0.00259 & 0.00013 & 0.68722 \\
\hline 0.71156 & 0.00277 & 0.00014 & 0.14231 & 3.47875 & 0.00145 & 0.00007 & 0.69575 \\
\hline 0.75594 & 0.00629 & 0.00032 & 0.15119 & 3.52187 & 0.00239 & 0.00012 & 0.70437 \\
\hline 0.79969 & 0.00164 & 0.00008 & 0.15994 & 3.56484 & 0.00357 & 0.00018 & 0.71297 \\
\hline 0.84359 & 0.0022 & 0.00011 & 0.16872 & 3.60734 & 0.00346 & 0.00018 & 0.72147 \\
\hline 0.8875 & 0.00287 & 0.00015 & 0.1775 & 3.64937 & 0.00236 & 0.00012 & 0.72987 \\
\hline 0.93094 & 0.00155 & 0.00008 & 0.18619 & 3.69125 & 0.00074 & 0.00004 & 0.73825 \\
\hline 0.97422 & 0.00006 & 0 & 0.19484 & 3.73297 & 0.00128 & 0.00007 & 0.74659 \\
\hline 1.01828 & 0.00027 & 0.00001 & 0.20366 & 3.77484 & 0.00284 & 0.00014 & 0.75497 \\
\hline 1.06219 & -0.00018 & -0.00001 & 0.21244 & 3.8175 & 0.00293 & 0.00015 & 0.7635 \\
\hline 1.10547 & -0.0003 & $\begin{array}{l}-0.00002 \\
\end{array}$ & 0.22109 & 3.86016 & 0.00516 & 0.00026 & 0.77203 \\
\hline 1.14813 & 0.00174 & 0.00009 & 0.22963 & 3.90219 & 0.00621 & 0.00032 & 0.78044 \\
\hline 1.19063 & 0.0019 & 0.0001 & 0.23813 & 3.94453 & 0.00441 & 0.00022 & 0.78891 \\
\hline 1.23234 & 0.00211 & 0.00011 & 0.24647 & 3.98703 & 0.00201 & 0.0001 & 0.79741 \\
\hline 1.27437 & -0.00026 & -0.00001 & 0.25488 & 4.02953 & 0.00221 & 0.00011 & 0.80591 \\
\hline 1.31703 & -0.00009 & 0 & 0.26341 & 4.07219 & 0.00442 & 0.00023 & 0.81444 \\
\hline 1.36 & 0.00339 & 0.00017 & 0.272 & 4.11484 & 0.00436 & 0.00022 & 0.82297 \\
\hline 1.4025 & 0.00448 & 0.00023 & 0.2805 & 4.15734 & 0.00354 & 0.00018 & 0.83147 \\
\hline 1.44516 & 0.00562 & 0.00029 & 0.28903 & 4.19969 & 0.00429 & 0.00022 & 0.83994 \\
\hline 1.48813 & 0.00668 & 0.00034 & 0.29763 & 4.24234 & 0.00496 & 0.00025 & 0.84847 \\
\hline 1.53109 & 0.00432 & 0.00022 & 0.30622 & 4.28484 & 0.00361 & 0.00018 & 0.85697 \\
\hline 1.57375 & 0.00348 & 0.00018 & 0.31475 & 4.32656 & 0.00265 & 0.00014 & 0.86531 \\
\hline 1.61656 & 0.00235 & 0.00012 & 0.32331 & 4.36781 & 0.00329 & 0.00017 & 0.87356 \\
\hline 1.65922 & 0.00293 & 0.00015 & 0.33184 & 4.40953 & 0.00347 & 0.00018 & 0.88191 \\
\hline 1.70203 & 0.00427 & 0.00022 & 0.34041 & 4.45203 & 0.00374 & 0.00019 & 0.89041 \\
\hline 1.74453 & 0.00447 & 0.00023 & 0.34891 & 4.49438 & 0.00458 & 0.00023 & 0.89888 \\
\hline 1.78688 & 0.00362 & 0.00018 & 0.35737 & 4.53688 & 0.00462 & 0.00024 & 0.90738 \\
\hline 1.82859 & 0.00276 & 0.00014 & 0.36572 & 4.58 & 0.00509 & 0.00026 & 0.916 \\
\hline 1.87047 & 0.00287 & 0.00015 & 0.37409 & 4.62297 & 0.00638 & 0.00032 & 0.92459 \\
\hline 1.9125 & 0.00356 & 0.00018 & 0.3825 & 4.66547 & 0.00592 & 0.0003 & 0.93309 \\
\hline 1.95484 & 0.00472 & 0.00024 & 0.39097 & 4.70781 & 0.00279 & 0.00014 & 0.94156 \\
\hline 1.99719 & 0.00384 & 0.00002 & 0.39944 & 4.75063 & 0.0019 & 0.0001 & 0.95013 \\
\hline 2.03938 & 0.00348 & 0.00018 & 0.40788 & 4.79344 & 0.00349 & 0.00018 & 0.95869 \\
\hline 2.08172 & 0.00297 & 0.00015 & 0.41634 & 4.83578 & 0.00496 & 0.00025 & 0.96716 \\
\hline 2.12469 & 0.00242 & 0.00012 & 0.42494 & 4.87797 & 0.00574 & 0.00029 & 0.97559 \\
\hline 2.1675 & 0.00481 & 0.00025 & 0.4335 & 4.92 & 0.00539 & 0.00027 & 0.984 \\
\hline 2.21031 & 0.00578 & 0.00029 & 0.44206 & 4.96188 & 0.00363 & 0.00018 & 0.99238 \\
\hline 2.25312 & 0.00562 & 0.00029 & 0.45063 & 5.00344 & 0.00221 & 0.00011 & 1.00069 \\
\hline 2.29578 & 0.00372 & 0.00019 & 0.45916 & 5.04547 & 0.00263 & 0.00013 & 1.00909 \\
\hline 2.33797 & 0.00218 & 0.00011 & 0.46759 & 5.08781 & 0.00218 & 0.00011 & 1.01756 \\
\hline 2.38016 & 0.00443 & 0.00023 & 0.47603 & 5.13016 & 0.00296 & 0.00015 & 1.02603 \\
\hline 2.42219 & 0.00645 & 0.00033 & 0.48444 & 5.17219 & 0.00378 & 0.00019 & 1.03444 \\
\hline 2.46391 & 0.00598 & 0.0003 & 0.49278 & 5.21453 & 0.00371 & 0.00019 & 1.04291 \\
\hline 2.50547 & 0.00336 & 0.00017 & 0.50109 & 5.25719 & 0.00366 & 0.00019 & 1.05144 \\
\hline 2.5475 & 0.00102 & 0.00005 & 0.5095 & 5.30016 & 0.00656 & 0.00033 & 1.06003 \\
\hline 2.59 & 0.0009 & 0.00005 & 0.518 & 5.34313 & 0.0081 & 0.00041 & 1.06863 \\
\hline 2.6325 & 0.00128 & 0.00007 & 0.5265 & 5.38594 & 0.00384 & 0.0002 & 1.07719 \\
\hline 2.675 & 0.00139 & 0.00007 & 0.535 & 5.42844 & -0.00012 & $\begin{array}{c}0.0002 \\
-0.00001\end{array}$ & 1.08569 \\
\hline 2.71766 & 0.00185 & 0.00009 & 0.54353 & 5.47078 & 0.00024 & 0.00001 & 1.09416 \\
\hline
\end{tabular}




\begin{tabular}{|c|c|c|c|}
\hline $\begin{array}{l}\text { Extension } \\
\text { (mm) }\end{array}$ & $\begin{array}{c}\text { Load } \\
\text { (N) }\end{array}$ & \begin{tabular}{|c|} 
Compressive stress \\
(MPa)
\end{tabular} & $\begin{array}{c}\text { Compressive strain } \\
{[\mathrm{mm} / \mathrm{mm}]}\end{array}$ \\
\hline 5.55516 & 0.00384 & 0.0002 & 1.11103 \\
\hline 5.59625 & 0.00365 & 0.00019 & 1.11925 \\
\hline 5.6375 & 0.00401 & 0.0002 & 1.1275 \\
\hline 5.67969 & 0.0048 & 0.00024 & 1.13594 \\
\hline 5.72234 & 0.00353 & 0.00018 & 1.14447 \\
\hline 5.76469 & 0.00283 & 0.00014 & 1.15294 \\
\hline 5.80719 & 0.00476 & 0.00024 & 1.16144 \\
\hline 5.84984 & 0.00476 & 0.00024 & 1.16997 \\
\hline 5.89266 & 0.00473 & 0.00024 & 1.17853 \\
\hline 5.935 & 0.0047 & 0.00024 & 1.187 \\
\hline 5.9775 & 0.00654 & 0.00033 & 1.1955 \\
\hline 6.02031 & 0.00833 & 0.00042 & 1.20406 \\
\hline 6.06297 & 0.00701 & 0.00036 & 1.21259 \\
\hline 6.10516 & 0.00502 & 0.00026 & 1.22103 \\
\hline 6.1475 & 0.00279 & 0.00014 & 1.2295 \\
\hline 6.18953 & 0.00169 & 0.00009 & 1.23791 \\
\hline 6.23141 & 0.00106 & 0.00005 & 1.24628 \\
\hline 6.27344 & 0.00238 & 0.00012 & 1.25469 \\
\hline 6.31594 & 0.0063 & 0.00032 & 1.26319 \\
\hline 6.35828 & 0.00812 & 0.00041 & 1.27166 \\
\hline 6.40031 & 0.00618 & 0.00031 & 1.28006 \\
\hline 6.44266 & 0.00399 & 0.0002 & 1.28853 \\
\hline 6.48469 & 0.00371 & 0.00019 & 1.29694 \\
\hline 6.52656 & 0.00432 & 0.00022 & 1.30531 \\
\hline 6.56906 & 0.00464 & 0.00024 & 1.31381 \\
\hline 6.61234 & 0.00637 & 0.00032 & 1.32247 \\
\hline 6.65547 & 0.00727 & 0.00037 & 1.33109 \\
\hline 6.69797 & 0.00582 & 0.0003 & 1.33959 \\
\hline 6.74031 & 0.004 & 0.0002 & 1.34806 \\
\hline 6.78266 & 0.00396 & 0.0002 & 1.35653 \\
\hline 6.82438 & 0.00406 & 0.00021 & 1.36488 \\
\hline 6.86594 & 0.00165 & 0.00008 & 1.37319 \\
\hline 6.90766 & 0.00171 & 0.00009 & 1.38153 \\
\hline 6.95 & 0.00529 & 0.00027 & 1.39 \\
\hline 6.99219 & 0.00708 & 0.00036 & 1.39844 \\
\hline 7.03453 & 0.00749 & 0.00038 & 1.40691 \\
\hline 7.0775 & 0.00787 & 0.0004 & 1.4155 \\
\hline 7.12047 & 0.00768 & 0.00039 & 1.42409 \\
\hline 7.16297 & 0.00588 & 0.0003 & 1.43259 \\
\hline 7.20547 & 0.00386 & 0.0002 & 1.44109 \\
\hline 7.24813 & 0.00403 & 0.00021 & 1.44963 \\
\hline 7.29078 & 0.00518 & 0.00026 & 1.45816 \\
\hline 7.33313 & 0.00518 & 0.00026 & 1.46662 \\
\hline 7.37547 & 0.0046 & 0.00023 & 1.47509 \\
\hline 7.41781 & 0.0048 & 0.00024 & 1.48356 \\
\hline 7.45969 & 0.00549 & 0.00028 & 1.49194 \\
\hline 7.50125 & 0.00495 & 0.00025 & 1.50025 \\
\hline 7.54344 & 0.00416 & 0.00021 & 1.50869 \\
\hline 7.58594 & 0.00592 & 0.0003 & 1.51719 \\
\hline 7.62813 & 0.00732 & 0.00037 & 1.52562 \\
\hline 7.67047 & 0.00636 & 0.00032 & 1.53409 \\
\hline 7.71266 & 0.00395 & 0.0002 & 1.54253 \\
\hline 7.75453 & 0.0048 & 0.00024 & 1.55091 \\
\hline 7.79672 & 0.0077 & 0.00039 & 1.55934 \\
\hline 7.83938 & 0.00688 & 0.00035 & 1.56788 \\
\hline 7.8825 & 0.00693 & 0.00035 & 1.5765 \\
\hline 7.92531 & 0.00757 & 0.00039 & 1.58506 \\
\hline 7.96766 & 0.00616 & 0.00031 & 1.59353 \\
\hline 8.00984 & 0.0041 & 0.00021 & 1.60197 \\
\hline 8.05188 & 0.00269 & 0.00014 & 1.61038 \\
\hline 8.09359 & 0.00378 & 0.00019 & 1.61872 \\
\hline 8.13547 & 0.00389 & 0.0002 & 1.62709 \\
\hline 8.1775 & 0.00475 & 0.00024 & 1.6355 \\
\hline 8.22 & 0.00825 & 0.00042 & 1.644 \\
\hline 8.26234 & 0.00888 & 0.00045 & 1.65247 \\
\hline
\end{tabular}

\begin{tabular}{|c|c|c|c|}
\hline $\begin{array}{l}\text { Extension } \\
\text { (mm) }\end{array}$ & $\begin{array}{l}\text { Load } \\
\text { [N] }\end{array}$ & $\begin{array}{c}\text { Compressive stress } \\
\text { (MPa] }\end{array}$ & $\begin{array}{c}\text { Compressive strain } \\
\text { [mmimm }\end{array}$ \\
\hline 8.47437 & 0.00444 & 0.00023 & 1.69488 \\
\hline 8.51688 & 0.00533 & 0.00027 & 1.70338 \\
\hline 8.55953 & 0.00549 & 0.00028 & 1.71191 \\
\hline 8.60203 & 0.00565 & 0.00029 & 1.72041 \\
\hline 8.64437 & 0.00482 & 0.00025 & 1.72887 \\
\hline 8.68656 & 0.00306 & 0.00016 & 1.73731 \\
\hline 8.72813 & 0.0028 & 0.00014 & 1.74563 \\
\hline 8.76969 & 0.00463 & 0.00024 & 1.75394 \\
\hline 8.81219 & 0.00736 & 0.00037 & 1.76244 \\
\hline 8.855 & 0.00905 & 0.00046 & 1.771 \\
\hline 8.89734 & 0.0089 & 0.00045 & 1.77947 \\
\hline 8.93969 & 0.0082 & 0.00042 & 1.78794 \\
\hline 8.9825 & 0.00691 & 0.00035 & 1.7965 \\
\hline 9.02547 & 0.00582 & 0.0003 & 1.80509 \\
\hline 9.06781 & 0.00556 & 0.00028 & 1.81356 \\
\hline 9.11 & 0.00536 & 0.00027 & 1.822 \\
\hline 9.15266 & 0.00572 & 0.00029 & 1.83053 \\
\hline 9.19516 & 0.00622 & 0.00032 & 1.83903 \\
\hline 9.23766 & 0.00729 & 0.00037 & 1.84753 \\
\hline 9.27984 & 0.00473 & 0.00024 & 1.85597 \\
\hline 9.32141 & 0.00162 & 0.00008 & 1.86428 \\
\hline 9.36297 & 0.00096 & 0.00005 & 1.87259 \\
\hline 9.40484 & 0.00216 & 0.00011 & 1.88097 \\
\hline 9.44734 & 0.00552 & 0.00028 & 1.88947 \\
\hline 9.48969 & 0.00835 & 0.00043 & 1.89794 \\
\hline 9.53203 & 0.00993 & 0.00051 & 1.90641 \\
\hline 9.57484 & 0.01037 & 0.00053 & 1.91497 \\
\hline 9.61766 & 0.0093 & 0.00047 & 1.92353 \\
\hline 9.66031 & 0.01105 & 0.00056 & 1.93206 \\
\hline 9.70266 & 0.01512 & 0.00077 & 1.94053 \\
\hline 9.74531 & 0.02056 & 0.00105 & 1.94906 \\
\hline 9.78828 & 0.02514 & 0.00128 & 1.95766 \\
\hline 9.83094 & 0.02702 & 0.00138 & 1.96619 \\
\hline 9.87313 & 0.03791 & 0.00193 & 1.97462 \\
\hline 9.91516 & 0.07016 & 0.00357 & 1.98303 \\
\hline 9.95687 & 0.12962 & 0.0066 & 1.99137 \\
\hline 9.99828 & 0.21849 & 0.01113 & 1.99966 \\
\hline 10.04047 & 0.33131 & 0.01687 & 2.00809 \\
\hline 10.08297 & 0.46436 & 0.02365 & 2.01659 \\
\hline 10.12516 & 0.64105 & 0.03265 & 2.02503 \\
\hline 10.16734 & 0.89124 & 0.04539 & 2.03347 \\
\hline 10.20984 & 1.22059 & 0.06216 & 2.04197 \\
\hline 10.25266 & 1.61758 & 0.08238 & 2.05053 \\
\hline 10.29516 & 2.06997 & 0.10542 & 2.05903 \\
\hline 10.33797 & 2.56661 & 0.13072 & 2.06759 \\
\hline 10.38094 & 3.10083 & 0.15792 & 2.07619 \\
\hline 10.42375 & 3.6738 & 0.18711 & 2.08475 \\
\hline 10.46609 & 4.28898 & 0.21844 & 2.09322 \\
\hline 10.50813 & 4.95344 & 0.25228 & 2.10162 \\
\hline 10.54984 & 5.66386 & 0.28846 & 2.10997 \\
\hline 10.59141 & 6.41893 & 0.32691 & 2.11828 \\
\hline 10.63297 & 7.22088 & 0.36776 & 2.12659 \\
\hline 10.675 & 8.06362 & 0.41068 & 2.135 \\
\hline 10.71719 & 8.92955 & 0.45478 & 2.14344 \\
\hline 10.75953 & 9.68706 & 0.49336 & 2.15191 \\
\hline 10.80219 & 10.20278 & 0.51962 & 2.16044 \\
\hline 10.84531 & 10.53911 & 0.53675 & 2.16906 \\
\hline 10.88812 & 10.80119 & 0.5501 & 2.17762 \\
\hline 10.93047 & 11.12034 & 0.56635 & 2.18609 \\
\hline 10.97266 & 11.78441 & 0.60018 & 2.19453 \\
\hline 11.01547 & 12.74038 & 0.64886 & 2.20309 \\
\hline 11.05781 & 13.89144 & 0.70749 & 2.21156 \\
\hline 11.09969 & 15.16266 & 0.77223 & 2.21994 \\
\hline 11.14172 & 16.51955 & 0.84133 & 2.22834 \\
\hline 11.18391 & 17.95789 & 0.91459 & 2.23678 \\
\hline
\end{tabular}




\begin{tabular}{|c|c|c|c|}
\hline $\begin{array}{c}\text { Extension } \\
\text { (mm) }\end{array}$ & $\begin{array}{c}\text { Load } \\
\text { (N) }\end{array}$ & $\begin{array}{c}\text { Compressive stress } \\
\text { (MPa) }\end{array}$ & $\begin{array}{c}\text { Compressive strain } \\
\text { (mmimm) }\end{array}$ \\
\hline 11.90188 & 63.43394 & 3.23066 & 2.38038 \\
\hline 11.94422 & 67.97362 & 3.46187 & 2.38884 \\
\hline 11.98656 & 72.87936 & 3.71172 & 2.39731 \\
\hline 12.02875 & 78.16408 & 3.98086 & 2.40575 \\
\hline 12.07141 & 83.87929 & 4.27194 & 2.41428 \\
\hline 12.11437 & 90.04661 & 4.58604 & 2.42287 \\
\hline 12.15672 & 96.67419 & 4.92358 & 2.43134 \\
\hline 12.19875 & 103.72972 & 5.28291 & 2.43975 \\
\hline 12.24141 & 111.17783 & 5.66224 & 2.44828 \\
\hline 12.28422 & 119.09961 & 6.06569 & 2.45684 \\
\hline 12.32656 & 127.55227 & 6.49618 & 2.46531 \\
\hline 12.36859 & 136.51015 & 6.9524 & 2.47372 \\
\hline 12.41078 & 145.98483 & 7.43495 & 2.48216 \\
\hline 12.4525 & 156.09439 & 7.94982 & 2.4905 \\
\hline 12.49391 & 166.88609 & 8.49944 & 2.49878 \\
\hline 12.53563 & 178.38965 & 9.08531 & 2.50713 \\
\hline 12.57781 & 190.76674 & 9.71567 & 2.51556 \\
\hline 12.61984 & 204.20169 & 10.39991 & 2.52397 \\
\hline 12.66187 & 218.71611 & 11.13912 & 2.53237 \\
\hline 12.70406 & 234.347 & 11.93519 & 2.54081 \\
\hline 12.74641 & 251.18568 & 12.79278 & 2.54928 \\
\hline 12.78891 & 269.39114 & 13.71998 & 2.55778 \\
\hline 12.83109 & 289.09979 & 14.72373 & 2.56622 \\
\hline 12.87328 & 310.37128 & 15.80708 & 2.57466 \\
\hline 12.91578 & 333.29208 & 16.97443 & 2.58316 \\
\hline 12.95797 & 358.00687 & 18.23314 & 2.59159 \\
\hline 12.99984 & 384.6499 & 19.59006 & 2.59997 \\
\hline 13.04156 & 413.22308 & 21.04528 & 2.60831 \\
\hline 13.08266 & 443.65094 & 22.59496 & 2.61653 \\
\hline 13.09891 & 456.3707 & 23.24277 & 2.61978 \\
\hline & & & \\
\hline & & & \\
\hline
\end{tabular}


Table D.5 ODM-Soaked Scaffolds Without Osteoblasts Specimen 1 Raw Compression Data

\begin{tabular}{|c|c|c|c|}
\hline $\begin{array}{l}\text { Extension } \\
\text { (mm) }\end{array}$ & $\begin{array}{c}\text { Load } \\
\text { (N) }\end{array}$ & \begin{tabular}{|c} 
Compressive stress \\
(MPa)
\end{tabular} & $\begin{array}{c}\text { Compressive strain } \\
{[\mathrm{mm} / \mathrm{mm})}\end{array}$ \\
\hline 0 & -0.0025 & $\begin{array}{c}-0.00013 \\
\end{array}$ & 0 \\
\hline 0.00281 & -0.00006 & 0 & 0.00056 \\
\hline 0.02344 & 0.0173 & 0.00088 & 0.00469 \\
\hline 0.06531 & 0.05381 & 0.00274 & 0.01306 \\
\hline 0.11141 & 0.07609 & 0.00388 & 0.02228 \\
\hline 0.15297 & 0.06169 & 0.00314 & 0.03059 \\
\hline 0.19594 & 0.0347 & 0.00177 & 0.03919 \\
\hline 0.24484 & 0.02307 & 0.00117 & 0.04897 \\
\hline 0.29547 & 0.02065 & 0.00105 & 0.05909 \\
\hline 0.34406 & 0.01348 & 0.00069 & 0.06881 \\
\hline 0.39109 & 0.0015 & 0.00008 & 0.07822 \\
\hline 0.43812 & -0.00483 & -0.00025 & 0.08762 \\
\hline 0.48547 & -0.00358 & $\begin{array}{c}-0.00018 \\
\end{array}$ & 0.09709 \\
\hline 0.53203 & -0.00178 & -0.00009 & 0.10641 \\
\hline 0.57719 & -0.0026 & -0.00013 & 0.11544 \\
\hline 0.62156 & -0.00473 & -0.00024 & 0.12431 \\
\hline 0.66641 & $\begin{array}{c}-0.00418 \\
\end{array}$ & -0.00021 & 0.13328 \\
\hline 0.71141 & -0.00014 & -0.00001 & 0.14228 \\
\hline 0.75594 & 0.00128 & 0.00007 & 0.15119 \\
\hline 0.79984 & 0.00004 & 0 & 0.15997 \\
\hline 0.84375 & 0.00057 & 0.00003 & 0.16875 \\
\hline 0.88734 & 0.00116 & 0.00006 & 0.17747 \\
\hline 0.93063 & -0.00153 & -0.00008 & 0.18613 \\
\hline 0.97406 & -0.00423 & -0.00022 & 0.19481 \\
\hline 1.01812 & -0.00044 & -0.00002 & 0.20362 \\
\hline 1.06203 & 0.00294 & 0.00015 & 0.21241 \\
\hline 1.10547 & 0.00079 & 0.00004 & 0.22109 \\
\hline 1.14828 & -0.0017 & -0.00009 & 0.22966 \\
\hline 1.19063 & -0.00083 & -0.00004 & 0.23813 \\
\hline 1.23266 & -0.0003 & -0.00002 & 0.24653 \\
\hline 1.27484 & -0.0014 & -0.00007 & 0.25497 \\
\hline 1.3175 & 0.00046 & 0.00002 & 0.2635 \\
\hline 1.36031 & 0.00286 & 0.00015 & 0.27206 \\
\hline 1.40281 & 0.00254 & 0.00013 & 0.28056 \\
\hline 1.44531 & 0.00046 & 0.00002 & 0.28906 \\
\hline 1.48813 & 0.00124 & 0.00006 & 0.29763 \\
\hline 1.53094 & 0.00293 & 0.00015 & 0.30619 \\
\hline 1.57359 & 0.00176 & 0.00009 & 0.31472 \\
\hline 1.61656 & -0.00042 & -0.00002 & 0.32331 \\
\hline 1.65953 & $\begin{array}{l}-0.00098 \\
\end{array}$ & -0.00005 & 0.33191 \\
\hline 1.70203 & 0.00043 & 0.00002 & 0.34041 \\
\hline 1.74437 & 0.00233 & 0.00012 & 0.34887 \\
\hline 1.78672 & 0.00236 & 0.00012 & 0.35734 \\
\hline 1.82859 & 0.00129 & 0.00007 & 0.36572 \\
\hline 1.87031 & 0.00122 & 0.00006 & 0.37406 \\
\hline 1.91234 & 0.00063 & 0.00003 & 0.38247 \\
\hline 1.95484 & 0.00063 & 0.00003 & 0.39097 \\
\hline 1.9975 & 0.00375 & 0.00019 & 0.3995 \\
\hline 2.03984 & 0.00417 & 0.00021 & 0.40797 \\
\hline 2.08234 & 0.0019 & 0.0001 & 0.41647 \\
\hline 2.12516 & 0.00023 & 0.00001 & 0.42503 \\
\hline 2.1675 & 0.00041 & 0.00002 & 0.4335 \\
\hline 2.21 & 0.00047 & 0.00002 & 0.442 \\
\hline 2.25297 & 0.00101 & 0.00005 & 0.45059 \\
\hline 2.29563 & 0.00144 & 0.00007 & 0.45913 \\
\hline 2.33797 & 0.0017 & 0.00009 & 0.46759 \\
\hline 2.38016 & 0 & 0 & 0.47603 \\
\hline 2.42219 & -0.00015 & -0.00001 & 0.48444 \\
\hline 2.46406 & 0.00203 & 0.0001 & 0.49281 \\
\hline 2.50547 & 0.00302 & 0.00015 & 0.50109 \\
\hline 2.54719 & 0.00141 & 0.00007 & 0.50944 \\
\hline 2.58938 & 0 & 0 & 0.51788 \\
\hline 2.63172 & 0.0018 & 0.00009 & 0.52634 \\
\hline 2.67438 & 0.00304 & 0.00015 & 0.53488 \\
\hline 2.71734 & 0.0027 & 0.00014 & 0.54347 \\
\hline
\end{tabular}

\begin{tabular}{|c|c|c|c|}
\hline $\begin{array}{l}\text { Extension } \\
\text { (mm] }\end{array}$ & $\begin{array}{l}\text { Load } \\
\text { (N) }\end{array}$ & $\begin{array}{c}\text { Compressive stress } \\
\text { (MPa) }\end{array}$ & $\begin{array}{c}\text { Compressive strain } \\
\text { [mmimm })\end{array}$ \\
\hline 2.80312 & 0.00176 & 0.00009 & 0.56062 \\
\hline 2.84547 & 0.00089 & 0.00005 & 0.56909 \\
\hline 2.88766 & -0.00021 & -0.00001 & 0.57753 \\
\hline 2.93 & 0.00097 & 0.00005 & 0.586 \\
\hline 2.97234 & 0.00314 & 0.00016 & 0.59447 \\
\hline 3.01453 & 0.00405 & 0.00021 & 0.60291 \\
\hline 3.05656 & 0.00381 & 0.00019 & 0.61131 \\
\hline 3.09813 & 0.00207 & 0.00011 & 0.61963 \\
\hline 3.13969 & 0.00183 & 0.00009 & 0.62794 \\
\hline 3.18219 & 0.00403 & 0.00021 & 0.63644 \\
\hline 3.22484 & 0.00378 & 0.00019 & 0.64497 \\
\hline 3.26719 & 0.00131 & 0.00007 & 0.65344 \\
\hline 3.30969 & -0.00004 & 0 & 0.66194 \\
\hline 3.35203 & -0.00038 & -0.00002 & 0.67041 \\
\hline 3.39406 & -0.00104 & -0.00005 & 0.67881 \\
\hline 3.43594 & -0.00145 & -0.00007 & 0.68719 \\
\hline 3.47859 & 0.00251 & 0.00013 & 0.69572 \\
\hline 3.52219 & 0.0052 & 0.00027 & 0.70444 \\
\hline 3.56516 & 0.00354 & 0.00018 & 0.71303 \\
\hline 3.6075 & 0.00209 & 0.00011 & 0.7215 \\
\hline 3.64953 & 0.002 & 0.0001 & 0.72991 \\
\hline 3.69141 & 0.00221 & 0.00011 & 0.73828 \\
\hline 3.73281 & 0.00169 & 0.00009 & 0.74656 \\
\hline 3.77453 & 0.00054 & 0.00003 & 0.75491 \\
\hline 3.81687 & 0.00135 & 0.00007 & 0.76337 \\
\hline 3.85969 & 0.00108 & 0.00005 & 0.77194 \\
\hline 3.90203 & 0.00214 & 0.00011 & 0.78041 \\
\hline 3.94422 & 0.00386 & 0.0002 & 0.78884 \\
\hline 3.98688 & 0.0043 & 0.00022 & 0.79738 \\
\hline 4.02938 & 0.00446 & 0.00023 & 0.80588 \\
\hline 4.07172 & 0.00355 & 0.00018 & 0.81434 \\
\hline 4.11391 & 0.00293 & 0.00015 & 0.82278 \\
\hline 4.15641 & 0.00289 & 0.00015 & 0.83128 \\
\hline 4.19922 & 0.00246 & 0.00013 & 0.83984 \\
\hline 4.24219 & 0.00262 & 0.00013 & 0.84844 \\
\hline 4.285 & 0.00247 & 0.00013 & 0.857 \\
\hline 4.32703 & 0.00111 & 0.00006 & 0.86541 \\
\hline 4.36844 & -0.00097 & -0.00005 & 0.87369 \\
\hline 4.41031 & -0.00201 & -0.0001 & 0.88206 \\
\hline 4.45281 & 0.00137 & 0.00007 & 0.89056 \\
\hline 4.49516 & 0.00317 & 0.00016 & 0.89903 \\
\hline 4.53734 & 0.00182 & 0.00009 & 0.90747 \\
\hline 4.58 & 0.00155 & 0.00008 & 0.916 \\
\hline 4.62266 & 0.00375 & 0.00019 & 0.92453 \\
\hline 4.66516 & 0.00359 & 0.00018 & 0.93303 \\
\hline 4.70781 & 0.00151 & 0.00008 & 0.94156 \\
\hline 4.75078 & 0.00361 & 0.00018 & 0.95016 \\
\hline 4.79313 & 0.00304 & 0.00015 & 0.95863 \\
\hline 4.83484 & -0.00116 & -0.00006 & 0.96697 \\
\hline 4.87734 & -0.00055 & -0.00003 & 0.97547 \\
\hline 4.91984 & 0.00405 & 0.00021 & 0.98397 \\
\hline 4.96219 & 0.00589 & 0.0003 & 0.99244 \\
\hline 5.00344 & 0.0021 & 0.00011 & 1.00069 \\
\hline 5.045 & 0.00062 & 0.00003 & 1.009 \\
\hline 5.08734 & 0.00258 & 0.00013 & 1.01747 \\
\hline 5.13016 & 0.00373 & 0.00019 & 1.02603 \\
\hline 5.17281 & 0.00333 & 0.00017 & 1.03456 \\
\hline 5.21531 & 0.00196 & 0.0001 & 1.04306 \\
\hline 5.25797 & 0.00146 & 0.00007 & 1.05159 \\
\hline 5.30047 & 0.00175 & 0.00009 & 1.06009 \\
\hline 5.34281 & 0.00078 & 0.00004 & 1.06856 \\
\hline 5.38578 & 0.00142 & 0.00007 & 1.07716 \\
\hline 5.42844 & 0.00425 & 0.00022 & 1.08569 \\
\hline 5.47078 & 0.00469 & 0.00024 & 1.09416 \\
\hline 5.51281 & 0.00288 & 0.00015 & 1.10256 \\
\hline
\end{tabular}




\begin{tabular}{|c|c|c|c|}
\hline $\begin{array}{l}\text { Extension } \\
\text { (mm) }\end{array}$ & $\begin{array}{l}\text { Load } \\
\text { (N) }\end{array}$ & $\begin{array}{c}\text { Compressive stress } \\
\text { [MPa] }\end{array}$ & $\begin{array}{c}\text { Compressive strain } \\
{[\mathrm{mm} / \mathrm{mm}]}\end{array}$ \\
\hline 5.59594 & 0.00181 & 0.00009 & 1.11919 \\
\hline 5.63719 & 0.00294 & 0.00015 & 1.12744 \\
\hline 5.67969 & 0.00463 & 0.00024 & 1.13594 \\
\hline 5.72297 & 0.00767 & 0.00039 & 1.14459 \\
\hline 5.76531 & 0.00611 & 0.00031 & 1.15306 \\
\hline 5.80734 & 0.00197 & 0.0001 & 1.16147 \\
\hline 5.84984 & 0.00045 & 0.00002 & 1.16997 \\
\hline 5.89266 & 0.00373 & 0.00019 & 1.17853 \\
\hline 5.93516 & 0.00566 & 0.00029 & 1.18703 \\
\hline 5.97781 & 0.00215 & 0.00011 & 1.19556 \\
\hline 6.02047 & 0.0006 & 0.00003 & 1.20409 \\
\hline 6.06297 & 0.00138 & 0.00007 & 1.21259 \\
\hline 6.10531 & 0.00064 & 0.00003 & 1.22106 \\
\hline 6.1475 & -0.00065 & -0.00003 & 1.2295 \\
\hline 6.18953 & 0.00008 & 0 & 1.23791 \\
\hline 6.23125 & 0.00211 & 0.00011 & 1.24625 \\
\hline 6.27281 & 0.00276 & 0.00014 & 1.25456 \\
\hline 6.31516 & 0.00307 & 0.00016 & 1.26303 \\
\hline 6.3575 & 0.0058 & 0.0003 & 1.2715 \\
\hline 6.40016 & 0.007 & 0.00036 & 1.28003 \\
\hline 6.44297 & 0.00625 & 0.00032 & 1.28859 \\
\hline 6.48531 & 0.00372 & 0.00019 & 1.29706 \\
\hline 6.52719 & 0.00082 & 0.00004 & 1.30544 \\
\hline 6.56938 & 0.00124 & 0.00006 & 1.31388 \\
\hline 6.61234 & 0.00221 & 0.00011 & 1.32247 \\
\hline 6.65516 & 0.00369 & 0.00019 & 1.33103 \\
\hline 6.69766 & 0.00341 & 0.00017 & 1.33953 \\
\hline 6.74016 & 0.00184 & 0.00009 & 1.34803 \\
\hline 6.7825 & 0.00139 & 0.00007 & 1.3565 \\
\hline 6.82438 & 0.00126 & 0.00006 & 1.36488 \\
\hline 6.86594 & 0.00372 & 0.00019 & 1.37319 \\
\hline 6.90781 & 0.00615 & 0.00031 & 1.38156 \\
\hline 6.95016 & 0.00586 & 0.0003 & 1.39003 \\
\hline 6.9925 & 0.00485 & 0.00025 & 1.3985 \\
\hline 7.03484 & 0.00432 & 0.00022 & 1.40697 \\
\hline 70775 & 0.00368 & 0.00019 & 1.4155 \\
\hline 7.12016 & 0.00338 & 0.00017 & 1.42403 \\
\hline 7.16266 & 0.0038 & 0.00019 & 1.43253 \\
\hline 7.20516 & 0.00307 & 0.00016 & 1.44103 \\
\hline 7.24828 & 0.00178 & 0.00009 & 1.44966 \\
\hline 7.29094 & 0.00233 & 0.00012 & 1.45819 \\
\hline 7.33328 & 0.0039 & 0.0002 & 1.46666 \\
\hline 7.37563 & 0.00449 & 0.00023 & 1.47513 \\
\hline 7.41813 & 0.00286 & 0.00015 & 1.48363 \\
\hline 7.45969 & 0.00076 & 0.00004 & 1.49194 \\
\hline 7.50125 & 0.00148 & 0.00008 & 1.50025 \\
\hline 7.54359 & 0.00304 & 0.00015 & 1.50872 \\
\hline 7.58609 & 0.00627 & 0.00032 & 1.51722 \\
\hline 7.62844 & 0.00783 & 0.0004 & 1.52569 \\
\hline 7.67063 & 0.0071 & 0.00036 & 1.53413 \\
\hline 7.7125 & 0.00434 & 0.00022 & 1.5425 \\
\hline 7.75453 & 0.00185 & 0.00009 & 1.55091 \\
\hline 7.79688 & 0.00223 & 0.00011 & 1.55938 \\
\hline 7.83953 & 0.00402 & 0.0002 & 1.56791 \\
\hline 7.88234 & 0.00522 & 0.00027 & 1.57647 \\
\hline 7.92516 & 0.00474 & 0.00024 & 1.58503 \\
\hline 7.96766 & 0.00294 & 0.00015 & 1.59353 \\
\hline 8.01031 & 0.00295 & 0.00015 & 1.60206 \\
\hline 8.05234 & 0.00361 & 0.00018 & 1.61047 \\
\hline 8.09406 & 0.00376 & 0.00019 & 1.61881 \\
\hline 8.13562 & 0.00336 & 0.00017 & 1.62713 \\
\hline 8.17766 & 0.0041 & 0.00021 & 1.63553 \\
\hline 8.22 & 0.00338 & 0.00017 & 1.644 \\
\hline 8.26219 & 0.00243 & 0.00012 & 1.65244 \\
\hline 8.30469 & 0.00251 & 0.00013 & 1.66094 \\
\hline
\end{tabular}

\begin{tabular}{|c|c|c|c|}
\hline $\begin{array}{l}\text { Extension } \\
\text { (mm) }\end{array}$ & $\begin{array}{l}\text { Load } \\
\text { (N) }\end{array}$ & $\begin{array}{c}\text { Compressive stress } \\
\text { (MPa) }\end{array}$ & $\begin{array}{c}\text { Compressive strain } \\
\text { [mmimm] }\end{array}$ \\
\hline 8.39 & 0.00424 & 0.00022 & 1.678 \\
\hline 8.43219 & 0.00148 & 0.00008 & 1.68644 \\
\hline 8.47453 & 0.001 & 0.00005 & 1.69491 \\
\hline 8.51703 & 0.00278 & 0.00014 & 1.70341 \\
\hline 8.55969 & 0.00258 & 0.00013 & 1.71194 \\
\hline 8.60188 & 0.00177 & 0.00009 & 1.72038 \\
\hline 8.64422 & 0.00159 & 0.00008 & 1.72884 \\
\hline 8.68641 & 0.00004 & 0 & 1.73728 \\
\hline 8.72813 & -0.00164 & -0.00008 & 1.74563 \\
\hline 8.76984 & 0.0001 & 0 & 1.75397 \\
\hline 8.81219 & 0.004 & 0.0002 & 1.76244 \\
\hline 8.85469 & 0.00528 & 0.00027 & 1.77094 \\
\hline 8.89719 & 0.00342 & 0.00017 & 1.77944 \\
\hline 8.93969 & 0.00081 & 0.00004 & 1.78794 \\
\hline 8.9825 & 0.00143 & 0.00007 & 1.7965 \\
\hline 9.02516 & 0.00285 & 0.00014 & 1.80503 \\
\hline 9.06734 & 0.00254 & 0.00013 & 1.81347 \\
\hline 9.10969 & 0.00339 & 0.00017 & 1.82194 \\
\hline 9.15234 & 0.00498 & 0.00025 & 1.83047 \\
\hline 9.19484 & 0.00547 & 0.00028 & 1.83897 \\
\hline 9.23734 & 0.00543 & 0.00028 & 1.84747 \\
\hline 9.27984 & 0.0066 & 0.00034 & 1.85597 \\
\hline 9.32172 & 0.00665 & 0.00034 & 1.86434 \\
\hline 9.36312 & 0.00395 & 0.0002 & 1.87262 \\
\hline 9.40516 & 0.0015 & 0.00008 & 1.88103 \\
\hline 9.4475 & 0.00272 & 0.00014 & 1.8895 \\
\hline 9.49 & 0.00503 & 0.00026 & 1.898 \\
\hline 9.5325 & 0.00411 & 0.00021 & 1.9065 \\
\hline 9.575 & 0.00304 & 0.00015 & 1.915 \\
\hline 9.61781 & 0.0036 & 0.00018 & 1.92356 \\
\hline 9.66047 & 0.00166 & 0.00008 & 1.93209 \\
\hline 9.70297 & 0.00243 & 0.00012 & 1.94059 \\
\hline 9.74562 & 0.02068 & 0.00105 & 1.94912 \\
\hline 9.78844 & 0.09027 & 0.0046 & 1.95769 \\
\hline 9.83078 & 0.30215 & 0.01539 & 1.96616 \\
\hline 9.87281 & 0.78984 & 0.04023 & 1.97456 \\
\hline 9.91469 & 1.54445 & 0.07866 & 1.98294 \\
\hline 9.95656 & 2.47624 & 0.12611 & 1.99131 \\
\hline 9.99797 & 3.51118 & 0.17882 & 1.99959 \\
\hline 10.04016 & 4.60056 & 0.2343 & 2.00803 \\
\hline 10.0825 & 5.72051 & 0.29134 & 2.0165 \\
\hline 10.125 & 6.8489 & 0.34881 & 2.025 \\
\hline 10.1675 & 7.87136 & 0.40088 & 2.0335 \\
\hline 10.21016 & 8.58833 & 0.4374 & 2.04203 \\
\hline 10.25281 & 9.04562 & 0.46069 & 2.05056 \\
\hline 10.29531 & 9.34951 & 0.47617 & 2.05906 \\
\hline 10.33781 & 9.69718 & 0.49387 & 2.06756 \\
\hline 10.38047 & 10.34676 & 0.52696 & 2.07609 \\
\hline 10.42328 & 11.25739 & 0.57333 & 2.08466 \\
\hline 10.46562 & 12.34247 & 0.6286 & 2.09312 \\
\hline 10.50797 & 13.52975 & 0.68906 & 2.10159 \\
\hline 10.54969 & 14.77337 & 0.7524 & 2.10994 \\
\hline 10.59109 & 16.04027 & 0.81692 & 2.11822 \\
\hline 10.63266 & 17.31923 & 0.88206 & 2.12653 \\
\hline 10.67469 & 18.6205 & 0.94833 & 2.13494 \\
\hline 10.71719 & 19.97054 & 1.01709 & 2.14344 \\
\hline 10.75953 & 21.385 & 1.08913 & 2.15191 \\
\hline 10.80219 & 22.87406 & 1.16497 & 2.16044 \\
\hline 10.845 & 24.4376 & 1.2446 & 2.169 \\
\hline 10.88766 & 26.07967 & 1.32823 & 2.17753 \\
\hline 10.92984 & 27.7907 & 1.41537 & 2.18597 \\
\hline 10.97234 & 29.55092 & 1.50502 & 2.19447 \\
\hline 11.01516 & 31.37759 & 1.59805 & 2.20303 \\
\hline 11.05734 & 33.28081 & 1.69498 & 2.21147 \\
\hline 11.09937 & 35.24226 & 1.79487 & 2.21987 \\
\hline
\end{tabular}




\begin{tabular}{|c|c|c|c|}
\hline $\begin{array}{l}\text { Extension } \\
\text { (mm) }\end{array}$ & $\begin{array}{l}\text { Load } \\
\text { (N) }\end{array}$ & $\begin{array}{c}\text { Compressive stress } \\
\text { (MPa) }\end{array}$ & $\begin{array}{c}\text { Compressive strain } \\
\text { (mm/mm) }\end{array}$ \\
\hline 11.18359 & 39.40364 & 2.00681 & 2.23672 \\
\hline 11.22547 & 41.66018 & 2.12174 & 2.24509 \\
\hline 11.2675 & 44.03904 & 2.24289 & 2.2535 \\
\hline 11.30984 & 46.53734 & 2.37013 & 2.26197 \\
\hline 11.35234 & 49.18346 & 2.50489 & 2.27047 \\
\hline 11.39437 & 51.97981 & 2.64731 & 2.27887 \\
\hline 11.43688 & 54.91506 & 2.7968 & 2.28737 \\
\hline 11.47953 & 58.01405 & 2.95463 & 2.29591 \\
\hline 11.52234 & 61.31381 & 3.12269 & 2.30447 \\
\hline 11.56484 & 64.79343 & 3.2999 & 2.31297 \\
\hline 11.60719 & 68.43219 & 3.48522 & 2.32144 \\
\hline 11.64984 & 72.24099 & 3.6792 & 2.32997 \\
\hline 11.6925 & 76.24426 & 3.88309 & 2.3385 \\
\hline 11.73469 & 80.45405 & 4.09749 & 2.34694 \\
\hline 11.77687 & 84.88269 & 4.32304 & 2.35537 \\
\hline 11.81859 & 89.53122 & 4.55979 & 2.36372 \\
\hline 11.86 & 94.39301 & 4.8074 & 2.372 \\
\hline 11.90172 & 99.46677 & 5.0658 & 2.38034 \\
\hline 11.94406 & 104.81733 & 5.3383 & 2.38881 \\
\hline 11.98641 & 110.50576 & 5.62801 & 2.39728 \\
\hline 12.02844 & 116.52595 & 5.93462 & 2.40569 \\
\hline 12.07109 & 122.87875 & 6.25816 & 2.41422 \\
\hline 12.11422 & 129.61217 & 6.60109 & 2.42284 \\
\hline 12.15672 & 136.7751 & 6.9659 & 2.43134 \\
\hline 12.19875 & 144.31984 & 7.35015 & 2.43975 \\
\hline 12.24141 & 152.21959 & 7.75248 & 2.44828 \\
\hline 12.28422 & 160.63715 & 8.18118 & 2.45684 \\
\hline 12.32672 & 169.65593 & 8.64051 & 2.46534 \\
\hline 12.36875 & 179.22391 & 9.1278 & 2.47375 \\
\hline 12.41078 & 189.33582 & 9.64279 & 2.48216 \\
\hline 12.4525 & 200.08844 & 10.19042 & 2.4905 \\
\hline 12.49375 & 211.56204 & 10.77477 & 2.49875 \\
\hline 12.53516 & 223.82738 & 11.39943 & 2.50703 \\
\hline 12.57734 & 237.09828 & 12.07532 & 2.51547 \\
\hline 12.61922 & 251.5887 & 12.81331 & 2.52384 \\
\hline 12.66141 & 267.29239 & 13.61309 & 2.53228 \\
\hline 12.70359 & 284.2142 & 14.47491 & 2.54072 \\
\hline 12.74609 & 302.41537 & 15.40189 & 2.54922 \\
\hline 12.78859 & 321.95972 & 16.39727 & 2.55772 \\
\hline 12.83078 & 342.86417 & 17.46193 & 2.56616 \\
\hline 12.87281 & 365.14426 & 18.59664 & 2.57456 \\
\hline 12.91531 & 388.95563 & 19.80935 & 2.58306 \\
\hline 12.95766 & 414.55957 & 21.11335 & 2.59153 \\
\hline 12.99953 & 442.24133 & 22.52317 & 2.59991 \\
\hline 13.01594 & 453.91934 & 23.11792 & 2.60319 \\
\hline
\end{tabular}


Table D.6 ODM-Soaked Scaffolds Without Osteoblasts Specimen 2 Raw Compression Data

\begin{tabular}{|c|c|c|c|}
\hline $\begin{array}{l}\text { Extension } \\
\text { (mm) }\end{array}$ & $\begin{array}{c}\text { Load } \\
\text { [N] }\end{array}$ & $\begin{array}{c}\text { Compressive stress } \\
\text { (MPa] }\end{array}$ & $\begin{array}{c}\text { Compressive strain } \\
\text { [mmimm] }\end{array}$ \\
\hline 0 & 0.00226 & 0.00012 & 0 \\
\hline 0.00281 & 0.00306 & 0.00016 & 0.00056 \\
\hline 0.02375 & 0.01983 & 0.00101 & 0.00475 \\
\hline 0.06563 & 0.0574 & 0.00292 & 0.01313 \\
\hline 0.11188 & 0.07837 & 0.00399 & 0.02238 \\
\hline 0.15344 & 0.06176 & 0.00315 & 0.03069 \\
\hline 0.19625 & 0.03383 & 0.00172 & 0.03925 \\
\hline 0.24484 & 0.0218 & 0.00111 & 0.04897 \\
\hline 0.29563 & 0.02 & 0.00102 & 0.05913 \\
\hline 0.34406 & 0.01198 & 0.00061 & 0.06881 \\
\hline 0.39109 & 0.00047 & 0.00002 & 0.07822 \\
\hline 0.43828 & -0.00455 & -0.00023 & 0.08766 \\
\hline 0.48547 & -0.00103 & -0.00005 & 0.09709 \\
\hline 0.53203 & 0.00097 & 0.00005 & 0.10641 \\
\hline 0.57734 & -0.00239 & -0.00012 & 0.11547 \\
\hline 0.62172 & -0.00633 & -0.00032 & 0.12434 \\
\hline 0.66641 & -0.00613 & -0.00031 & 0.13328 \\
\hline 0.71141 & -0.00249 & $\begin{array}{l}-0.00013 \\
\end{array}$ & 0.14228 \\
\hline 0.75594 & 0.00034 & 0.00002 & 0.15119 \\
\hline 0.79969 & 0.00059 & 0.00003 & 0.15994 \\
\hline 0.84328 & 0.00029 & 0.00001 & 0.16866 \\
\hline 0.88703 & -0.00136 & -0.00007 & 0.17741 \\
\hline 0.93062 & -0.00154 & -0.00008 & 0.18612 \\
\hline 0.97438 & 0.00061 & 0.00003 & 0.19488 \\
\hline 1.01828 & 0.00182 & 0.00009 & 0.20366 \\
\hline 1.06203 & 0.00092 & 0.00005 & 0.21241 \\
\hline 1.10531 & 0.0002 & 0.00001 & 0.22106 \\
\hline 1.14812 & 0.00105 & 0.00005 & 0.22962 \\
\hline 1.19062 & 0.00006 & 0 & 0.23812 \\
\hline 1.23266 & -0.00299 & -0.00015 & 0.24653 \\
\hline 1.275 & -0.00345 & -0.00018 & 0.255 \\
\hline 1.3175 & 0.00039 & 0.00002 & 0.2635 \\
\hline 1.36016 & 0.00305 & 0.00016 & 0.27203 \\
\hline 1.40281 & 0.0037 & 0.00019 & 0.28056 \\
\hline 1.44547 & 0.00301 & 0.00015 & 0.28909 \\
\hline 1.48813 & 0.00445 & 0.00023 & 0.29763 \\
\hline 1.53078 & 0.00496 & 0.00025 & 0.30616 \\
\hline 1.57359 & 0.00295 & 0.00015 & 0.31472 \\
\hline 1.61688 & 0.00065 & 0.00003 & 0.32338 \\
\hline 1.65969 & -0.00157 & $\begin{array}{c}-0.00008 \\
\end{array}$ & 0.33194 \\
\hline 1.70234 & -0.00204 & -0.0001 & 0.34047 \\
\hline 1.74453 & -0.0025 & -0.00013 & 0.34891 \\
\hline 1.78688 & 0.0004 & 0.00002 & 0.35737 \\
\hline 1.82875 & 0.00237 & 0.00012 & 0.36575 \\
\hline 1.87031 & 0.00052 & 0.00003 & 0.37406 \\
\hline 1.91234 & -0.00013 & -0.00001 & 0.38247 \\
\hline 1.95453 & 0.00349 & 0.00018 & 0.39091 \\
\hline 1.99687 & 0.00791 & 0.0004 & 0.39937 \\
\hline 2.03938 & 0.00703 & 0.00036 & 0.40788 \\
\hline 2.08219 & 0.00387 & 0.0002 & 0.41644 \\
\hline 2.125 & 0.00267 & 0.00014 & 0.425 \\
\hline 2.1675 & 0.0026 & 0.00013 & 0.4335 \\
\hline 2.21016 & 0.00104 & 0.00005 & 0.44203 \\
\hline 2.25313 & 0.00026 & 0.00001 & 0.45063 \\
\hline 2.29562 & 0.00185 & 0.00009 & 0.45913 \\
\hline 2.33781 & 0.00019 & 0.00001 & 0.46756 \\
\hline 2.37984 & -0.00115 & -0.00006 & 0.47597 \\
\hline 2.42203 & 0.00111 & 0.00006 & 0.48441 \\
\hline 2.46391 & 0.00266 & 0.00014 & 0.49278 \\
\hline 2.50547 & 0.00311 & 0.00016 & 0.50109 \\
\hline 2.5475 & 0.00504 & 0.00026 & 0.5095 \\
\hline 2.59 & 0.00611 & 0.00031 & 0.518 \\
\hline 2.63266 & 0.00613 & 0.00031 & 0.52653 \\
\hline 2.675 & 0.00532 & 0.00027 & 0.535 \\
\hline 2.7175 & 0.00207 & 0.00011 & 0.5435 \\
\hline
\end{tabular}

\begin{tabular}{|c|c|c|c|}
\hline $\begin{array}{c}\text { Extension } \\
\text { (mm) }\end{array}$ & $\begin{array}{c}\text { Load } \\
\text { (N) }\end{array}$ & $\begin{array}{c}\text { Compressive stress } \\
\text { (MPa] }\end{array}$ & $\begin{array}{c}\text { Compressive strain } \\
\text { (mm/mm) }\end{array}$ \\
\hline 2.80281 & -0.00124 & -0.00006 & 0.56056 \\
\hline 2.84516 & -0.00036 & -0.00002 & 0.56903 \\
\hline 2.8875 & -0.00094 & -0.00005 & 0.5775 \\
\hline 2.93 & 0.0005 & 0.00003 & 0.586 \\
\hline 2.97234 & 0.00351 & 0.00018 & 0.59447 \\
\hline 3.01453 & 0.00424 & 0.00022 & 0.60291 \\
\hline 3.05625 & 0.00176 & 0.00009 & 0.61125 \\
\hline 3.0975 & -0.00126 & -0.00006 & 0.6195 \\
\hline 3.13906 & -0.00034 & -0.00002 & 0.62781 \\
\hline 3.18172 & 0.00313 & 0.00016 & 0.63634 \\
\hline 3.22438 & 0.0045 & 0.00023 & 0.64487 \\
\hline 3.26688 & 0.00662 & 0.00034 & 0.65338 \\
\hline 3.30953 & 0.00748 & 0.00038 & 0.66191 \\
\hline 3.35219 & 0.00549 & 0.00028 & 0.67044 \\
\hline 3.39453 & 0.00155 & 0.00008 & 0.67891 \\
\hline 3.43641 & 0.00171 & 0.00009 & 0.68728 \\
\hline 3.47875 & 0.00525 & 0.00027 & 0.69575 \\
\hline 3.52219 & 0.0057 & 0.00029 & 0.70444 \\
\hline 3.56516 & 0.00313 & 0.00016 & 0.71303 \\
\hline 3.60766 & 0.0009 & 0.00005 & 0.72153 \\
\hline 3.64984 & 0.00079 & 0.00004 & 0.72997 \\
\hline 3.69172 & 0.00106 & 0.00005 & 0.73834 \\
\hline 3.73328 & -0.00008 & 0 & 0.74666 \\
\hline 3.77516 & 0.00048 & 0.00002 & 0.75503 \\
\hline 3.81703 & 0.00038 & 0.00002 & 0.76341 \\
\hline 3.85922 & -0.00014 & -0.00001 & 0.77184 \\
\hline 3.90156 & 0.00122 & 0.00006 & 0.78031 \\
\hline 3.94406 & 0.00496 & 0.00025 & 0.78881 \\
\hline 3.98656 & 0.00632 & 0.00032 & 0.79731 \\
\hline 4.02906 & 0.00259 & 0.00013 & 0.80581 \\
\hline 4.07203 & 0.00196 & 0.0001 & 0.81441 \\
\hline 4.115 & 0.00529 & 0.00027 & 0.823 \\
\hline 4.15766 & 0.00418 & 0.00021 & 0.83153 \\
\hline 4.19984 & 0.00103 & 0.00005 & 0.83997 \\
\hline 4.24234 & 0.00171 & 0.00009 & 0.84847 \\
\hline 4.285 & 0.00386 & 0.0002 & 0.857 \\
\hline 4.32687 & 0.00142 & 0.00007 & 0.86537 \\
\hline 4.36797 & -0.00195 & -0.0001 & 0.87359 \\
\hline 4.40984 & -0.00002 & 0 & 0.88197 \\
\hline 4.45266 & 0.00532 & 0.00027 & 0.89053 \\
\hline 4.49516 & 0.00636 & 0.00032 & 0.89903 \\
\hline 4.5375 & 0.00379 & 0.00019 & 0.9075 \\
\hline 4.57984 & 0.00226 & 0.00011 & 0.91597 \\
\hline 4.62234 & 0.00278 & 0.00014 & 0.92447 \\
\hline 4.66422 & 0.00267 & 0.00014 & 0.93284 \\
\hline 4.70656 & 0.00368 & 0.00019 & 0.94131 \\
\hline 4.75 & 0.00616 & 0.00031 & 0.95 \\
\hline 4.79359 & 0.00673 & 0.00034 & 0.95872 \\
\hline 4.83609 & 0.00419 & 0.00021 & 0.96722 \\
\hline 4.87812 & 0.00115 & 0.00006 & 0.97562 \\
\hline 4.92016 & 0.00147 & 0.00007 & 0.98403 \\
\hline 4.96156 & 0.00152 & 0.00008 & 0.99231 \\
\hline 5.00297 & 0.00021 & 0.00001 & 1.00059 \\
\hline 5.045 & 0.00071 & 0.00004 & 1.009 \\
\hline 5.08797 & 0.00425 & 0.00022 & 1.01759 \\
\hline 5.13047 & 0.00631 & 0.00032 & 1.02609 \\
\hline 5.17281 & 0.00549 & 0.00028 & 1.03456 \\
\hline 5.21531 & 0.00366 & 0.00019 & 1.04306 \\
\hline 5.25797 & 0.00124 & 0.00006 & 1.05159 \\
\hline 5.30047 & -0.00046 & -0.00002 & 1.06009 \\
\hline 5.34281 & -0.00014 & $\begin{array}{l}-0.00001 \\
\end{array}$ & 1.06856 \\
\hline 5.38531 & 0.00217 & 0.00011 & 1.07706 \\
\hline 5.42766 & 0.00345 & 0.00018 & 1.08553 \\
\hline 5.47 & 0.00149 & 0.00008 & 1.094 \\
\hline 5.51297 & 0.00025 & 0.00001 & 1.10259 \\
\hline
\end{tabular}




\begin{tabular}{|c|c|c|c|}
\hline $\begin{array}{c}\text { Extension } \\
\text { (mm) }\end{array}$ & $\begin{array}{l}\text { Load } \\
\text { (N) }\end{array}$ & $\begin{array}{c}\text { Compressive stress } \\
\text { (MPa] }\end{array}$ & $\begin{array}{c}\text { Compressive strain } \\
\text { (mm/mm) }\end{array}$ \\
\hline 5.59688 & 0.00175 & 0.00009 & 1.11937 \\
\hline 5.63797 & 0.00097 & 0.00005 & 1.12759 \\
\hline 5.68 & 0.00363 & 0.00018 & 1.136 \\
\hline 5.72266 & 0.00693 & 0.00035 & 1.14453 \\
\hline 5.765 & 0.00712 & 0.00036 & 1.153 \\
\hline 5.80703 & 0.00448 & 0.00023 & 1.16141 \\
\hline 5.84953 & 0.00373 & 0.00019 & 1.16991 \\
\hline 5.8925 & 0.00521 & 0.00027 & 1.1785 \\
\hline 5.93516 & 0.0069 & 0.00035 & 1.18703 \\
\hline 5.9775 & 0.00599 & 0.00031 & 1.1955 \\
\hline 6.02031 & 0.00307 & 0.00016 & 1.20406 \\
\hline 6.06297 & 0.00252 & 0.00013 & 1.21259 \\
\hline 6.105 & 0.00287 & 0.00015 & 1.221 \\
\hline 6.14703 & 0.00166 & 0.00008 & 1.22941 \\
\hline 6.18922 & 0.00048 & 0.00002 & 1.23784 \\
\hline 6.23125 & 0.00076 & 0.00004 & 1.24625 \\
\hline 6.27344 & 0.00261 & 0.00013 & 1.25469 \\
\hline 6.31594 & 0.00418 & 0.00021 & 1.26319 \\
\hline 6.35812 & 0.0055 & 0.00028 & 1.27162 \\
\hline 6.40016 & 0.00636 & 0.00032 & 1.28003 \\
\hline 6.44266 & 0.00554 & 0.00028 & 1.28853 \\
\hline 6.48469 & 0.00357 & 0.00018 & 1.29694 \\
\hline 6.52656 & 0.00195 & 0.0001 & 1.30531 \\
\hline 6.56891 & 0.00297 & 0.00015 & 1.31378 \\
\hline 6.61203 & 0.00515 & 0.00026 & 1.32241 \\
\hline 6.65531 & 0.00549 & 0.00028 & 1.33106 \\
\hline 6.69781 & 0.00461 & 0.00023 & 1.33956 \\
\hline 6.74016 & 0.00272 & 0.00014 & 1.34803 \\
\hline 6.78266 & 0.00198 & 0.0001 & 1.35653 \\
\hline 6.82469 & 0.00261 & 0.00013 & 1.36494 \\
\hline 6.86609 & 0.00329 & 0.00017 & 1.37322 \\
\hline 6.90781 & 0.00273 & 0.00014 & 1.38156 \\
\hline 6.95031 & 0.00263 & 0.00013 & 1.39006 \\
\hline 6.9925 & 0.004 & 0.0002 & 1.3985 \\
\hline 7.03484 & 0.00517 & 0.00026 & 1.40697 \\
\hline 7.07734 & 0.00456 & 0.00023 & 1.41547 \\
\hline 7.12016 & 0.00561 & 0.00029 & 1.42403 \\
\hline 7.16281 & 0.00649 & 0.00033 & 1.43256 \\
\hline 7.20547 & 0.00588 & 0.0003 & 1.44109 \\
\hline 7.24828 & 0.00633 & 0.00032 & 1.44966 \\
\hline 7.29094 & 0.00642 & 0.00033 & 1.45819 \\
\hline 7.33313 & 0.00496 & 0.00025 & 1.46663 \\
\hline 7.37531 & 0.00368 & 0.00019 & 1.47506 \\
\hline 7.41781 & 0.004 & 0.0002 & 1.48356 \\
\hline 7.45984 & 0.00207 & 0.00011 & 1.49197 \\
\hline 7.50156 & 0.0009 & 0.00005 & 1.50031 \\
\hline 7.54375 & 0.00322 & 0.00016 & 1.50875 \\
\hline 7.58594 & 0.00562 & 0.00029 & 1.51719 \\
\hline 7.62828 & 0.00689 & 0.00035 & 1.52566 \\
\hline 7.67062 & 0.00733 & 0.00037 & 1.53412 \\
\hline 7.71281 & 0.0067 & 0.00034 & 1.54256 \\
\hline 7.75484 & 0.0039 & 0.0002 & 1.55097 \\
\hline 7.79688 & 0.00361 & 0.00018 & 1.55938 \\
\hline 7.83953 & 0.0067 & 0.00034 & 1.56791 \\
\hline 7.88281 & 0.0066 & 0.00034 & 1.57656 \\
\hline 7.92547 & 0.00377 & 0.00019 & 1.58509 \\
\hline 7.96781 & 0.00177 & 0.00009 & 1.59356 \\
\hline 8.01 & 0.00224 & 0.00011 & 1.602 \\
\hline 8.05219 & 0.00352 & 0.00018 & 1.61044 \\
\hline 8.09375 & 0.00376 & 0.00019 & 1.61875 \\
\hline 8.13516 & 0.0042 & 0.00021 & 1.62703 \\
\hline 8.17719 & 0.006 & 0.00031 & 1.63544 \\
\hline 8.21938 & 0.0063 & 0.00032 & 1.64388 \\
\hline 8.26203 & 0.00746 & 0.00038 & 1.65241 \\
\hline 8.30469 & 0.00767 & 0.00039 & 1.66094 \\
\hline
\end{tabular}

\begin{tabular}{|c|c|c|c|}
\hline $\begin{array}{l}\text { Extension } \\
\text { (mm) }\end{array}$ & $\begin{array}{c}\text { Load } \\
\text { (N) }\end{array}$ & \begin{tabular}{|c|} 
Compressive stress \\
(MPa)
\end{tabular} & $\begin{array}{c}\text { Compressive strain } \\
\text { (mm/mm) }\end{array}$ \\
\hline 8.38984 & 0.00498 & 0.00025 & 1.67797 \\
\hline 8.43203 & 0.00148 & 0.00008 & 1.68641 \\
\hline 8.47422 & 0.00219 & 0.00011 & 1.69484 \\
\hline 8.51688 & 0.00431 & 0.00022 & 1.70338 \\
\hline 8.55937 & 0.00413 & 0.00021 & 1.71187 \\
\hline 8.60188 & 0.00354 & 0.00018 & 1.72038 \\
\hline 8.64437 & 0.00407 & 0.00021 & 1.72888 \\
\hline 8.68672 & 0.0052 & 0.00027 & 1.73734 \\
\hline 8.72859 & 0.0038 & 0.00019 & 1.74572 \\
\hline 8.77031 & 0.00283 & 0.00014 & 1.75406 \\
\hline 8.8125 & 0.00593 & 0.0003 & 1.7625 \\
\hline 8.85484 & 0.0086 & 0.00044 & 1.77097 \\
\hline 8.89703 & 0.00672 & 0.00034 & 1.77941 \\
\hline 8.93953 & 0.00418 & 0.00021 & 1.78791 \\
\hline 8.98219 & 0.0043 & 0.00022 & 1.79644 \\
\hline 9.02484 & 0.00394 & 0.0002 & 1.80497 \\
\hline 9.06719 & 0.00291 & 0.00015 & 1.81344 \\
\hline 9.11 & 0.00321 & 0.00016 & 1.822 \\
\hline 9.15266 & 0.00372 & 0.00019 & 1.83053 \\
\hline 9.19516 & 0.00163 & 0.00008 & 1.83903 \\
\hline 9.2375 & 0.00148 & 0.00008 & 1.8475 \\
\hline 9.27984 & 0.00319 & 0.00016 & 1.85597 \\
\hline 9.32187 & 0.00461 & 0.00023 & 1.86437 \\
\hline 9.36328 & 0.00306 & 0.00016 & 1.87266 \\
\hline 9.405 & 0.0033 & 0.00017 & 1.881 \\
\hline 9.44734 & 0.00613 & 0.00031 & 1.88947 \\
\hline 9.48984 & 0.00806 & 0.00041 & 1.89797 \\
\hline 9.53219 & 0.00849 & 0.00043 & 1.90644 \\
\hline 9.575 & 0.00743 & 0.00038 & 1.915 \\
\hline 9.61781 & 0.00666 & 0.00034 & 1.92356 \\
\hline 9.66031 & 0.00637 & 0.00032 & 1.93206 \\
\hline 9.70281 & 0.00573 & 0.00029 & 1.94056 \\
\hline 9.74562 & 0.00476 & 0.00024 & 1.94912 \\
\hline 9.78828 & 0.00545 & 0.00028 & 1.95766 \\
\hline 9.83078 & 0.00679 & 0.00035 & 1.96616 \\
\hline 9.87281 & 0.00407 & 0.00021 & 1.97456 \\
\hline 9.91484 & 0.00208 & 0.00011 & 1.98297 \\
\hline 9.95656 & 0.00413 & 0.00021 & 1.99131 \\
\hline 9.99797 & 0.0062 & 0.00032 & 1.99959 \\
\hline 10.04016 & 0.00676 & 0.00034 & 2.00803 \\
\hline 10.08266 & 0.00667 & 0.00034 & 2.01653 \\
\hline 10.12516 & 0.00731 & 0.00037 & 2.02503 \\
\hline 10.1675 & 0.00717 & 0.00037 & 2.0335 \\
\hline 10.21 & 0.00629 & 0.00032 & 2.042 \\
\hline 10.25281 & 0.00662 & 0.00034 & 2.05056 \\
\hline 10.29547 & 0.00779 & 0.0004 & 2.05909 \\
\hline 10.33844 & 0.00704 & 0.00036 & 2.06769 \\
\hline 10.38125 & 0.00415 & 0.00021 & 2.07625 \\
\hline 10.42375 & 0.00409 & 0.00021 & 2.08475 \\
\hline 10.46594 & 0.00726 & 0.00037 & 2.09319 \\
\hline 10.50813 & 0.0167 & 0.00085 & 2.10162 \\
\hline 10.54984 & 0.05669 & 0.00289 & 2.10997 \\
\hline 10.59125 & 0.16102 & 0.0082 & 2.11825 \\
\hline 10.63281 & 0.3502 & 0.01784 & 2.12656 \\
\hline 10.675 & 0.62739 & 0.03195 & 2.135 \\
\hline 10.71734 & 0.99892 & 0.05087 & 2.14347 \\
\hline 10.75969 & 1.45666 & 0.07419 & 2.15194 \\
\hline 10.80203 & 1.98727 & 0.10121 & 2.16041 \\
\hline 10.845 & 2.58471 & 0.13164 & 2.169 \\
\hline 10.88797 & 3.24119 & 0.16507 & 2.17759 \\
\hline 10.93047 & 3.9497 & 0.20116 & 2.18609 \\
\hline 10.97281 & 4.70449 & 0.2396 & 2.19456 \\
\hline 11.01547 & 5.51703 & 0.28098 & 2.20309 \\
\hline 11.05797 & 6.39936 & 0.32592 & 2.21159 \\
\hline 11.10031 & 7.35254 & 0.37446 & 2.22006 \\
\hline
\end{tabular}




\begin{tabular}{|c|c|c|c|}
\hline $\begin{array}{l}\text { Extension } \\
\text { (mm) }\end{array}$ & $\begin{array}{l}\text { Load } \\
\text { (N) }\end{array}$ & $\begin{array}{c}\text { Compressive stress } \\
\text { (MPa] }\end{array}$ & $\begin{array}{c}\text { Compressive strain } \\
{[\mathrm{mm} / \mathrm{mm})}\end{array}$ \\
\hline 11.05797 & 6.39936 & 0.32592 & 2.21159 \\
\hline 11.10031 & 7.35254 & 0.37446 & 2.22006 \\
\hline 11.14234 & 8.37009 & 0.42629 & 2.22847 \\
\hline 11.18422 & 9.36096 & 0.47675 & 2.23684 \\
\hline 11.22578 & 10.10955 & 0.51488 & 2.24516 \\
\hline 11.26781 & 10.59497 & 0.5396 & 2.25356 \\
\hline 11.31016 & 10.94347 & 0.55735 & 2.26203 \\
\hline 11.35266 & 11.24294 & 0.5726 & 2.27053 \\
\hline 11.39484 & 11.85497 & 0.60377 & 2.27897 \\
\hline 11.43719 & 12.85535 & 0.65472 & 2.28744 \\
\hline 11.47984 & 14.14203 & 0.72025 & 2.29597 \\
\hline 11.5225 & 15.63755 & 0.79641 & 2.3045 \\
\hline 11.565 & 17.28265 & 0.8802 & 2.313 \\
\hline 11.6075 & 19.0455 & 0.96998 & 2.3215 \\
\hline 11.65016 & 20.9123 & 1.06505 & 2.33003 \\
\hline 11.69266 & 22.8827 & 1.16541 & 2.33853 \\
\hline 11.73516 & 24.96626 & 1.27152 & 2.34703 \\
\hline 11.77766 & 27.18876 & 1.38471 & 2.35553 \\
\hline 11.81969 & 29.58528 & 1.50677 & 2.36394 \\
\hline 11.86094 & 32.15771 & 1.63778 & 2.37219 \\
\hline 11.90266 & 34.8992 & 1.7774 & 2.38053 \\
\hline 11.94484 & 37.83553 & 1.92695 & 2.38897 \\
\hline 11.98734 & 40.994 & 2.08781 & 2.39747 \\
\hline 12.02953 & 44.38172 & 2.26034 & 2.40591 \\
\hline 12.07188 & 48.00283 & 2.44476 & 2.41438 \\
\hline 12.11484 & 51.8949 & 2.64299 & 2.42297 \\
\hline 12.1575 & 56.11897 & 2.85812 & 2.4315 \\
\hline 12.19969 & 60.67152 & 3.08997 & 2.43994 \\
\hline 12.24234 & 65.53982 & 3.33792 & 2.44847 \\
\hline 12.285 & 70.78289 & 3.60494 & 2.457 \\
\hline 12.32734 & 76.44941 & 3.89354 & 2.46547 \\
\hline 12.36938 & 82.53914 & 4.20368 & 2.47388 \\
\hline 12.41156 & 89.05612 & 4.53559 & 2.48231 \\
\hline 12.45344 & 96.03597 & 4.89107 & 2.49069 \\
\hline 12.49469 & 103.48216 & 5.2703 & 2.49894 \\
\hline 12.53641 & 111.4131 & 5.67422 & 2.50728 \\
\hline 12.57859 & 119.98801 & 6.11094 & 2.51572 \\
\hline 12.62094 & 129.36642 & 6.58858 & 2.52419 \\
\hline 12.66281 & 139.57187 & 7.10834 & 2.53256 \\
\hline 12.70516 & 150.61588 & 7.6708 & 2.54103 \\
\hline 12.74766 & 162.56374 & 8.2793 & 2.54953 \\
\hline 12.79016 & 175.47987 & 8.93712 & 2.55803 \\
\hline 12.8325 & 189.35252 & 9.64364 & 2.5665 \\
\hline 12.875 & 204.18768 & 10.39919 & 2.575 \\
\hline 12.9175 & 220.09558 & 11.20938 & 2.5835 \\
\hline 12.95969 & 237.17447 & 12.0792 & 2.59194 \\
\hline 13.00156 & 255.51051 & 13.01304 & 2.60031 \\
\hline 13.04344 & 275.1145 & 14.01147 & 2.60869 \\
\hline 13.08469 & 296.04623 & 15.07751 & 2.61694 \\
\hline 13.12578 & 318.46317 & 16.2192 & 2.62516 \\
\hline 13.16734 & 342.4512 & 17.4409 & 2.63347 \\
\hline 13.20922 & 368.2962 & 18.75717 & 2.64184 \\
\hline 13.25125 & 396.3295 & 20.18489 & 2.65025 \\
\hline 13.29297 & 426.74759 & 21.73408 & 2.65859 \\
\hline 13.32656 & 452.83224 & 23.06256 & 2.66531 \\
\hline
\end{tabular}


Table D.7ODM-Soaked Scaffolds Without Osteoblasts Specimen 3 Raw Compression Data

\begin{tabular}{|c|c|c|c|}
\hline $\begin{array}{c}\text { Extension } \\
\text { (mm) }\end{array}$ & $\begin{array}{c}\text { Load } \\
\text { (N) }\end{array}$ & $\begin{array}{c}\text { Compressive stress } \\
\text { (MPa] }\end{array}$ & $\begin{array}{c}\text { Compressive strain } \\
{[\mathrm{mm} / \mathrm{mm}]}\end{array}$ \\
\hline 0 & -0.00038 & -0.00002 & 0 \\
\hline 0.00281 & 0.00139 & 0.00007 & 0.00056 \\
\hline 0.02375 & 0.01869 & 0.00095 & 0.00475 \\
\hline 0.06563 & 0.05873 & 0.00299 & 0.01313 \\
\hline 0.11156 & 0.08056 & 0.0041 & 0.02231 \\
\hline 0.15313 & 0.06148 & 0.00313 & 0.03063 \\
\hline 0.19594 & 0.03287 & 0.00167 & 0.03919 \\
\hline 0.24453 & 0.02319 & 0.00118 & 0.04891 \\
\hline 0.29531 & 0.02331 & 0.00119 & 0.05906 \\
\hline 0.34391 & 0.01398 & 0.00071 & 0.06878 \\
\hline 0.39094 & 0.00091 & 0.00005 & 0.07819 \\
\hline 0.43797 & -0.00352 & -0.00018 & 0.08759 \\
\hline 0.48531 & -0.00234 & -0.00012 & 0.09706 \\
\hline 0.53203 & -0.00189 & -0.0001 & 0.10641 \\
\hline 0.57703 & -0.00129 & -0.00007 & 0.11541 \\
\hline 0.62141 & -0.00184 & -0.00009 & 0.12428 \\
\hline 0.66625 & -0.00284 & -0.00014 & 0.13325 \\
\hline 0.71141 & -0.00033 & -0.00002 & 0.14228 \\
\hline 0.75594 & 0.00321 & 0.00016 & 0.15119 \\
\hline 0.79984 & 0.00419 & 0.00021 & 0.15997 \\
\hline 0.84375 & 0.00327 & 0.00017 & 0.16875 \\
\hline 0.8875 & 0.00194 & 0.0001 & 0.1775 \\
\hline 0.93078 & 0.00022 & 0.00001 & 0.18616 \\
\hline 0.97438 & -0.00053 & -0.00003 & 0.19488 \\
\hline 1.01828 & 0.00138 & 0.00007 & 0.20366 \\
\hline 1.06203 & 0.00388 & 0.0002 & 0.21241 \\
\hline 1.10531 & 0.0042 & 0.00021 & 0.22106 \\
\hline 1.14797 & 0.00153 & 0.00008 & 0.22959 \\
\hline 1.19031 & -0.00089 & -0.00005 & 0.23806 \\
\hline 1.23234 & -0.00008 & 0 & 0.24647 \\
\hline 1.27469 & 0.00289 & 0.00015 & 0.25494 \\
\hline 1.31719 & 0.0031 & 0.00016 & 0.26344 \\
\hline 1.35984 & 0.00111 & 0.00006 & 0.27197 \\
\hline 1.4025 & 0.00163 & 0.00008 & 0.2805 \\
\hline 1.44531 & 0.00402 & 0.0002 & 0.28906 \\
\hline 1.48813 & 0.00345 & 0.00018 & 0.29763 \\
\hline 1.53094 & 0.00137 & 0.00007 & 0.30619 \\
\hline 1.57391 & 0.00164 & 0.00008 & 0.31478 \\
\hline 1.61688 & 0.00248 & 0.00013 & 0.32338 \\
\hline 1.65984 & 0.00247 & 0.00013 & 0.33197 \\
\hline 1.70234 & 0.00239 & 0.00012 & 0.34047 \\
\hline 1.74469 & 0.00451 & 0.00023 & 0.34894 \\
\hline 1.78703 & 0.005 & 0.00025 & 0.35741 \\
\hline 1.82906 & 0.00173 & 0.00009 & 0.36581 \\
\hline 1.87047 & 0.00029 & 0.00001 & 0.37409 \\
\hline 1.91234 & 0.00067 & 0.00003 & 0.38247 \\
\hline 1.95469 & 0.00253 & 0.00013 & 0.39094 \\
\hline 1.99703 & 0.00434 & 0.00022 & 0.39941 \\
\hline 2.03938 & 0.00561 & 0.00029 & 0.40788 \\
\hline 2.08188 & 0.00595 & 0.0003 & 0.41638 \\
\hline 2.12453 & 0.00444 & 0.00023 & 0.42491 \\
\hline 2.16703 & 0.0037 & 0.00019 & 0.43341 \\
\hline 2.20984 & 0.00321 & 0.00016 & 0.44197 \\
\hline 2.25313 & 0.00406 & 0.00021 & 0.45063 \\
\hline 2.29578 & 0.0042 & 0.00021 & 0.45916 \\
\hline 2.33813 & 0.00125 & 0.00006 & 0.46763 \\
\hline 2.38031 & 0.00049 & 0.00003 & 0.47606 \\
\hline 2.4225 & 0.00267 & 0.00014 & 0.4845 \\
\hline 2.46422 & 0.00435 & 0.00022 & 0.49284 \\
\hline 2.50563 & 0.00398 & 0.0002 & 0.50113 \\
\hline 2.54766 & 0.00354 & 0.00018 & 0.50953 \\
\hline 2.59031 & 0.00475 & 0.00024 & 0.51806 \\
\hline 2.63281 & 0.00357 & 0.00018 & 0.52656 \\
\hline 2.675 & 0.00151 & 0.00008 & 0.535 \\
\hline 2.71766 & 0.0022 & 0.00011 & 0.54353 \\
\hline
\end{tabular}

\begin{tabular}{|c|c|c|c|}
\hline $\begin{array}{l}\text { Extension } \\
\text { (mm) }\end{array}$ & $\begin{array}{l}\text { Load } \\
\text { (N) }\end{array}$ & \begin{tabular}{|c|}
$\begin{array}{c}\text { Compressive stress } \\
\text { (MPa) }\end{array}$ \\
\end{tabular} & $\begin{array}{c}\text { Compressive strain } \\
\text { [mm/mm] }\end{array}$ \\
\hline 2.8025 & 0.00123 & 0.00006 & 0.5605 \\
\hline 2.84484 & -0.00022 & -0.00001 & 0.56897 \\
\hline 2.88734 & 0.00256 & 0.00013 & 0.57747 \\
\hline 2.92969 & 0.00461 & 0.00023 & 0.58594 \\
\hline 2.97188 & 0.00295 & 0.00015 & 0.59438 \\
\hline 3.01406 & 0.00281 & 0.00014 & 0.60281 \\
\hline 3.05625 & 0.00319 & 0.00016 & 0.61125 \\
\hline 3.09781 & 0.00188 & 0.0001 & 0.61956 \\
\hline 3.13984 & 0.00136 & 0.00007 & 0.62797 \\
\hline 3.1825 & 0.00421 & 0.00021 & 0.6365 \\
\hline 3.22484 & 0.00707 & 0.00036 & 0.64497 \\
\hline 3.26672 & 0.00513 & 0.00026 & 0.65334 \\
\hline 3.30922 & 0.00255 & 0.00013 & 0.66184 \\
\hline 3.35219 & 0.00434 & 0.00022 & 0.67044 \\
\hline 3.39469 & 0.0059 & 0.0003 & 0.67894 \\
\hline 3.43656 & 0.00442 & 0.00023 & 0.68731 \\
\hline 3.47891 & 0.00358 & 0.00018 & 0.69578 \\
\hline 3.52188 & 0.00412 & 0.00021 & 0.70438 \\
\hline 3.565 & 0.00304 & 0.00015 & 0.713 \\
\hline 3.6075 & 0.00115 & 0.00006 & 0.7215 \\
\hline 3.64969 & 0.00072 & 0.00004 & 0.72994 \\
\hline 3.69156 & 0.00149 & 0.00008 & 0.73831 \\
\hline 3.73312 & 0.00276 & 0.00014 & 0.74663 \\
\hline 3.77484 & 0.0024 & 0.00012 & 0.75497 \\
\hline 3.81719 & 0.00299 & 0.00015 & 0.76344 \\
\hline 3.85969 & 0.00444 & 0.00023 & 0.77194 \\
\hline 3.90219 & 0.00484 & 0.00025 & 0.78044 \\
\hline 3.94469 & 0.00354 & 0.00018 & 0.78894 \\
\hline 3.98703 & 0.00238 & 0.00012 & 0.79741 \\
\hline 4.02938 & 0.0013 & 0.00007 & 0.80588 \\
\hline 4.07172 & 0.00068 & 0.00003 & 0.81434 \\
\hline 4.11453 & 0.00143 & 0.00007 & 0.82291 \\
\hline 4.15719 & 0.00334 & 0.00017 & 0.83144 \\
\hline 4.2 & 0.00577 & 0.00029 & 0.84 \\
\hline 4.24234 & 0.00521 & 0.00027 & 0.84847 \\
\hline 4.28469 & 0.00424 & 0.00022 & 0.85694 \\
\hline 4.32672 & 0.0025 & 0.00013 & 0.86534 \\
\hline 4.36828 & 0.00208 & 0.00011 & 0.87366 \\
\hline 4.41016 & 0.00138 & 0.00007 & 0.88203 \\
\hline 4.45266 & 0.00258 & 0.00013 & 0.89053 \\
\hline 4.49453 & 0.00321 & 0.00016 & 0.89891 \\
\hline 4.53641 & 0.00194 & 0.0001 & 0.90728 \\
\hline 4.57906 & 0.00233 & 0.00012 & 0.91581 \\
\hline 4.6225 & 0.0051 & 0.00026 & 0.9245 \\
\hline 4.66547 & 0.00652 & 0.00033 & 0.93309 \\
\hline 4.70797 & 0.00536 & 0.00027 & 0.94159 \\
\hline 4.75062 & 0.00402 & 0.0002 & 0.95012 \\
\hline 4.79312 & 0.00371 & 0.00019 & 0.95862 \\
\hline 4.83547 & 0.00458 & 0.00023 & 0.96709 \\
\hline 4.87781 & 0.00601 & 0.00031 & 0.97556 \\
\hline 4.92016 & 0.00566 & 0.00029 & 0.98403 \\
\hline 4.96203 & 0.00432 & 0.00022 & 0.99241 \\
\hline 5.00328 & 0.00351 & 0.00018 & 1.00066 \\
\hline 5.04516 & 0.00329 & 0.00017 & 1.00903 \\
\hline 5.08766 & 0.00449 & 0.00023 & 1.01753 \\
\hline 5.13016 & 0.00608 & 0.00031 & 1.02603 \\
\hline 5.17219 & 0.00679 & 0.00035 & 1.03444 \\
\hline 5.21453 & 0.00557 & 0.00028 & 1.04291 \\
\hline 5.25719 & 0.00306 & 0.00016 & 1.05144 \\
\hline 5.29953 & 0.0024 & 0.00012 & 1.05991 \\
\hline 5.3425 & 0.00207 & 0.00011 & 1.0685 \\
\hline 5.38609 & 0.0028 & 0.00014 & 1.07722 \\
\hline 5.42891 & 0.00388 & 0.0002 & 1.08578 \\
\hline 5.47094 & 0.00266 & 0.00014 & 1.09419 \\
\hline 5.51312 & 0.001 & 0.00005 & 1.10263 \\
\hline
\end{tabular}




\begin{tabular}{|c|c|c|c|}
\hline $\begin{array}{l}\text { Extension } \\
\text { (mm) }\end{array}$ & $\begin{array}{l}\text { Load } \\
\text { (N) }\end{array}$ & \begin{tabular}{|c|} 
Compressive stress \\
(MPa]
\end{tabular} & $\begin{array}{c}\text { Compressive strain } \\
{[\mathrm{mm} / \mathrm{mm}]}\end{array}$ \\
\hline 5.47094 & 0.00266 & 0.00014 & 1.09419 \\
\hline 5.51312 & 0.001 & 0.00005 & 1.10263 \\
\hline 5.55531 & 0.00078 & 0.00004 & 1.11106 \\
\hline 5.59656 & 0.00216 & 0.00011 & 1.11931 \\
\hline 5.63781 & 0.0044 & 0.00022 & 1.12756 \\
\hline 5.68016 & 0.00753 & 0.00038 & 1.13603 \\
\hline 5.72281 & 0.00822 & 0.00042 & 1.14456 \\
\hline 5.76484 & 0.00613 & 0.00031 & 1.15297 \\
\hline 5.80703 & 0.00379 & 0.00019 & 1.16141 \\
\hline 5.84969 & 0.00354 & 0.00018 & 1.16994 \\
\hline 5.89234 & 0.00384 & 0.0002 & 1.17847 \\
\hline 5.93453 & 0.00191 & 0.0001 & 1.18691 \\
\hline 5.97687 & 0.00133 & 0.00007 & 1.19537 \\
\hline 6.01969 & 0.00285 & 0.00014 & 1.20394 \\
\hline 6.06266 & 0.00412 & 0.00021 & 1.21253 \\
\hline 6.10547 & 0.00504 & 0.00026 & 1.22109 \\
\hline 6.14781 & 0.00642 & 0.00033 & 1.22956 \\
\hline 6.19 & 0.00642 & 0.00033 & 1.238 \\
\hline 6.23156 & 0.00269 & 0.00014 & 1.24631 \\
\hline 6.27359 & 0.00253 & 0.00013 & 1.25472 \\
\hline 6.31578 & 0.00582 & 0.0003 & 1.26316 \\
\hline 6.35797 & 0.00601 & 0.00031 & 1.27159 \\
\hline 6.4 & 0.00473 & 0.00024 & 1.28 \\
\hline 6.44266 & 0.00551 & 0.00028 & 1.28853 \\
\hline 6.48484 & 0.00512 & 0.00026 & 1.29697 \\
\hline 6.52672 & 0.00242 & 0.00012 & 1.30534 \\
\hline 6.56906 & 0.00214 & 0.00011 & 1.31381 \\
\hline 6.61203 & 0.00539 & 0.00027 & 1.32241 \\
\hline 6.655 & 0.00612 & 0.00031 & 1.331 \\
\hline 6.69766 & 0.00386 & 0.0002 & 1.33953 \\
\hline 6.74016 & 0.00317 & 0.00016 & 1.34803 \\
\hline 6.7825 & 0.00473 & 0.00024 & 1.3565 \\
\hline 6.82437 & 0.00464 & 0.00024 & 1.36487 \\
\hline 6.86578 & 0.00349 & 0.00018 & 1.37316 \\
\hline 6.90766 & 0.00517 & 0.00026 & 1.38153 \\
\hline 6.95 & 0.00632 & 0.00032 & 1.39 \\
\hline 6.99219 & 0.00517 & 0.00026 & 1.39844 \\
\hline 7.03453 & 0.00422 & 0.00022 & 1.40691 \\
\hline 7.0775 & 0.00605 & 0.00031 & 1.4155 \\
\hline 7.12047 & 0.00695 & 0.00035 & 1.42409 \\
\hline 7.16281 & 0.00408 & 0.00021 & 1.43256 \\
\hline 7.20531 & 0.00161 & 0.00008 & 1.44106 \\
\hline 7.24813 & 0.00205 & 0.0001 & 1.44963 \\
\hline 7.29094 & 0.00425 & 0.00022 & 1.45819 \\
\hline 7.33344 & 0.00565 & 0.00029 & 1.46669 \\
\hline 7.37578 & 0.00517 & 0.00026 & 1.47516 \\
\hline 7.41781 & 0.00377 & 0.00019 & 1.48356 \\
\hline 7.45984 & 0.00168 & 0.00009 & 1.49197 \\
\hline 7.50156 & 0.00111 & 0.00006 & 1.50031 \\
\hline 7.54359 & 0.00325 & 0.00017 & 1.50872 \\
\hline 7.58578 & 0.0044 & 0.00022 & 1.51716 \\
\hline 7.62797 & 0.00489 & 0.00025 & 1.52559 \\
\hline 7.67016 & 0.00696 & 0.00035 & 1.53403 \\
\hline 7.71234 & 0.00776 & 0.0004 & 1.54247 \\
\hline 7.75438 & 0.00372 & 0.00019 & 1.55088 \\
\hline 7.79688 & 0.00104 & 0.00005 & 1.55938 \\
\hline 7.83969 & 0.0037 & 0.00019 & 1.56794 \\
\hline 7.88281 & 0.00495 & 0.00025 & 1.57656 \\
\hline 7.92547 & 0.00252 & 0.00013 & 1.58509 \\
\hline 7.96781 & 0.00081 & 0.00004 & 1.59356 \\
\hline 8.01 & 0.00021 & 0.00001 & 1.602 \\
\hline 8.05203 & 0.00131 & 0.00007 & 1.61041 \\
\hline 8.09406 & 0.00406 & 0.00021 & 1.61881 \\
\hline 8.13547 & 0.00448 & 0.00023 & 1.62709 \\
\hline 8.1775 & 0.00826 & 0.00042 & 1.6355 \\
\hline
\end{tabular}

\begin{tabular}{|c|c|c|c|}
\hline $\begin{array}{c}\text { Extension } \\
\text { (mm) }\end{array}$ & $\begin{array}{c}\text { Load } \\
\text { (N) }\end{array}$ & $\begin{array}{c}\text { Compressive stress } \\
\text { (MPa] }\end{array}$ & $\begin{array}{c}\text { Compressive strain } \\
\text { [mm/mm] }\end{array}$ \\
\hline 8.26219 & 0.01072 & 0.00055 & 1.65244 \\
\hline 8.30469 & 0.00624 & 0.00032 & 1.66094 \\
\hline 8.34719 & 0.00411 & 0.00021 & 1.66944 \\
\hline 8.38953 & 0.00409 & 0.00021 & 1.67791 \\
\hline 8.43141 & 0.00272 & 0.00014 & 1.68628 \\
\hline 8.47391 & 0.00155 & 0.00008 & 1.69478 \\
\hline 8.51703 & 0.00522 & 0.00027 & 1.70341 \\
\hline 8.55953 & 0.00894 & 0.00046 & 1.71191 \\
\hline 8.60188 & 0.00745 & 0.00038 & 1.72038 \\
\hline 8.64422 & 0.00272 & 0.00014 & 1.72884 \\
\hline 8.68641 & 0.00223 & 0.00011 & 1.73728 \\
\hline 8.72813 & 0.00462 & 0.00024 & 1.74563 \\
\hline 8.77 & 0.00571 & 0.00029 & 1.754 \\
\hline 8.81234 & 0.00799 & 0.00041 & 1.76247 \\
\hline 8.85484 & 0.01047 & 0.00053 & 1.77097 \\
\hline 8.89703 & 0.00915 & 0.00047 & 1.77941 \\
\hline 8.93969 & 0.00532 & 0.00027 & 1.78794 \\
\hline 8.98281 & 0.00391 & 0.0002 & 1.79656 \\
\hline 9.02547 & 0.00568 & 0.00029 & 1.80509 \\
\hline 9.06766 & 0.00537 & 0.00027 & 1.81353 \\
\hline 9.10984 & 0.00363 & 0.00019 & 1.82197 \\
\hline 9.15234 & 0.00404 & 0.00021 & 1.83047 \\
\hline 9.19516 & 0.00534 & 0.00027 & 1.83903 \\
\hline 9.23766 & 0.00643 & 0.00033 & 1.84753 \\
\hline 9.27984 & 0.00561 & 0.00029 & 1.85597 \\
\hline 9.32172 & 0.0046 & 0.00023 & 1.86434 \\
\hline 9.36312 & 0.004 & 0.0002 & 1.87262 \\
\hline 9.405 & 0.003 & 0.00015 & 1.881 \\
\hline 9.44734 & 0.00305 & 0.00016 & 1.88947 \\
\hline 9.48969 & 0.00536 & 0.00027 & 1.89794 \\
\hline 9.53219 & 0.01022 & 0.00052 & 1.90644 \\
\hline 9.57484 & 0.01391 & 0.00071 & 1.91497 \\
\hline 9.6175 & 0.01274 & 0.00065 & 1.9235 \\
\hline 9.66 & 0.01061 & 0.00054 & 1.932 \\
\hline 9.7025 & 0.00868 & 0.00044 & 1.9405 \\
\hline 9.74547 & 0.00719 & 0.00037 & 1.94909 \\
\hline 9.78844 & 0.00669 & 0.00034 & 1.95769 \\
\hline 9.83094 & 0.00609 & 0.00031 & 1.96619 \\
\hline 9.87328 & 0.00763 & 0.00039 & 1.97466 \\
\hline 9.91531 & 0.00797 & 0.00041 & 1.98306 \\
\hline 9.95687 & 0.00867 & 0.00044 & 1.99137 \\
\hline 9.99828 & 0.019 & 0.00097 & 1.99966 \\
\hline 10.04031 & 0.05415 & 0.00276 & 2.00806 \\
\hline 10.08266 & 0.13163 & 0.0067 & 2.01653 \\
\hline 10.125 & 0.26242 & 0.01337 & 2.025 \\
\hline 10.16734 & 0.4462 & 0.02272 & 2.03347 \\
\hline 10.21 & 0.67589 & 0.03442 & 2.042 \\
\hline 10.25266 & 0.93635 & 0.04769 & 2.05053 \\
\hline 10.29547 & 1.2176 & 0.06201 & 2.05909 \\
\hline 10.33813 & 1.51494 & 0.07716 & 2.06763 \\
\hline 10.38094 & 1.82638 & 0.09302 & 2.07619 \\
\hline 10.42359 & 2.1515 & 0.10958 & 2.08472 \\
\hline 10.46594 & 2.49258 & 0.12695 & 2.09319 \\
\hline 10.50813 & 2.85391 & 0.14535 & 2.10162 \\
\hline 10.55 & 3.23808 & 0.16491 & 2.11 \\
\hline 10.59141 & 3.65499 & 0.18615 & 2.11828 \\
\hline 10.63297 & 4.12131 & 0.2099 & 2.12659 \\
\hline 10.67484 & 4.64365 & 0.2365 & 2.13497 \\
\hline 10.71734 & 5.22072 & 0.26589 & 2.14347 \\
\hline 10.75969 & 5.8479 & 0.29783 & 2.15194 \\
\hline 10.80203 & 6.51838 & 0.33198 & 2.16041 \\
\hline 10.845 & 7.22921 & 0.36818 & 2.169 \\
\hline 10.88797 & 7.96369 & 0.40559 & 2.17759 \\
\hline 10.93031 & 8.58521 & 0.43724 & 2.18606 \\
\hline 10.97297 & 9.00171 & 0.45845 & 2.19459 \\
\hline
\end{tabular}




\begin{tabular}{|c|c|c|c|}
\hline $\begin{array}{l}\text { Extension } \\
\text { (mm) }\end{array}$ & $\begin{array}{l}\text { Load } \\
\text { (N) }\end{array}$ & $\begin{array}{c}\text { Compressive stress } \\
\text { (MPa) }\end{array}$ & $\begin{array}{c}\text { Compressive strain } \\
\text { [mm/mm }\end{array}$ \\
\hline 11.05812 & 9.50424 & 0.48405 & 2.21162 \\
\hline 11.10016 & 9.79314 & 0.49876 & 2.22003 \\
\hline 11.14203 & 10.39079 & 0.5292 & 2.22841 \\
\hline 11.18406 & 11.23995 & 0.57245 & 2.23681 \\
\hline 11.22578 & 12.26098 & 0.62445 & 2.24516 \\
\hline 11.2675 & 13.40378 & 0.68265 & 2.2535 \\
\hline 11.30984 & 14.63714 & 0.74546 & 2.26197 \\
\hline 11.3525 & 15.95763 & 0.81272 & 2.2705 \\
\hline 11.39484 & 17.36797 & 0.88454 & 2.27897 \\
\hline 11.43719 & 18.8672 & 0.9609 & 2.28744 \\
\hline 11.48 & 20.46645 & 1.04235 & 2.296 \\
\hline 11.5225 & 22.17985 & 1.12961 & 2.3045 \\
\hline 11.565 & 24.00723 & 1.22268 & 2.313 \\
\hline 11.60766 & 25.96341 & 1.32231 & 2.32153 \\
\hline 11.65047 & 28.07069 & 1.42963 & 2.33009 \\
\hline 11.69281 & 30.34074 & 1.54524 & 2.33856 \\
\hline 11.73484 & 32.77691 & 1.66931 & 2.34697 \\
\hline 11.77719 & 35.39027 & 1.80241 & 2.35544 \\
\hline 11.81938 & 38.19099 & 1.94505 & 2.36388 \\
\hline 11.86063 & 41.17299 & 2.09692 & 2.37213 \\
\hline 11.90219 & 44.33416 & 2.25792 & 2.38044 \\
\hline 11.94438 & 47.7252 & 2.43062 & 2.38888 \\
\hline 11.98703 & 51.40145 & 2.61785 & 2.39741 \\
\hline 12.02953 & 55.37825 & 2.82039 & 2.40591 \\
\hline 12.07219 & 59.6725 & 3.0391 & 2.41444 \\
\hline 12.115 & 64.32105 & 3.27584 & 2.423 \\
\hline 12.1575 & 69.34299 & 3.53161 & 2.4315 \\
\hline 12.19969 & 74.73737 & 3.80634 & 2.43994 \\
\hline 12.24203 & 80.50349 & 4.10001 & 2.44841 \\
\hline 12.28453 & 86.70141 & 4.41567 & 2.45691 \\
\hline 12.32703 & 93.36797 & 4.75519 & 2.46541 \\
\hline 12.36922 & 100.48454 & 5.11764 & 2.47384 \\
\hline 12.41156 & 108.08588 & 5.50477 & 2.48231 \\
\hline 12.45328 & 116.23923 & 5.92002 & 2.49066 \\
\hline 12.49453 & 124.96115 & 6.36422 & 2.49891 \\
\hline 12.53625 & 134.24548 & 6.83707 & 2.50725 \\
\hline 12.57859 & 144.20064 & 7.34408 & 2.51572 \\
\hline 12.62063 & 154.97623 & 7.89287 & 2.52413 \\
\hline 12.6625 & 166.59021 & 8.48437 & 2.5325 \\
\hline 12.70469 & 179.05968 & 9.11943 & 2.54094 \\
\hline 12.74719 & 192.48811 & 9.80334 & 2.54944 \\
\hline 12.78953 & 207.02203 & 10.54355 & 2.55791 \\
\hline 12.83187 & 222.68954 & 11.34149 & 2.56638 \\
\hline 12.87422 & 239.5228 & 12.1988 & 2.57484 \\
\hline 12.91687 & 257.63748 & 13.12137 & 2.58337 \\
\hline 12.95891 & 277.10275 & 14.11273 & 2.59178 \\
\hline 13.00078 & 297.98544 & 15.17627 & 2.60016 \\
\hline 13.04266 & 320.35437 & 16.31551 & 2.60853 \\
\hline 13.08406 & 344.32468 & 17.53631 & 2.61681 \\
\hline 13.125 & 370.05347 & 18.84667 & 2.625 \\
\hline 13.16687 & 397.58365 & 20.24877 & 2.63337 \\
\hline 13.20875 & 427.20599 & 21.75742 & 2.64175 \\
\hline 13.24219 & 452.64185 & 23.05286 & 2.64844 \\
\hline
\end{tabular}


Table D.8 ODM-Soaked Scaffolds Without Osteoblasts Specimen 4 Raw Compression Data

\begin{tabular}{|c|c|c|c|}
\hline $\begin{array}{l}\text { Extension } \\
\text { (mm) }\end{array}$ & $\begin{array}{c}\text { Load } \\
\text { (N) }\end{array}$ & \begin{tabular}{|c|} 
Compressive stress \\
(MPa)
\end{tabular} & $\begin{array}{c}\text { Compressive strain } \\
\text { [mm/mm] }\end{array}$ \\
\hline 0 & 0.00509 & 0.00026 & 0 \\
\hline 0.00203 & 0.00493 & 0.00025 & 0.00041 \\
\hline 0.01688 & 0.01287 & 0.00066 & 0.00338 \\
\hline 0.05469 & 0.04866 & 0.00248 & 0.01094 \\
\hline 0.10344 & 0.0827 & 0.00421 & 0.02069 \\
\hline 0.15 & 0.07854 & 0.004 & 0.03 \\
\hline 0.19422 & 0.04628 & 0.00236 & 0.03884 \\
\hline 0.24156 & 0.02152 & 0.0011 & 0.04831 \\
\hline 0.29234 & 0.01486 & 0.00076 & 0.05847 \\
\hline 0.34266 & 0.01164 & 0.00059 & 0.06853 \\
\hline 0.39047 & 0.00341 & 0.00017 & 0.07809 \\
\hline 0.43703 & -0.00442 & -0.00023 & 0.08741 \\
\hline 0.48344 & -0.00639 & -0.00033 & 0.09669 \\
\hline 0.52969 & -0.00581 & -0.0003 & 0.10594 \\
\hline 0.575 & -0.00616 & -0.00031 & 0.115 \\
\hline 0.62031 & -0.00192 & -0.0001 & 0.12406 \\
\hline 0.66578 & 0.00197 & 0.0001 & 0.13316 \\
\hline 0.71078 & 0.00337 & 0.00017 & 0.14216 \\
\hline 0.75531 & 0.00233 & 0.00012 & 0.15106 \\
\hline 0.79937 & 0.00079 & 0.00004 & 0.15987 \\
\hline 0.84312 & 0.00075 & 0.00004 & 0.16862 \\
\hline 0.88703 & 0.00029 & 0.00001 & 0.17741 \\
\hline 0.93141 & -0.00062 & -0.00003 & 0.18628 \\
\hline 0.97547 & -0.00144 & -0.00007 & 0.19509 \\
\hline 1.01891 & -0.00151 & -0.00008 & 0.20378 \\
\hline 1.06172 & -0.00177 & -0.00009 & 0.21234 \\
\hline 1.10453 & -0.00078 & -0.00004 & 0.22091 \\
\hline 1.14719 & 0.00137 & 0.00007 & 0.22944 \\
\hline 1.18953 & 0.00214 & 0.00011 & 0.23791 \\
\hline 1.23219 & 0.00085 & 0.00004 & 0.24644 \\
\hline 1.275 & -0.00035 & -0.00002 & 0.255 \\
\hline 1.31766 & 0.0012 & 0.00006 & 0.26353 \\
\hline 1.36047 & 0.00162 & 0.00008 & 0.27209 \\
\hline 1.40313 & 0.00044 & 0.00002 & 0.28063 \\
\hline 1.44609 & 0.00106 & 0.00005 & 0.28922 \\
\hline 1.48875 & 0.00228 & 0.00012 & 0.29775 \\
\hline 1.53141 & 0.00144 & 0.00007 & 0.30628 \\
\hline 1.57438 & 0.00144 & 0.00007 & 0.31488 \\
\hline 1.61703 & 0.00351 & 0.00018 & 0.32341 \\
\hline 1.65953 & 0.00561 & 0.00029 & 0.33191 \\
\hline 1.70172 & 0.00449 & 0.00023 & 0.34034 \\
\hline 1.74391 & 0.00101 & 0.00005 & 0.34878 \\
\hline 1.78562 & -0.00038 & -0.00002 & 0.35712 \\
\hline 1.8275 & -0.00015 & -0.00001 & 0.3655 \\
\hline 1.86969 & 0.00166 & 0.00008 & 0.37394 \\
\hline 1.91188 & 0.00365 & 0.00019 & 0.38238 \\
\hline 1.95406 & 0.00513 & 0.00026 & 0.39081 \\
\hline 1.99703 & 0.00629 & 0.00032 & 0.39941 \\
\hline 2.04031 & 0.00577 & 0.00029 & 0.40806 \\
\hline 2.08312 & 0.00524 & 0.00027 & 0.41662 \\
\hline 2.12578 & 0.00468 & 0.00024 & 0.42516 \\
\hline 2.16859 & 0.004 & 0.0002 & 0.43372 \\
\hline 2.21125 & 0.00152 & 0.00008 & 0.44225 \\
\hline 2.25375 & -0.00094 & -0.00005 & 0.45075 \\
\hline 2.29594 & -0.0005 & -0.00003 & 0.45919 \\
\hline 2.33813 & 0.00088 & 0.00005 & 0.46763 \\
\hline 2.37984 & 0.00157 & 0.00008 & 0.47597 \\
\hline 2.42125 & 0.00137 & 0.00007 & 0.48425 \\
\hline 2.46313 & 0.00273 & 0.00014 & 0.49263 \\
\hline 2.50547 & 0.00283 & 0.00014 & 0.50109 \\
\hline 2.54781 & 0.00221 & 0.00011 & 0.50956 \\
\hline 2.59031 & 0.00403 & 0.00021 & 0.51806 \\
\hline 2.63297 & 0.00539 & 0.00027 & 0.52659 \\
\hline 2.67547 & 0.00319 & 0.00016 & 0.53509 \\
\hline 2.71766 & -0.00023 & -0.00001 & 0.54353 \\
\hline
\end{tabular}

\begin{tabular}{|c|c|c|c|}
\hline $\begin{array}{c}\text { Extension } \\
\text { (mm) }\end{array}$ & $\begin{array}{l}\text { Load } \\
\text { (N) }\end{array}$ & $\begin{array}{c}\text { Compressive stress } \\
\text { (MPa) }\end{array}$ & $\begin{array}{c}\text { Compressive strain } \\
\text { [mmimm] }\end{array}$ \\
\hline 2.80234 & 0.00127 & 0.00006 & 0.56047 \\
\hline 2.845 & 0.00208 & 0.00011 & 0.569 \\
\hline 2.88766 & 0.00165 & 0.00008 & 0.57753 \\
\hline 2.93 & 0.00317 & 0.00016 & 0.586 \\
\hline 2.97219 & 0.00392 & 0.0002 & 0.59444 \\
\hline 3.01359 & 0.00293 & 0.00015 & 0.60272 \\
\hline 3.05516 & 0.00231 & 0.00012 & 0.61103 \\
\hline 3.09766 & 0.00385 & 0.0002 & 0.61953 \\
\hline 3.14047 & 0.00625 & 0.00032 & 0.62809 \\
\hline 3.18281 & 0.00347 & 0.00018 & 0.63656 \\
\hline 3.22516 & 0.00001 & 0 & 0.64503 \\
\hline 3.26766 & 0.00119 & 0.00006 & 0.65353 \\
\hline 3.30984 & 0.00385 & 0.0002 & 0.66197 \\
\hline 3.35188 & 0.00392 & 0.0002 & 0.67038 \\
\hline 3.39422 & 0.003 & 0.00015 & 0.67884 \\
\hline 3.43703 & 0.00471 & 0.00024 & 0.68741 \\
\hline 3.48016 & 0.00434 & 0.00022 & 0.69603 \\
\hline 3.52234 & -0.00051 & -0.00003 & 0.70447 \\
\hline 3.56437 & -0.00236 & -0.00012 & 0.71288 \\
\hline 3.60672 & -0.00003 & 0 & 0.72134 \\
\hline 3.64906 & 0.00155 & 0.00008 & 0.72981 \\
\hline 3.69094 & -0.00075 & -0.00004 & 0.73819 \\
\hline 3.73281 & 0.00049 & 0.00003 & 0.74656 \\
\hline 3.77516 & 0.00451 & 0.00023 & 0.75503 \\
\hline 3.81734 & 0.00619 & 0.00032 & 0.76347 \\
\hline 3.85969 & 0.00617 & 0.00031 & 0.77194 \\
\hline 3.90203 & 0.0056 & 0.00029 & 0.78041 \\
\hline 3.94453 & 0.00331 & 0.00017 & 0.78891 \\
\hline 3.98734 & 0.00106 & 0.00005 & 0.79747 \\
\hline 4.03016 & 0.00055 & 0.00003 & 0.80603 \\
\hline 4.07281 & 0.0024 & 0.00012 & 0.81456 \\
\hline 4.11531 & 0.00187 & 0.0001 & 0.82306 \\
\hline 4.15766 & 0.00032 & 0.00002 & 0.83153 \\
\hline 4.19984 & 0.001 & 0.00005 & 0.83997 \\
\hline 4.24172 & 0.00166 & 0.00008 & 0.84834 \\
\hline 4.28266 & 0.00228 & 0.00012 & 0.85653 \\
\hline 4.32438 & 0.00214 & 0.00011 & 0.86488 \\
\hline 4.36766 & 0.00481 & 0.00024 & 0.87353 \\
\hline 4.41062 & 0.00512 & 0.00026 & 0.88212 \\
\hline 4.45281 & 0.00256 & 0.00013 & 0.89056 \\
\hline 4.49516 & 0.0003 & 0.00002 & 0.89903 \\
\hline 4.53797 & 0.00144 & 0.00007 & 0.90759 \\
\hline 4.58078 & 0.00435 & 0.00022 & 0.91616 \\
\hline 4.62313 & 0.00335 & 0.00017 & 0.92463 \\
\hline 4.66609 & 0.00254 & 0.00013 & 0.93322 \\
\hline 4.70906 & 0.00376 & 0.00019 & 0.94181 \\
\hline 4.75141 & 0.00475 & 0.00024 & 0.95028 \\
\hline 4.79359 & 0.00404 & 0.00021 & 0.95872 \\
\hline 4.83547 & 0.00351 & 0.00018 & 0.96709 \\
\hline 4.87703 & 0.00297 & 0.00015 & 0.97541 \\
\hline 4.91844 & 0.0027 & 0.00014 & 0.98369 \\
\hline 4.96031 & 0.00285 & 0.00015 & 0.99206 \\
\hline 5.00266 & 0.00511 & 0.00026 & 1.00053 \\
\hline 5.045 & 0.00747 & 0.00038 & 1.009 \\
\hline 5.08719 & 0.00677 & 0.00034 & 1.01744 \\
\hline 5.13031 & 0.00632 & 0.00032 & 1.02606 \\
\hline 5.17359 & 0.00631 & 0.00032 & 1.03472 \\
\hline 5.21594 & 0.00618 & 0.00031 & 1.04319 \\
\hline 5.25813 & 0.00389 & 0.0002 & 1.05163 \\
\hline 5.30109 & 0.00249 & 0.00013 & 1.06022 \\
\hline 5.34406 & 0.00446 & 0.00023 & 1.06881 \\
\hline 5.38656 & 0.00357 & 0.00018 & 1.07731 \\
\hline 5.42875 & 0.00162 & 0.00008 & 1.08575 \\
\hline 5.47094 & 0.00075 & 0.00004 & 1.09419 \\
\hline 5.5125 & 0.00112 & 0.00006 & 1.1025 \\
\hline
\end{tabular}




\begin{tabular}{|c|c|c|c|}
\hline $\begin{array}{c}\text { Extension } \\
(\mathrm{mm})\end{array}$ & $\begin{array}{l}\text { Load } \\
\text { (N) }\end{array}$ & $\begin{array}{c}\text { Compressive stress } \\
\text { (MPa) }\end{array}$ & $\begin{array}{c}\text { Compressive strain } \\
\text { (mmimm) }\end{array}$ \\
\hline 5.21594 & 0.00618 & 0.00031 & 1.04319 \\
\hline 5.25813 & 0.00389 & 0.0002 & 1.05163 \\
\hline 5.30109 & 0.00249 & 0.00013 & 1.06022 \\
\hline 5.34406 & 0.00446 & 0.00023 & 1.06881 \\
\hline 5.38656 & 0.00357 & 0.00018 & 1.07731 \\
\hline 5.42875 & 0.00162 & 0.00008 & 1.08575 \\
\hline 5.47094 & 0.00075 & 0.00004 & 1.09419 \\
\hline 5.5125 & 0.00112 & 0.00006 & 1.1025 \\
\hline 5.55375 & 0.00231 & 0.00012 & 1.11075 \\
\hline 5.59562 & 0.00367 & 0.00019 & 1.11913 \\
\hline 5.63797 & 0.00538 & 0.00027 & 1.12759 \\
\hline 5.68 & 0.00593 & 0.0003 & 1.136 \\
\hline 5.72203 & 0.00618 & 0.00031 & 1.14441 \\
\hline 5.76453 & 0.00626 & 0.00032 & 1.15291 \\
\hline 5.80734 & 0.00568 & 0.00029 & 1.16147 \\
\hline 5.85031 & 0.00531 & 0.00027 & 1.17006 \\
\hline 5.89328 & 0.00539 & 0.00027 & 1.17866 \\
\hline 5.93594 & 0.00285 & 0.00015 & 1.18719 \\
\hline 5.97844 & 0.00125 & 0.00006 & 1.19569 \\
\hline 6.02078 & 0.00215 & 0.00011 & 1.20416 \\
\hline 6.06297 & 0.00163 & 0.00008 & 1.21259 \\
\hline 6.105 & -0.00089 & -0.00005 & 1.221 \\
\hline 6.14687 & -0.00085 & -0.00004 & 1.22938 \\
\hline 6.18875 & 0.00168 & 0.00009 & 1.23775 \\
\hline 6.23125 & 0.00288 & 0.00015 & 1.24625 \\
\hline 6.27359 & 0.00465 & 0.00024 & 1.25472 \\
\hline 6.31578 & 0.00633 & 0.00032 & 1.26316 \\
\hline 6.35828 & 0.00669 & 0.00034 & 1.27166 \\
\hline 6.40047 & 0.00466 & 0.00024 & 1.28009 \\
\hline 6.44219 & 0.0018 & 0.00009 & 1.28844 \\
\hline 6.48469 & 0.00258 & 0.00013 & 1.29694 \\
\hline 6.52766 & 0.0042 & 0.00021 & 1.30553 \\
\hline 6.57047 & 0.00376 & 0.00019 & 1.31409 \\
\hline 6.61297 & 0.00116 & 0.00006 & 1.32259 \\
\hline 6.65547 & 0.00041 & 0.00002 & 1.33109 \\
\hline 6.69766 & 0.00186 & 0.00009 & 1.33953 \\
\hline 6.73969 & 0.00268 & 0.00014 & 1.34794 \\
\hline 6.78109 & 0.00244 & 0.00012 & 1.35622 \\
\hline 6.82297 & 0.0022 & 0.00011 & 1.36459 \\
\hline 6.86531 & 0.00322 & 0.00016 & 1.37306 \\
\hline 6.90766 & 0.00473 & 0.00024 & 1.38153 \\
\hline 6.94984 & 0.00562 & 0.00029 & 1.38997 \\
\hline 6.9925 & 0.0062 & 0.00032 & 1.3985 \\
\hline 7.03516 & 0.00672 & 0.00034 & 1.40703 \\
\hline 7.07813 & 0.00535 & 0.00027 & 1.41563 \\
\hline 7.12094 & 0.00382 & 0.00019 & 1.42419 \\
\hline 7.16391 & 0.00478 & 0.00024 & 1.43278 \\
\hline 7.20656 & 0.00515 & 0.00026 & 1.44131 \\
\hline 7.24875 & 0.00393 & 0.0002 & 1.44975 \\
\hline 7.29109 & 0.00436 & 0.00022 & 1.45822 \\
\hline 7.33328 & 0.00516 & 0.00026 & 1.46666 \\
\hline 7.37516 & 0.00278 & 0.00014 & 1.47503 \\
\hline 7.41688 & 0.00219 & 0.00011 & 1.48338 \\
\hline 7.45891 & 0.00468 & 0.00024 & 1.49178 \\
\hline 7.50125 & 0.00655 & 0.00033 & 1.50025 \\
\hline 7.54344 & 0.00545 & 0.00028 & 1.50869 \\
\hline 7.58578 & 0.004 & 0.0002 & 1.51716 \\
\hline 7.62828 & 0.00398 & 0.0002 & 1.52566 \\
\hline 7.67047 & 0.00461 & 0.00023 & 1.53409 \\
\hline 7.7125 & 0.00354 & 0.00018 & 1.5425 \\
\hline 7.75453 & 0.0037 & 0.00019 & 1.55091 \\
\hline 7.79766 & 0.00616 & 0.00031 & 1.55953 \\
\hline 7.84063 & 0.0078 & 0.0004 & 1.56813 \\
\hline 7.88312 & 0.00567 & 0.00029 & 1.57662 \\
\hline 7.92531 & 0.00468 & 0.00024 & 1.58506 \\
\hline
\end{tabular}

\begin{tabular}{|c|c|c|c|}
\hline $\begin{array}{c}\text { Extension } \\
\text { (mm) }\end{array}$ & $\begin{array}{l}\text { Load } \\
\text { (N) }\end{array}$ & $\begin{array}{c}\text { Compressive stress } \\
\text { (MPa) }\end{array}$ & $\begin{array}{c}\text { Compressive strain } \\
{[\mathrm{mm} / \mathrm{mm}]}\end{array}$ \\
\hline 8.00922 & 0.00562 & \begin{tabular}{|c|}
0.00029 \\
\end{tabular} & 1.60184 \\
\hline 8.05078 & 0.0031 & 0.00016 & 1.61016 \\
\hline 8.09297 & 0.00201 & 0.0001 & 1.61859 \\
\hline 8.13547 & 0.00359 & 0.00018 & 1.62709 \\
\hline 8.17781 & 0.00573 & 0.00029 & 1.63556 \\
\hline 8.22 & 0.00547 & 0.00028 & 1.644 \\
\hline 8.26234 & 0.00503 & 0.00026 & 1.65247 \\
\hline 8.30516 & 0.00485 & 0.00025 & 1.66103 \\
\hline 8.34766 & 0.00346 & 0.00018 & 1.66953 \\
\hline 8.39016 & 0.00215 & 0.00011 & 1.67803 \\
\hline 8.43266 & 0.00295 & 0.00015 & 1.68653 \\
\hline 8.47516 & 0.00557 & 0.00028 & 1.69503 \\
\hline 8.5175 & 0.00481 & 0.00025 & 1.7035 \\
\hline 8.55984 & 0.00156 & 0.00008 & 1.71197 \\
\hline 8.60203 & 0.00087 & 0.00004 & 1.72041 \\
\hline 8.64375 & 0.00132 & 0.00007 & 1.72875 \\
\hline 8.68547 & 0.00187 & 0.0001 & 1.73709 \\
\hline 8.72766 & 0.00449 & 0.00023 & 1.74553 \\
\hline 8.77 & 0.00801 & 0.00041 & 1.754 \\
\hline 8.81234 & 0.00797 & 0.00041 & 1.76247 \\
\hline 8.855 & 0.00667 & 0.00034 & 1.771 \\
\hline 8.89797 & 0.00682 & 0.00035 & 1.77959 \\
\hline 8.94062 & 0.00654 & 0.00033 & 1.78812 \\
\hline 8.9825 & 0.0044 & 0.00022 & 1.7965 \\
\hline 9.025 & 0.00307 & 0.00016 & 1.805 \\
\hline 9.06812 & 0.00409 & 0.00021 & 1.81362 \\
\hline 9.11094 & 0.00584 & 0.0003 & 1.82219 \\
\hline 9.15313 & 0.00607 & 0.00031 & 1.83063 \\
\hline 9.19547 & 0.00449 & 0.00023 & 1.83909 \\
\hline 9.23734 & 0.00414 & 0.00021 & 1.84747 \\
\hline 9.27859 & 0.00283 & 0.00014 & 1.85572 \\
\hline 9.32031 & 0.00257 & 0.00013 & 1.86406 \\
\hline 9.36281 & 0.00423 & 0.00022 & 1.87256 \\
\hline 9.40516 & 0.006 & 0.00031 & 1.88103 \\
\hline 9.44734 & 0.00735 & 0.00037 & 1.88947 \\
\hline 9.49 & 0.00647 & 0.00033 & 1.898 \\
\hline 9.53281 & 0.005 & 0.00025 & 1.90656 \\
\hline 9.57547 & 0.00567 & 0.00029 & 1.91509 \\
\hline 9.61797 & 0.00922 & 0.00047 & 1.92359 \\
\hline 9.66078 & 0.0259 & 0.00132 & 1.93216 \\
\hline 9.70344 & 0.10694 & 0.00545 & 1.94069 \\
\hline 9.74594 & 0.29876 & 0.01522 & 1.94919 \\
\hline 9.78812 & 0.64058 & 0.03262 & 1.95762 \\
\hline 9.83031 & 1.17598 & 0.05989 & 1.96606 \\
\hline 9.87203 & 1.90205 & 0.09687 & 1.97441 \\
\hline 9.91359 & 2.76591 & 0.14087 & 1.98272 \\
\hline 9.95562 & 3.70844 & 0.18887 & 1.99112 \\
\hline 9.99813 & 4.68916 & 0.23882 & 1.99963 \\
\hline 10.04063 & 5.69307 & 0.28995 & 2.00813 \\
\hline 10.08297 & 6.70776 & 0.34162 & 2.01659 \\
\hline 10.12547 & 7.66432 & 0.39034 & 2.02509 \\
\hline 10.16812 & 8.35139 & 0.42533 & 2.03362 \\
\hline 10.21078 & 8.81542 & 0.44897 & 2.04216 \\
\hline 10.25328 & 9.15023 & 0.46602 & 2.05066 \\
\hline 10.29609 & 9.48988 & 0.48332 & 2.05922 \\
\hline 10.33875 & 10.07662 & 0.5132 & 2.06775 \\
\hline 10.38125 & 10.93207 & 0.55677 & 2.07625 \\
\hline 10.42344 & 11.98147 & 0.61021 & 2.08469 \\
\hline 10.46516 & 13.16049 & 0.67026 & 2.09303 \\
\hline 10.50672 & 14.42087 & 0.73445 & 2.10134 \\
\hline 10.54828 & 15.73634 & 0.80145 & 2.10966 \\
\hline 10.59031 & 17.09441 & 0.87061 & 2.11806 \\
\hline 10.63266 & 18.49092 & 0.94174 & 2.12653 \\
\hline 10.67516 & 19.93348 & 1.0152 & 2.13503 \\
\hline 10.71766 & 21.43184 & 1.09151 & 2.14353 \\
\hline
\end{tabular}




\begin{tabular}{|c|c|c|c|}
\hline $\begin{array}{l}\text { Extension } \\
\text { [mm] }\end{array}$ & $\begin{array}{l}\text { Load } \\
\text { (N) }\end{array}$ & \begin{tabular}{|c|} 
Compressive stress \\
(MPa)
\end{tabular} & $\begin{array}{c}\text { Compressive strain } \\
\text { [mm?mm] }\end{array}$ \\
\hline 10.80328 & 24.61543 & \begin{tabular}{|c|}
1.25365 \\
\end{tabular} & 2.16066 \\
\hline 10.84562 & 26.31601 & 1.34026 & 2.16913 \\
\hline 10.88797 & 28.07954 & 1.43008 & 2.17759 \\
\hline 10.93062 & 29.91876 & 1.52375 & 2.18612 \\
\hline 10.97312 & 31.85239 & 1.62223 & 2.19462 \\
\hline 11.015 & 33.87836 & 1.72541 & 2.203 \\
\hline 11.05719 & 36.00122 & 1.83353 & 2.21144 \\
\hline 11.09922 & 38.22251 & 1.94666 & 2.21984 \\
\hline 11.14094 & 40.55753 & 2.06558 & 2.22819 \\
\hline 11.18266 & 43.01923 & 2.19095 & 2.23653 \\
\hline 11.22484 & 45.60577 & 2.32268 & 2.24497 \\
\hline 11.26719 & 48.34149 & 2.46201 & 2.25344 \\
\hline 11.30953 & 51.2351 & 2.60938 & 2.26191 \\
\hline 11.35203 & 54.27941 & 2.76443 & 2.27041 \\
\hline 11.39484 & 57.47527 & 2.92719 & 2.27897 \\
\hline 11.43766 & 60.84005 & 3.09856 & 2.28753 \\
\hline 11.48 & 64.36849 & 3.27826 & 2.296 \\
\hline 11.5225 & 68.05787 & 3.46616 & 2.3045 \\
\hline 11.56516 & 71.92583 & 3.66315 & 2.31303 \\
\hline 11.60766 & 75.98539 & 3.8699 & 2.32153 \\
\hline 11.65 & 80.238 & 4.08649 & 2.33 \\
\hline 11.69203 & 84.70663 & 4.31407 & 2.33841 \\
\hline 11.73391 & 89.40456 & 4.55334 & 2.34678 \\
\hline 11.77547 & 94.34798 & 4.8051 & 2.35509 \\
\hline 11.81719 & 99.54963 & 5.07002 & 2.36344 \\
\hline 11.85953 & 105.06197 & 5.35076 & 2.37191 \\
\hline 11.90203 & 110.96237 & 5.65127 & 2.38041 \\
\hline 11.94406 & 117.2498 & 5.97148 & 2.38881 \\
\hline 11.98641 & 123.91078 & 6.31072 & 2.39728 \\
\hline 12.02891 & 130.94736 & 6.66909 & 2.40578 \\
\hline 12.07156 & 138.41605 & 7.04947 & 2.41431 \\
\hline 12.11406 & 146.35551 & 7.45382 & 2.42281 \\
\hline 12.15672 & 154.75343 & 7.88153 & 2.43134 \\
\hline 12.19969 & 163.6582 & 8.33504 & 2.43994 \\
\hline 12.24203 & 173.13687 & 8.81779 & 2.44841 \\
\hline 12.28406 & 183.13515 & 9.327 & 2.45681 \\
\hline 12.32609 & 193.61557 & 9.86076 & 2.46522 \\
\hline 12.36781 & 204.68135 & 10.42434 & 2.47356 \\
\hline 12.40922 & 216.4594 & 11.02419 & 2.48184 \\
\hline 12.45062 & 228.99815 & 11.66278 & 2.49012 \\
\hline 12.49266 & 242.43277 & 12.347 & 2.49853 \\
\hline 12.53484 & 256.95441 & 13.08658 & 2.50697 \\
\hline 12.57687 & 272.63055 & 13.88496 & 2.51537 \\
\hline 12.61922 & 289.40936 & 14.7395 & 2.52384 \\
\hline 12.66141 & 307.37344 & 15.6544 & 2.53228 \\
\hline 12.70375 & 326.72504 & 16.63997 & 2.54075 \\
\hline 12.74594 & 347.64227 & 17.70527 & 2.54919 \\
\hline 12.78813 & 370.29791 & 18.85912 & 2.55763 \\
\hline 12.83031 & 394.89081 & 20.11162 & 2.56606 \\
\hline 12.8725 & 421.58963 & 21.47138 & 2.5745 \\
\hline 12.91453 & 450.6297 & 22.95038 & 2.58291 \\
\hline
\end{tabular}


Table D.9 PBS-Soaked Scaffolds Specimen 1 Raw Compression Data

\begin{tabular}{|c|c|c|c|c|c|c|c|}
\hline $\begin{array}{c}\text { Extension } \\
\text { (mm) }\end{array}$ & $\begin{array}{l}\text { Load } \\
\text { (N) }\end{array}$ & $\begin{array}{c}\text { Compressive stress } \\
\text { (MPa] }\end{array}$ & $\begin{array}{c}\text { Compressive strain } \\
\text { [mm/mm] }\end{array}$ & $\begin{array}{c}\text { Extension } \\
\text { (mm) }\end{array}$ & $\begin{array}{c}\text { Load } \\
\text { (N) }\end{array}$ & $\begin{array}{c}\text { Compressive stress } \\
\text { [MPa] }\end{array}$ & $\begin{array}{c}\text { Compressive strain } \\
{[\mathrm{mm} / \mathrm{mm}]}\end{array}$ \\
\hline 0 & 0.00124 & 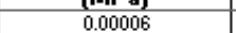 & (Iі) & 2.80344 & -0.00193 & -0.0001 & 0.56069 \\
\hline 0.00203 & 0.00286 & 0.00015 & 0.00041 & 2.84578 & -0.00302 & -0.00015 & 0.56916 \\
\hline 0.01797 & 0.01538 & 0.00078 & 0.00359 & 2.88813 & -0.00458 & -0.00023 & 0.57763 \\
\hline 0.05672 & 0.04745 & 0.00242 & 0.01134 & 2.93047 & -0.00389 & -0.0002 & 0.58609 \\
\hline 0.10359 & 0.07656 & 0.0039 & 0.02072 & 2.97266 & -0.00083 & -0.00004 & 0.59453 \\
\hline 0.14703 & 0.06937 & 0.00353 & 0.02941 & 3.01484 & -0.00009 & 0 & 0.60297 \\
\hline 0.19016 & 0.04088 & 0.00208 & 0.03803 & 3.05687 & -0.00048 & -0.00002 & 0.61137 \\
\hline 0.23844 & 0.02388 & 0.00122 & 0.04769 & 3.09828 & -0.00048 & -0.00002 & 0.61966 \\
\hline 0.28969 & 0.01985 & 0.00101 & 0.05794 & 3.14 & -0.00074 & -0.00004 & 0.628 \\
\hline 0.33969 & 0.01355 & 0.00069 & 0.06794 & 3.1825 & 0.00078 & 0.00004 & 0.6365 \\
\hline 0.38781 & 0.00051 & 0.00003 & 0.07756 & 3.22531 & 0.00474 & 0.00024 & 0.64506 \\
\hline 0.43531 & -0.00769 & -0.00039 & 0.08706 & 3.26734 & 0.00562 & 0.00029 & 0.65347 \\
\hline 0.48266 & -0.00559 & -0.00028 & 0.09653 & 3.30922 & 0.00339 & 0.00017 & 0.66184 \\
\hline 0.52953 & -0.00363 & -0.00018 & 0.10591 & 3.35172 & 0.00235 & 0.00012 & 0.67034 \\
\hline 0.57516 & -0.00585 & -0.0003 & 0.11503 & 3.39422 & 0.00264 & 0.00013 & 0.67884 \\
\hline 0.61984 & .0 .00742 & -0.00038 & 0.12397 & 3.43672 & 0.00349 & 0.00018 & 0.68734 \\
\hline 0.66469 & -0.00505 & -0.00026 & 0.13294 & 3.47938 & 0.00247 & 0.00013 & 0.69588 \\
\hline 0.71016 & -0.00146 & -0.00007 & 0.14203 & 3.52234 & 0.00062 & 0.00003 & 0.70447 \\
\hline 0.755 & -0.00074 & -0.00004 & 0.151 & 3.565 & -0.0012 & -0.00006 & 0.713 \\
\hline 0.79906 & -0.00107 & -0.00005 & 0.15981 & 3.60766 & -0.00258 & -0.00013 & 0.72153 \\
\hline 0.84312 & -0.00142 & -0.00007 & 0.16862 & 3.65 & -0.00223 & -0.00011 & 0.73 \\
\hline 0.88688 & -0.00129 & -0.00007 & 0.17738 & 3.69187 & -0.00227 & -0.00012 & 0.73838 \\
\hline 0.93047 & -0.00122 & .0 .00006 & 0.18609 & 3.73344 & -0.00177 & -0.00009 & 0.74669 \\
\hline 0.97422 & -0.00045 & -0.00002 & 0.19484 & 3.77547 & 0.0003 & 0.00002 & 0.75509 \\
\hline 1.01812 & 0.00043 & 0.00002 & 0.20362 & 3.81766 & 0.0019 & 0.0001 & 0.76353 \\
\hline 1.06172 & -0.00102 & -0.00005 & 0.21234 & 3.85984 & 0.00322 & 0.00016 & 0.77197 \\
\hline 1.105 & -0.00368 & -0.00019 & 0.221 & 3.90234 & 0.00478 & 0.00024 & 0.78047 \\
\hline 1.14797 & -0.00445 & -0.00023 & 0.22959 & 3.94516 & 0.00486 & 0.00025 & 0.78903 \\
\hline 1.19047 & -0.00356 & -0.00018 & 0.23809 & 3.98766 & 0.00112 & 0.00006 & 0.79753 \\
\hline 1.2325 & -0.00215 & -0.00011 & 0.2465 & 4.02984 & -0.0043 & -0.00022 & 0.80597 \\
\hline 1.27469 & -0.00075 & -0.00004 & 0.25494 & 4.07141 & -0.00653 & -0.00033 & 0.81428 \\
\hline 1.3175 & 0.00145 & 0.00007 & 0.2635 & 4.11375 & -0.00474 & -0.00024 & 0.82275 \\
\hline 1.36016 & 0.0019 & 0.0001 & 0.27203 & 4.15734 & -0.0024 & -0.00012 & 0.83147 \\
\hline 1.4025 & 0.00101 & 0.00005 & 0.2805 & 4.20063 & -0.00003 & 0 & 0.84013 \\
\hline 1.44531 & 0.00068 & 0.00003 & 0.28906 & 4.24281 & 0.00083 & 0.00004 & 0.84856 \\
\hline 1.48828 & 0.00159 & 0.00008 & 0.29766 & 4.28453 & -0.00065 & -0.00003 & 0.85691 \\
\hline 1.53125 & 0.00166 & 0.00008 & 0.30625 & 4.32641 & -0.00346 & -0.00018 & 0.86528 \\
\hline 1.57406 & -0.00126 & -0.00006 & 0.31481 & 4.36812 & -0.00136 & -0.00007 & 0.87362 \\
\hline 1.61672 & -0.00432 & -0.00022 & 0.32334 & 4.41031 & 0.0035 & 0.00018 & 0.88206 \\
\hline 1.65953 & -0.00343 & -0.00017 & 0.33191 & 4.45297 & 0.00645 & 0.00033 & 0.89059 \\
\hline 1.70234 & -0.00007 & 0 & 0.34047 & 4.49531 & 0.0068 & 0.00035 & 0.89906 \\
\hline 1.74484 & 0.00048 & 0.00002 & 0.34897 & 4.53766 & 0.005 & 0.00025 & 0.90753 \\
\hline 1.78719 & -0.00024 & -0.00001 & 0.35744 & 4.58031 & 0.004 & 0.0002 & 0.91606 \\
\hline 1.82891 & $\begin{array}{l}-0.000065 \\
-0.00065\end{array}$ & -0.00003 & 0.36578 & 4.62313 & 0.00295 & 0.00015 & 0.92463 \\
\hline 1.87047 & -0.00019 & -0.00001 & 0.37409 & 4.66563 & 0.00013 & 0.00001 & 0.93313 \\
\hline 1.91266 & 0.00061 & 0.00003 & 0.38253 & 4.70797 & -0.0018 & -0.00009 & 0.94159 \\
\hline 1.95516 & 0.00431 & 0.00022 & 0.39103 & 4.75031 & -0.0018 & -0.00009 & 0.95006 \\
\hline 1.99734 & 0.00688 & 0.00035 & 0.39947 & 4.79266 & -0.00202 & -0.0001 & 0.95853 \\
\hline 2.03984 & 0.00431 & 0.00022 & 0.40797 & 4.83484 & $\begin{array}{l}-0.00166 \\
\end{array}$ & -0.00008 & 0.96697 \\
\hline 2.08266 & 0.00068 & 0.00003 & 0.41653 & 4.87797 & 0.0006 & 0.00003 & 0.97559 \\
\hline 2.12547 & -0.00037 & -0.00002 & 0.42509 & 4.92109 & 0.0047 & 0.00024 & 0.98422 \\
\hline 2.16781 & 0.00006 & 0 & 0.43356 & 4.96281 & 0.00474 & 0.00024 & 0.99256 \\
\hline 2.21047 & 0.00001 & 0 & 0.44209 & 5.00391 & 0.00103 & 0.00005 & 1.00078 \\
\hline 2.25344 & -0.00006 & 0 & 0.45069 & 5.04609 & 0.00015 & 0.00001 & 1.00922 \\
\hline 2.29609 & 0.00001 & 0 & 0.45922 & 5.08875 & 0.00157 & 0.00008 & 1.01775 \\
\hline 2.33844 & -0.00196 & -0.0001 & 0.46769 & 5.13109 & 0.0016 & 0.00008 & 1.02622 \\
\hline 2.38063 & -0.00353 & -0.00018 & 0.47613 & 5.17313 & 0.0008 & 0.00004 & 1.03463 \\
\hline 2.4225 & -0.0029 & -0.00015 & 0.4845 & 5.21547 & 0.00115 & 0.00006 & 1.04309 \\
\hline 2.46406 & -0.00157 & -0.00008 & 0.49281 & 5.25813 & 0.00142 & 0.00007 & 1.05163 \\
\hline 2.50531 & -0.00111 & -0.00006 & 0.50106 & 5.30063 & -0.00043 & -0.00002 & 1.06013 \\
\hline 2.54688 & -0.0017 & -0.00009 & 0.50937 & 5.34328 & -0.00181 & -0.00009 & 1.06866 \\
\hline 2.58922 & 0.00016 & 0.00001 & 0.51784 & 5.38609 & -0.00134 & -0.00007 & 1.07722 \\
\hline 2.63188 & 0.00323 & 0.00016 & 0.52637 & 5.42875 & -0.00145 & -0.00007 & 1.08575 \\
\hline 2.675 & 0.00516 & 0.00026 & 0.535 & 5.47078 & -0.00374 & -0.00019 & 1.09416 \\
\hline 2.71813 & 0.00379 & 0.00019 & 0.54362 & 5.5125 & -0.00419 & -0.00021 & 1.1025 \\
\hline
\end{tabular}




\begin{tabular}{|c|c|c|c|}
\hline $\begin{array}{l}\text { Extension } \\
\text { (mm) }\end{array}$ & $\begin{array}{c}\text { Load } \\
\text { (N) }\end{array}$ & $\begin{array}{c}\text { Compressive stress } \\
\text { (MPa) }\end{array}$ & $\begin{array}{c}\text { Compressive strain } \\
(\mathrm{mm} / \mathrm{mm})\end{array}$ \\
\hline 5.59656 & 0.00244 & 0.00012 & 1.11931 \\
\hline 5.63875 & 0.00364 & 0.00019 & 1.12775 \\
\hline 5.68094 & 0.00485 & 0.00025 & 1.13619 \\
\hline 5.72297 & 0.00578 & 0.00029 & 1.14459 \\
\hline 5.76531 & 0.00464 & 0.00024 & 1.15306 \\
\hline 5.80781 & 0.00387 & 0.0002 & 1.16156 \\
\hline 5.85047 & 0.00647 & 0.00033 & 1.17009 \\
\hline 5.89313 & 0.00657 & 0.00033 & 1.17862 \\
\hline 5.93547 & 0.00324 & 0.00016 & 1.18709 \\
\hline 5.97813 & -0.0003 & -0.00002 & 1.19563 \\
\hline 6.02078 & -0.00342 & -0.00017 & 1.20416 \\
\hline 6.06328 & -0.00334 & -0.00017 & 1.21266 \\
\hline 6.10547 & -0.00184 & -0.00009 & 1.22109 \\
\hline 6.14781 & -0.00033 & -0.00002 & 1.22956 \\
\hline 6.18969 & 0.00178 & 0.00009 & 1.23794 \\
\hline 6.23109 & 0.0025 & 0.00013 & 1.24622 \\
\hline 6.27297 & 0.00164 & 0.00008 & 1.25459 \\
\hline 6.31531 & 0.00212 & 0.00011 & 1.26306 \\
\hline 6.35797 & 0.00477 & 0.00024 & 1.27159 \\
\hline 6.40047 & 0.00528 & 0.00027 & 1.28009 \\
\hline 6.44266 & 0.00074 & 0.00004 & 1.28853 \\
\hline 6.48484 & -0.00266 & -0.00014 & 1.29697 \\
\hline 6.52703 & -0.00195 & -0.0001 & 1.30541 \\
\hline 6.56953 & -0.00216 & -0.00011 & 1.31391 \\
\hline 6.61266 & -0.00288 & -0.00015 & 1.32253 \\
\hline 6.65562 & -0.00141 & -0.00007 & 1.33113 \\
\hline 6.69828 & 0.00095 & 0.00005 & 1.33966 \\
\hline 6.74062 & 0.00185 & 0.00009 & 1.34812 \\
\hline 6.78281 & 0.00261 & 0.00013 & 1.35656 \\
\hline 6.82453 & 0.00346 & 0.00018 & 1.36491 \\
\hline 6.86609 & 0.00377 & 0.00019 & 1.37322 \\
\hline 6.90828 & 0.0032 & 0.00016 & 1.38166 \\
\hline 6.95063 & 0.00084 & 0.00004 & 1.39013 \\
\hline 6.99281 & -0.00072 & -0.00004 & 1.39856 \\
\hline 7.03516 & 0.00093 & 0.00005 & 1.40703 \\
\hline 7.07797 & 0.00283 & 0.00014 & 1.41559 \\
\hline 7.12078 & 0.00378 & 0.00019 & 1.42416 \\
\hline 7.16344 & 0.0028 & 0.00014 & 1.43269 \\
\hline 7.20578 & 0.00189 & 0.0001 & 1.44116 \\
\hline 7.24844 & 0.00258 & 0.00013 & 1.44969 \\
\hline 7.29141 & 0.00316 & 0.00016 & 1.45828 \\
\hline 7.33391 & 0.00415 & 0.00021 & 1.46678 \\
\hline 7.37594 & 0.00427 & 0.00022 & 1.47519 \\
\hline 7.41813 & 0.00179 & 0.00009 & 1.48363 \\
\hline 7.46031 & -0.0009 & -0.00005 & 1.49206 \\
\hline 7.50219 & -0.00052 & -0.00003 & 1.50044 \\
\hline 7.54422 & 0.00258 & 0.00013 & 1.50884 \\
\hline 7.58641 & 0.00407 & 0.00021 & 1.51728 \\
\hline 7.62875 & 0.00479 & 0.00024 & 1.52575 \\
\hline 7.67109 & 0.00509 & 0.00026 & 1.53422 \\
\hline 7.71328 & 0.00511 & 0.00026 & 1.54266 \\
\hline 7.755 & 0.00276 & 0.00014 & 1.551 \\
\hline 7.79703 & 0.00265 & 0.00014 & 1.55941 \\
\hline 7.83969 & 0.00328 & 0.00017 & 1.56794 \\
\hline 7.88266 & 0.00104 & 0.00005 & 1.57653 \\
\hline 7.92547 & -0.00211 & -0.00011 & 1.58509 \\
\hline 7.96766 & -0.00339 & -0.00017 & 1.59353 \\
\hline 8.00984 & -0.00233 & -0.00012 & 1.60197 \\
\hline 8.05219 & -0.00006 & 0 & 1.61044 \\
\hline 8.09406 & 0.00251 & 0.00013 & 1.61881 \\
\hline 8.13578 & 0.0027 & 0.00014 & 1.62716 \\
\hline 8.17797 & 0.00184 & 0.00009 & 1.63559 \\
\hline 8.22047 & 0.00202 & 0.0001 & 1.64409 \\
\hline 8.26297 & 0.00357 & 0.00018 & 1.65259 \\
\hline 8.30531 & 0.0044 & 0.00022 & 1.66106 \\
\hline
\end{tabular}

\begin{tabular}{|c|c|c|c|}
\hline $\begin{array}{c}\text { Extension } \\
\text { (mm) }\end{array}$ & $\begin{array}{c}\text { Load } \\
\text { (N) }\end{array}$ & $\begin{array}{c}\text { Compressive stress } \\
\text { (MPa] }\end{array}$ & $\begin{array}{c}\text { Compressive strain } \\
{[\mathrm{mm} / \mathrm{mm}]}\end{array}$ \\
\hline 8.39031 & 0.00295 & 0.00015 & 1.67806 \\
\hline 8.4325 & 0.00019 & 0.00001 & 1.6865 \\
\hline 8.47469 & -0.00087 & -0.00004 & 1.69494 \\
\hline 8.51734 & 0.0001 & 0.00001 & 1.70347 \\
\hline 8.56 & 0.00055 & 0.00003 & 1.712 \\
\hline 8.60234 & 0.00141 & 0.00007 & 1.72047 \\
\hline 8.64469 & 0.00293 & 0.00015 & 1.72894 \\
\hline 8.68703 & 0.0038 & 0.00019 & 1.73741 \\
\hline 8.72875 & 0.00188 & 0.0001 & 1.74575 \\
\hline 8.77047 & 0.00122 & 0.00006 & 1.75409 \\
\hline 8.8125 & 0.0027 & 0.00014 & 1.7625 \\
\hline 8.85469 & 0.00188 & 0.0001 & 1.77094 \\
\hline 8.89703 & -0.00104 & -0.00005 & 1.77941 \\
\hline 8.93969 & 0.00088 & 0.00004 & 1.78794 \\
\hline 8.9825 & 0.00406 & 0.00021 & 1.7965 \\
\hline 9.02547 & 0.00508 & 0.00026 & 1.80509 \\
\hline 9.06797 & 0.00521 & 0.00027 & 1.81359 \\
\hline 9.11031 & 0.00472 & 0.00024 & 1.82206 \\
\hline 9.15297 & 0.00337 & 0.00017 & 1.83059 \\
\hline 9.19563 & 0.00175 & 0.00009 & 1.83913 \\
\hline 9.23781 & 0.00262 & 0.00013 & 1.84756 \\
\hline 9.28 & 0.0028 & 0.00014 & 1.856 \\
\hline 9.32187 & 0.00164 & 0.00008 & 1.86437 \\
\hline 9.36359 & -0.00013 & -0.00001 & 1.87272 \\
\hline 9.40531 & $\begin{array}{c}-0.00166 \\
\end{array}$ & -0.00008 & 1.88106 \\
\hline 9.44766 & -0.00027 & -0.00001 & 1.88953 \\
\hline 9.49016 & 0.0013 & 0.00007 & 1.89803 \\
\hline 9.53266 & 0.00224 & 0.00011 & 1.90653 \\
\hline 9.57516 & 0.00223 & 0.00011 & 1.91503 \\
\hline 9.61766 & 0.00257 & 0.00013 & 1.92353 \\
\hline 9.66031 & 0.0043 & 0.00022 & 1.93206 \\
\hline 9.70281 & 0.00532 & 0.00027 & 1.94056 \\
\hline 9.74562 & 0.00421 & 0.00021 & 1.94912 \\
\hline 9.78844 & 0.00307 & 0.00016 & 1.95769 \\
\hline 9.83078 & 0.00022 & 0.00001 & 1.96616 \\
\hline 9.87313 & 0.0069 & 0.00035 & 1.97462 \\
\hline 9.91531 & 0.06151 & 0.00313 & 1.98306 \\
\hline 9.95687 & 0.20004 & 0.01019 & 1.99137 \\
\hline 9.99844 & 0.43486 & 0.02215 & 1.99969 \\
\hline 10.04047 & 0.75437 & 0.03842 & 2.00809 \\
\hline 10.08313 & 1.13162 & 0.05763 & 2.01662 \\
\hline 10.12547 & 1.54092 & 0.07848 & 2.02509 \\
\hline 10.16781 & 1.97498 & 0.10058 & 2.03356 \\
\hline 10.21031 & 2.44134 & 0.12434 & 2.04206 \\
\hline 10.25313 & 2.9425 & 0.14986 & 2.05063 \\
\hline 10.29578 & 3.47763 & 0.17711 & 2.05916 \\
\hline 10.33875 & 4.04624 & 0.20607 & 2.06775 \\
\hline 10.38156 & 4.6485 & 0.23675 & 2.07631 \\
\hline 10.42406 & 5.28177 & 0.269 & 2.08481 \\
\hline 10.46656 & 5.94081 & 0.30256 & 2.09331 \\
\hline 10.50859 & 6.62775 & 0.33755 & 2.10172 \\
\hline 10.55031 & 7.31143 & 0.37237 & 2.11006 \\
\hline 10.59172 & 7.82633 & 0.39859 & 2.11834 \\
\hline 10.63313 & 8.12038 & 0.41357 & 2.12663 \\
\hline 10.67516 & 8.30377 & 0.42291 & 2.13503 \\
\hline 10.71766 & 8.48842 & 0.43231 & 2.14353 \\
\hline 10.75984 & 8.88573 & 0.45255 & 2.15197 \\
\hline 10.8025 & 9.54839 & 0.4863 & 2.1605 \\
\hline 10.84531 & 10.40316 & 0.52983 & 2.16906 \\
\hline 10.88812 & 11.3884 & 0.58001 & 2.17762 \\
\hline 10.93031 & 12.46352 & 0.63476 & 2.18606 \\
\hline 10.97266 & 13.59826 & 0.69255 & 2.19453 \\
\hline 11.01531 & 14.78558 & 0.75302 & 2.20306 \\
\hline 11.05797 & 16.03511 & 0.81666 & 2.21159 \\
\hline 11.10016 & 17.34682 & 0.88347 & 2.22003 \\
\hline
\end{tabular}




\begin{tabular}{|c|c|c|c|}
\hline $\begin{array}{l}\text { Extension } \\
\text { (mm) }\end{array}$ & $\begin{array}{l}\text { Load } \\
\text { (N) }\end{array}$ & $\begin{array}{c}\text { Compressive stress } \\
\text { (MPa] }\end{array}$ & $\begin{array}{c}\text { Compressive strain } \\
\text { [mm/mm] }\end{array}$ \\
\hline 11.18453 & 20.18003 & 1.02776 & 2.23691 \\
\hline 11.22625 & 21.70566 & 1.10546 & 2.24525 \\
\hline 11.26828 & 23.30662 & 1.187 & 2.25366 \\
\hline 11.31047 & 24.99747 & 1.27311 & 2.26209 \\
\hline 11.35281 & 26.81148 & 1.3655 & 2.27056 \\
\hline 11.39484 & 28.76196 & 1.46483 & 2.27897 \\
\hline 11.43719 & 30.85801 & 1.57159 & 2.28744 \\
\hline 11.48016 & 33.12499 & 1.68704 & 2.29603 \\
\hline 11.52266 & 35.57507 & 1.81182 & 2.30453 \\
\hline 11.56516 & 38.20217 & 1.94562 & 2.31303 \\
\hline 11.60766 & 41.01351 & 2.0888 & 2.32153 \\
\hline 11.65031 & 44.02914 & 2.24239 & 2.33006 \\
\hline 11.69297 & 47.25464 & 2.40666 & 2.33859 \\
\hline 11.73516 & 50.67723 & 2.58097 & 2.34703 \\
\hline 11.77734 & 54.30725 & 2.76585 & 2.35547 \\
\hline 11.81922 & 58.15248 & 2.96168 & 2.36384 \\
\hline 11.86063 & 62.22141 & 3.16891 & 2.37213 \\
\hline 11.9025 & 66.52386 & 3.38803 & 2.3805 \\
\hline 11.94484 & 71.10442 & 3.62132 & 2.38897 \\
\hline 11.98734 & 76.00471 & 3.87089 & 2.39747 \\
\hline 12.02938 & 81.23363 & 4.1372 & 2.40587 \\
\hline 12.07203 & 86.79249 & 4.42031 & 2.41441 \\
\hline 12.11484 & 92.71573 & 4.72197 & 2.42297 \\
\hline 12.15719 & 99.06086 & 5.04513 & 2.43144 \\
\hline 12.19938 & 105.83073 & 5.38991 & 2.43988 \\
\hline 12.24219 & 113.02185 & 5.75616 & 2.44844 \\
\hline 12.285 & 120.72215 & 6.14833 & 2.457 \\
\hline 12.32719 & 128.90057 & 6.56485 & 2.46544 \\
\hline 12.36906 & 137.50032 & 7.00283 & 2.47381 \\
\hline 12.41141 & 146.53177 & 7.4628 & 2.48228 \\
\hline 12.45297 & 156.09889 & 7.95005 & 2.49059 \\
\hline 12.49422 & 166.25346 & 8.46722 & 2.49884 \\
\hline 12.53594 & 177.05774 & 9.01748 & 2.50719 \\
\hline 12.57812 & 188.70059 & 9.61044 & 2.51562 \\
\hline 12.62 & 201.32216 & 10.25325 & 2.524 \\
\hline 12.66187 & 214.89072 & 10.94429 & 2.53237 \\
\hline 12.70422 & 229.39235 & 11.68286 & 2.54084 \\
\hline 12.74703 & 244.91983 & 12.47366 & 2.54941 \\
\hline 12.78953 & 261.62329 & 13.32436 & 2.55791 \\
\hline 12.83172 & 279.53513 & 14.23661 & 2.56634 \\
\hline 12.87391 & 298.65005 & 15.21012 & 2.57478 \\
\hline 12.91641 & 319.08209 & 16.25072 & 2.58328 \\
\hline 12.95875 & 341.0051 & 17.36725 & 2.59175 \\
\hline 13.00063 & 364.55652 & 18.56671 & 2.60013 \\
\hline 13.04219 & 389.68082 & 19.84628 & 2.60844 \\
\hline 13.08328 & 416.33151 & 21.20359 & 2.61666 \\
\hline 13.12437 & 444.68369 & 22.64755 & 2.62487 \\
\hline 13.1325 & 450.57425 & 22.94756 & 2.6265 \\
\hline
\end{tabular}


Table D.10 PBS-Soaked Scaffolds Specimen 2 Raw Compression Data

\begin{tabular}{|c|c|c|c|c|c|c|c|}
\hline $\begin{array}{l}\text { Extension } \\
\text { (mm) }\end{array}$ & $\begin{array}{l}\text { Load } \\
\text { (N) }\end{array}$ & $\begin{array}{c}\text { Compressive stress } \\
\text { [MPa] }\end{array}$ & $\begin{array}{c}\text { Compressive strain } \\
\text { [mm/mm }]\end{array}$ & $\begin{array}{l}\text { Extension } \\
\text { (mm) }\end{array}$ & $\begin{array}{l}\text { Load } \\
\text { (N) }\end{array}$ & $\begin{array}{c}\text { Compressive stress } \\
\text { [MPa] }\end{array}$ & $\begin{array}{c}\text { Compressive strain } \\
\text { [mmimm }\end{array}$ \\
\hline 0 & -0.00017 & $\begin{array}{r}-0.00001 \\
\end{array}$ & \begin{tabular}{|c|}
0 \\
\end{tabular} & 2.80281 & 0.00384 & \begin{tabular}{|c|}
0.0002 \\
\end{tabular} & $\begin{array}{l}0.56056 \\
\end{array}$ \\
\hline 0.0025 & 0.00172 & 0.00009 & 0.0005 & 2.84516 & 0.0036 & 0.00018 & 0.56903 \\
\hline 0.02203 & 0.01813 & 0.00092 & 0.00441 & 2.88766 & 0.00275 & 0.00014 & 0.57753 \\
\hline 0.06312 & 0.05532 & 0.00282 & 0.01262 & 2.93016 & 0.00112 & 0.00006 & 0.58603 \\
\hline 0.10969 & 0.07785 & 0.00396 & 0.02194 & 2.9725 & 0.00072 & 0.00004 & 0.5945 \\
\hline 0.15188 & 0.06252 & 0.00318 & 0.03038 & 3.01453 & 0.00113 & 0.00006 & 0.60291 \\
\hline 0.19469 & 0.03619 & 0.00184 & 0.03894 & 3.05625 & 0.00014 & 0.00001 & 0.61125 \\
\hline 0.24313 & 0.02466 & 0.00126 & 0.04862 & 3.0975 & -0.0009 & -0.00005 & 0.6195 \\
\hline 0.29406 & 0.02331 & 0.00119 & 0.05881 & 3.13937 & 0.00233 & 0.00012 & 0.62787 \\
\hline 0.34297 & 0.01716 & 0.00087 & 0.06859 & 3.18219 & 0.00751 & 0.00038 & 0.63644 \\
\hline 0.39031 & 0.00528 & 0.00027 & 0.07806 & 3.22484 & 0.00913 & 0.00046 & 0.64497 \\
\hline 0.4375 & -0.00314 & -0.00016 & 0.0875 & 3.2675 & 0.00977 & 0.0005 & 0.6535 \\
\hline 0.48469 & -0.00336 & -0.00017 & 0.09694 & 3.31016 & 0.00951 & 0.00048 & 0.66203 \\
\hline 0.53141 & -0.00081 & -0.00004 & 0.10628 & 3.3525 & 0.00698 & 0.00036 & 0.6705 \\
\hline 0.57672 & -0.00111 & -0.00006 & 0.11534 & 3.39438 & 0.00264 & 0.00013 & 0.67888 \\
\hline 0.62109 & -0.00367 & -0.00019 & 0.12422 & 3.43625 & 0.00114 & 0.00006 & 0.68725 \\
\hline 0.66594 & -0.0027 & -0.00014 & 0.13319 & 3.47906 & 0.00538 & 0.00027 & 0.69581 \\
\hline 0.71109 & 0.00055 & 0.00003 & 0.14222 & 3.52234 & 0.00739 & 0.00038 & 0.70447 \\
\hline 0.75578 & 0.00276 & 0.00014 & 0.15116 & 3.565 & 0.00506 & 0.00026 & 0.713 \\
\hline 0.79984 & 0.00189 & 0.0001 & 0.15997 & 3.60734 & 0.00124 & 0.00006 & 0.72147 \\
\hline 0.84359 & 0.00371 & 0.00019 & 0.16872 & 3.64969 & 0.00105 & 0.00005 & 0.72994 \\
\hline 0.88734 & 0.00498 & 0.00025 & 0.17747 & 3.69172 & 0.00352 & 0.00018 & 0.73834 \\
\hline 0.93094 & 0.00356 & 0.00018 & 0.18619 & 3.73344 & 0.00379 & 0.00019 & 0.74669 \\
\hline 0.97453 & 0.00374 & 0.00019 & 0.19491 & 3.77516 & 0.00413 & 0.00021 & 0.75503 \\
\hline 1.01844 & 0.00396 & 0.0002 & 0.20369 & 3.81734 & 0.00677 & 0.00034 & 0.76347 \\
\hline 1.06219 & 0.00407 & 0.00021 & 0.21244 & 3.85984 & 0.00614 & 0.00031 & 0.77197 \\
\hline 1.10563 & 0.00196 & 0.0001 & 0.22113 & 3.90156 & 0.00188 & 0.0001 & 0.78031 \\
\hline 1.14844 & -0.00231 & -0.00012 & 0.22969 & 3.94359 & 0.00102 & 0.00005 & 0.78872 \\
\hline 1.19078 & -0.00343 & -0.00017 & 0.23816 & 3.98656 & 0.00526 & 0.00027 & 0.79731 \\
\hline 1.23281 & -0.00148 & -0.00008 & 0.24656 & 4.02984 & 0.00751 & 0.00038 & 0.80597 \\
\hline 1.275 & 0.00067 & 0.00003 & 0.255 & 4.0725 & 0.0064 & 0.00033 & 0.8145 \\
\hline 1.31766 & 0.00299 & 0.00015 & 0.26353 & 4.11469 & 0.00719 & 0.00037 & 0.82294 \\
\hline 1.36031 & 0.00396 & 0.0002 & 0.27206 & 4.15688 & 0.00851 & 0.00043 & 0.83138 \\
\hline 1.40234 & 0.006 & 0.00031 & 0.28047 & 4.19953 & 0.00777 & 0.0004 & 0.83991 \\
\hline 1.44469 & 0.00516 & 0.00026 & 0.28894 & 4.24219 & 0.00826 & 0.00042 & 0.84844 \\
\hline 1.48797 & 0.0047 & 0.00024 & 0.29759 & 4.28469 & 0.00807 & 0.00041 & 0.85694 \\
\hline 1.53094 & 0.00705 & 0.00036 & 0.30619 & 4.32656 & 0.00485 & 0.00025 & 0.86531 \\
\hline 1.57375 & 0.00604 & 0.00031 & 0.31475 & 4.36812 & 0.00179 & 0.00009 & 0.87362 \\
\hline 1.61641 & 0.00315 & 0.00016 & 0.32328 & 4.41031 & 0.00383 & 0.00019 & 0.88206 \\
\hline 1.65953 & 0.00233 & 0.00012 & 0.33191 & 4.45297 & 0.00831 & 0.00042 & 0.89059 \\
\hline 1.70219 & 0.00352 & 0.00018 & 0.34044 & 4.49516 & 0.00931 & 0.00047 & 0.89903 \\
\hline 1.74484 & 0.00421 & 0.00021 & 0.34897 & 4.53719 & 0.00825 & 0.00042 & 0.90744 \\
\hline 1.78719 & 0.00285 & 0.00015 & 0.35744 & 4.57922 & 0.00586 & 0.0003 & 0.91584 \\
\hline 1.82906 & 0.00308 & 0.00016 & 0.36581 & 4.62156 & 0.00595 & 0.0003 & 0.92431 \\
\hline 1.87047 & 0.00398 & 0.0002 & 0.37409 & 4.66422 & 0.0073 & 0.00037 & 0.93284 \\
\hline 1.91266 & 0.0048 & 0.00024 & 0.38253 & 4.7075 & 0.00818 & 0.00042 & 0.9415 \\
\hline 1.95516 & 0.00531 & 0.00027 & 0.39103 & 4.75141 & 0.0085 & 0.00043 & 0.95028 \\
\hline 1.9975 & 0.00715 & 0.00036 & 0.3995 & 4.79437 & 0.00617 & 0.00031 & 0.95888 \\
\hline 2.03969 & 0.00719 & 0.00037 & 0.40794 & 4.83641 & 0.00303 & 0.00015 & 0.96728 \\
\hline 2.08219 & 0.00465 & 0.00024 & 0.41644 & 4.87859 & 0.0007 & 0.00004 & 0.97572 \\
\hline 2.125 & 0.00258 & 0.00013 & 0.425 & 4.92094 & 0.00108 & 0.00005 & 0.98419 \\
\hline 2.16766 & 0.00214 & 0.00011 & 0.43353 & 4.9625 & 0.00229 & 0.00012 & 0.9925 \\
\hline 2.21016 & 0.00265 & 0.00013 & 0.44203 & 5.00375 & 0.00122 & 0.00006 & 1.00075 \\
\hline 2.25281 & 0.00396 & 0.0002 & 0.45056 & 5.04547 & 0.00124 & 0.00006 & 1.00909 \\
\hline 2.29547 & 0.00527 & 0.00027 & 0.45909 & 5.08797 & 0.00449 & 0.00023 & 1.01759 \\
\hline 2.33797 & 0.0032 & 0.00016 & 0.46759 & 5.13047 & 0.00847 & 0.00043 & 1.02609 \\
\hline 2.38031 & 0.00178 & 0.00009 & 0.47606 & 5.17266 & 0.00907 & 0.00046 & 1.03453 \\
\hline 2.42266 & 0.00152 & 0.00008 & 0.48453 & 5.21531 & 0.00722 & 0.00037 & 1.04306 \\
\hline 2.46438 & 0.00074 & 0.00004 & 0.49288 & 5.25781 & 0.00646 & 0.00033 & 1.05156 \\
\hline 2.50594 & 0.00105 & 0.00005 & 0.50119 & 5.29984 & 0.00383 & 0.00019 & 1.05997 \\
\hline 2.54781 & 0.00272 & 0.00014 & 0.50956 & 5.34188 & 0.00132 & 0.00007 & 1.06837 \\
\hline 2.59016 & 0.00315 & 0.00016 & 0.51803 & 5.38469 & 0.00318 & 0.00016 & 1.07694 \\
\hline 2.6325 & 0.00209 & 0.00011 & 0.5265 & 5.42828 & 0.00753 & 0.00038 & 1.08566 \\
\hline 2.675 & 0.00286 & 0.00015 & 0.535 & 5.47156 & 0.00595 & 0.0003 & 1.09431 \\
\hline 2.71734 & 0.00384 & 0.0002 & 0.54347 & 5.51422 & 0.00224 & 0.00011 & 1.10284 \\
\hline
\end{tabular}




\begin{tabular}{|c|c|c|c|}
\hline $\begin{array}{l}\text { Extension } \\
\text { (mm) }\end{array}$ & $\begin{array}{c}\text { Load } \\
\text { (N) }\end{array}$ & \begin{tabular}{|c|} 
Compressive stress \\
(MPa)
\end{tabular} & $\begin{array}{c}\text { Compressive strain } \\
\text { [mm/mm] }\end{array}$ \\
\hline 5.59688 & 0.0001 & 0.00001 & 1.11937 \\
\hline 5.63844 & 0.00158 & 0.00008 & 1.12769 \\
\hline 5.68062 & 0.00537 & 0.00027 & 1.13612 \\
\hline 5.72313 & 0.00867 & 0.00044 & 1.14463 \\
\hline 5.765 & 0.0077 & 0.00039 & 1.153 \\
\hline 5.80734 & 0.00411 & 0.00021 & 1.16147 \\
\hline 5.85 & 0.00291 & 0.00015 & 1.17 \\
\hline 5.89266 & 0.00437 & 0.00022 & 1.17853 \\
\hline 5.93516 & 0.00414 & 0.00021 & 1.18703 \\
\hline 5.97766 & 0.00368 & 0.00019 & 1.19553 \\
\hline 6.02016 & 0.0034 & 0.00017 & 1.20403 \\
\hline 6.0625 & 0.00444 & 0.00023 & 1.2125 \\
\hline 6.10484 & 0.00658 & 0.00034 & 1.22097 \\
\hline 6.14719 & 0.00523 & 0.00027 & 1.22944 \\
\hline 6.18953 & 0.0046 & 0.00023 & 1.23791 \\
\hline 6.23156 & 0.00517 & 0.00026 & 1.24631 \\
\hline 6.27344 & 0.00438 & 0.00022 & 1.25469 \\
\hline 6.31563 & 0.00391 & 0.0002 & 1.26313 \\
\hline 6.35813 & 0.00666 & 0.00034 & 1.27163 \\
\hline 6.40094 & 0.01183 & 0.0006 & 1.28019 \\
\hline 6.44344 & 0.01229 & 0.00063 & 1.28869 \\
\hline 6.48516 & 0.00782 & 0.0004 & 1.29703 \\
\hline 6.52719 & 0.00535 & 0.00027 & 1.30544 \\
\hline 6.56969 & 0.00579 & 0.00029 & 1.31394 \\
\hline 6.61266 & 0.00549 & 0.00028 & 1.32253 \\
\hline 6.65531 & 0.00595 & 0.0003 & 1.33106 \\
\hline 6.69766 & 0.00666 & 0.00034 & 1.33953 \\
\hline 6.74016 & 0.00587 & 0.0003 & 1.34803 \\
\hline 6.78266 & 0.00432 & 0.00022 & 1.35653 \\
\hline 6.82453 & 0.00287 & 0.00015 & 1.36491 \\
\hline 6.86594 & 0.0017 & 0.00009 & 1.37319 \\
\hline 6.90781 & 0.00361 & 0.00018 & 1.38156 \\
\hline 6.95031 & 0.00549 & 0.00028 & 1.39006 \\
\hline 6.9925 & 0.00598 & 0.0003 & 1.3985 \\
\hline 7.03484 & 0.00625 & 0.00032 & 1.40697 \\
\hline 7.0775 & 0.00677 & 0.00034 & 1.4155 \\
\hline 7.12047 & 0.00703 & 0.00036 & 1.42409 \\
\hline 7.16344 & 0.00675 & 0.00034 & 1.43269 \\
\hline 7.20594 & 0.00474 & 0.00024 & 1.44119 \\
\hline 7.24844 & 0.00381 & 0.00019 & 1.44969 \\
\hline 7.29109 & 0.0034 & 0.00017 & 1.45822 \\
\hline 7.33344 & 0.00415 & 0.00021 & 1.46669 \\
\hline 7.37594 & 0.00493 & 0.00025 & 1.47519 \\
\hline 7.41828 & 0.00638 & 0.00032 & 1.48366 \\
\hline 7.46 & 0.0045 & 0.00023 & 1.492 \\
\hline 7.50172 & 0.00177 & 0.00009 & 1.50034 \\
\hline 7.54391 & 0.00378 & 0.00019 & 1.50878 \\
\hline 7.58609 & 0.00578 & 0.00029 & 1.51722 \\
\hline 7.62828 & 0.00571 & 0.00029 & 1.52566 \\
\hline 7.67047 & 0.0062 & 0.00032 & 1.53409 \\
\hline 7.7125 & 0.00563 & 0.00029 & 1.5425 \\
\hline 7.75453 & 0.00265 & 0.00014 & 1.55091 \\
\hline 7.79656 & 0.00179 & 0.00009 & 1.55931 \\
\hline 7.83938 & 0.00452 & 0.00023 & 1.56788 \\
\hline 7.8825 & 0.00704 & 0.00036 & 1.5765 \\
\hline 7.92531 & 0.0057 & 0.00029 & 1.58506 \\
\hline 7.96797 & 0.00462 & 0.00024 & 1.59359 \\
\hline 8.01047 & 0.00511 & 0.00026 & 1.60209 \\
\hline 8.0525 & 0.00369 & 0.00019 & 1.6105 \\
\hline 8.09391 & 0.00347 & 0.00018 & 1.61878 \\
\hline 8.13547 & 0.00479 & 0.00024 & 1.62709 \\
\hline 8.17781 & 0.00671 & 0.00034 & 1.63556 \\
\hline 8.22016 & 0.00648 & 0.00033 & 1.64403 \\
\hline 8.2625 & 0.00585 & 0.0003 & 1.6525 \\
\hline 8.30484 & 0.00684 & 0.00035 & 1.66097 \\
\hline
\end{tabular}

\begin{tabular}{|c|c|c|c|}
\hline $\begin{array}{l}\text { Extension } \\
\text { [mm] }\end{array}$ & $\begin{array}{c}\text { Load } \\
\text { (N) }\end{array}$ & $\begin{array}{c}\text { Compressive stress } \\
\text { (MPa) }\end{array}$ & $\begin{array}{c}\text { Compressive strain } \\
{[\mathrm{mm} / \mathrm{mm}]}\end{array}$ \\
\hline 8.39 & 0.00911 & 0.00046 & 1.678 \\
\hline 8.43234 & 0.00589 & 0.0003 & 1.68647 \\
\hline 8.47484 & 0.00516 & 0.00026 & 1.69497 \\
\hline 8.51734 & 0.00689 & 0.00035 & 1.70347 \\
\hline 8.55984 & 0.00647 & 0.00033 & 1.71197 \\
\hline 8.60219 & 0.00576 & 0.00029 & 1.72044 \\
\hline 8.64453 & 0.00556 & 0.00028 & 1.72891 \\
\hline 8.68688 & 0.00522 & 0.00027 & 1.73738 \\
\hline 8.72859 & 0.00223 & 0.00011 & 1.74572 \\
\hline 8.77016 & 0.00069 & 0.00004 & 1.75403 \\
\hline 8.81219 & 0.00504 & 0.00026 & 1.76244 \\
\hline 8.85453 & 0.00818 & 0.00042 & 1.77091 \\
\hline 8.89719 & 0.00813 & 0.00041 & 1.77944 \\
\hline 8.94 & 0.0079 & 0.0004 & 1.788 \\
\hline 8.98297 & 0.00871 & 0.00044 & 1.79659 \\
\hline 9.02531 & 0.00873 & 0.00044 & 1.80506 \\
\hline 9.06766 & 0.00799 & 0.00041 & 1.81353 \\
\hline 9.11016 & 0.00765 & 0.00039 & 1.82203 \\
\hline 9.15266 & 0.0069 & 0.00035 & 1.83053 \\
\hline 9.19531 & 0.0037 & 0.00019 & 1.83906 \\
\hline 9.23766 & 0.00221 & 0.00011 & 1.84753 \\
\hline 9.27984 & 0.00335 & 0.00017 & 1.85597 \\
\hline 9.32156 & 0.00523 & 0.00027 & 1.86431 \\
\hline 9.36312 & 0.00389 & 0.0002 & 1.87262 \\
\hline 9.405 & 0.00302 & 0.00015 & 1.881 \\
\hline 9.44734 & 0.00514 & 0.00026 & 1.88947 \\
\hline 9.48984 & 0.00765 & 0.00039 & 1.89797 \\
\hline 9.53234 & 0.00875 & 0.00045 & 1.90647 \\
\hline 9.575 & 0.00974 & 0.0005 & 1.915 \\
\hline 9.61797 & 0.00914 & 0.00047 & 1.92359 \\
\hline 9.66047 & 0.00711 & 0.00036 & 1.93209 \\
\hline 9.70281 & 0.00484 & 0.00025 & 1.94056 \\
\hline 9.74562 & 0.00484 & 0.00025 & 1.94912 \\
\hline 9.78844 & 0.006 & 0.00031 & 1.95769 \\
\hline 9.83094 & 0.0084 & 0.00043 & 1.96619 \\
\hline 9.87297 & 0.00828 & 0.00042 & 1.97459 \\
\hline 9.915 & 0.00668 & 0.00034 & 1.983 \\
\hline 9.95672 & 0.00656 & 0.00033 & 1.99134 \\
\hline 9.99828 & 0.00652 & 0.00033 & 1.99966 \\
\hline 10.04031 & 0.0072 & 0.00037 & 2.00806 \\
\hline 10.08281 & 0.00814 & 0.00041 & 2.01656 \\
\hline 10.12531 & 0.00807 & 0.00041 & 2.02506 \\
\hline 10.16766 & 0.0086 & 0.00044 & 2.03353 \\
\hline 10.21047 & 0.03506 & 0.00179 & 2.04209 \\
\hline 10.25328 & 0.12553 & 0.00639 & 2.05066 \\
\hline 10.29578 & 0.30272 & 0.01542 & 2.05916 \\
\hline 10.33844 & 0.55426 & 0.02823 & 2.06769 \\
\hline 10.38109 & 0.86657 & 0.04413 & 2.07622 \\
\hline 10.42375 & 1.22703 & 0.06249 & 2.08475 \\
\hline 10.46625 & 1.62133 & 0.08257 & 2.09325 \\
\hline 10.50828 & 2.03889 & 0.10384 & 2.10166 \\
\hline 10.55 & 2.47227 & 0.12591 & 2.11 \\
\hline 10.59156 & 2.91954 & 0.14869 & 2.11831 \\
\hline 10.63313 & 3.37791 & 0.17204 & 2.12663 \\
\hline 10.67531 & 3.85 & 0.19608 & 2.13506 \\
\hline 10.71766 & 4.34317 & 0.2212 & 2.14353 \\
\hline 10.76 & 4.86628 & 0.24784 & 2.152 \\
\hline 10.80234 & 5.42256 & 0.27617 & 2.16047 \\
\hline 10.84516 & 6.01163 & 0.30617 & 2.16903 \\
\hline 10.88797 & 6.63939 & 0.33814 & 2.17759 \\
\hline 10.93031 & 7.30274 & 0.37193 & 2.18606 \\
\hline 10.97281 & 7.89454 & 0.40207 & 2.19456 \\
\hline 11.01562 & 8.31589 & 0.42352 & 2.20312 \\
\hline 11.05828 & 8.59655 & 0.43782 & 2.21166 \\
\hline 11.10031 & 8.80987 & 0.44868 & 2.22006 \\
\hline
\end{tabular}




\begin{tabular}{|c|c|c|c|}
\hline $\begin{array}{l}\text { Extension } \\
\text { (mm) }\end{array}$ & $\begin{array}{l}\text { Load } \\
\text { [N] }\end{array}$ & $\begin{array}{c}\text { Compressive stress } \\
\text { [MPa] }\end{array}$ & $\begin{array}{c}\text { Compressive strain } \\
{[\mathrm{mmimm}]}\end{array}$ \\
\hline 11.18422 & 9.64634 & 0.49128 & 2.23684 \\
\hline 11.22594 & 10.42852 & 0.53112 & 2.24519 \\
\hline 11.26797 & 11.36268 & 0.5787 & 2.25359 \\
\hline 11.31016 & 12.39387 & 0.63121 & 2.26203 \\
\hline 11.3525 & 13.50276 & 0.68769 & 2.2705 \\
\hline 11.39484 & 14.68513 & 0.74791 & 2.27897 \\
\hline 11.4375 & 15.95236 & 0.81245 & 2.2875 \\
\hline 11.48031 & 17.31718 & 0.88196 & 2.29606 \\
\hline 11.52281 & 18.78122 & 0.95652 & 2.30456 \\
\hline 11.56531 & 20.34599 & 1.03621 & 2.31306 \\
\hline 11.60797 & 22.02321 & 1.12163 & 2.32159 \\
\hline 11.65047 & 23.82559 & 1.21343 & 2.33009 \\
\hline 11.69297 & 25.74479 & 1.31117 & 2.33859 \\
\hline 11.73531 & 27.78571 & 1.41511 & 2.34706 \\
\hline 11.77766 & 29.96643 & 1.52618 & 2.35553 \\
\hline 11.81953 & 32.29924 & 1.64499 & 2.36391 \\
\hline 11.86078 & 34.79654 & 1.77217 & 2.37216 \\
\hline 11.90234 & 37.4753 & 1.9086 & 2.38047 \\
\hline 11.94469 & 40.38305 & 2.05669 & 2.38894 \\
\hline 11.98719 & 43.56932 & 2.21897 & 2.39744 \\
\hline 12.02969 & 47.05027 & 2.39625 & 2.40594 \\
\hline 12.07234 & 50.83776 & 2.58915 & 2.41447 \\
\hline 12.11516 & 54.9413 & 2.79814 & 2.42303 \\
\hline 12.1575 & 59.38493 & 3.02445 & 2.4315 \\
\hline 12.19984 & 64.17563 & 3.26844 & 2.43997 \\
\hline 12.2425 & 69.30537 & 3.52969 & 2.4485 \\
\hline 12.28531 & 74.81943 & 3.81052 & 2.45706 \\
\hline 12.3275 & 80.75051 & 4.11259 & 2.4655 \\
\hline 12.36953 & 87.11837 & 4.4369 & 2.47391 \\
\hline 12.41156 & 93.96339 & 4.78552 & 2.48231 \\
\hline 12.45312 & 101.35036 & 5.16173 & 2.49062 \\
\hline 12.49469 & 109.33307 & 5.56829 & 2.49894 \\
\hline 12.53672 & 117.95625 & 6.00746 & 2.50734 \\
\hline 12.57891 & 127.3131 & 6.484 & 2.51578 \\
\hline 12.62109 & 137.50554 & 7.0031 & 2.52422 \\
\hline 12.66313 & 148.53419 & 7.56478 & 2.53262 \\
\hline 12.70531 & 160.42575 & 8.17042 & 2.54106 \\
\hline 12.74781 & 173.2811 & 8.82513 & 2.54956 \\
\hline 12.79016 & 187.22183 & 9.53513 & 2.55803 \\
\hline 12.8325 & 202.29781 & 10.30294 & 2.5665 \\
\hline 12.87469 & 218.53775 & 11.13004 & 2.57494 \\
\hline 12.91719 & 236.07692 & 12.0233 & 2.58344 \\
\hline 12.95906 & 255.02562 & 12.98835 & 2.59181 \\
\hline 13.00094 & 275.44711 & 14.02841 & 2.60019 \\
\hline 13.04281 & 297.35861 & 15.14435 & 2.60856 \\
\hline 13.08406 & 320.78253 & 16.33732 & 2.61681 \\
\hline 13.12531 & 345.83563 & 17.61326 & 2.62506 \\
\hline 13.16703 & 372.57278 & 18.97498 & 2.63341 \\
\hline 13.20922 & 401.28772 & 20.43742 & 2.64184 \\
\hline 13.25078 & 432.28949 & 22.01632 & 2.65016 \\
\hline 13.2675 & 445.36853 & 22.68243 & 2.6535 \\
\hline
\end{tabular}


Table D.11 PBS-Soaked Scaffolds Specimen 3 Raw Compression Data

\begin{tabular}{|c|c|c|c|c|c|c|c|}
\hline $\begin{array}{c}\text { Extension } \\
\text { (mm) }\end{array}$ & $\begin{array}{l}\text { Load } \\
\text { (N) }\end{array}$ & $\begin{array}{c}\text { Compressive stress } \\
\text { (MPa) }\end{array}$ & $\begin{array}{c}\text { Compressive strain } \\
{[\mathrm{mm} / \mathrm{mm})}\end{array}$ & $\begin{array}{c}\text { Extension } \\
\text { (mm) }\end{array}$ & $\begin{array}{c}\text { Load } \\
\text { (N) }\end{array}$ & $\begin{array}{c}\text { Compressive stress } \\
\text { [MPa] }\end{array}$ & $\begin{array}{c}\text { Compressive strain } \\
{[\mathrm{mm} / \mathrm{mm}]}\end{array}$ \\
\hline 0 & 0.00351 & 0.00018 & $\begin{array}{c}0 \\
0 \\
\end{array}$ & 2.80234 & 0.00295 & \begin{tabular}{|c|}
0.00015 \\
\end{tabular} & 2.80234 \\
\hline 0.0025 & 0.00397 & 0.0002 & 0.0025 & 2.84484 & 0.00406 & 0.00021 & 2.84484 \\
\hline 0.02141 & 0.01867 & 0.00095 & 0.02141 & 2.88734 & 0.0049 & 0.00025 & 2.88734 \\
\hline 0.06219 & 0.05706 & 0.00291 & 0.06219 & 2.93 & 0.00057 & 0.00003 & 2.93 \\
\hline 0.10859 & 0.08097 & 0.00412 & 0.10859 & 2.9725 & -0.00093 & -0.00005 & 2.9725 \\
\hline 0.15094 & 0.06482 & 0.0033 & 0.15094 & 3.01484 & 0.00215 & 0.00011 & 3.01484 \\
\hline 0.19391 & 0.03686 & 0.00188 & 0.19391 & 3.05687 & 0.00275 & 0.00014 & 3.05687 \\
\hline 0.24234 & 0.0227 & 0.00116 & 0.24234 & 3.09844 & 0.00069 & 0.00003 & 3.09844 \\
\hline 0.29328 & 0.02037 & 0.00104 & 0.29328 & 3.14016 & 0.0018 & 0.00009 & 3.14016 \\
\hline 0.34219 & 0.01397 & 0.00071 & 0.34219 & 3.18266 & 0.00495 & 0.00025 & 3.18266 \\
\hline 0.38969 & 0.00275 & 0.00014 & 0.38969 & 3.22516 & 0.00698 & 0.00036 & 3.22516 \\
\hline 0.43688 & -0.00282 & -0.00014 & 0.43687 & 3.26734 & 0.00565 & 0.00029 & 3.26734 \\
\hline 0.48422 & -0.0034 & -0.00017 & 0.48422 & 3.30984 & 0.00608 & 0.00031 & 3.30984 \\
\hline 0.53094 & -0.00115 & -0.00006 & 0.53094 & 3.35234 & 0.00662 & 0.00034 & 3.35234 \\
\hline 0.57641 & 0.00009 & 0 & 0.57641 & 3.39469 & 0.004 & 0.0002 & 3.39469 \\
\hline 0.62078 & -0.00198 & -0.0001 & 0.62078 & 3.43656 & 0.00273 & 0.00014 & 3.43656 \\
\hline 0.66563 & -0.00099 & -0.00005 & 0.66563 & 3.47891 & 0.00261 & 0.00013 & 3.47891 \\
\hline 0.71078 & 0.00014 & 0.00001 & 0.71078 & 3.52172 & 0.00318 & 0.00016 & 3.52172 \\
\hline 0.75547 & 0.00066 & 0.00003 & 0.75547 & 3.56437 & 0.00467 & 0.00024 & 3.56437 \\
\hline 0.79937 & 0.00069 & 0.00004 & 0.79938 & 3.60687 & 0.00236 & 0.00012 & 3.60687 \\
\hline 0.84312 & -0.00121 & -0.00006 & 0.84312 & 3.64906 & -0.00015 & -0.00001 & 3.64906 \\
\hline 0.88688 & 0.00152 & 0.00008 & 0.88688 & 3.69078 & 0.00479 & 0.00024 & 3.69078 \\
\hline 0.93047 & 0.00333 & 0.00017 & 0.93047 & 3.7325 & 0.00503 & 0.00026 & 3.7325 \\
\hline 0.97422 & 0.00187 & 0.0001 & 0.97422 & 3.77469 & 0.00489 & 0.00025 & 3.77469 \\
\hline 1.01812 & 0.0026 & 0.00013 & 1.01812 & 3.81734 & 0.00815 & 0.00041 & 3.81734 \\
\hline 1.06187 & 0.00149 & 0.00008 & 1.06187 & 3.86031 & 0.00697 & 0.00035 & 3.86031 \\
\hline 1.10516 & 0.00208 & 0.00011 & 1.10516 & 3.90266 & 0.00455 & 0.00023 & 3.90266 \\
\hline 1.14781 & -0.0001 & -0.00001 & 1.14781 & 3.945 & 0.00251 & 0.00013 & 3.945 \\
\hline 1.19047 & -0.00261 & -0.00013 & 1.19047 & 3.9875 & 0.00291 & 0.00015 & 3.9875 \\
\hline 1.2325 & 0.00046 & 0.00002 & 1.2325 & 4.03 & 0.00631 & 0.00032 & 4.03 \\
\hline 1.27469 & 0.00267 & 0.00014 & 1.27469 & 4.07219 & 0.00399 & 0.0002 & 4.07219 \\
\hline 1.3175 & 0.00157 & 0.00008 & 1.3175 & 4.115 & 0.00242 & 0.00012 & 4.115 \\
\hline 1.36031 & 0.00509 & 0.00026 & 1.36031 & 4.15781 & 0.00503 & 0.00026 & 4.15781 \\
\hline 1.40313 & 0.009 & 0.00046 & 1.40313 & 4.20016 & 0.00277 & 0.00014 & 4.20016 \\
\hline 1.44562 & 0.00493 & 0.00025 & 1.44562 & 4.24234 & 0.005 & 0.00025 & 4.24234 \\
\hline 1.48828 & 0.00314 & 0.00016 & 1.48828 & 4.28453 & 0.00616 & 0.00031 & 4.28453 \\
\hline 1.53094 & 0.00655 & 0.00033 & 1.53094 & 4.32641 & 0.00267 & 0.00014 & 4.32641 \\
\hline 1.57359 & 0.00414 & 0.00021 & 1.57359 & 4.36766 & 0.00363 & 0.00018 & 4.36766 \\
\hline 1.61672 & 0.00263 & 0.00013 & 1.61672 & 4.40906 & 0.0021 & 0.00011 & 4.40906 \\
\hline 1.65984 & 0.00343 & 0.00017 & 1.65984 & 4.45125 & 0.00284 & 0.00014 & 4.45125 \\
\hline 1.70234 & 0.00114 & 0.00006 & 1.70234 & 4.49344 & 0.00546 & 0.00028 & 4.49344 \\
\hline 1.74437 & 0.00069 & 0.00004 & 1.74437 & 4.53656 & 0.00547 & 0.00028 & 4.53656 \\
\hline 1.78688 & 0.00203 & 0.0001 & 1.78688 & 4.58047 & 0.00671 & 0.00034 & 4.58047 \\
\hline 1.82891 & 0.00378 & 0.00019 & 1.82891 & 4.62359 & 0.0039 & 0.0002 & 4.62359 \\
\hline 1.87062 & 0.00239 & 0.00012 & 1.87062 & 4.66563 & 0.00052 & 0.00003 & 4.66563 \\
\hline 1.9125 & 0.00064 & 0.00003 & 1.9125 & 4.70797 & 0.00085 & 0.00004 & 4.70797 \\
\hline 1.955 & 0.00544 & 0.00028 & 1.955 & 4.75125 & 0.00335 & 0.00017 & 4.75125 \\
\hline 1.99734 & 0.00325 & 0.00017 & 1.99734 & 4.79437 & 0.00621 & 0.00032 & 4.79437 \\
\hline 2.03969 & 0.0006 & 0.00003 & 2.03969 & 4.83656 & 0.00445 & 0.00023 & 4.83656 \\
\hline 2.08219 & 0.00377 & 0.00019 & 2.08219 & 4.87859 & 0.00092 & 0.00005 & 4.87859 \\
\hline 2.125 & -0.00004 & 0 & 2.125 & 4.92047 & 0.00251 & 0.00013 & 4.92047 \\
\hline 2.16719 & $\begin{array}{c}-0.0018 \\
\end{array}$ & -0.00009 & 2.16719 & 4.96219 & 0.00367 & 0.00019 & 4.96219 \\
\hline 2.20984 & 0.00142 & 0.00007 & 2.20984 & 5.00375 & 0.00475 & 0.00024 & 5.00375 \\
\hline 2.25297 & 0.00419 & 0.00021 & 2.25297 & 5.04562 & 0.00406 & 0.00021 & 5.04562 \\
\hline 2.29563 & 0.00792 & 0.0004 & 2.29563 & 5.08797 & 0.00456 & 0.00023 & 5.08797 \\
\hline 2.33813 & 0.00469 & 0.00024 & 2.33813 & 5.13 & 0.00626 & 0.00032 & 5.13 \\
\hline 2.38078 & 0.00535 & 0.00027 & 2.38078 & 5.17172 & 0.00599 & 0.00031 & 5.17172 \\
\hline 2.42266 & 0.00628 & 0.00032 & 2.42266 & 5.21422 & 0.00781 & 0.0004 & 5.21422 \\
\hline 2.46422 & 0.00093 & 0.00005 & 2.46422 & 5.25719 & 0.00851 & 0.00043 & 5.25719 \\
\hline 2.50578 & 0.00219 & 0.00011 & 2.50578 & 5.30031 & 0.0072 & 0.00037 & 5.30031 \\
\hline 2.54797 & 0.0039 & 0.0002 & 2.54797 & 5.34328 & 0.00426 & 0.00022 & 5.34328 \\
\hline 2.59047 & 0.0035 & 0.00018 & 2.59047 & 5.38609 & 0.00006 & 0 & 5.38609 \\
\hline 2.63281 & 0.00384 & 0.0002 & 2.63281 & 5.42891 & 0.00139 & 0.00007 & 5.42891 \\
\hline 2.67516 & 0.00311 & 0.00016 & 2.67516 & 5.47141 & 0.0042 & 0.00021 & 5.47141 \\
\hline 2.71766 & 0.00514 & 0.00026 & 2.71766 & 5.51375 & 0.0038 & 0.00019 & 5.51375 \\
\hline
\end{tabular}




\begin{tabular}{|c|c|c|c|}
\hline $\begin{array}{l}\text { Extension } \\
\text { (mm) }\end{array}$ & $\begin{array}{l}\text { Load } \\
\text { (N) }\end{array}$ & $\begin{array}{c}\text { Compressive stress } \\
\text { (MPa) }\end{array}$ & $\underset{[\mathrm{mm} / \mathrm{mm})}{\text { Compressive strain }}$ \\
\hline 5.59688 & 0.00386 & 0.0002 & 5.59688 \\
\hline 5.63828 & 0.00423 & 0.00022 & 5.63828 \\
\hline 5.68047 & 0.00643 & 0.00033 & 5.68047 \\
\hline 5.72281 & 0.00611 & 0.00031 & 5.72281 \\
\hline 5.76516 & 0.00466 & 0.00024 & 5.76516 \\
\hline 5.80734 & 0.0045 & 0.00023 & 5.80734 \\
\hline 5.84984 & 0.00168 & 0.00009 & 5.84984 \\
\hline 5.89234 & 0.0009 & 0.00005 & 5.89234 \\
\hline 5.93469 & 0.00401 & 0.0002 & 5.93469 \\
\hline 5.97719 & 0.00395 & 0.0002 & 5.97719 \\
\hline 6.02031 & 0.00554 & 0.00028 & 6.02031 \\
\hline 6.06297 & 0.00628 & 0.00032 & 6.06297 \\
\hline 6.10531 & 0.00702 & 0.00036 & 6.10531 \\
\hline 6.1475 & 0.00775 & 0.00039 & 6.1475 \\
\hline 6.18969 & 0.0063 & 0.00032 & 6.18969 \\
\hline 6.23156 & 0.00809 & 0.00041 & 6.23156 \\
\hline 6.27375 & 0.00605 & 0.00031 & 6.27375 \\
\hline 6.31594 & 0.00204 & 0.0001 & 6.31594 \\
\hline 6.35813 & 0.00347 & 0.00018 & 6.35813 \\
\hline 6.40047 & 0.00522 & 0.00027 & 6.40047 \\
\hline 6.44297 & 0.00813 & 0.00041 & 6.44297 \\
\hline 6.485 & 0.00788 & 0.0004 & 6.485 \\
\hline 6.52688 & 0.00419 & 0.00021 & 6.52688 \\
\hline 6.56922 & 0.00457 & 0.00023 & 6.56922 \\
\hline 6.61234 & 0.00522 & 0.00027 & 6.61234 \\
\hline 6.65547 & 0.00671 & 0.00034 & 6.65547 \\
\hline 6.69812 & 0.00716 & 0.00036 & 6.69812 \\
\hline 6.74031 & 0.00391 & 0.0002 & 6.74031 \\
\hline 6.78281 & 0.00409 & 0.00021 & 6.78281 \\
\hline 6.82453 & 0.00353 & 0.00018 & 6.82453 \\
\hline 6.86594 & 0.00237 & 0.00012 & 6.86594 \\
\hline 6.90766 & 0.00447 & 0.00023 & 6.90766 \\
\hline 6.95 & 0.00675 & 0.00034 & 6.95 \\
\hline 6.99234 & 0.00694 & 0.00035 & 6.99234 \\
\hline 7.035 & 0.00691 & 0.00035 & 7.035 \\
\hline 7.07781 & 0.00531 & 0.00027 & 7.07781 \\
\hline 7.12047 & 0.00719 & 0.00037 & 7.12047 \\
\hline 7.16297 & 0.00822 & 0.00042 & 7.16297 \\
\hline 7.20563 & 0.00482 & 0.00025 & 7.20563 \\
\hline 7.24844 & 0.00345 & 0.00018 & 7.24844 \\
\hline 7.29109 & 0.00641 & 0.00033 & 7.29109 \\
\hline 7.33328 & 0.00503 & 0.00026 & 7.33328 \\
\hline 7.37547 & 0.00128 & 0.00007 & 7.37547 \\
\hline 7.41781 & 0.00493 & 0.00025 & 7.41781 \\
\hline 7.45984 & 0.00806 & 0.00041 & 7.45984 \\
\hline 7.50156 & 0.00464 & 0.00024 & 7.50156 \\
\hline 7.54406 & 0.00291 & 0.00015 & 7.54406 \\
\hline 7.58625 & 0.00848 & 0.00043 & 7.58625 \\
\hline 7.62844 & 0.01036 & 0.00053 & 7.62844 \\
\hline 7.67063 & 0.00611 & 0.00031 & 7.67063 \\
\hline 7.71266 & 0.00633 & 0.00032 & 7.71266 \\
\hline 7.75438 & 0.008 & 0.00041 & 7.75438 \\
\hline 7.79656 & 0.00319 & 0.00016 & 7.79656 \\
\hline 7.83969 & 0.00171 & 0.00009 & 7.83969 \\
\hline 7.88266 & 0.00442 & 0.00022 & 7.88266 \\
\hline 7.92531 & 0.00529 & 0.00027 & 7.92531 \\
\hline 7.9675 & 0.00469 & 0.00024 & 7.9675 \\
\hline 8.01 & 0.00463 & 0.00024 & 8.01 \\
\hline 8.05234 & 0.00793 & 0.0004 & 8.05234 \\
\hline 8.09406 & 0.00959 & 0.00049 & 8.09406 \\
\hline 8.13562 & 0.00661 & 0.00034 & 8.13562 \\
\hline 8.17781 & 0.00826 & 0.00042 & 8.17781 \\
\hline 8.22031 & 0.00739 & 0.00038 & 8.22031 \\
\hline 8.26266 & 0.00381 & 0.00019 & 8.26266 \\
\hline 8.305 & 0.0051 & 0.00026 & 8.305 \\
\hline
\end{tabular}

\begin{tabular}{|c|c|c|c|}
\hline $\begin{array}{l}\text { Extension } \\
\text { (mm) }\end{array}$ & $\begin{array}{l}\text { Load } \\
\text { (N) }\end{array}$ & $\begin{array}{c}\text { Compressive stress } \\
\text { (MPa] }\end{array}$ & $\begin{array}{c}\text { Compressive strain } \\
\text { [mm/mm] }\end{array}$ \\
\hline 8.39016 & 0.00715 & 0.00036 & 8.39016 \\
\hline 8.43219 & 0.00833 & 0.00042 & 8.43219 \\
\hline 8.47453 & 0.00445 & 0.00023 & 8.47453 \\
\hline 8.51719 & 0.00572 & 0.00029 & 8.51719 \\
\hline 8.55969 & 0.00685 & 0.00035 & 8.55969 \\
\hline 8.6025 & 0.0069 & 0.00035 & 8.6025 \\
\hline 8.645 & 0.00833 & 0.00042 & 8.645 \\
\hline 8.68703 & 0.00476 & 0.00024 & 8.68703 \\
\hline 8.72828 & 0.00166 & 0.00008 & 8.72828 \\
\hline 8.76984 & -0.00024 & -0.00001 & 8.76984 \\
\hline 8.8125 & 0.0001 & 0.00001 & 8.8125 \\
\hline 8.855 & 0.00576 & 0.00029 & 8.855 \\
\hline 8.89719 & 0.00638 & 0.00032 & 8.89719 \\
\hline 8.93953 & 0.00503 & 0.00026 & 8.93953 \\
\hline 8.98234 & 0.0081 & 0.00041 & 8.98234 \\
\hline 9.02516 & 0.00997 & 0.00051 & 9.02516 \\
\hline 9.0675 & 0.01 & 0.00051 & 9.0675 \\
\hline 9.10984 & 0.00896 & 0.00046 & 9.10984 \\
\hline 9.1525 & 0.00824 & 0.00042 & 9.1525 \\
\hline 9.19531 & 0.00644 & 0.00033 & 9.19531 \\
\hline 9.23766 & 0.00415 & 0.00021 & 9.23766 \\
\hline 9.28 & 0.00343 & 0.00017 & 9.28 \\
\hline 9.32187 & 0.00459 & 0.00023 & 9.32187 \\
\hline 9.36344 & 0.00767 & 0.00039 & 9.36344 \\
\hline 9.40531 & 0.00853 & 0.00043 & 9.40531 \\
\hline 9.44766 & 0.00827 & 0.00042 & 9.44766 \\
\hline 9.49 & 0.00784 & 0.0004 & 9.49 \\
\hline 9.53234 & 0.00806 & 0.00041 & 9.53234 \\
\hline 9.57516 & 0.00923 & 0.00047 & 9.57516 \\
\hline 9.61797 & 0.00904 & 0.00046 & 9.61797 \\
\hline 9.66031 & 0.00768 & 0.00039 & 9.66031 \\
\hline 9.70266 & 0.00732 & 0.00037 & 9.70266 \\
\hline 9.74547 & 0.00555 & 0.00028 & 9.74547 \\
\hline 9.78844 & 0.00539 & 0.00027 & 9.78844 \\
\hline 9.83078 & 0.0074 & 0.00038 & 9.83078 \\
\hline 9.87297 & 0.00657 & 0.00033 & 9.87297 \\
\hline 9.91516 & 0.00457 & 0.00023 & 9.91516 \\
\hline 9.95672 & 0.00505 & 0.00026 & 9.95672 \\
\hline 9.99828 & 0.08635 & 0.0044 & 9.99828 \\
\hline 10.04031 & 0.44832 & 0.02283 & 10.04031 \\
\hline 10.08281 & 1.13186 & 0.05765 & 10.08281 \\
\hline 10.12516 & 2.07156 & 0.1055 & 10.12516 \\
\hline 10.1675 & 3.17536 & 0.16172 & 10.1675 \\
\hline 10.21031 & 4.37688 & 0.22291 & 10.21031 \\
\hline 10.25297 & 5.63598 & 0.28704 & 10.25297 \\
\hline 10.29547 & 6.81618 & 0.34715 & 10.29547 \\
\hline 10.33813 & 7.65347 & 0.38979 & 10.33813 \\
\hline 10.38094 & 8.16077 & 0.41562 & 10.38094 \\
\hline 10.42359 & 8.47268 & 0.43151 & 10.42359 \\
\hline 10.46594 & 8.86698 & 0.45159 & 10.46594 \\
\hline 10.50797 & 9.60629 & 0.48924 & 10.50797 \\
\hline 10.54984 & 10.63737 & 0.54176 & 10.54984 \\
\hline 10.59125 & 11.87639 & 0.60486 & 10.59125 \\
\hline 10.63281 & 13.25089 & 0.67486 & 10.63281 \\
\hline 10.675 & 14.72268 & 0.74982 & 10.675 \\
\hline 10.71734 & 16.26825 & 0.82854 & 10.71734 \\
\hline 10.75969 & 17.86912 & 0.91007 & 10.75969 \\
\hline 10.80219 & 19.51544 & 0.99391 & 10.80219 \\
\hline 10.84516 & 21.2065 & 1.08004 & 10.84516 \\
\hline 10.88797 & 22.94672 & 1.16867 & 10.88797 \\
\hline 10.93031 & 24.72732 & 1.25935 & 10.93031 \\
\hline 10.97281 & 26.54093 & 1.35172 & 10.97281 \\
\hline 11.01547 & 28.39317 & 1.44605 & 11.01547 \\
\hline 11.05781 & 30.28424 & 1.54236 & 11.05781 \\
\hline 11.09984 & 32.21847 & 1.64087 & 11.09984 \\
\hline
\end{tabular}




\begin{tabular}{|c|c|c|c|}
\hline $\begin{array}{l}\text { Extension } \\
\text { [mm] }\end{array}$ & $\begin{array}{l}\text { Load } \\
\text { [N] }\end{array}$ & $\begin{array}{c}\text { Compressive stress } \\
\text { [MPa] }\end{array}$ & $\begin{array}{c}\text { Compressive strain } \\
{[\mathrm{mm} / \mathrm{mm}]}\end{array}$ \\
\hline 11.18375 & 36.27646 & 1.84754 & 11.18375 \\
\hline 11.22547 & 38.4143 & 1.95642 & 11.22547 \\
\hline 11.26734 & 40.6481 & 2.07019 & 11.26734 \\
\hline 11.30984 & 42.97743 & 2.18882 & 11.30984 \\
\hline 11.3525 & 45.42378 & 2.31341 & 11.3525 \\
\hline 11.39469 & 48.00034 & 2.44464 & 11.39469 \\
\hline 11.43703 & 50.68847 & 2.58154 & 11.43703 \\
\hline 11.47969 & 53.49343 & 2.7244 & 11.47969 \\
\hline 11.52234 & 56.44195 & 2.87456 & 11.52234 \\
\hline 11.56469 & 59.52526 & 3.0316 & 11.56469 \\
\hline 11.60719 & 62.72189 & 3.1944 & 11.60719 \\
\hline 11.64984 & 66.06515 & 3.36467 & 11.64985 \\
\hline 11.69281 & 69.59782 & 3.54459 & 11.69281 \\
\hline 11.73531 & 73.33073 & 3.7347 & 11.73531 \\
\hline 11.7775 & 77.27135 & 3.9354 & 11.7775 \\
\hline 11.81922 & 81.39272 & 4.1453 & 11.81922 \\
\hline 11.86047 & 85.69207 & 4.36426 & 11.86047 \\
\hline 11.90219 & 90.18945 & 4.59331 & 11.90219 \\
\hline 11.94453 & 94.97472 & 4.83702 & 11.94453 \\
\hline 11.98672 & 100.11778 & 5.09896 & 11.98672 \\
\hline 12.02875 & 105.63795 & 5.3801 & 12.02875 \\
\hline 12.07141 & 111.54954 & 5.68117 & 12.07141 \\
\hline 12.11438 & 117.8905 & 6.00411 & 12.11437 \\
\hline 12.15687 & 124.70668 & 6.35126 & 12.15688 \\
\hline 12.19906 & 131.9967 & 6.72254 & 12.19906 \\
\hline 12.24156 & 139.74664 & 7.11724 & 12.24156 \\
\hline 12.28422 & 148.03667 & 7.53945 & 12.28422 \\
\hline 12.32656 & 156.9035 & 7.99103 & 12.32656 \\
\hline 12.36891 & 166.30673 & 8.46993 & 12.36891 \\
\hline 12.41141 & 176.27115 & 8.97742 & 12.41141 \\
\hline 12.45281 & 186.85536 & 9.51647 & 12.45281 \\
\hline 12.49391 & 198.00252 & 10.08419 & 12.49391 \\
\hline 12.53563 & 209.71548 & 10.68072 & 12.53563 \\
\hline 12.57797 & 222.19504 & 11.3163 & 12.57797 \\
\hline 12.61984 & 235.61417 & 11.99973 & 12.61984 \\
\hline 12.66187 & 249.93517 & 12.72909 & 12.66187 \\
\hline 12.70406 & 265.24774 & 13.50896 & 12.70406 \\
\hline 12.74656 & 281.70694 & 14.34722 & 12.74656 \\
\hline 12.78906 & 299.42722 & 15.2497 & 12.78906 \\
\hline 12.83125 & 318.42993 & 16.2175 & 12.83125 \\
\hline 12.87359 & 338.69714 & 17.2497 & 12.87359 \\
\hline 12.91594 & 360.28778 & 18.34931 & 12.91594 \\
\hline 12.95813 & 383.27441 & 19.52001 & 12.95813 \\
\hline 13 & 407.7056 & 20.76427 & 13 \\
\hline 13.04187 & 433.50879 & 22.07842 & 13.04188 \\
\hline 13.07484 & 455.0961 & 23.17785 & 13.07484 \\
\hline
\end{tabular}


Table D.12 PBS-Soaked Scaffolds Specimen 4 Raw Compression Data

\begin{tabular}{|c|c|c|c|c|c|c|c|}
\hline $\begin{array}{c}\text { Extension } \\
\text { (mm) }\end{array}$ & $\begin{array}{c}\text { Load } \\
\text { (N) }\end{array}$ & $\begin{array}{c}\text { Compressive stress } \\
\text { (MPa] }\end{array}$ & $\begin{array}{c}\text { Compressive strain } \\
{[\mathrm{mm} / \mathrm{mm}]}\end{array}$ & $\begin{array}{c}\text { Extension } \\
\text { (mm) }\end{array}$ & $\begin{array}{c}\text { Load } \\
\text { (N) }\end{array}$ & $\begin{array}{c}\text { Compressive stress } \\
\text { (MPa) }\end{array}$ & $\begin{array}{c}\text { Compressive strain } \\
\text { [mmimm] }\end{array}$ \\
\hline 0 & 0.00371 & 0.00019 & 0 & 2.71703 & 0.00398 & 0.0002 & 0.54341 \\
\hline 0.00281 & 0.00307 & 0.00016 & 0.00056 & 2.75953 & 0.00337 & 0.00017 & 0.55191 \\
\hline 0.02375 & 0.0198 & 0.00101 & 0.00475 & 2.80234 & 0.00434 & 0.00022 & 0.56047 \\
\hline 0.06563 & 0.05808 & 0.00296 & 0.01313 & 2.84469 & 0.00542 & 0.00028 & 0.56894 \\
\hline 0.11156 & 0.08096 & 0.00412 & 0.02231 & 2.88687 & 0.00421 & 0.00021 & 0.57737 \\
\hline 0.15328 & 0.06476 & 0.0033 & 0.03066 & 2.92969 & 0.00315 & 0.00016 & 0.58594 \\
\hline 0.19609 & 0.03664 & 0.00187 & 0.03922 & 2.97234 & 0.00338 & 0.00017 & 0.59447 \\
\hline 0.24484 & 0.02355 & 0.0012 & 0.04897 & 3.01469 & 0.0045 & 0.00023 & 0.60294 \\
\hline 0.29531 & 0.0209 & 0.00106 & 0.05906 & 3.05672 & 0.00562 & 0.00029 & 0.61134 \\
\hline 0.34391 & 0.01528 & 0.00078 & 0.06878 & 3.09828 & 0.0062 & 0.00032 & 0.61966 \\
\hline 0.39094 & 0.00543 & 0.00028 & 0.07819 & 3.14 & 0.00651 & 0.00033 & 0.628 \\
\hline 0.43812 & -0.00126 & -0.00006 & 0.08762 & 3.1825 & 0.00672 & 0.00034 & 0.6365 \\
\hline 0.48547 & -0.0014 & -0.00007 & 0.09709 & 3.225 & 0.0062 & 0.00032 & 0.645 \\
\hline 0.53219 & -0.00136 & -0.00007 & 0.10644 & 3.26734 & 0.00589 & 0.0003 & 0.65347 \\
\hline 0.57734 & -0.00131 & -0.00007 & 0.11547 & 3.30969 & 0.00616 & 0.00031 & 0.66194 \\
\hline 0.62156 & -0.00095 & -0.00005 & 0.12431 & 3.35219 & 0.0079 & 0.0004 & 0.67044 \\
\hline 0.66625 & 0.00127 & 0.00006 & 0.13325 & 3.39438 & 0.00637 & 0.00032 & 0.67888 \\
\hline 0.71141 & 0.00406 & 0.00021 & 0.14228 & 3.43641 & 0.00334 & 0.00017 & 0.68728 \\
\hline 0.75594 & 0.00479 & 0.00024 & 0.15119 & 3.47875 & 0.00344 & 0.00018 & 0.69575 \\
\hline 0.79984 & 0.00219 & 0.00011 & 0.15997 & 3.52172 & 0.00593 & 0.0003 & 0.70434 \\
\hline 0.84359 & 0.00181 & 0.00009 & 0.16872 & 3.56406 & 0.00643 & 0.00033 & 0.71281 \\
\hline 0.8875 & 0.00332 & 0.00017 & 0.1775 & 3.60594 & 0.0052 & 0.00026 & 0.72119 \\
\hline 0.93094 & 0.00412 & 0.00021 & 0.18619 & 3.64813 & 0.00388 & 0.0002 & 0.72963 \\
\hline 0.97438 & 0.00259 & 0.00013 & 0.19488 & 3.69094 & 0.00402 & 0.0002 & 0.73819 \\
\hline 1.01844 & 0.00288 & 0.00015 & 0.20369 & 3.73344 & 0.0046 & 0.00023 & 0.74669 \\
\hline 1.06219 & 0.00338 & 0.00017 & 0.21244 & 3.77531 & 0.00771 & 0.00039 & 0.75506 \\
\hline 1.10531 & 0.00244 & 0.00012 & 0.22106 & 3.8175 & 0.01041 & 0.00053 & 0.7635 \\
\hline 1.14797 & 0.00108 & 0.00005 & 0.22959 & 3.86 & 0.0093 & 0.00047 & 0.772 \\
\hline 1.19047 & 0.00101 & 0.00005 & 0.23809 & 3.90234 & 0.00587 & 0.0003 & 0.78047 \\
\hline 1.2325 & 0.00206 & 0.00011 & 0.2465 & 3.94484 & 0.00501 & 0.00025 & 0.78897 \\
\hline 1.27484 & 0.00119 & 0.00006 & 0.25497 & 3.98719 & 0.00636 & 0.00032 & 0.79744 \\
\hline 1.3175 & 0.00097 & 0.00005 & 0.2635 & 4.02969 & 0.00721 & 0.00037 & 0.80594 \\
\hline 1.36016 & 0.00476 & 0.00024 & 0.27203 & 4.07219 & 0.00656 & 0.00033 & 0.81444 \\
\hline 1.4025 & 0.00755 & 0.00038 & 0.2805 & 4.115 & 0.00603 & 0.00031 & 0.823 \\
\hline 1.44516 & 0.00543 & 0.00028 & 0.28903 & 4.1575 & 0.00572 & 0.00029 & 0.8315 \\
\hline 1.48828 & 0.00272 & 0.00014 & 0.29766 & 4.19984 & 0.00598 & 0.0003 & 0.83997 \\
\hline 1.53109 & 0.00315 & 0.00016 & 0.30622 & 4.24203 & 0.00725 & 0.00037 & 0.84841 \\
\hline 1.57375 & 0.00557 & 0.00028 & 0.31475 & 4.28375 & 0.00777 & 0.0004 & 0.85675 \\
\hline 1.61688 & 0.00598 & 0.0003 & 0.32338 & 4.325 & 0.00743 & 0.00038 & 0.865 \\
\hline 1.65984 & 0.00647 & 0.00033 & 0.33197 & 4.36672 & 0.00682 & 0.00035 & 0.87334 \\
\hline 1.7025 & 0.00528 & 0.00027 & 0.3405 & 4.40969 & 0.00761 & 0.00039 & 0.88194 \\
\hline 1.74469 & 0.00273 & 0.00014 & 0.34894 & 4.45281 & 0.00926 & 0.00047 & 0.89056 \\
\hline 1.78672 & 0.00102 & 0.00005 & 0.35734 & 4.49516 & 0.00914 & 0.00047 & 0.89903 \\
\hline 1.82844 & 0.00055 & 0.00003 & 0.36569 & 4.53719 & 0.00742 & 0.00038 & 0.90744 \\
\hline 1.86984 & 0.00193 & 0.0001 & 0.37397 & 4.58 & 0.00562 & 0.00029 & 0.916 \\
\hline 1.91187 & 0.00558 & 0.00028 & 0.38237 & 4.62328 & 0.00575 & 0.00029 & 0.92466 \\
\hline 1.95453 & 0.00918 & 0.00047 & 0.39091 & 4.66578 & 0.00569 & 0.00029 & 0.93316 \\
\hline 1.99703 & 0.00982 & 0.0005 & 0.39941 & 4.70797 & 0.00296 & 0.00015 & 0.94159 \\
\hline 2.03922 & 0.00887 & 0.00045 & 0.40784 & 4.75078 & 0.00262 & 0.00013 & 0.95016 \\
\hline 2.08219 & 0.00842 & 0.00043 & 0.41644 & 4.79359 & 0.00492 & 0.00025 & 0.95872 \\
\hline 2.12531 & 0.00766 & 0.00039 & 0.42506 & 4.83594 & 0.00527 & 0.00027 & 0.96719 \\
\hline 2.16797 & 0.00559 & 0.00028 & 0.43359 & 4.87828 & 0.00481 & 0.00025 & 0.97566 \\
\hline 2.21031 & 0.00291 & 0.00015 & 0.44206 & 4.92016 & 0.00502 & 0.00026 & 0.98403 \\
\hline 2.25313 & 0.00124 & 0.00006 & 0.45063 & 4.96141 & 0.00686 & 0.00035 & 0.99228 \\
\hline 2.29578 & 0.0012 & 0.00006 & 0.45916 & 5.00219 & 0.00644 & 0.00033 & 1.00044 \\
\hline 2.33813 & 0.00187 & 0.0001 & 0.46763 & 5.04437 & 0.00666 & 0.00034 & 1.00888 \\
\hline 2.38031 & 0.00381 & 0.00019 & 0.47606 & 5.0875 & 0.01054 & 0.00054 & 1.0175 \\
\hline 2.42234 & 0.00541 & 0.00028 & 0.48447 & 5.13047 & 0.00997 & 0.00051 & 1.02609 \\
\hline 2.46406 & 0.00497 & 0.00025 & 0.49281 & 5.17281 & 0.00571 & 0.00029 & 1.03456 \\
\hline 2.50563 & 0.00519 & 0.00026 & 0.50113 & 5.21547 & 0.00219 & 0.00011 & 1.04309 \\
\hline 2.5475 & 0.00643 & 0.00033 & 0.5095 & 5.25813 & 0.00363 & 0.00019 & 1.05163 \\
\hline 2.58984 & 0.00623 & 0.00032 & 0.51797 & 5.30062 & 0.00489 & 0.00025 & 1.06012 \\
\hline 2.6325 & 0.0068 & 0.00035 & 0.5265 & 5.34297 & 0.0037 & 0.00019 & 1.06859 \\
\hline 2.67484 & 0.00551 & 0.00028 & 0.53497 & 5.38578 & 0.00381 & 0.00019 & 1.07716 \\
\hline
\end{tabular}




\begin{tabular}{|c|c|c|c|}
\hline $\begin{array}{c}\text { Extension } \\
\text { (mm) }\end{array}$ & $\begin{array}{l}\text { Load } \\
\text { (N) }\end{array}$ & $\begin{array}{c}\text { Compressive stress } \\
\text { (MPa] }\end{array}$ & $\begin{array}{c}\text { Compressive strain } \\
\text { [mmimm })\end{array}$ \\
\hline 5.47109 & 0.00587 & 0.0003 & 1.09422 \\
\hline 5.51312 & 0.00609 & 0.00031 & 1.10263 \\
\hline 5.555 & 0.00587 & 0.0003 & 1.111 \\
\hline 5.59656 & 0.00595 & 0.0003 & 1.11931 \\
\hline 5.63797 & 0.00531 & 0.00027 & 1.12759 \\
\hline 5.67984 & 0.00672 & 0.00034 & 1.13597 \\
\hline 5.72203 & 0.00795 & 0.0004 & 1.14441 \\
\hline 5.76438 & 0.0089 & 0.00045 & 1.15288 \\
\hline 5.80641 & 0.00844 & 0.00043 & 1.16128 \\
\hline 5.84938 & 0.0069 & 0.00035 & 1.16988 \\
\hline 5.89281 & 0.00771 & 0.00039 & 1.17856 \\
\hline 5.93547 & 0.00865 & 0.00044 & 1.18709 \\
\hline 5.97766 & 0.00616 & 0.00031 & 1.19553 \\
\hline 6.02016 & 0.00375 & 0.00019 & 1.20403 \\
\hline 6.06297 & 0.00462 & 0.00024 & 1.21259 \\
\hline 6.10531 & 0.00662 & 0.00034 & 1.22106 \\
\hline 6.1475 & 0.00573 & 0.00029 & 1.2295 \\
\hline 6.18984 & 0.00404 & 0.00021 & 1.23797 \\
\hline 6.23156 & 0.0061 & 0.00031 & 1.24631 \\
\hline 6.27344 & 0.00854 & 0.00043 & 1.25469 \\
\hline 6.31578 & 0.00867 & 0.00044 & 1.26316 \\
\hline 6.35797 & 0.00758 & 0.00039 & 1.27159 \\
\hline 6.4 & 0.00681 & 0.00035 & 1.28 \\
\hline 6.44203 & 0.0063 & 0.00032 & 1.28841 \\
\hline 6.48391 & 0.00578 & 0.00029 & 1.29678 \\
\hline 6.52609 & 0.00658 & 0.00034 & 1.30522 \\
\hline 6.56891 & 0.00832 & 0.00042 & 1.31378 \\
\hline 6.6125 & 0.00873 & 0.00044 & 1.3225 \\
\hline 6.65547 & 0.00741 & 0.00038 & 1.33109 \\
\hline 6.69797 & 0.00506 & 0.00026 & 1.33959 \\
\hline 6.74031 & 0.00465 & 0.00024 & 1.34806 \\
\hline 6.78266 & 0.00569 & 0.00029 & 1.35653 \\
\hline 6.82453 & 0.00562 & 0.00029 & 1.36491 \\
\hline 6.86594 & 0.00521 & 0.00027 & 1.37319 \\
\hline 6.90781 & 0.00589 & 0.0003 & 1.38156 \\
\hline 6.95031 & 0.00667 & 0.00034 & 1.39006 \\
\hline 6.9925 & 0.00647 & 0.00033 & 1.3985 \\
\hline 7.03469 & 0.00607 & 0.00031 & 1.40694 \\
\hline 7.0775 & 0.00746 & 0.00038 & 1.4155 \\
\hline 7.12031 & 0.00766 & 0.00039 & 1.42406 \\
\hline 7.16281 & 0.00746 & 0.00038 & 1.43256 \\
\hline 7.20531 & 0.00736 & 0.00037 & 1.44106 \\
\hline 7.24813 & 0.00843 & 0.00043 & 1.44963 \\
\hline 7.29094 & 0.00744 & 0.00038 & 1.45819 \\
\hline 7.33328 & 0.00281 & 0.00014 & 1.46666 \\
\hline 7.37531 & 0.0019 & 0.0001 & 1.47506 \\
\hline 7.41766 & 0.0034 & 0.00017 & 1.48353 \\
\hline 7.45969 & 0.00385 & 0.0002 & 1.49194 \\
\hline 7.50141 & 0.00511 & 0.00026 & 1.50028 \\
\hline 7.54344 & 0.0062 & 0.00032 & 1.50869 \\
\hline 7.58563 & 0.00668 & 0.00034 & 1.51713 \\
\hline 7.62781 & 0.00597 & 0.0003 & 1.52556 \\
\hline 7.67031 & 0.00762 & 0.00039 & 1.53406 \\
\hline 7.71266 & 0.00991 & 0.0005 & 1.54253 \\
\hline 7.75453 & 0.00766 & 0.00039 & 1.55091 \\
\hline 7.79672 & 0.00494 & 0.00025 & 1.55934 \\
\hline 7.83969 & 0.0049 & 0.00025 & 1.56794 \\
\hline 7.88266 & 0.00564 & 0.00029 & 1.57653 \\
\hline 7.92531 & 0.00581 & 0.0003 & 1.58506 \\
\hline 7.9675 & 0.00447 & 0.00023 & 1.5935 \\
\hline 8.01 & 0.00483 & 0.00025 & 1.602 \\
\hline 8.05234 & 0.00563 & 0.00029 & 1.61047 \\
\hline 8.09391 & 0.0044 & 0.00022 & 1.61878 \\
\hline 8.13516 & 0.00323 & 0.00016 & 1.62703 \\
\hline
\end{tabular}

\begin{tabular}{|c|c|c|c|}
\hline $\begin{array}{l}\text { Extension } \\
\text { (mm) }\end{array}$ & $\begin{array}{l}\text { Load } \\
\text { (N) }\end{array}$ & \begin{tabular}{|c|} 
Compressive stress \\
(MPa)
\end{tabular} & $\begin{array}{c}\text { Compressive strain } \\
\text { (mm/mm) }\end{array}$ \\
\hline 8.17734 & 0.00532 & 0.00027 & 1.63547 \\
\hline 8.22 & 0.00955 & 0.00049 & 1.644 \\
\hline 8.2625 & 0.00848 & 0.00043 & 1.6525 \\
\hline 8.30484 & 0.00355 & 0.00018 & 1.66097 \\
\hline 8.34734 & 0.00518 & 0.00026 & 1.66947 \\
\hline 8.39 & 0.00858 & 0.00044 & 1.678 \\
\hline 8.43219 & 0.0071 & 0.00036 & 1.68644 \\
\hline 8.47437 & 0.00593 & 0.0003 & 1.69488 \\
\hline 8.51703 & 0.00734 & 0.00037 & 1.70341 \\
\hline 8.55969 & 0.00789 & 0.0004 & 1.71194 \\
\hline 8.60203 & 0.00696 & 0.00035 & 1.72041 \\
\hline 8.64437 & 0.00684 & 0.00035 & 1.72888 \\
\hline 8.68656 & 0.00685 & 0.00035 & 1.73731 \\
\hline 8.72844 & 0.00477 & 0.00024 & 1.74569 \\
\hline 8.77047 & 0.00366 & 0.00019 & 1.75409 \\
\hline 8.8125 & 0.0045 & 0.00023 & 1.7625 \\
\hline 8.85484 & 0.00614 & 0.00031 & 1.77097 \\
\hline 8.89703 & 0.00662 & 0.00034 & 1.77941 \\
\hline 8.93953 & 0.00691 & 0.00035 & 1.78791 \\
\hline 8.98234 & 0.00781 & 0.0004 & 1.79647 \\
\hline 9.025 & 0.00691 & 0.00035 & 1.805 \\
\hline 9.06734 & 0.00597 & 0.0003 & 1.81347 \\
\hline 9.10984 & 0.00632 & 0.00032 & 1.82197 \\
\hline 9.1525 & 0.00802 & 0.00041 & 1.8305 \\
\hline 9.19531 & 0.00973 & 0.0005 & 1.83906 \\
\hline 9.2375 & 0.00943 & 0.00048 & 1.8475 \\
\hline 9.27984 & 0.00825 & 0.00042 & 1.85597 \\
\hline 9.32172 & 0.00771 & 0.00039 & 1.86434 \\
\hline 9.36328 & 0.00782 & 0.0004 & 1.87266 \\
\hline 9.405 & 0.00904 & 0.00046 & 1.881 \\
\hline 9.4475 & 0.00938 & 0.00048 & 1.8895 \\
\hline 9.49 & 0.00939 & 0.00048 & 1.898 \\
\hline 9.53234 & 0.00899 & 0.00046 & 1.90647 \\
\hline 9.575 & 0.00667 & 0.00034 & 1.915 \\
\hline 9.61781 & 0.0064 & 0.00033 & 1.92356 \\
\hline 9.66031 & 0.00743 & 0.00038 & 1.93206 \\
\hline 9.70281 & 0.00683 & 0.00035 & 1.94056 \\
\hline 9.74562 & 0.00518 & 0.00026 & 1.94912 \\
\hline 9.78859 & 0.00581 & 0.0003 & 1.95772 \\
\hline 9.83094 & 0.00665 & 0.00034 & 1.96619 \\
\hline 9.87297 & 0.00579 & 0.00029 & 1.97459 \\
\hline 9.915 & 0.00683 & 0.00035 & 1.983 \\
\hline 9.95656 & 0.00924 & 0.00047 & 1.99131 \\
\hline 9.99813 & 0.00824 & 0.00042 & 1.99963 \\
\hline 10.04016 & 0.00656 & 0.00033 & 2.00803 \\
\hline 10.08266 & 0.00688 & 0.00035 & 2.01653 \\
\hline 10.12516 & 0.0091 & 0.00046 & 2.02503 \\
\hline 10.1675 & 0.01041 & 0.00053 & 2.0335 \\
\hline 10.21 & 0.01014 & 0.00052 & 2.042 \\
\hline 10.25266 & 0.01184 & 0.0006 & 2.05053 \\
\hline 10.29547 & 0.02322 & 0.00118 & 2.05909 \\
\hline 10.33813 & 0.05366 & 0.00273 & 2.06763 \\
\hline 10.38078 & 0.1094 & 0.00557 & 2.07616 \\
\hline 10.42359 & 0.20549 & 0.01047 & 2.08472 \\
\hline 10.46609 & 0.35293 & 0.01797 & 2.09322 \\
\hline 10.50813 & 0.55303 & 0.02817 & 2.10162 \\
\hline 10.54984 & 0.80156 & 0.04082 & 2.10997 \\
\hline 10.59125 & 1.08824 & 0.05542 & 2.11825 \\
\hline 10.63281 & 1.40841 & 0.07173 & 2.12656 \\
\hline 10.67516 & 1.76402 & 0.08984 & 2.13503 \\
\hline 10.71766 & 2.16002 & 0.11001 & 2.14353 \\
\hline 10.75984 & 2.60252 & 0.13255 & 2.15197 \\
\hline 10.80234 & 3.09444 & 0.1576 & 2.16047 \\
\hline 10.84516 & 3.64382 & 0.18558 & 2.16903 \\
\hline
\end{tabular}




\begin{tabular}{|c|c|c|c|}
\hline $\begin{array}{l}\text { Extension } \\
\text { (mm) }\end{array}$ & $\begin{array}{l}\text { Load } \\
\text { (N) }\end{array}$ & $\begin{array}{c}\text { Compressive stress } \\
\text { (MPa) }\end{array}$ & $\begin{array}{c}\text { Compressive strain } \\
\text { [mm/mm] }\end{array}$ \\
\hline 10.84516 & 3.64382 & 0.18558 & 2.16903 \\
\hline 10.88797 & 4.26099 & 0.21701 & 2.17759 \\
\hline 10.93016 & 4.95567 & 0.25239 & 2.18603 \\
\hline 10.9725 & 5.73307 & 0.29198 & 2.1945 \\
\hline 11.01531 & 6.60011 & 0.33614 & 2.20306 \\
\hline 11.05781 & 7.50258 & 0.3821 & 2.21156 \\
\hline 11.09984 & 8.21063 & 0.41816 & 2.21997 \\
\hline 11.14203 & 8.66602 & 0.44136 & 2.22841 \\
\hline 11.18422 & 8.98211 & 0.45746 & 2.23684 \\
\hline 11.22609 & 9.2991 & 0.4736 & 2.24522 \\
\hline 11.26781 & 9.92897 & 0.50568 & 2.25356 \\
\hline 11.31016 & 10.91099 & 0.55569 & 2.26203 \\
\hline 11.3525 & 12.1878 & 0.62072 & 2.2705 \\
\hline 11.39469 & 13.71009 & 0.69825 & 2.27894 \\
\hline 11.43719 & 15.43442 & 0.78607 & 2.28744 \\
\hline 11.48016 & 17.34896 & 0.88358 & 2.29603 \\
\hline 11.52281 & 19.45444 & 0.99081 & 2.30456 \\
\hline 11.56516 & 21.75485 & 1.10797 & 2.31303 \\
\hline 11.60766 & 24.27113 & 1.23612 & 2.32153 \\
\hline 11.65016 & 27.03672 & 1.37697 & 2.33003 \\
\hline 11.69266 & 30.07842 & 1.53188 & 2.33853 \\
\hline 11.73484 & 33.43922 & 1.70305 & 2.34697 \\
\hline 11.77703 & 37.20161 & 1.89466 & 2.35541 \\
\hline 11.81891 & 41.42402 & 2.10971 & 2.36378 \\
\hline 11.86016 & 46.12899 & 2.34933 & 2.37203 \\
\hline 11.90188 & 51.33019 & 2.61423 & 2.38038 \\
\hline 11.94406 & 57.06573 & 2.90633 & 2.38881 \\
\hline 11.98656 & 63.39763 & 3.22881 & 2.39731 \\
\hline 12.02891 & 70.38905 & 3.58488 & 2.40578 \\
\hline 12.07141 & 78.05219 & 3.97517 & 2.41428 \\
\hline 12.11375 & 86.3261 & 4.39655 & 2.42275 \\
\hline 12.15594 & 95.13129 & 4.845 & 2.43119 \\
\hline 12.19828 & 104.34448 & 5.31422 & 2.43966 \\
\hline 12.24125 & 113.85289 & 5.79848 & 2.44825 \\
\hline 12.28422 & 123.62232 & 6.29603 & 2.45684 \\
\hline 12.32672 & 133.58549 & 6.80345 & 2.46534 \\
\hline 12.36859 & 143.63147 & 7.31509 & 2.47372 \\
\hline 12.41078 & 153.71046 & 7.82841 & 2.48216 \\
\hline 12.45266 & 163.93785 & 8.34929 & 2.49053 \\
\hline 12.49391 & 174.45532 & 8.88494 & 2.49878 \\
\hline 12.53563 & 185.34853 & 9.43972 & 2.50713 \\
\hline 12.57766 & 196.77641 & 10.02174 & 2.51553 \\
\hline 12.61969 & 208.85832 & 10.63707 & 2.52394 \\
\hline 12.66188 & 221.51285 & 11.28156 & 2.53238 \\
\hline 12.70422 & 234.72112 & 11.95425 & 2.54084 \\
\hline 12.74672 & 248.57175 & 12.65965 & 2.54934 \\
\hline 12.78906 & 263.1947 & 13.4044 & 2.55781 \\
\hline 12.83156 & 278.72021 & 14.1951 & 2.56631 \\
\hline 12.87391 & 295.27011 & 15.03798 & 2.57478 \\
\hline 12.91656 & 312.95831 & 15.93884 & 2.58331 \\
\hline 12.95906 & 331.87704 & 16.90236 & 2.59181 \\
\hline 13.00109 & 352.19495 & 17.93714 & 2.60022 \\
\hline 13.04281 & 373.97482 & 19.04638 & 2.60856 \\
\hline 13.08406 & 397.19388 & 20.22892 & 2.61681 \\
\hline 13.12516 & 422.03552 & 21.49409 & 2.62503 \\
\hline 13.16672 & 448.65393 & 22.84976 & 2.63334 \\
\hline 13.17516 & 454.21698 & 23.13308 & 2.63503 \\
\hline
\end{tabular}


Table D.13 Dry Scaffolds Specimen 1 Raw Compression Data

\begin{tabular}{|c|c|c|c|c|c|c|c|}
\hline $\begin{array}{c}\text { Extension } \\
\text { [mm] }\end{array}$ & $\begin{array}{c}\text { Load } \\
\text { (N) }\end{array}$ & $\begin{array}{c}\text { Compressive stress } \\
\text { (MPa) }\end{array}$ & $\begin{array}{c}\text { Compressive strain } \\
\text { [mm/mm] }\end{array}$ & $\begin{array}{c}\text { Extension } \\
\text { (mm) }\end{array}$ & $\begin{array}{l}\text { Load } \\
\text { (N) }\end{array}$ & $\begin{array}{c}\text { Compressive stress } \\
\text { (MPa] }\end{array}$ & $\begin{array}{c}\text { Compressive strain } \\
\text { [mm/mm] }\end{array}$ \\
\hline 0 & -0.00138 & -0.00007 & 0 & 2.71781 & 0.00103 & 0.00005 & 0.54356 \\
\hline 0.00219 & -0.00041 & -0.00002 & 0.00044 & 2.76047 & -0.00108 & -0.00006 & 0.55209 \\
\hline 0.01531 & 0.00873 & 0.00044 & 0.00306 & 2.80312 & -0.00226 & -0.00012 & 0.56062 \\
\hline 0.04875 & 0.03581 & 0.00182 & 0.00975 & 2.84563 & -0.00149 & -0.00008 & 0.56913 \\
\hline 0.09266 & 0.06436 & 0.00328 & 0.01853 & 2.88781 & -0.00174 & -0.00009 & 0.57756 \\
\hline 0.13766 & 0.06823 & 0.00348 & 0.02753 & 2.93016 & -0.00193 & $\begin{array}{l}-0.0001 \\
\end{array}$ & 0.58603 \\
\hline 0.18359 & 0.0512 & 0.00261 & 0.03672 & 2.9725 & -0.00176 & -0.00009 & 0.5945 \\
\hline 0.23281 & 0.03404 & 0.00173 & 0.04656 & 3.01484 & -0.00156 & -0.00008 & 0.60297 \\
\hline 0.28375 & 0.02221 & 0.00113 & 0.05675 & 3.05672 & -0.00193 & -0.0001 & 0.61134 \\
\hline 0.33375 & 0.01077 & 0.00055 & 0.06675 & 3.09813 & -0.00224 & -0.00011 & 0.61963 \\
\hline 0.38266 & 0.00124 & 0.00006 & 0.07653 & 3.13953 & -0.00144 & -0.00007 & 0.62791 \\
\hline 0.43109 & -0.00517 & -0.00026 & 0.08622 & 3.18203 & 0.00202 & 0.0001 & 0.63641 \\
\hline 0.47891 & -0.00605 & -0.00031 & 0.09578 & 3.22469 & 0.00284 & 0.00014 & 0.64494 \\
\hline 0.52609 & -0.00598 & $\begin{array}{l}-0.0003 \\
-0\end{array}$ & 0.10522 & 3.26734 & 0.00078 & 0.00004 & 0.65347 \\
\hline 0.57219 & -0.00678 & -0.00035 & 0.11444 & 3.31 & 0.00174 & 0.00009 & 0.662 \\
\hline 0.61734 & -0.00769 & -0.00039 & 0.12347 & 3.3525 & 0.00276 & 0.00014 & 0.6705 \\
\hline 0.6625 & -0.00632 & -0.00032 & 0.1325 & 3.39469 & 0.00033 & 0.00002 & 0.67894 \\
\hline 0.70797 & -0.00219 & -0.00011 & 0.14159 & 3.43672 & -0.00093 & -0.00005 & 0.68734 \\
\hline 0.75313 & 0.00076 & 0.00004 & 0.15063 & 3.47922 & -0.00111 & -0.00006 & 0.69584 \\
\hline 0.7975 & -0.00074 & -0.00004 & 0.1595 & 3.52219 & -0.00214 & -0.00011 & 0.70444 \\
\hline 0.84188 & -0.00168 & -0.00009 & 0.16838 & 3.565 & -0.00418 & -0.00021 & 0.713 \\
\hline 0.88578 & -0.00138 & -0.00007 & 0.17716 & 3.6075 & -0.00462 & -0.00024 & 0.7215 \\
\hline 0.92953 & -0.00267 & -0.00014 & 0.18591 & 3.64969 & -0.00313 & $\begin{array}{l}-0.00016 \\
\end{array}$ & 0.72994 \\
\hline 0.97344 & -0.00216 & $\begin{array}{l}-0.00011 \\
\end{array}$ & 0.19469 & 3.69156 & -0.00329 & -0.00017 & 0.73831 \\
\hline 1.01766 & -0.00184 & -0.00009 & 0.20353 & 3.73328 & -0.00372 & -0.00019 & 0.74666 \\
\hline 1.06156 & -0.00245 & $\begin{array}{l}-0.00012 \\
\end{array}$ & 0.21231 & 3.77531 & -0.00225 & -0.00011 & 0.75506 \\
\hline 1.10484 & -0.00452 & -0.00023 & 0.22097 & 3.8175 & 0.00193 & 0.0001 & 0.7635 \\
\hline 1.14766 & -0.00456 & -0.00023 & 0.22953 & 3.85969 & 0.00435 & 0.00022 & 0.77194 \\
\hline 1.19016 & -0.00186 & -0.00009 & 0.23803 & 3.90156 & 0.00236 & 0.00012 & 0.78031 \\
\hline 1.23219 & -0.00118 & -0.00006 & 0.24644 & 3.94391 & 0.00064 & 0.00003 & 0.78878 \\
\hline 1.27453 & -0.0024 & -0.00012 & 0.25491 & 3.98656 & 0 & 0 & 0.79731 \\
\hline 1.31734 & -0.00194 & -0.0001 & 0.26347 & 4.02938 & -0.00146 & -0.00007 & 0.80588 \\
\hline 1.36016 & -0.00099 & -0.00005 & 0.27203 & 4.07219 & -0.00121 & -0.00006 & 0.81444 \\
\hline 1.40266 & -0.00009 & 0 & 0.28053 & 4.11516 & 0.00017 & 0.00001 & 0.82303 \\
\hline 1.44547 & 0.00034 & 0.00002 & 0.28909 & 4.15797 & 0.00127 & 0.00006 & 0.83159 \\
\hline 1.48828 & 0.00147 & 0.00007 & 0.29766 & 4.20031 & -0.00025 & -0.00001 & 0.84006 \\
\hline 1.53125 & 0.00209 & 0.00011 & 0.30625 & 4.2425 & -0.00123 & -0.00006 & 0.8485 \\
\hline 1.57406 & 0.00145 & 0.00007 & 0.31481 & 4.285 & -0.00087 & -0.00004 & 0.857 \\
\hline 1.61688 & 0.00089 & 0.00005 & 0.32338 & 4.32688 & -0.00174 & -0.00009 & 0.86538 \\
\hline 1.66 & 0.00171 & 0.00009 & 0.332 & 4.36828 & -0.00247 & $\begin{array}{l}-0.00013 \\
\end{array}$ & 0.87366 \\
\hline 1.70281 & 0.00301 & 0.00015 & 0.34056 & 4.41016 & -0.00273 & -0.00014 & 0.88203 \\
\hline 1.74516 & 0.00198 & 0.0001 & 0.34903 & 4.45281 & -0.00054 & -0.00003 & 0.89056 \\
\hline 1.78719 & -0.00169 & -0.00009 & 0.35744 & 4.49516 & 0.001 & 0.00005 & 0.89903 \\
\hline 1.82906 & -0.00407 & -0.00021 & 0.36581 & 4.53734 & -0.00049 & -0.00003 & 0.90747 \\
\hline 1.87062 & -0.00302 & -0.00015 & 0.37412 & 4.58 & 0.00184 & 0.00009 & 0.916 \\
\hline 1.91266 & -0.00144 & $\begin{array}{l}-0.00007 \\
\end{array}$ & 0.38253 & 4.6225 & 0.00469 & 0.00024 & 0.9245 \\
\hline 1.955 & -0.00046 & -0.00002 & 0.391 & 4.66469 & 0.00291 & 0.00015 & 0.93294 \\
\hline 1.99719 & 0.00175 & 0.00009 & 0.39944 & 4.70719 & -0.00002 & 0 & 0.94144 \\
\hline 2.03969 & 0.0036 & 0.00018 & 0.40794 & 4.75031 & -0.00098 & -0.00005 & 0.95006 \\
\hline 2.08234 & 0.00214 & 0.00011 & 0.41647 & 4.79359 & -0.00015 & -0.00001 & 0.95872 \\
\hline 2.12516 & 0.00094 & 0.00005 & 0.42503 & 4.83625 & -0.00092 & -0.00005 & 0.96725 \\
\hline 2.16781 & 0.00223 & 0.00011 & 0.43356 & 4.87844 & -0.00158 & $\begin{array}{l}-0.00008 \\
\end{array}$ & 0.97569 \\
\hline 2.21031 & 0.0011 & 0.00006 & 0.44206 & 4.92063 & -0.00043 & -0.00002 & 0.98413 \\
\hline 2.25328 & -0.00143 & -0.00007 & 0.45066 & 4.96234 & -0.00012 & -0.00001 & 0.99247 \\
\hline 2.29578 & -0.00282 & -0.00014 & 0.45916 & 5.00375 & -0.00013 & -0.00001 & 1.00075 \\
\hline 2.33781 & -0.00351 & $\begin{array}{l}-0.00018 \\
\end{array}$ & 0.46756 & 5.04547 & 0.00046 & 0.00002 & 1.00909 \\
\hline 2.38016 & -0.00266 & -0.00014 & 0.47603 & 5.08797 & 0.00274 & 0.00014 & 1.01759 \\
\hline 2.42234 & -0.00128 & -0.00007 & 0.48447 & 5.13031 & 0.00312 & 0.00016 & 1.02606 \\
\hline 2.46406 & 0.00078 & 0.00004 & 0.49281 & 5.1725 & -0.00262 & -0.00013 & 1.0345 \\
\hline 2.50563 & 0.00031 & 0.00002 & 0.50113 & 5.21516 & -0.00694 & -0.00035 & 1.04303 \\
\hline 2.54766 & -0.00022 & -0.00001 & 0.50953 & 5.25781 & -0.00429 & -0.00022 & 1.05156 \\
\hline 2.59016 & 0.00118 & 0.00006 & 0.51803 & 5.30031 & 0.00152 & 0.00008 & 1.06006 \\
\hline 2.63266 & 0.00214 & 0.00011 & 0.52653 & 5.34266 & 0.00494 & 0.00025 & 1.06853 \\
\hline 2.67531 & 0.00267 & 0.00014 & 0.53506 & 5.38547 & 0.00416 & 0.00021 & 1.07709 \\
\hline
\end{tabular}




\begin{tabular}{|c|c|c|c|}
\hline $\begin{array}{l}\text { Extension } \\
{[\mathrm{mm}]}\end{array}$ & $\begin{array}{l}\text { Load } \\
\text { (N) }\end{array}$ & $\begin{array}{c}\text { Compressive stress } \\
\text { (MPa) }\end{array}$ & $\begin{array}{c}\text { Compressive strain } \\
{[\mathrm{mm} / \mathrm{mm}]}\end{array}$ \\
\hline 5.42844 & 0.00237 & 0.00012 & 1.08569 \\
\hline 5.47094 & -0.00306 & -0.00016 & 1.09419 \\
\hline 5.51312 & -0.00871 & -0.00044 & 1.10263 \\
\hline 5.55531 & -0.0083 & -0.00042 & 1.11106 \\
\hline 5.59688 & -0.00415 & -0.00021 & 1.11937 \\
\hline 5.63828 & -0.00057 & -0.00003 & 1.12766 \\
\hline 5.68031 & 0.0012 & 0.00006 & 1.13606 \\
\hline 5.72297 & 0.00306 & 0.00016 & 1.14459 \\
\hline 5.76531 & 0.00542 & 0.00028 & 1.15306 \\
\hline 5.8075 & 0.00398 & 0.0002 & 1.1615 \\
\hline 5.85 & 0.00107 & 0.00005 & 1.17 \\
\hline 5.89281 & -0.0018 & -0.00009 & 1.17856 \\
\hline 5.93531 & -0.0042 & -0.00021 & 1.18706 \\
\hline 5.97766 & -0.00556 & -0.00028 & 1.19553 \\
\hline 6.02047 & -0.00281 & -0.00014 & 1.20409 \\
\hline 6.06313 & 0.00234 & 0.00012 & 1.21263 \\
\hline 6.10547 & 0.00257 & 0.00013 & 1.22109 \\
\hline 6.1475 & -0.00031 & -0.00002 & 1.2295 \\
\hline 6.18969 & -0.00139 & -0.00007 & 1.23794 \\
\hline 6.23156 & -0.00076 & -0.00004 & 1.24631 \\
\hline 6.27359 & -0.00071 & -0.00004 & 1.25472 \\
\hline 6.31578 & 0.00029 & 0.00001 & 1.26316 \\
\hline 6.35828 & 0.00073 & 0.00004 & 1.27166 \\
\hline 6.40063 & 0.00023 & 0.00001 & 1.28013 \\
\hline 6.44281 & 0.0005 & 0.00003 & 1.28856 \\
\hline 6.48484 & -0.00103 & -0.00005 & 1.29697 \\
\hline 6.52688 & -0.00242 & -0.00012 & 1.30537 \\
\hline 6.56953 & -0.0038 & -0.00019 & 1.31391 \\
\hline 6.6125 & -0.00245 & -0.00012 & 1.3225 \\
\hline 6.65531 & -0.00139 & -0.00007 & 1.33106 \\
\hline 6.69797 & 0.00098 & 0.00005 & 1.33959 \\
\hline 6.74016 & 0.00466 & 0.00024 & 1.34803 \\
\hline 6.7825 & 0.00668 & 0.00034 & 1.3565 \\
\hline 6.82406 & 0.00367 & 0.00019 & 1.36481 \\
\hline 6.86578 & -0.00112 & -0.00006 & 1.37316 \\
\hline 6.90813 & -0.00335 & -0.00017 & 1.38163 \\
\hline 6.95063 & -0.00465 & -0.00024 & 1.39013 \\
\hline 6.99281 & -0.00217 & -0.00011 & 1.39856 \\
\hline 7.03516 & 0.00156 & 0.00008 & 1.40703 \\
\hline 7.07812 & 0.00331 & 0.00017 & 1.41562 \\
\hline 7.12094 & 0.00315 & 0.00016 & 1.42419 \\
\hline 7.16328 & 0.00114 & 0.00006 & 1.43266 \\
\hline 7.20578 & -0.00101 & -0.00005 & 1.44116 \\
\hline 7.24859 & -0.00074 & -0.00004 & 1.44972 \\
\hline 7.29141 & 0.00039 & 0.00002 & 1.45828 \\
\hline 7.33359 & 0.00112 & 0.00006 & 1.46672 \\
\hline 7.37578 & -0.00169 & -0.00009 & 1.47516 \\
\hline 7.41813 & -0.00189 & -0.0001 & 1.48363 \\
\hline 7.46016 & 0.00065 & 0.00003 & 1.49203 \\
\hline 7.50172 & 0.00375 & 0.00019 & 1.50034 \\
\hline 7.54406 & 0.00759 & 0.00039 & 1.50881 \\
\hline 7.58641 & 0.00782 & 0.0004 & 1.51728 \\
\hline 7.62844 & 0.00477 & 0.00024 & 1.52569 \\
\hline 7.67063 & 0.00298 & 0.00015 & 1.53413 \\
\hline 7.71266 & 0.001 & 0.00005 & 1.54253 \\
\hline 7.75453 & -0.0031 & -0.00016 & 1.55091 \\
\hline 7.79688 & -0.00445 & -0.00023 & 1.55938 \\
\hline 7.84 & -0.00241 & -0.00012 & 1.568 \\
\hline 7.88297 & 0.00042 & 0.00002 & 1.57659 \\
\hline 7.92562 & 0.00119 & 0.00006 & 1.58512 \\
\hline 7.96797 & 0.00096 & 0.00005 & 1.59359 \\
\hline 8.01047 & 0.00248 & 0.00013 & 1.60209 \\
\hline 8.05266 & 0.00355 & 0.00018 & 1.61053 \\
\hline 8.09422 & 0.00236 & 0.00012 & 1.61884 \\
\hline
\end{tabular}

\begin{tabular}{|c|c|c|c|}
\hline $\begin{array}{l}\text { Extension } \\
\text { (mm) }\end{array}$ & $\begin{array}{l}\text { Load } \\
\text { (N) }\end{array}$ & $\begin{array}{c}\text { Compressive stress } \\
\text { (MPa] }\end{array}$ & $\begin{array}{c}\text { Compressive strain } \\
{[\mathrm{mm} / \mathrm{mm}]}\end{array}$ \\
\hline 8.13578 & 0.00097 & 0.00005 & 1.62716 \\
\hline 8.17797 & 0.00252 & 0.00013 & 1.63559 \\
\hline 8.22031 & 0.0029 & 0.00015 & 1.64406 \\
\hline 8.2625 & 0.00175 & 0.00009 & 1.6525 \\
\hline 8.30516 & 0.0022 & 0.00011 & 1.66103 \\
\hline 8.34781 & 0.00175 & 0.00009 & 1.66956 \\
\hline 8.39031 & -0.00049 & -0.00003 & 1.67806 \\
\hline 8.4325 & -0.00256 & -0.00013 & 1.6865 \\
\hline 8.475 & $\begin{array}{l}-0.00119 \\
\end{array}$ & -0.00006 & 1.695 \\
\hline 8.5175 & 0.00042 & 0.00002 & 1.7035 \\
\hline 8.56 & -0.00098 & -0.00005 & 1.712 \\
\hline 8.6025 & -0.00227 & -0.00012 & 1.7205 \\
\hline 8.64484 & -0.00203 & -0.0001 & 1.72897 \\
\hline 8.68688 & -0.00121 & -0.00006 & 1.73738 \\
\hline 8.72859 & -0.00035 & -0.00002 & 1.74572 \\
\hline 8.77031 & 0.0015 & 0.00008 & 1.75406 \\
\hline 8.8125 & 0.00362 & 0.00018 & 1.7625 \\
\hline 8.855 & 0.00426 & 0.00022 & 1.771 \\
\hline 8.89734 & 0.00132 & 0.00007 & 1.77947 \\
\hline 8.94 & -0.0018 & -0.00009 & 1.788 \\
\hline 8.98266 & -0.00325 & -0.00017 & 1.79653 \\
\hline 9.02547 & -0.00205 & -0.0001 & 1.80509 \\
\hline 9.06797 & -0.00253 & -0.00013 & 1.81359 \\
\hline 9.11031 & -0.00392 & -0.0002 & 1.82206 \\
\hline 9.15297 & -0.00148 & -0.00008 & 1.83059 \\
\hline 9.19563 & 0.00035 & 0.00002 & 1.83913 \\
\hline 9.23797 & 0.00094 & 0.00005 & 1.84759 \\
\hline 9.28 & -0.00027 & -0.00001 & 1.856 \\
\hline 9.32172 & -0.00054 & -0.00003 & 1.86434 \\
\hline 9.36328 & -0.0003 & -0.00002 & 1.87266 \\
\hline 9.40516 & 0.00109 & 0.00006 & 1.88103 \\
\hline 9.4475 & 0.00364 & 0.00019 & 1.8895 \\
\hline 9.49 & 0.00402 & 0.0002 & 1.898 \\
\hline 9.5325 & 0.00154 & 0.00008 & 1.9065 \\
\hline 9.57531 & -0.00024 & -0.00001 & 1.91506 \\
\hline 9.61812 & -0.00063 & -0.00003 & 1.92362 \\
\hline 9.66062 & -0.00174 & -0.00009 & 1.93212 \\
\hline 9.70297 & -0.00316 & $\begin{array}{l}-0.00016 \\
\end{array}$ & 1.94059 \\
\hline 9.74578 & -0.00251 & -0.00013 & 1.94916 \\
\hline 9.78875 & -0.0004 & $\begin{array}{c}-0.00002 \\
\end{array}$ & 1.95775 \\
\hline 9.83125 & -0.00143 & -0.00007 & 1.96625 \\
\hline 9.87344 & -0.00377 & -0.00019 & 1.97469 \\
\hline 9.91547 & -0.00326 & -0.00017 & 1.98309 \\
\hline 9.95703 & -0.0001 & 0 & 1.99141 \\
\hline 9.99859 & 0.00135 & 0.00007 & 1.99972 \\
\hline 10.04062 & 0.00129 & 0.00007 & 2.00812 \\
\hline 10.08297 & 0.00118 & 0.00006 & 2.01659 \\
\hline 10.12547 & 0.00197 & 0.0001 & 2.02509 \\
\hline 10.16766 & 0.00186 & 0.00009 & 2.03353 \\
\hline 10.21031 & 0.00016 & 0.00001 & 2.04206 \\
\hline 10.25313 & -0.00026 & -0.00001 & 2.05063 \\
\hline 10.29578 & -0.00006 & 0 & 2.05916 \\
\hline 10.33844 & 0.00063 & 0.00003 & 2.06769 \\
\hline 10.38125 & 0.06104 & 0.00311 & 2.07625 \\
\hline 10.42406 & 0.30455 & 0.01551 & 2.08481 \\
\hline 10.46625 & 0.8193 & 0.04173 & 2.09325 \\
\hline 10.50828 & 1.67287 & 0.0852 & 2.10166 \\
\hline 10.55016 & 2.88188 & 0.14677 & 2.11003 \\
\hline 10.59188 & 4.38977 & 0.22357 & 2.11838 \\
\hline 10.63328 & 5.89882 & 0.30042 & 2.12666 \\
\hline 10.67516 & 7.02508 & 0.35778 & 2.13503 \\
\hline 10.71766 & 7.79742 & 0.39712 & 2.14353 \\
\hline 10.76016 & 8.38463 & 0.42703 & 2.15203 \\
\hline 10.8025 & 9.19912 & 0.46851 & 2.1605 \\
\hline
\end{tabular}




\begin{tabular}{|c|c|c|c|}
\hline $\begin{array}{l}\text { Extension } \\
\text { (mm) }\end{array}$ & $\begin{array}{l}\text { Load } \\
\text { (N) }\end{array}$ & $\begin{array}{c}\text { Compressive stress } \\
\text { (MPa] }\end{array}$ & $\begin{array}{c}\text { Compressive strain } \\
{[\mathrm{mm} / \mathrm{mm}]}\end{array}$ \\
\hline 10.12547 & 0.00197 & 0.0001 & 2.02509 \\
\hline 10.16766 & 0.00186 & 0.00009 & 2.03353 \\
\hline 10.21031 & 0.00016 & 0.00001 & 2.04206 \\
\hline 10.25313 & -0.00026 & -0.00001 & 2.05063 \\
\hline 10.29578 & -0.00006 & 0 & 2.05916 \\
\hline 10.33844 & 0.00063 & 0.00003 & 2.06769 \\
\hline 10.38125 & 0.06104 & 0.00311 & 2.07625 \\
\hline 10.42406 & 0.30455 & 0.01551 & 2.08481 \\
\hline 10.46625 & 0.8193 & 0.04173 & 2.09325 \\
\hline 10.50828 & 1.67287 & 0.0852 & 2.10166 \\
\hline 10.55016 & 2.88188 & 0.14677 & 2.11003 \\
\hline 10.59188 & 4.38977 & 0.22357 & 2.11838 \\
\hline 10.63328 & 5.89882 & 0.30042 & 2.12666 \\
\hline 10.67516 & 7.02508 & 0.35778 & 2.13503 \\
\hline 10.71766 & 7.79742 & 0.39712 & 2.14353 \\
\hline 10.76016 & 8.38463 & 0.42703 & 2.15203 \\
\hline 10.8025 & 9.19912 & 0.46851 & 2.1605 \\
\hline 10.84531 & 10.39145 & 0.52923 & 2.16906 \\
\hline 10.88797 & 11.93097 & 0.60764 & 2.17759 \\
\hline 10.93047 & 13.7487 & 0.70022 & 2.18609 \\
\hline 10.97312 & 15.76809 & 0.80306 & 2.19462 \\
\hline 11.01562 & 17.93523 & 0.91343 & 2.20312 \\
\hline 11.05781 & 20.22678 & 1.03014 & 2.21156 \\
\hline 11.1 & 22.60886 & 1.15146 & 2.22 \\
\hline 11.14219 & 25.07319 & 1.27697 & 2.22844 \\
\hline 11.18406 & 27.62283 & 1.40682 & 2.23681 \\
\hline 11.22578 & 30.27775 & 1.54203 & 2.24516 \\
\hline 11.26766 & 33.07073 & 1.68428 & 2.25353 \\
\hline 11.31016 & 36.07095 & 1.83708 & 2.26203 \\
\hline 11.35266 & 39.33642 & 2.00339 & 2.27053 \\
\hline 11.39469 & 42.87661 & 2.18369 & 2.27894 \\
\hline 11.43719 & 46.66188 & 2.37647 & 2.28744 \\
\hline 11.48 & 50.65111 & 2.57964 & 2.296 \\
\hline 11.52266 & 54.81323 & 2.79161 & 2.30453 \\
\hline 11.56469 & 59.09145 & 3.0095 & 2.31294 \\
\hline 11.60719 & 63.45862 & 3.23192 & 2.32144 \\
\hline 11.64969 & 67.95165 & 3.46075 & 2.32994 \\
\hline 11.69203 & 72.57052 & 3.69599 & 2.33841 \\
\hline 11.73438 & 77.30328 & 3.93702 & 2.34687 \\
\hline 11.77687 & 82.20102 & 4.18646 & 2.35537 \\
\hline 11.81875 & 87.29398 & 4.44585 & 2.36375 \\
\hline 11.86016 & 92.5655 & 4.71432 & 2.37203 \\
\hline 11.90188 & 98.00878 & 4.99155 & 2.38038 \\
\hline 11.94438 & 103.71505 & 5.28216 & 2.38888 \\
\hline 11.98703 & 109.77592 & 5.59084 & 2.39741 \\
\hline 12.02922 & 116.16549 & 5.91626 & 2.40584 \\
\hline 12.07172 & 122.83036 & 6.2557 & 2.41434 \\
\hline 12.11438 & 129.79039 & 6.61017 & 2.42287 \\
\hline 12.15672 & 137.08701 & 6.98178 & 2.43134 \\
\hline 12.19891 & 144.6761 & 7.36829 & 2.43978 \\
\hline 12.24141 & 152.53009 & 7.76829 & 2.44828 \\
\hline 12.28422 & 160.76268 & 8.18758 & 2.45684 \\
\hline 12.32656 & 169.40448 & 8.6277 & 2.46531 \\
\hline 12.36844 & 178.39946 & 9.08581 & 2.47369 \\
\hline 12.41062 & 187.7343 & 9.56123 & 2.48212 \\
\hline 12.45234 & 197.53149 & 10.0602 & 2.49047 \\
\hline 12.49375 & 207.86183 & 10.58632 & 2.49875 \\
\hline 12.53563 & 218.79324 & 11.14305 & 2.50713 \\
\hline 12.57781 & 230.55698 & 11.74217 & 2.51556 \\
\hline 12.62 & 243.3231 & 12.39234 & 2.524 \\
\hline 12.66219 & 257.06174 & 13.09205 & 2.53244 \\
\hline 12.70438 & 271.78055 & 13.84167 & 2.54087 \\
\hline 12.74672 & 287.48749 & 14.64162 & 2.54934 \\
\hline 12.78891 & 304.29517 & 15.49763 & 2.55778 \\
\hline 12.83125 & 322.25668 & 16.4124 & 2.56625 \\
\hline 12.87375 & 341.49084 & 17.39198 & 2.57475 \\
\hline 12.91609 & 362.17172 & 18.44525 & 2.58322 \\
\hline 12.95828 & 384.35797 & 19.57519 & 2.59166 \\
\hline 13.00016 & 408.07974 & 20.78333 & 2.60003 \\
\hline 13.04203 & 433.314 & 22.0685 & 2.60841 \\
\hline 13.07484 & 454.61847 & 23.15353 & 2.61497 \\
\hline 13.12516 & 422.03552 & 21.49409 & 2.62503 \\
\hline 13.16672 & 448.65393 & 22.84976 & 2.63334 \\
\hline 13.17516 & 454.21698 & 23.13308 & 2.63503 \\
\hline
\end{tabular}


Table D.14 Dry Scaffolds Specimen 2 Raw Compression Data

\begin{tabular}{|c|c|c|c|c|c|c|c|}
\hline $\begin{array}{c}\text { Extension } \\
\text { (mm) }\end{array}$ & $\begin{array}{l}\text { Load } \\
\text { [N] }\end{array}$ & $\begin{array}{c}\text { Compressive stress } \\
\text { (MPa] }\end{array}$ & $\begin{array}{c}\text { Compressive strain } \\
\text { [mmimm] }\end{array}$ & $\begin{array}{c}\begin{array}{c}\text { Extension } \\
(\mathrm{mm})\end{array} \\
\end{array}$ & $\begin{array}{c}\text { Load } \\
\text { (N) }\end{array}$ & \begin{tabular}{|c|} 
Compressive stress \\
(MPa)
\end{tabular} & $\begin{array}{c}\text { Compressive strain } \\
\text { [mmimm] }\end{array}$ \\
\hline 0 & 0.0004 & 0.00002 & $\mid 0$ & 2.71766 & 0.00104 & 0.00005 & 0.54353 \\
\hline 0.00266 & 0.00132 & 0.00007 & 0.00053 & 2.76016 & 0.00335 & 0.00017 & 0.55203 \\
\hline 0.02266 & 0.0166 & 0.00085 & 0.00453 & 2.80281 & 0.00323 & 0.00016 & 0.56056 \\
\hline 0.06406 & 0.05415 & 0.00276 & 0.01281 & 2.84531 & 0.00135 & 0.00007 & 0.56906 \\
\hline 0.11 & 0.07811 & 0.00398 & 0.022 & 2.8875 & 0.00126 & 0.00006 & 0.5775 \\
\hline 0.15156 & 0.06313 & 0.00322 & 0.03031 & 2.92969 & 0.00255 & 0.00013 & 0.58594 \\
\hline 0.19422 & 0.03427 & 0.00175 & 0.03884 & 2.97203 & 0.0027 & 0.00014 & 0.59441 \\
\hline 0.24266 & 0.02214 & 0.00113 & 0.04853 & 3.01469 & 0.00017 & 0.00001 & 0.60294 \\
\hline 0.29328 & 0.02069 & 0.00105 & 0.05866 & 3.05656 & -0.00094 & -0.00005 & 0.61131 \\
\hline 0.34203 & 0.01211 & 0.00062 & 0.06841 & 3.09828 & -0.00008 & 0 & 0.61966 \\
\hline 0.38938 & -0.00012 & -0.00001 & 0.07788 & 3.14031 & 0.00056 & 0.00003 & 0.62806 \\
\hline 0.43656 & -0.00484 & -0.00025 & 0.08731 & 3.18297 & 0.00236 & 0.00012 & 0.63659 \\
\hline 0.48422 & -0.00178 & -0.00009 & 0.09684 & 3.22547 & 0.00381 & 0.00019 & 0.64509 \\
\hline 0.53125 & -0.00178 & -0.00009 & 0.10625 & 3.26766 & 0.0026 & 0.00013 & 0.65353 \\
\hline 0.57641 & -0.00632 & -0.00032 & 0.11528 & 3.31 & 0.00064 & 0.00003 & 0.662 \\
\hline 0.62047 & -0.00697 & -0.00035 & 0.12409 & 3.3525 & 0.00076 & 0.00004 & 0.6705 \\
\hline 0.66516 & -0.00261 & -0.00013 & 0.13303 & 3.39453 & 0.00216 & 0.00011 & 0.67891 \\
\hline 0.71031 & 0.0001 & 0 & 0.14206 & 3.43672 & 0.00306 & 0.00016 & 0.68734 \\
\hline 0.75516 & 0.002 & 0.0001 & 0.15103 & 3.47953 & 0.00168 & 0.00009 & 0.69591 \\
\hline 0.79922 & 0.00103 & 0.00005 & 0.15984 & 3.52234 & 0.00083 & 0.00004 & 0.70447 \\
\hline 0.84312 & -0.0008 & -0.00004 & 0.16862 & 3.56516 & -0.00099 & -0.00005 & 0.71303 \\
\hline 0.88672 & -0.00274 & -0.00014 & 0.17734 & 3.60766 & -0.00277 & -0.00014 & 0.72153 \\
\hline 0.93031 & -0.00283 & -0.00014 & 0.18606 & 3.64984 & -0.00446 & -0.00023 & 0.72997 \\
\hline 0.97422 & -0.00082 & -0.00004 & 0.19484 & 3.69156 & -0.00363 & -0.00018 & 0.73831 \\
\hline 1.01844 & 0.00032 & 0.00002 & 0.20369 & 3.73312 & -0.00228 & -0.00012 & 0.74663 \\
\hline 1.06203 & -0.00034 & -0.00002 & 0.21241 & 3.77484 & -0.00105 & -0.00005 & 0.75497 \\
\hline 1.10516 & -0.00199 & -0.0001 & 0.22103 & 3.81687 & 0.00197 & 0.0001 & 0.76337 \\
\hline 1.14813 & -0.00152 & -0.00008 & 0.22963 & 3.85938 & 0.00492 & 0.00025 & 0.77188 \\
\hline 1.19063 & 0.00004 & 0 & 0.23813 & 3.90219 & 0.00738 & 0.00038 & 0.78044 \\
\hline 1.23281 & 0.00013 & 0.00001 & 0.24656 & 3.945 & 0.00704 & 0.00036 & 0.789 \\
\hline 1.27531 & -0.00048 & -0.00002 & 0.25506 & 3.98734 & 0.00387 & 0.0002 & 0.79747 \\
\hline 1.31813 & -0.00169 & -0.00009 & 0.26362 & 4.02953 & 0.00245 & 0.00012 & 0.80591 \\
\hline 1.36094 & -0.00115 & -0.00006 & 0.27219 & 4.07219 & 0.00293 & 0.00015 & 0.81444 \\
\hline 1.40344 & -0.00057 & -0.00003 & 0.28069 & 4.11484 & 0.00326 & 0.00017 & 0.82297 \\
\hline 1.44594 & -0.00081 & -0.00004 & 0.28919 & 4.15734 & 0.00247 & 0.00013 & 0.83147 \\
\hline 1.48875 & -0.00035 & -0.00002 & 0.29775 & 4.2 & 0.00211 & 0.00011 & 0.84 \\
\hline 1.53156 & -0.00072 & -0.00004 & 0.30631 & 4.2425 & 0.00223 & 0.00011 & 0.8485 \\
\hline 1.57422 & -0.00194 & -0.0001 & 0.31484 & 4.285 & -0.00071 & -0.00004 & 0.857 \\
\hline 1.61703 & -0.00011 & -0.00001 & 0.32341 & 4.32688 & -0.00249 & -0.00013 & 0.86538 \\
\hline 1.65984 & 0.00303 & 0.00015 & 0.33197 & 4.36828 & -0.00018 & -0.00001 & 0.87366 \\
\hline 1.7025 & 0.00338 & 0.00017 & 0.3405 & 4.41016 & 0.00156 & 0.00008 & 0.88203 \\
\hline 1.74469 & 0.00289 & 0.00015 & 0.34894 & 4.45234 & 0.00179 & 0.00009 & 0.89047 \\
\hline 1.78703 & 0.00236 & 0.00012 & 0.35741 & 4.49438 & 0.00158 & 0.00008 & 0.89888 \\
\hline 1.82906 & 0.00183 & 0.00009 & 0.36581 & 4.53688 & 0.0032 & 0.00016 & 0.90738 \\
\hline 1.87078 & -0.00076 & -0.00004 & 0.37416 & 4.57969 & 0.00552 & 0.00028 & 0.91594 \\
\hline 1.91266 & -0.00015 & -0.00001 & 0.38253 & 4.62281 & 0.00597 & 0.0003 & 0.92456 \\
\hline 1.955 & 0.00239 & 0.00012 & 0.391 & 4.66563 & 0.00618 & 0.00031 & 0.93313 \\
\hline 1.9975 & 0.00362 & 0.00018 & 0.3995 & 4.70844 & 0.00346 & 0.00018 & 0.94169 \\
\hline 2.03969 & 0.00148 & 0.00008 & 0.40794 & 4.75125 & 0.00122 & 0.00006 & 0.95025 \\
\hline 2.08203 & -0.00018 & -0.00001 & 0.41641 & 4.79391 & 0.00055 & 0.00003 & 0.95878 \\
\hline 2.12469 & 0.00134 & 0.00007 & 0.42494 & 4.83625 & 0.00003 & 0 & 0.96725 \\
\hline 2.1675 & 0.00229 & 0.00012 & 0.4335 & 4.87844 & 0.00085 & 0.00004 & 0.97569 \\
\hline 2.21031 & 0.00221 & 0.00011 & 0.44206 & 4.92063 & 0.00099 & 0.00005 & 0.98413 \\
\hline 2.25328 & 0.00301 & 0.00015 & 0.45066 & 4.96219 & -0.00006 & 0 & 0.99244 \\
\hline 2.29594 & 0.00281 & 0.00014 & 0.45919 & 5.00359 & -0.00069 & -0.00004 & 1.00072 \\
\hline 2.33844 & 0.00046 & 0.00002 & 0.46769 & 5.04547 & 0.00031 & 0.00002 & 1.00909 \\
\hline 2.38078 & -0.00088 & -0.00004 & 0.47616 & 5.08797 & 0.00303 & 0.00015 & 1.01759 \\
\hline 2.42281 & -0.00084 & -0.00004 & 0.48456 & 5.13063 & 0.00371 & 0.00019 & 1.02613 \\
\hline 2.46438 & -0.00031 & -0.00002 & 0.49288 & 5.17297 & 0.00326 & 0.00017 & 1.03459 \\
\hline 2.50609 & -0.00037 & -0.00002 & 0.50122 & 5.21516 & 0.00252 & 0.00013 & 1.04303 \\
\hline 2.54797 & -0.00022 & -0.00001 & 0.50959 & 5.25766 & 0.00029 & 0.00002 & 1.05153 \\
\hline 2.59047 & 0.00053 & 0.00003 & 0.51809 & 5.30031 & 0.00044 & 0.00002 & 1.06006 \\
\hline 2.63281 & 0.00126 & 0.00006 & 0.52656 & 5.34297 & 0.00162 & 0.00008 & 1.06859 \\
\hline 2.67516 & 0.0017 & 0.00009 & 0.53503 & 5.38609 & 0.0023 & 0.00012 & 1.07722 \\
\hline
\end{tabular}




\begin{tabular}{|c|c|c|c|c|c|c|c|}
\hline $\begin{array}{c}\text { Extension } \\
(\mathrm{mm})\end{array}$ & $\begin{array}{l}\text { Load } \\
\text { (N) }\end{array}$ & $\begin{array}{c}\text { Compressive stress } \\
\text { [MPa] }\end{array}$ & $\begin{array}{c}\text { Compressive strain } \\
\text { [mmlmm }]\end{array}$ & $\begin{array}{c}\text { Extension } \\
(\mathbf{m m})\end{array}$ & $\begin{array}{c}\text { Load } \\
\text { (N) }\end{array}$ & $\begin{array}{c}\text { Compressive stress } \\
\text { (MPa) }\end{array}$ & $\begin{array}{c}\text { Compressive strain } \\
{[\mathrm{mm} / \mathrm{mm})}\end{array}$ \\
\hline 5.42891 & 0.00268 & 0.00014 & 1.08578 & 8.13547 & 0.00182 & 0.00009 & 1.62709 \\
\hline 5.47125 & 0.00046 & 0.00002 & 1.09425 & 8.17766 & 0.00084 & 0.00004 & 1.63553 \\
\hline 5.51344 & -0.00003 & 0 & 1.10269 & 8.22031 & 0.00217 & 0.00011 & 1.64406 \\
\hline 5.55531 & -0.00063 & -0.00003 & 1.11106 & 8.26266 & 0.00427 & 0.00022 & 1.65253 \\
\hline 5.59688 & -0.002 & -0.0001 & 1.11937 & 8.305 & 0.00381 & 0.00019 & 1.661 \\
\hline 5.63828 & -0.00057 & -0.00003 & 1.12766 & 8.3475 & 0.00322 & 0.00016 & 1.6695 \\
\hline 5.68031 & 0.00176 & 0.00009 & 1.13606 & 8.39 & 0.0028 & 0.00014 & 1.678 \\
\hline 5.72281 & 0.00205 & 0.0001 & 1.14456 & 8.43234 & 0.00273 & 0.00014 & 1.68647 \\
\hline 5.76516 & 0.00105 & 0.00005 & 1.15303 & 8.47453 & 0.0036 & 0.00018 & 1.69491 \\
\hline 5.8075 & 0.00115 & 0.00006 & 1.1615 & 8.51734 & 0.00441 & 0.00022 & 1.70347 \\
\hline 5.85 & 0.00246 & 0.00013 & 1.17 & 8.56016 & 0.00467 & 0.00024 & 1.71203 \\
\hline 5.89266 & 0.00304 & 0.00015 & 1.17853 & 8.6025 & 0.00368 & 0.00019 & 1.7205 \\
\hline 5.93531 & 0.00235 & 0.00012 & 1.18706 & 8.64484 & 0.00169 & 0.00009 & 1.72897 \\
\hline 5.97813 & 0.00167 & 0.00009 & 1.19563 & 8.68719 & -0.0003 & -0.00002 & 1.73744 \\
\hline 6.02063 & 0.00199 & 0.0001 & 1.20413 & 8.72891 & -0.00041 & -0.00002 & 1.74578 \\
\hline 6.06297 & 0.00289 & 0.00015 & 1.21259 & 8.77063 & 0.00026 & 0.00001 & 1.75412 \\
\hline 6.10547 & 0.00317 & 0.00016 & 1.22109 & 8.81281 & 0.00136 & 0.00007 & 1.76256 \\
\hline 6.14781 & 0.003 & 0.00015 & 1.22956 & 8.85516 & 0.00159 & 0.00008 & 1.77103 \\
\hline 6.19016 & 0.00176 & 0.00009 & 1.23803 & 8.8975 & 0.00256 & 0.00013 & 1.7795 \\
\hline 6.23172 & 0.00071 & 0.00004 & 1.24634 & 8.93984 & 0.00413 & 0.00021 & 1.78797 \\
\hline 6.27359 & 0.00058 & 0.00003 & 1.25472 & 8.98266 & 0.00379 & 0.00019 & 1.79653 \\
\hline 6.31578 & 0.00082 & 0.00004 & 1.26316 & 9.02547 & 0.00411 & 0.00021 & 1.80509 \\
\hline 6.35813 & 0.00312 & 0.00016 & 1.27163 & 9.06781 & 0.0041 & 0.00021 & 1.81356 \\
\hline 6.40047 & 0.0037 & 0.00019 & 1.28009 & 9.11016 & 0.00338 & 0.00017 & 1.82203 \\
\hline 6.44266 & 0.00258 & 0.00013 & 1.28853 & 9.15297 & 0.00307 & 0.00016 & 1.83059 \\
\hline 6.48453 & 0.00301 & 0.00015 & 1.29691 & 9.19547 & 0.00332 & 0.00017 & 1.83909 \\
\hline 6.52672 & 0.00334 & 0.00017 & 1.30534 & 9.23766 & 0.00622 & 0.00032 & 1.84753 \\
\hline 6.56938 & 0.00449 & 0.00023 & 1.31388 & 9.28 & 0.00595 & 0.0003 & 1.856 \\
\hline 6.6125 & 0.00508 & 0.00026 & 1.3225 & 9.32219 & 0.00355 & 0.00018 & 1.86444 \\
\hline 6.65562 & 0.0046 & 0.00023 & 1.33113 & 9.36375 & 0.00197 & 0.0001 & 1.87275 \\
\hline 6.69812 & 0.00221 & 0.00011 & 1.33963 & 9.40563 & 0.00247 & 0.00013 & 1.88113 \\
\hline 6.74047 & -0.00088 & -0.00004 & 1.34809 & 9.44781 & 0.0037 & 0.00019 & 1.88956 \\
\hline 6.78281 & 0.00075 & 0.00004 & 1.35656 & 9.49031 & 0.00386 & 0.0002 & 1.89806 \\
\hline 6.82469 & 0.00261 & 0.00013 & 1.36494 & 9.53266 & 0.00415 & 0.00021 & 1.90653 \\
\hline 6.86625 & 0.00087 & 0.00004 & 1.37325 & 9.57531 & 0.00435 & 0.00022 & 1.91506 \\
\hline 6.90813 & 0.00039 & 0.00002 & 1.38163 & 9.61797 & 0.00326 & 0.00017 & 1.92359 \\
\hline 6.95078 & 0.00331 & 0.00017 & 1.39016 & 9.66047 & 0.00341 & 0.00017 & 1.93209 \\
\hline 6.99312 & 0.00655 & 0.00033 & 1.39863 & 9.70297 & 0.00456 & 0.00023 & 1.94059 \\
\hline 7.03531 & 0.00484 & 0.00025 & 1.40706 & 9.74578 & 0.00583 & 0.0003 & 1.94916 \\
\hline 7.07797 & 0.00291 & 0.00015 & 1.41559 & 9.78875 & 0.00489 & 0.00025 & 1.95775 \\
\hline 7.12078 & 0.00435 & 0.00022 & 1.42416 & 9.83125 & 0.00199 & 0.0001 & 1.96625 \\
\hline 7.16344 & 0.00366 & 0.00019 & 1.43269 & 9.87328 & 0.00068 & 0.00003 & 1.97466 \\
\hline 7.20609 & 0.0021 & 0.00011 & 1.44122 & 9.91531 & 0.00115 & 0.00006 & 1.98306 \\
\hline 7.24875 & 0.00164 & 0.00008 & 1.44975 & 9.95703 & 0.00209 & 0.00011 & 1.99141 \\
\hline 7.29125 & 0.00199 & 0.0001 & 1.45825 & 9.99859 & 0.00254 & 0.00013 & 1.99972 \\
\hline 7.33359 & 0.00204 & 0.0001 & 1.46672 & 10.04047 & 0.00421 & 0.00021 & 2.00809 \\
\hline 7.37578 & -0.00015 & -0.00001 & 1.47516 & 10.08313 & 0.00631 & 0.00032 & 2.01662 \\
\hline 7.41797 & -0.00143 & -0.00007 & 1.48359 & 10.12562 & 0.00567 & 0.00029 & 2.02513 \\
\hline 7.45984 & -0.00116 & -0.00006 & 1.49197 & 10.16797 & 0.00381 & 0.00019 & 2.03359 \\
\hline 7.50141 & -0.00014 & -0.00001 & 1.50028 & 10.21047 & 0.0056 & 0.00028 & 2.04209 \\
\hline 7.54359 & 0.00232 & 0.00012 & 1.50872 & 10.25328 & 0.00618 & 0.00031 & 2.05066 \\
\hline 7.58594 & 0.00502 & 0.00026 & 1.51719 & 10.29594 & 0.0038 & 0.00019 & 2.05919 \\
\hline 7.62813 & 0.00609 & 0.00031 & 1.52562 & 10.33875 & 0.00158 & 0.00008 & 2.06775 \\
\hline 7.67063 & 0.00526 & 0.00027 & 1.53413 & 10.38141 & 0.00171 & 0.00009 & 2.07628 \\
\hline 7.71313 & 0.00441 & 0.00022 & 1.54263 & 10.42391 & 0.00225 & 0.00011 & 2.08478 \\
\hline 7.75547 & 0.00374 & 0.00019 & 1.55109 & 10.46641 & 0.00193 & 0.0001 & 2.09328 \\
\hline 7.7975 & 0.00268 & 0.00014 & 1.5595 & 10.50859 & 0.00406 & 0.00021 & 2.10172 \\
\hline 7.84 & 0.0011 & 0.00006 & 1.568 & 10.55031 & 0.00418 & 0.00021 & 2.11006 \\
\hline 7.88312 & 0.00049 & 0.00002 & 1.57662 & 10.59188 & 0.00185 & 0.00009 & 2.11838 \\
\hline 7.92578 & 0.00078 & 0.00004 & 1.58516 & 10.63328 & 0.00243 & 0.00012 & 2.12666 \\
\hline 7.96797 & 0.00098 & 0.00005 & 1.59359 & 10.67531 & 0.00519 & 0.00026 & 2.13506 \\
\hline 8.01031 & 0.00063 & 0.00003 & 1.60206 & 10.71781 & 0.03464 & 0.00176 & 2.14356 \\
\hline 8.0525 & 0.00142 & 0.00007 & 1.6105 & 10.76016 & 0.22603 & 0.01151 & 2.15203 \\
\hline 8.09406 & 0.00306 & 0.00016 & 1.61881 & 10.80281 & 0.71468 & 0.0364 & 2.16056 \\
\hline
\end{tabular}




\begin{tabular}{|c|c|c|c|}
\hline $\begin{array}{l}\text { Extension } \\
\text { [mm] }\end{array}$ & $\begin{array}{l}\text { Load } \\
\text { [N] }\end{array}$ & $\begin{array}{c}\text { Compressive stress } \\
\text { (MPa] }\end{array}$ & $\begin{array}{c}\text { Compressive strain } \\
{[\mathrm{mm} / \mathrm{mm}]}\end{array}$ \\
\hline 10.84562 & 1.58653 & 0.0808 & 2.16913 \\
\hline 10.88844 & 2.80112 & 0.14266 & 2.17769 \\
\hline 10.93062 & 4.31485 & 0.21975 & 2.18613 \\
\hline 10.97297 & 5.96397 & 0.30374 & 2.19459 \\
\hline 11.01578 & 7.27882 & 0.37071 & 2.20316 \\
\hline 11.05828 & 8.18394 & 0.4168 & 2.21166 \\
\hline 11.10031 & 8.79383 & 0.44787 & 2.22006 \\
\hline 11.14234 & 9.47725 & 0.48267 & 2.22847 \\
\hline 11.18437 & 10.59932 & 0.53982 & 2.23688 \\
\hline 11.22609 & 12.17302 & 0.61997 & 2.24522 \\
\hline 11.26797 & 14.09433 & 0.71782 & 2.25359 \\
\hline 11.31047 & 16.23116 & 0.82665 & 2.26209 \\
\hline 11.35297 & 18.47513 & 0.94093 & 2.27059 \\
\hline 11.39516 & 20.80408 & 1.05954 & 2.27903 \\
\hline 11.4375 & 23.22751 & 1.18297 & 2.2875 \\
\hline 11.48016 & 25.77348 & 1.31263 & 2.29603 \\
\hline 11.52266 & 28.46136 & 1.44953 & 2.30453 \\
\hline 11.565 & 31.30739 & 1.59447 & 2.313 \\
\hline 11.60766 & 34.34226 & 1.74904 & 2.32153 \\
\hline 11.65047 & 37.58517 & 1.9142 & 2.33009 \\
\hline 11.69297 & 41.02451 & 2.08936 & 2.33859 \\
\hline 11.73516 & 44.62528 & 2.27275 & 2.34703 \\
\hline 11.77734 & 48.43493 & 2.46677 & 2.35547 \\
\hline 11.81938 & 52.53782 & 2.67573 & 2.36387 \\
\hline 11.86063 & 56.93398 & 2.89962 & 2.37213 \\
\hline 11.90234 & 61.56123 & 3.13529 & 2.38047 \\
\hline 11.94453 & 66.41525 & 3.3825 & 2.38891 \\
\hline 11.98719 & 71.57847 & 3.64546 & 2.39744 \\
\hline 12.02953 & 77.1149 & 3.92743 & 2.40591 \\
\hline 12.07219 & 83.08215 & 4.23134 & 2.41444 \\
\hline 12.115 & 89.50249 & 4.55832 & 2.423 \\
\hline 12.15719 & 96.44513 & 4.91191 & 2.43144 \\
\hline 12.19922 & 103.88535 & 5.29084 & 2.43984 \\
\hline 12.24187 & 111.83197 & 5.69556 & 2.44837 \\
\hline 12.28453 & 120.37619 & 6.13071 & 2.45691 \\
\hline 12.32687 & 129.47144 & 6.59393 & 2.46537 \\
\hline 12.36906 & 138.98889 & 7.07865 & 2.47381 \\
\hline 12.41125 & 148.95959 & 7.58645 & 2.48225 \\
\hline 12.45297 & 159.50981 & 8.12377 & 2.49059 \\
\hline 12.49422 & 170.70563 & 8.69397 & 2.49884 \\
\hline 12.53563 & 182.56931 & 9.29818 & 2.50713 \\
\hline 12.57781 & 195.23376 & 9.94317 & 2.51556 \\
\hline 12.62 & 208.89072 & 10.63872 & 2.524 \\
\hline 12.66172 & 223.50333 & 11.38293 & 2.53234 \\
\hline 12.70391 & 239.0289 & 12.17364 & 2.54078 \\
\hline 12.74625 & 255.61121 & 13.01817 & 2.54925 \\
\hline 12.78875 & 273.38184 & 13.92322 & 2.55775 \\
\hline 12.83125 & 292.37604 & 14.89059 & 2.56625 \\
\hline 12.87359 & 312.61771 & 15.92149 & 2.57472 \\
\hline 12.91609 & 334.18463 & 17.01988 & 2.58322 \\
\hline 12.95813 & 357.14807 & 18.1894 & 2.59162 \\
\hline 12.99984 & 381.58044 & 19.43373 & 2.59997 \\
\hline 13.04141 & 407.45776 & 20.75165 & 2.60828 \\
\hline 13.08281 & 434.80969 & 22.14468 & 2.61656 \\
\hline 13.1075 & 452.0434 & 23.02238 & 2.6215 \\
\hline 13.16672 & 448.65393 & 22.84976 & 2.63334 \\
\hline 13.17516 & 454.21698 & 23.13308 & 2.63503 \\
\hline
\end{tabular}


Table D.15 Dry Scaffolds Specimen 3 Raw Compression Data

\begin{tabular}{|c|c|c|c|}
\hline $\begin{array}{l}\text { Extension } \\
\text { (mm) }\end{array}$ & $\begin{array}{c}\text { Load } \\
\text { (N) }\end{array}$ & \begin{tabular}{|c|} 
Compressive stress \\
(MPa)
\end{tabular} & $\begin{array}{c}\text { Compressive strain } \\
\text { [mm/mm] }\end{array}$ \\
\hline 0 & 0.00349 & 0.00018 & 0 \\
\hline 0.00281 & 0.0049 & 0.00025 & 0.00056 \\
\hline 0.02391 & 0.02062 & 0.00105 & 0.00478 \\
\hline 0.06578 & 0.05735 & 0.00292 & 0.01316 \\
\hline 0.11172 & 0.08143 & 0.00415 & 0.02234 \\
\hline 0.15328 & 0.06731 & 0.00343 & 0.03066 \\
\hline 0.19625 & 0.03766 & 0.00192 & 0.03925 \\
\hline 0.24484 & 0.0231 & 0.00118 & 0.04897 \\
\hline 0.29516 & 0.02079 & 0.00106 & 0.05903 \\
\hline 0.34375 & 0.01458 & 0.00074 & 0.06875 \\
\hline 0.39078 & 0.00419 & 0.00021 & 0.07816 \\
\hline 0.43797 & -0.00128 & -0.00006 & 0.08759 \\
\hline 0.485 & -0.00041 & -0.00002 & 0.097 \\
\hline 0.53156 & -0.00032 & -0.00002 & 0.10631 \\
\hline 0.57688 & -0.00225 & -0.00011 & 0.11538 \\
\hline 0.62141 & -0.00294 & -0.00015 & 0.12428 \\
\hline 0.66609 & 0.00017 & 0.00001 & 0.13322 \\
\hline 0.71109 & 0.00411 & 0.00021 & 0.14222 \\
\hline 0.75578 & 0.00441 & 0.00022 & 0.15116 \\
\hline 0.79984 & 0.00135 & 0.00007 & 0.15997 \\
\hline 0.84359 & 0.00258 & 0.00013 & 0.16872 \\
\hline 0.88719 & 0.00492 & 0.00025 & 0.17744 \\
\hline 0.93062 & 0.00305 & 0.00016 & 0.18612 \\
\hline 0.97438 & 0.00252 & 0.00013 & 0.19488 \\
\hline 1.01844 & 0.00309 & 0.00016 & 0.20369 \\
\hline 1.06203 & 0.00211 & 0.00011 & 0.21241 \\
\hline 1.10516 & 0.00011 & 0.00001 & 0.22103 \\
\hline 1.14812 & 0.00035 & 0.00002 & 0.22962 \\
\hline 1.19062 & 0.00307 & 0.00016 & 0.23812 \\
\hline 1.23281 & 0.00311 & 0.00016 & 0.24656 \\
\hline 1.27484 & 0.00158 & 0.00008 & 0.25497 \\
\hline 1.31766 & 0.00316 & 0.00016 & 0.26353 \\
\hline 1.36031 & 0.00618 & 0.00031 & 0.27206 \\
\hline 1.40266 & 0.00728 & 0.00037 & 0.28053 \\
\hline 1.44547 & 0.00717 & 0.00037 & 0.28909 \\
\hline 1.48828 & 0.00736 & 0.00037 & 0.29766 \\
\hline 1.53109 & 0.00521 & 0.00027 & 0.30622 \\
\hline 1.57375 & 0.00173 & 0.00009 & 0.31475 \\
\hline 1.61656 & 0.00143 & 0.00007 & 0.32331 \\
\hline 1.65953 & 0.0035 & 0.00018 & 0.33191 \\
\hline 1.70234 & 0.00528 & 0.00027 & 0.34047 \\
\hline 1.74453 & 0.00513 & 0.00026 & 0.34891 \\
\hline 1.78672 & 0.00468 & 0.00024 & 0.35734 \\
\hline 1.82891 & 0.00507 & 0.00026 & 0.36578 \\
\hline 1.87063 & 0.00504 & 0.00026 & 0.37413 \\
\hline 1.91234 & 0.00381 & 0.00019 & 0.38247 \\
\hline 1.95453 & 0.0037 & 0.00019 & 0.39091 \\
\hline 1.99703 & 0.00529 & 0.00027 & 0.39941 \\
\hline 2.03922 & 0.0055 & 0.00028 & 0.40784 \\
\hline 2.08188 & 0.00354 & 0.00018 & 0.41638 \\
\hline 2.12469 & 0.00507 & 0.00026 & 0.42494 \\
\hline 2.1675 & 0.00723 & 0.00037 & 0.4335 \\
\hline 2.21016 & 0.00624 & 0.00032 & 0.44203 \\
\hline 2.25297 & 0.00312 & 0.00016 & 0.45059 \\
\hline 2.29562 & 0.00184 & 0.00009 & 0.45913 \\
\hline 2.33781 & 0.00364 & 0.00019 & 0.46756 \\
\hline 2.38016 & 0.00431 & 0.00022 & 0.47603 \\
\hline 2.42234 & 0.004 & 0.0002 & 0.48447 \\
\hline 2.46406 & 0.00496 & 0.00025 & 0.49281 \\
\hline 2.50547 & 0.00515 & 0.00026 & 0.50109 \\
\hline 2.54734 & 0.00502 & 0.00026 & 0.50947 \\
\hline 2.58969 & 0.00527 & 0.00027 & 0.51794 \\
\hline 2.63219 & 0.00674 & 0.00034 & 0.52644 \\
\hline 2.67469 & 0.00744 & 0.00038 & 0.53494 \\
\hline
\end{tabular}

\begin{tabular}{|c|c|c|c|}
\hline $\begin{array}{l}\text { Extension } \\
\text { (mm) }\end{array}$ & $\begin{array}{l}\text { Load } \\
\text { (N) }\end{array}$ & $\begin{array}{c}\text { Compressive stress } \\
\text { (MPa) }\end{array}$ & $\begin{array}{c}\text { Compressive strain } \\
\text { (mm/mm) }\end{array}$ \\
\hline 2.71766 & 0.00611 & 0.00031 & 0.54353 \\
\hline 2.76047 & 0.00502 & 0.00026 & 0.55209 \\
\hline 2.80297 & 0.0036 & 0.00018 & 0.56059 \\
\hline 2.84516 & 0.00305 & 0.00016 & 0.56903 \\
\hline 2.8875 & 0.00393 & 0.0002 & 0.5775 \\
\hline 2.93031 & 0.00483 & 0.00025 & 0.58606 \\
\hline 2.97266 & 0.00548 & 0.00028 & 0.59453 \\
\hline 3.01484 & 0.00381 & 0.00019 & 0.60297 \\
\hline 3.05672 & 0.00333 & 0.00017 & 0.61134 \\
\hline 3.09813 & 0.00394 & 0.0002 & 0.61963 \\
\hline 3.13984 & 0.0039 & 0.0002 & 0.62797 \\
\hline 3.18219 & 0.00492 & 0.00025 & 0.63644 \\
\hline 3.22469 & 0.00441 & 0.00022 & 0.64494 \\
\hline 3.26703 & 0.00434 & 0.00022 & 0.65341 \\
\hline 3.30938 & 0.0058 & 0.0003 & 0.66188 \\
\hline 3.35203 & 0.00612 & 0.00031 & 0.67041 \\
\hline 3.39406 & 0.0045 & 0.00023 & 0.67881 \\
\hline 3.43578 & 0.00359 & 0.00018 & 0.68716 \\
\hline 3.47828 & 0.00469 & 0.00024 & 0.69566 \\
\hline 3.52156 & 0.00591 & 0.0003 & 0.70431 \\
\hline 3.56422 & 0.00566 & 0.00029 & 0.71284 \\
\hline 3.60656 & 0.00612 & 0.00031 & 0.72131 \\
\hline 3.64906 & 0.00677 & 0.00034 & 0.72981 \\
\hline 3.69141 & 0.00578 & 0.00029 & 0.73828 \\
\hline 3.73344 & 0.00411 & 0.00021 & 0.74669 \\
\hline 3.77531 & 0.00392 & 0.0002 & 0.75506 \\
\hline 3.81766 & 0.00468 & 0.00024 & 0.76353 \\
\hline 3.86 & 0.00454 & 0.00023 & 0.772 \\
\hline 3.90219 & 0.00608 & 0.00031 & 0.78044 \\
\hline 3.94469 & 0.0061 & 0.00031 & 0.78894 \\
\hline 3.98719 & 0.00495 & 0.00025 & 0.79744 \\
\hline 4.02969 & 0.00468 & 0.00024 & 0.80594 \\
\hline 4.07203 & 0.00489 & 0.00025 & 0.81441 \\
\hline 4.11453 & 0.00721 & 0.00037 & 0.82291 \\
\hline 4.15734 & 0.00804 & 0.00041 & 0.83147 \\
\hline 4.19984 & 0.007 & 0.00036 & 0.83997 \\
\hline 4.24219 & 0.00682 & 0.00035 & 0.84844 \\
\hline 4.28453 & 0.00673 & 0.00034 & 0.85691 \\
\hline 4.32625 & 0.00625 & 0.00032 & 0.86525 \\
\hline 4.3675 & 0.00504 & 0.00026 & 0.8735 \\
\hline 4.40938 & 0.00421 & 0.00021 & 0.88188 \\
\hline 4.45219 & 0.00606 & 0.00031 & 0.89044 \\
\hline 4.495 & 0.00734 & 0.00037 & 0.899 \\
\hline 4.53734 & 0.00673 & 0.00034 & 0.90747 \\
\hline 4.58 & 0.00625 & 0.00032 & 0.916 \\
\hline 4.62281 & 0.00617 & 0.00031 & 0.92456 \\
\hline 4.66531 & 0.00496 & 0.00025 & 0.93306 \\
\hline 4.70766 & 0.00396 & 0.0002 & 0.94153 \\
\hline 4.75062 & 0.00674 & 0.00034 & 0.95012 \\
\hline 4.79359 & 0.00876 & 0.00045 & 0.95872 \\
\hline 4.83578 & 0.00744 & 0.00038 & 0.96716 \\
\hline 4.87797 & 0.00809 & 0.00041 & 0.97559 \\
\hline 4.91984 & 0.00845 & 0.00043 & 0.98397 \\
\hline 4.96156 & 0.00523 & 0.00027 & 0.99231 \\
\hline 5.00313 & 0.00344 & 0.00018 & 1.00063 \\
\hline 5.04516 & 0.0056 & 0.00029 & 1.00903 \\
\hline 5.08734 & 0.00773 & 0.00039 & 1.01747 \\
\hline 5.12953 & 0.00703 & 0.00036 & 1.02591 \\
\hline 5.17203 & 0.00648 & 0.00033 & 1.03441 \\
\hline 5.215 & 0.00757 & 0.00039 & 1.043 \\
\hline 5.25828 & 0.00921 & 0.00047 & 1.05166 \\
\hline 5.30094 & 0.00848 & 0.00043 & 1.06019 \\
\hline 5.34312 & 0.00676 & 0.00034 & 1.06862 \\
\hline 5.38594 & 0.00589 & 0.0003 & 1.07719 \\
\hline
\end{tabular}




\begin{tabular}{|c|c|c|c|}
\hline $\begin{array}{l}\text { Extension } \\
\text { (mm) }\end{array}$ & $\begin{array}{l}\text { Load } \\
\text { (N) }\end{array}$ & $\begin{array}{c}\text { Compressive stress } \\
\text { (MPa] }\end{array}$ & $\begin{array}{c}\text { Compressive strain } \\
{[\mathrm{mm} / \mathrm{mm}]}\end{array}$ \\
\hline 5.42875 & 0.00626 & 0.00032 & 1.08575 \\
\hline 5.47109 & 0.0042 & 0.00021 & 1.09422 \\
\hline 5.51328 & 0.00287 & 0.00015 & 1.10266 \\
\hline 5.555 & 0.0034 & 0.00017 & 1.111 \\
\hline 5.59625 & 0.00529 & 0.00027 & 1.11925 \\
\hline 5.63766 & 0.00556 & 0.00028 & 1.12753 \\
\hline 5.67969 & 0.00585 & 0.0003 & 1.13594 \\
\hline 5.72219 & 0.00738 & 0.00038 & 1.14444 \\
\hline 5.76453 & 0.00749 & 0.00038 & 1.15291 \\
\hline 5.80703 & 0.00687 & 0.00035 & 1.16141 \\
\hline 5.84953 & 0.00727 & 0.00037 & 1.16991 \\
\hline 5.89203 & 0.0081 & 0.00041 & 1.17841 \\
\hline 5.93453 & 0.0058 & 0.0003 & 1.18691 \\
\hline 5.97766 & 0.0032 & 0.00016 & 1.19553 \\
\hline 6.02062 & 0.00497 & 0.00025 & 1.20412 \\
\hline 6.06313 & 0.00706 & 0.00036 & 1.21263 \\
\hline 6.10531 & 0.00546 & 0.00028 & 1.22106 \\
\hline 6.1475 & 0.00254 & 0.00013 & 1.2295 \\
\hline 6.18953 & 0.00266 & 0.00014 & 1.23791 \\
\hline 6.23141 & 0.0034 & 0.00017 & 1.24628 \\
\hline 6.27344 & 0.00259 & 0.00013 & 1.25469 \\
\hline 6.31578 & 0.00305 & 0.00016 & 1.26316 \\
\hline 6.35797 & 0.00482 & 0.00025 & 1.27159 \\
\hline 6.40016 & 0.00687 & 0.00035 & 1.28003 \\
\hline 6.44266 & 0.00793 & 0.0004 & 1.28853 \\
\hline 6.48438 & 0.00759 & 0.00039 & 1.29688 \\
\hline 6.52625 & 0.00676 & 0.00034 & 1.30525 \\
\hline 6.56906 & 0.00777 & 0.0004 & 1.31381 \\
\hline 6.61203 & 0.00741 & 0.00038 & 1.32241 \\
\hline 6.655 & 0.00695 & 0.00035 & 1.331 \\
\hline 6.6975 & 0.00706 & 0.00036 & 1.3395 \\
\hline 6.74031 & 0.00753 & 0.00038 & 1.34806 \\
\hline 6.78281 & 0.00689 & 0.00035 & 1.35656 \\
\hline 6.82469 & 0.00622 & 0.00032 & 1.36494 \\
\hline 6.86609 & 0.00678 & 0.00035 & 1.37322 \\
\hline 6.90797 & 0.00761 & 0.00039 & 1.38159 \\
\hline 6.95047 & 0.00824 & 0.00042 & 1.39009 \\
\hline 6.99266 & 0.00928 & 0.00047 & 1.39853 \\
\hline 7.03484 & 0.01081 & 0.00055 & 1.40697 \\
\hline 7.0775 & 0.00879 & 0.00045 & 1.4155 \\
\hline 7.12047 & 0.00634 & 0.00032 & 1.42409 \\
\hline 7.16297 & 0.00603 & 0.00031 & 1.43259 \\
\hline 7.20547 & 0.00623 & 0.00032 & 1.44109 \\
\hline 7.24828 & 0.00663 & 0.00034 & 1.44966 \\
\hline 7.29109 & 0.00686 & 0.00035 & 1.45822 \\
\hline 7.33328 & 0.00788 & 0.0004 & 1.46666 \\
\hline 7.37547 & 0.00535 & 0.00027 & 1.47509 \\
\hline 7.41781 & 0.00333 & 0.00017 & 1.48356 \\
\hline 7.45953 & 0.00494 & 0.00025 & 1.49191 \\
\hline 7.50094 & 0.00574 & 0.00029 & 1.50019 \\
\hline 7.54297 & 0.00706 & 0.00036 & 1.50859 \\
\hline 7.58578 & 0.00866 & 0.00044 & 1.51716 \\
\hline 7.62813 & 0.00833 & 0.00042 & 1.52562 \\
\hline 7.67047 & 0.00728 & 0.00037 & 1.53409 \\
\hline 7.7125 & 0.0068 & 0.00035 & 1.5425 \\
\hline 7.75484 & 0.00701 & 0.00036 & 1.55097 \\
\hline 7.79719 & 0.00723 & 0.00037 & 1.55944 \\
\hline 7.84 & 0.00703 & 0.00036 & 1.568 \\
\hline 7.88281 & 0.0073 & 0.00037 & 1.57656 \\
\hline 7.92547 & 0.00696 & 0.00035 & 1.58509 \\
\hline 7.96797 & 0.00736 & 0.00037 & 1.59359 \\
\hline 8.01047 & 0.00862 & 0.00044 & 1.60209 \\
\hline 8.05234 & 0.00685 & 0.00035 & 1.61047 \\
\hline 8.09375 & 0.00463 & 0.00024 & 1.61875 \\
\hline
\end{tabular}

\begin{tabular}{|c|c|c|c|}
\hline $\begin{array}{c}\text { Extension } \\
\text { (mm) }\end{array}$ & $\begin{array}{l}\text { Load } \\
\text { (N) }\end{array}$ & $\begin{array}{c}\text { Compressive stress } \\
\text { (MPa] }\end{array}$ & $\begin{array}{c}\text { Compressive strain } \\
\text { (mm/mm) }\end{array}$ \\
\hline 8.13516 & 0.00427 & 0.00022 & 1.62703 \\
\hline 8.17734 & 0.00762 & 0.00039 & 1.63547 \\
\hline 8.21984 & 0.00971 & 0.00049 & 1.64397 \\
\hline 8.26203 & 0.00889 & 0.00045 & 1.65241 \\
\hline 8.30438 & 0.00871 & 0.00044 & 1.66088 \\
\hline 8.34719 & 0.00859 & 0.00044 & 1.66944 \\
\hline 8.38984 & 0.00748 & 0.00038 & 1.67797 \\
\hline 8.43203 & 0.006 & 0.00031 & 1.68641 \\
\hline 8.47437 & 0.00697 & 0.00036 & 1.69488 \\
\hline 8.51703 & 0.00731 & 0.00037 & 1.70341 \\
\hline 8.55969 & 0.00607 & 0.00031 & 1.71194 \\
\hline 8.60203 & 0.00638 & 0.00033 & 1.72041 \\
\hline 8.64437 & 0.00668 & 0.00034 & 1.72888 \\
\hline 8.68656 & 0.00596 & 0.0003 & 1.73731 \\
\hline 8.72828 & 0.00563 & 0.00029 & 1.74566 \\
\hline 8.77016 & 0.00683 & 0.00035 & 1.75403 \\
\hline 8.81234 & 0.00834 & 0.00042 & 1.76247 \\
\hline 8.85484 & 0.00874 & 0.00044 & 1.77097 \\
\hline 8.89734 & 0.00877 & 0.00045 & 1.77947 \\
\hline 8.93984 & 0.01017 & 0.00052 & 1.78797 \\
\hline 8.98234 & 0.0107 & 0.00054 & 1.79647 \\
\hline 9.025 & 0.00961 & 0.00049 & 1.805 \\
\hline 9.06719 & 0.00703 & 0.00036 & 1.81344 \\
\hline 9.10953 & 0.00748 & 0.00038 & 1.82191 \\
\hline 9.1525 & 0.00895 & 0.00046 & 1.8305 \\
\hline 9.19531 & 0.00776 & 0.0004 & 1.83906 \\
\hline 9.23766 & 0.00701 & 0.00036 & 1.84753 \\
\hline 9.27984 & 0.00701 & 0.00036 & 1.85597 \\
\hline 9.32187 & 0.00675 & 0.00034 & 1.86437 \\
\hline 9.36344 & 0.00542 & 0.00028 & 1.87269 \\
\hline 9.40531 & 0.00652 & 0.00033 & 1.88106 \\
\hline 9.4475 & 0.01003 & 0.00051 & 1.8895 \\
\hline 9.48984 & 0.01086 & 0.00055 & 1.89797 \\
\hline 9.53219 & 0.00827 & 0.00042 & 1.90644 \\
\hline 9.57484 & 0.00646 & 0.00033 & 1.91497 \\
\hline 9.61781 & 0.00936 & 0.00048 & 1.92356 \\
\hline 9.66047 & 0.01094 & 0.00056 & 1.93209 \\
\hline 9.70266 & 0.00951 & 0.00048 & 1.94053 \\
\hline 9.74547 & 0.00873 & 0.00044 & 1.94909 \\
\hline 9.78844 & 0.00915 & 0.00047 & 1.95769 \\
\hline 9.83078 & 0.0074 & 0.00038 & 1.96616 \\
\hline 9.87281 & 0.00534 & 0.00027 & 1.97456 \\
\hline 9.91484 & 0.00694 & 0.00035 & 1.98297 \\
\hline 9.95656 & 0.00834 & 0.00042 & 1.99131 \\
\hline 9.99813 & 0.00813 & 0.00041 & 1.99963 \\
\hline 10.04016 & 0.00914 & 0.00047 & 2.00803 \\
\hline 10.08266 & 0.01075 & 0.00055 & 2.01653 \\
\hline 10.12516 & 0.01265 & 0.00064 & 2.02503 \\
\hline 10.16766 & 0.04938 & 0.00251 & 2.03353 \\
\hline 10.21031 & 0.19726 & 0.01005 & 2.04206 \\
\hline 10.25297 & 0.49889 & 0.02541 & 2.05059 \\
\hline 10.29547 & 0.95366 & 0.04857 & 2.05909 \\
\hline 10.33813 & 1.52765 & 0.0778 & 2.06763 \\
\hline 10.38078 & 2.19395 & 0.11174 & 2.07616 \\
\hline 10.42359 & 2.90843 & 0.14813 & 2.08472 \\
\hline 10.46594 & 3.66994 & 0.18691 & 2.09319 \\
\hline 10.50813 & 4.51327 & 0.22986 & 2.10162 \\
\hline 10.54984 & 5.51541 & 0.2809 & 2.10997 \\
\hline 10.59141 & 6.69626 & 0.34104 & 2.11828 \\
\hline 10.63328 & 7.88149 & 0.4014 & 2.12666 \\
\hline 10.67562 & 8.84217 & 0.45033 & 2.13512 \\
\hline 10.71797 & 9.57255 & 0.48753 & 2.14359 \\
\hline 10.75984 & 10.12072 & 0.51544 & 2.15197 \\
\hline 10.80219 & 10.69228 & 0.54455 & 2.16044 \\
\hline
\end{tabular}




\begin{tabular}{|c|c|c|c|}
\hline $\begin{array}{c}\text { Extension } \\
\text { [mm] }\end{array}$ & $\begin{array}{l}\text { Load } \\
\text { (N) }\end{array}$ & $\begin{array}{c}\text { Compressive stress } \\
\text { [MPa] }\end{array}$ & $\begin{array}{c}\text { Compressive strain } \\
{[\mathrm{mm} / \mathrm{mm}]}\end{array}$ \\
\hline 10.84531 & 11.56652 & 0.58908 & 2.16906 \\
\hline 10.88781 & 12.7691 & 0.65032 & 2.17756 \\
\hline 10.93 & 14.21677 & 0.72405 & 2.186 \\
\hline 10.9725 & 15.81088 & 0.80524 & 2.1945 \\
\hline 11.01531 & 17.4944 & 0.89098 & 2.20306 \\
\hline 11.05766 & 19.25548 & 0.98067 & 2.21153 \\
\hline 11.09969 & 21.09995 & 1.07461 & 2.21994 \\
\hline 11.14172 & 23.03618 & 1.17322 & 2.22834 \\
\hline 11.18375 & 25.0706 & 1.27683 & 2.23675 \\
\hline 11.22547 & 27.20315 & 1.38545 & 2.24509 \\
\hline 11.26734 & 29.43281 & 1.499 & 2.25347 \\
\hline 11.30969 & 31.77008 & 1.61804 & 2.26194 \\
\hline 11.35203 & 34.23186 & 1.74341 & 2.27041 \\
\hline 11.39422 & 36.83514 & 1.876 & 2.27884 \\
\hline 11.43688 & 39.60259 & 2.01694 & 2.28738 \\
\hline 11.47984 & 42.55877 & 2.1675 & 2.29597 \\
\hline 11.5225 & 45.72979 & 2.329 & 2.3045 \\
\hline 11.56469 & 49.10007 & 2.50065 & 2.31294 \\
\hline 11.60734 & 52.65464 & 2.68168 & 2.32147 \\
\hline 11.65016 & 56.42234 & 2.87357 & 2.33003 \\
\hline 11.69281 & 60.41324 & 3.07682 & 2.33856 \\
\hline 11.735 & 64.61469 & 3.2908 & 2.347 \\
\hline 11.77688 & 69.02387 & 3.51536 & 2.35538 \\
\hline 11.81859 & 73.63483 & 3.75019 & 2.36372 \\
\hline 11.86 & 78.4874 & 3.99733 & 2.372 \\
\hline 11.90156 & 83.58681 & 4.25704 & 2.38031 \\
\hline 11.94391 & 88.96931 & 4.53117 & 2.38878 \\
\hline 11.98641 & 94.69003 & 4.82252 & 2.39728 \\
\hline 12.02859 & 100.73645 & 5.13047 & 2.40572 \\
\hline 12.07109 & 107.10078 & 5.4546 & 2.41422 \\
\hline 12.11391 & 113.80087 & 5.79583 & 2.42278 \\
\hline 12.15672 & 120.91165 & 6.15798 & 2.43134 \\
\hline 12.19906 & 128.44466 & 6.54163 & 2.43981 \\
\hline 12.24172 & 136.35413 & 6.94446 & 2.44834 \\
\hline 12.28453 & 144.69849 & 7.36943 & 2.45691 \\
\hline 12.32672 & 153.49159 & 7.81726 & 2.46534 \\
\hline 12.36859 & 162.66373 & 8.2844 & 2.47372 \\
\hline 12.41094 & 172.26712 & 8.77349 & 2.48219 \\
\hline 12.4525 & 182.45827 & 9.29252 & 2.4905 \\
\hline 12.49359 & 193.29327 & 9.84435 & 2.49872 \\
\hline 12.53516 & 204.78305 & 10.42951 & 2.50703 \\
\hline 12.57734 & 217.11099 & 11.05737 & 2.51547 \\
\hline 12.61938 & 230.43979 & 11.7362 & 2.52387 \\
\hline 12.66156 & 244.71819 & 12.46339 & 2.53231 \\
\hline 12.70391 & 259.93341 & 13.2383 & 2.54078 \\
\hline 12.74641 & 276.22092 & 14.06782 & 2.54928 \\
\hline 12.78891 & 293.73895 & 14.96 & 2.55778 \\
\hline 12.83109 & 312.55463 & 15.91828 & 2.56622 \\
\hline 12.87313 & 332.65372 & 16.94191 & 2.57463 \\
\hline 12.91563 & 354.07156 & 18.03272 & 2.58313 \\
\hline 12.95781 & 376.92587 & 19.19668 & 2.59156 \\
\hline 12.99984 & 401.33389 & 20.43977 & 2.59997 \\
\hline 13.04188 & 427.34116 & 21.76431 & 2.60838 \\
\hline 13.08297 & 455.00189 & 23.17306 & 2.61659 \\
\hline
\end{tabular}


Table D.16 Dry Scaffolds Specimen 4 Raw Compression Data

\begin{tabular}{|c|c|c|c|c|c|c|c|}
\hline $\begin{array}{c}\text { Extension } \\
\text { (mm) }\end{array}$ & $\begin{array}{l}\text { Load } \\
\text { (N) }\end{array}$ & \begin{tabular}{|c|} 
Compressive stress \\
(MPa)
\end{tabular} & $\begin{array}{c}\text { Compressive strain } \\
\text { [mm/mm] }\end{array}$ & $\begin{array}{c}\text { Extension } \\
\text { (mm) }\end{array}$ & $\begin{array}{l}\text { Load } \\
\text { (N) }\end{array}$ & $\begin{array}{c}\text { Compressive stress } \\
\text { (MPa] }\end{array}$ & $\begin{array}{c}\text { Compressive strain } \\
\text { [mmimm] }\end{array}$ \\
\hline 0 & 0.00114 & \begin{tabular}{|c|}
0.00006 \\
\end{tabular} & 0 & 2.71766 & 0.00631 & 0.00032 & 0.54353 \\
\hline 0.00281 & 0.00339 & 0.00017 & 0.00056 & 2.76031 & 0.00391 & 0.0002 & 0.55206 \\
\hline 0.02375 & 0.02039 & 0.00104 & 0.00475 & 2.80266 & 0.00448 & 0.00023 & 0.56053 \\
\hline 0.06578 & 0.05596 & 0.00285 & 0.01316 & 2.84516 & 0.00434 & 0.00022 & 0.56903 \\
\hline 0.11172 & 0.07851 & 0.004 & 0.02234 & 2.88766 & 0.00207 & 0.00011 & 0.57753 \\
\hline 0.15313 & 0.0633 & 0.00322 & 0.03063 & 2.93 & 0.00407 & 0.00021 & 0.586 \\
\hline 0.19609 & 0.03469 & 0.00177 & 0.03922 & 2.97234 & 0.00512 & 0.00026 & 0.59447 \\
\hline 0.24484 & 0.02388 & 0.00122 & 0.04897 & 3.01453 & 0.00085 & 0.00004 & 0.60291 \\
\hline 0.29531 & 0.02417 & 0.00123 & 0.05906 & 3.05641 & -0.000039 & -0.00002 & 0.61128 \\
\hline 0.34375 & 0.01474 & 0.00075 & 0.06875 & 3.09797 & 0.00249 & 0.00013 & 0.61959 \\
\hline 0.39094 & 0.00337 & 0.00017 & 0.07819 & 3.13969 & 0.00351 & 0.00018 & 0.62794 \\
\hline 0.43797 & -0.00201 & -0.0001 & 0.08759 & 3.18234 & 0.00356 & 0.00018 & 0.63647 \\
\hline 0.48516 & -0.002 & $\begin{array}{c}-0.0001 \\
\end{array}$ & 0.09703 & 3.22469 & 0.00525 & 0.00027 & 0.64494 \\
\hline 0.53172 & -0.00231 & -0.00012 & 0.10634 & $\begin{array}{l}3.26+05 \\
3.26688 \\
\end{array}$ & 0.0065 & 0.00033 & 0.65338 \\
\hline 0.57688 & -0.0054 & -0.00028 & 0.11538 & 3.30922 & 0.00288 & 0.00015 & 0.66184 \\
\hline 0.62141 & -0.00417 & -0.00021 & 0.12428 & 3.35187 & 0.00227 & 0.00012 & 0.67037 \\
\hline 0.66625 & -0.00057 & -0.00003 & 0.13325 & 3.39422 & 0.00556 & 0.00028 & 0.67884 \\
\hline 0.71125 & 0.00023 & 0.00001 & 0.14225 & 3.43609 & 0.00562 & 0.00029 & 0.68722 \\
\hline 0.75563 & 0.00216 & 0.00011 & 0.15112 & 3.47859 & 0.00311 & 0.00016 & 0.69572 \\
\hline 0.79969 & 0.00272 & 0.00014 & 0.15994 & 3.52141 & 0.0025 & 0.00013 & 0.70428 \\
\hline 0.84375 & 0.00374 & 0.00019 & 0.16875 & 3.56437 & 0.00425 & 0.00022 & 0.71288 \\
\hline 0.88734 & 0.00356 & 0.00018 & 0.17747 & 3.60734 & 0.00768 & $\begin{array}{l}0.000<2 \\
0.00024\end{array}$ & 0.72147 \\
\hline 0.93078 & 0.00172 & 0.00009 & 0.18616 & 3.64969 & 0.00314 & 0.00016 & 0.72994 \\
\hline 0.97453 & 0.00178 & 0.00009 & 0.19491 & 3.69172 & 0.00375 & 0.00019 & 0.73834 \\
\hline 1.01859 & 0.00244 & 0.00012 & 0.20372 & 3.73328 & 0.0023 & 0.00012 & 0.74666 \\
\hline 1.06234 & -0.00015 & -0.00001 & 0.21247 & 3.77531 & 0.00069 & 0.00004 & 0.75506 \\
\hline 1.10531 & -0.00192 & -0.0001 & 0.22106 & 3.8175 & 0.00381 & 0.00019 & 0.7635 \\
\hline 1.14797 & -0.00244 & -0.00012 & 0.22959 & 3.85984 & 0.00675 & 0.00034 & 0.77197 \\
\hline 1.19047 & -0.0017 & -0.00009 & 0.23809 & 3.90203 & 0.00498 & 0.00025 & 0.78041 \\
\hline 1.23266 & -0.00091 & -0.00005 & 0.24653 & 3.94438 & 0.00465 & 0.00024 & 0.78888 \\
\hline 1.275 & 0.0012 & 0.00006 & 0.255 & 3.98688 & 0.00458 & 0.00023 & 0.79738 \\
\hline 1.31766 & 0.00328 & 0.00017 & 0.26353 & 4.02906 & 0.00182 & 0.00009 & 0.80581 \\
\hline 1.36031 & 0.00473 & 0.00024 & 0.27206 & 4.07156 & 0.00259 & 0.00013 & 0.81431 \\
\hline 1.40266 & 0.00541 & 0.00028 & 0.28053 & 4.11453 & 0.00695 & 0.00035 & 0.82291 \\
\hline 1.44547 & 0.00681 & 0.00035 & 0.28909 & 4.1575 & 0.0076 & 0.00039 & 0.8315 \\
\hline 1.48844 & 0.00824 & 0.00042 & 0.29769 & 4.19969 & 0.00473 & 0.00024 & 0.83994 \\
\hline 1.53109 & 0.00601 & 0.00031 & 0.30622 & 4.24156 & 0.00303 & 0.00015 & 0.84831 \\
\hline 1.57375 & 0.00375 & 0.00019 & 0.31475 & 4.28359 & 0.00177 & 0.00009 & 0.85672 \\
\hline 1.61656 & 0.00271 & 0.00014 & 0.32331 & 4.32594 & 0.00282 & 0.00014 & 0.86519 \\
\hline 1.65906 & 0.00192 & 0.0001 & 0.33181 & 4.36812 & 0.00517 & 0.00026 & 0.87363 \\
\hline 1.70203 & 0.00316 & 0.00016 & 0.34041 & 4.41031 & 0.00612 & 0.00031 & 0.88206 \\
\hline 1.74453 & 0.00292 & 0.00015 & 0.34891 & 4.45266 & 0.00524 & 0.00027 & 0.89053 \\
\hline 1.78672 & 0.00197 & 0.0001 & 0.35734 & 4.495 & 0.00497 & 0.00025 & 0.899 \\
\hline 1.82844 & -0.00047 & -0.00002 & 0.36569 & 4.53734 & 0.00482 & 0.00025 & 0.90747 \\
\hline 1.87016 & $\begin{array}{l}-0.00165 \\
\end{array}$ & -0.00008 & 0.37403 & 4.58031 & 0.00497 & 0.00025 & 0.91606 \\
\hline 1.91234 & 0.00146 & 0.00007 & 0.38247 & 4.62313 & 0.00469 & 0.00024 & 0.92463 \\
\hline 1.95469 & 0.00579 & 0.00029 & 0.39094 & 4.66531 & 0.00443 & 0.00023 & 0.93306 \\
\hline 1.99703 & 0.00695 & 0.00035 & 0.39941 & 4.70766 & 0.00436 & 0.00022 & 0.94153 \\
\hline 2.03953 & 0.00562 & 0.00029 & 0.40791 & 4.75031 & 0.00314 & 0.00016 & 0.95006 \\
\hline 2.08234 & 0.00447 & 0.00023 & 0.41647 & 4.79328 & 0.00249 & 0.00013 & 0.95866 \\
\hline 2.12531 & 0.00456 & 0.00023 & 0.42506 & 4.83594 & 0.00463 & 0.00024 & 0.96719 \\
\hline 2.16766 & 0.00349 & 0.00018 & 0.43353 & 4.87828 & 0.00461 & 0.00023 & 0.97566 \\
\hline 2.21016 & 0.00161 & 0.00008 & 0.44203 & 4.91969 & 0.00255 & 0.00013 & 0.98394 \\
\hline 2.25328 & 0.0033 & 0.00017 & 0.45066 & 4.96078 & 0.00017 & 0.00001 & 0.99216 \\
\hline 2.29594 & 0.00363 & 0.00018 & 0.45919 & 5.0025 & 0.00089 & 0.00005 & 1.0005 \\
\hline 2.33813 & 0.00139 & 0.00007 & 0.46763 & 5.04531 & 0.00425 & 0.00022 & 1.00906 \\
\hline 2.38016 & -0.00018 & -0.00001 & 0.47603 & 5.08828 & 0.00616 & 0.00031 & 1.01766 \\
\hline 2.42219 & 0.00185 & 0.00009 & 0.48444 & 5.13047 & 0.00484 & 0.00025 & 1.02609 \\
\hline 2.46375 & 0.00229 & 0.00012 & 0.49275 & 5.1725 & 0.00454 & 0.00023 & 1.0345 \\
\hline 2.505 & 0.0006 & 0.00003 & 0.501 & 5.215 & 0.004 & 0.0002 & 1.043 \\
\hline 2.54719 & 0.00078 & 0.00004 & 0.50944 & 5.25766 & 0.00304 & 0.00015 & 1.05153 \\
\hline 2.58984 & 0.00231 & 0.00012 & 0.51797 & 5.30047 & 0.00341 & 0.00017 & 1.06009 \\
\hline 2.63234 & 0.00485 & 0.00025 & 0.52647 & 5.34312 & 0.00427 & 0.00022 & 1.06862 \\
\hline 2.675 & 0.00499 & 0.00025 & 0.535 & 5.38594 & 0.00482 & 0.00025 & 1.07719 \\
\hline
\end{tabular}




\begin{tabular}{|c|c|c|c|}
\hline $\begin{array}{c}\text { Extension } \\
\text { (mm) }\end{array}$ & $\begin{array}{l}\text { Load } \\
\text { (N) }\end{array}$ & $\begin{array}{c}\text { Compressive stress } \\
\text { [MPa] }\end{array}$ & $\begin{array}{c}\text { Compressive strain } \\
\text { [mm/mm })\end{array}$ \\
\hline 5.42859 & 0.005 & 0.00025 & 1.08572 \\
\hline 5.47109 & 0.00273 & 0.00014 & 1.09422 \\
\hline 5.51328 & 0.00304 & 0.00016 & 1.10266 \\
\hline 5.55516 & 0.00384 & 0.0002 & 1.11103 \\
\hline 5.59641 & 0.0033 & 0.00017 & 1.11928 \\
\hline 5.63734 & 0.00397 & 0.0002 & 1.12747 \\
\hline 5.67938 & 0.00681 & 0.00035 & 1.13588 \\
\hline 5.72203 & 0.00726 & 0.00037 & 1.14441 \\
\hline 5.76438 & 0.00624 & 0.00032 & 1.15288 \\
\hline 5.80703 & 0.0052 & 0.00026 & 1.16141 \\
\hline 5.85 & 0.00692 & 0.00035 & 1.17 \\
\hline 5.89281 & 0.00543 & 0.00028 & 1.17856 \\
\hline 5.93516 & 0.00199 & 0.0001 & 1.18703 \\
\hline 5.97766 & 0.00551 & 0.00028 & 1.19553 \\
\hline 6.02047 & 0.00514 & 0.00026 & 1.20409 \\
\hline 6.06297 & 0.00362 & 0.00018 & 1.21259 \\
\hline 6.10531 & 0.00585 & 0.0003 & 1.22106 \\
\hline 6.14766 & 0.00552 & 0.00028 & 1.22953 \\
\hline 6.18969 & 0.00167 & 0.00009 & 1.23794 \\
\hline 6.23141 & 0.00178 & 0.00009 & 1.24628 \\
\hline 6.27328 & 0.00459 & 0.00023 & 1.25466 \\
\hline 6.31562 & 0.00544 & 0.00028 & 1.26312 \\
\hline 6.35766 & 0.00486 & 0.00025 & 1.27153 \\
\hline 6.39984 & 0.00587 & 0.0003 & 1.27997 \\
\hline 6.44219 & 0.00491 & 0.00025 & 1.28844 \\
\hline 6.48422 & 0.00215 & 0.00011 & 1.29684 \\
\hline 6.52641 & 0.00458 & 0.00023 & 1.30528 \\
\hline 6.56922 & 0.00759 & 0.00039 & 1.31384 \\
\hline 6.6125 & 0.00706 & 0.00036 & 1.3225 \\
\hline 6.65578 & 0.00675 & 0.00034 & 1.33116 \\
\hline 6.69828 & 0.00524 & 0.00027 & 1.33966 \\
\hline 6.74047 & 0.00303 & 0.00015 & 1.34809 \\
\hline 6.78266 & 0.00512 & 0.00026 & 1.35653 \\
\hline 6.82437 & 0.00579 & 0.0003 & 1.36487 \\
\hline 6.86578 & 0.0046 & 0.00023 & 1.37316 \\
\hline 6.90766 & 0.00494 & 0.00025 & 1.38153 \\
\hline 6.95 & 0.00536 & 0.00027 & 1.39 \\
\hline 6.99234 & 0.00813 & 0.00041 & 1.39847 \\
\hline 7.03484 & 0.00767 & 0.00039 & 1.40697 \\
\hline 7.07766 & 0.00512 & 0.00026 & 1.41553 \\
\hline 7.12031 & 0.00606 & 0.00031 & 1.42406 \\
\hline 7.16297 & 0.00466 & 0.00024 & 1.43259 \\
\hline 7.20547 & 0.00149 & 0.00008 & 1.44109 \\
\hline 7.24828 & 0.00342 & 0.00017 & 1.44966 \\
\hline 7.29078 & 0.0058 & 0.0003 & 1.45816 \\
\hline 7.33297 & 0.00493 & 0.00025 & 1.46659 \\
\hline 7.37531 & 0.0033 & 0.00017 & 1.47506 \\
\hline 7.41766 & 0.00409 & 0.00021 & 1.48353 \\
\hline 7.45969 & 0.00715 & 0.00036 & 1.49194 \\
\hline 7.50156 & 0.0068 & 0.00035 & 1.50031 \\
\hline 7.54375 & 0.00614 & 0.00031 & 1.50875 \\
\hline 7.58625 & 0.00827 & 0.00042 & 1.51725 \\
\hline 7.62859 & 0.00831 & 0.00042 & 1.52572 \\
\hline 7.67078 & 0.00637 & 0.00032 & 1.53416 \\
\hline 7.71281 & 0.00483 & 0.00025 & 1.54256 \\
\hline 7.75484 & 0.00529 & 0.00027 & 1.55097 \\
\hline 7.79703 & 0.00727 & 0.00037 & 1.55941 \\
\hline 7.83969 & 0.00787 & 0.0004 & 1.56794 \\
\hline 7.88266 & 0.00533 & 0.00027 & 1.57653 \\
\hline 7.92531 & 0.00397 & 0.0002 & 1.58506 \\
\hline 7.9675 & 0.00396 & 0.0002 & 1.5935 \\
\hline 8.00984 & 0.00449 & 0.00023 & 1.60197 \\
\hline 8.05203 & 0.00466 & 0.00024 & 1.61041 \\
\hline 8.09375 & 0.00354 & 0.00018 & 1.61875 \\
\hline
\end{tabular}

\begin{tabular}{|c|c|c|c|}
\hline $\begin{array}{l}\text { Extension } \\
\text { [mm] }\end{array}$ & $\begin{array}{c}\text { Load } \\
\text { (N) }\end{array}$ & $\begin{array}{c}\text { Compressive stress } \\
\text { (MPa] }\end{array}$ & $\begin{array}{c}\text { Compressive strain } \\
{[\mathrm{mm} / \mathrm{mm}]}\end{array}$ \\
\hline 8.13516 & 0.00422 & 0.00021 & 1.62703 \\
\hline 8.17719 & 0.00507 & 0.00026 & 1.63544 \\
\hline 8.21953 & 0.00485 & 0.00025 & 1.64391 \\
\hline 8.26203 & 0.00716 & 0.00036 & 1.65241 \\
\hline 8.30469 & 0.00748 & 0.00038 & 1.66094 \\
\hline 8.3475 & 0.00561 & 0.00029 & 1.6695 \\
\hline 8.39 & 0.00359 & 0.00018 & 1.678 \\
\hline 8.43234 & 0.0039 & 0.0002 & 1.68647 \\
\hline 8.47453 & 0.00443 & 0.00023 & 1.69491 \\
\hline 8.51719 & 0.0044 & 0.00022 & 1.70344 \\
\hline 8.55953 & 0.00422 & 0.00021 & 1.71191 \\
\hline 8.60203 & 0.0048 & 0.00024 & 1.72041 \\
\hline 8.64453 & 0.00305 & 0.00016 & 1.72891 \\
\hline 8.68656 & 0.00161 & 0.00008 & 1.73731 \\
\hline 8.72828 & 0.0025 & 0.00013 & 1.74566 \\
\hline 8.77031 & 0.00398 & 0.0002 & 1.75406 \\
\hline 8.8125 & 0.00399 & 0.0002 & 1.7625 \\
\hline 8.85484 & 0.00495 & 0.00025 & 1.77097 \\
\hline 8.89688 & 0.00669 & 0.00034 & 1.77938 \\
\hline 8.93922 & 0.00654 & 0.00033 & 1.78784 \\
\hline 8.98203 & 0.0073 & 0.00037 & 1.79641 \\
\hline 9.025 & 0.0079 & 0.0004 & 1.805 \\
\hline 9.0675 & 0.00709 & 0.00036 & 1.8135 \\
\hline 9.11016 & 0.00298 & 0.00015 & 1.82203 \\
\hline 9.15281 & 0.00281 & 0.00014 & 1.83056 \\
\hline 9.19547 & 0.0034 & 0.00017 & 1.83909 \\
\hline 9.23781 & 0.00243 & 0.00012 & 1.84756 \\
\hline 9.27984 & 0.00328 & 0.00017 & 1.85597 \\
\hline 9.32156 & 0.00545 & 0.00028 & 1.86431 \\
\hline 9.36297 & 0.00327 & 0.00017 & 1.87259 \\
\hline 9.40484 & 0.00383 & 0.0002 & 1.88097 \\
\hline 9.44719 & 0.00802 & 0.00041 & 1.88944 \\
\hline 9.48953 & 0.0092 & 0.00047 & 1.89791 \\
\hline 9.53219 & 0.00891 & 0.00045 & 1.90644 \\
\hline 9.575 & 0.00854 & 0.00043 & 1.915 \\
\hline 9.61781 & 0.00797 & 0.00041 & 1.92356 \\
\hline 9.66016 & 0.00559 & 0.00028 & 1.93203 \\
\hline 9.70266 & 0.00547 & 0.00028 & 1.94053 \\
\hline 9.74547 & 0.00796 & 0.00041 & 1.94909 \\
\hline 9.78828 & 0.00671 & 0.00034 & 1.95766 \\
\hline 9.83078 & 0.0055 & 0.00028 & 1.96616 \\
\hline 9.87297 & 0.00586 & 0.0003 & 1.97459 \\
\hline 9.91484 & 0.00608 & 0.00031 & 1.98297 \\
\hline 9.95656 & 0.00611 & 0.00031 & 1.99131 \\
\hline 9.99828 & 0.00493 & 0.00025 & 1.99966 \\
\hline 10.04016 & 0.00649 & 0.00033 & 2.00803 \\
\hline 10.08266 & 0.00595 & 0.0003 & 2.01653 \\
\hline 10.12516 & 0.0057 & 0.00029 & 2.02503 \\
\hline 10.16766 & 0.04644 & 0.00237 & 2.03353 \\
\hline 10.21016 & 0.18831 & 0.00959 & 2.04203 \\
\hline 10.25281 & 0.56149 & 0.0286 & 2.05056 \\
\hline 10.29531 & 1.27573 & 0.06497 & 2.05906 \\
\hline 10.33813 & 2.36879 & 0.12064 & 2.06763 \\
\hline 10.38094 & 3.80982 & 0.19403 & 2.07619 \\
\hline 10.42344 & 5.5096 & 0.2806 & 2.08469 \\
\hline 10.46578 & 7.13662 & 0.36346 & 2.09316 \\
\hline 10.50813 & 8.331 & 0.42429 & 2.10162 \\
\hline 10.54984 & 9.1208 & 0.46452 & 2.10997 \\
\hline 10.59141 & 9.64498 & 0.49121 & 2.11828 \\
\hline 10.63281 & 10.35119 & 0.52718 & 2.12656 \\
\hline 10.675 & 11.46769 & 0.58404 & 2.135 \\
\hline 10.71734 & 12.94135 & 0.6591 & 2.14347 \\
\hline 10.75953 & 14.74544 & 0.75098 & 2.15191 \\
\hline 10.80188 & 16.8089 & 0.85607 & 2.16038 \\
\hline
\end{tabular}




\begin{tabular}{|c|c|c|c|}
\hline $\begin{array}{l}\text { Extension } \\
\text { (mm) }\end{array}$ & $\begin{array}{l}\text { Load } \\
\text { [N] }\end{array}$ & $\begin{array}{c}\text { Compressive stress } \\
\text { (MPa) }\end{array}$ & $\begin{array}{c}\text { Compressive strain } \\
{[\mathrm{mm} / \mathrm{mm}]}\end{array}$ \\
\hline 10.84484 & 19.02025 & 0.96869 & 2.16897 \\
\hline 10.8875 & 21.30664 & 1.08514 & 2.1775 \\
\hline 10.92969 & 23.63795 & 1.20387 & 2.18594 \\
\hline 10.97234 & 25.9896 & 1.32364 & 2.19447 \\
\hline 11.01516 & 28.39837 & 1.44632 & 2.20303 \\
\hline 11.05766 & 30.88005 & 1.57271 & 2.21153 \\
\hline 11.09953 & 33.44511 & 1.70335 & 2.21991 \\
\hline 11.14156 & 36.11368 & 1.83925 & 2.22831 \\
\hline 11.18359 & 38.89737 & 1.98103 & 2.23672 \\
\hline 11.22547 & 41.79602 & 2.12865 & 2.24509 \\
\hline 11.26734 & 44.80234 & 2.28176 & 2.25347 \\
\hline 11.30953 & 47.9259 & 2.44085 & 2.26191 \\
\hline 11.35203 & 51.21958 & 2.60859 & 2.27041 \\
\hline 11.39406 & 54.70134 & 2.78592 & 2.27881 \\
\hline 11.43656 & 58.36162 & 2.97233 & 2.28731 \\
\hline 11.47938 & 62.21656 & 3.16866 & 2.29588 \\
\hline 11.52203 & 66.28561 & 3.3759 & 2.30441 \\
\hline 11.56437 & 70.54805 & 3.59298 & 2.31288 \\
\hline 11.60687 & 74.98784 & 3.8191 & 2.32137 \\
\hline 11.64953 & 79.62549 & 4.05529 & 2.32991 \\
\hline 11.69203 & 84.46833 & 4.30194 & 2.33841 \\
\hline 11.73438 & 89.50186 & 4.55829 & 2.34687 \\
\hline 11.77672 & 94.72953 & 4.82454 & 2.35534 \\
\hline 11.81859 & 100.14928 & 5.10056 & 2.36372 \\
\hline 11.86 & 105.76311 & 5.38647 & 2.372 \\
\hline 11.90156 & 111.5918 & 5.68332 & 2.38031 \\
\hline 11.94391 & 117.7119 & 5.99502 & 2.38878 \\
\hline 11.98609 & 124.14478 & 6.32264 & 2.39722 \\
\hline 12.02813 & 130.87589 & 6.66545 & 2.40563 \\
\hline 12.07094 & 137.91554 & 7.02398 & 2.41419 \\
\hline 12.11391 & 145.31133 & 7.40064 & 2.42278 \\
\hline 12.15656 & 153.12538 & 7.79861 & 2.43131 \\
\hline 12.19875 & 161.29836 & 8.21486 & 2.43975 \\
\hline 12.24125 & 169.79974 & 8.64783 & 2.44825 \\
\hline 12.28406 & 178.78223 & 9.1053 & 2.45681 \\
\hline 12.32625 & 188.31354 & 9.59073 & 2.46525 \\
\hline 12.36828 & 198.35661 & 10.10222 & 2.47366 \\
\hline 12.41047 & 208.91579 & 10.63999 & 2.48209 \\
\hline 12.45203 & 220.08673 & 11.20893 & 2.49041 \\
\hline 12.49328 & 231.9142 & 11.81129 & 2.49866 \\
\hline 12.535 & 244.40761 & 12.44758 & 2.507 \\
\hline 12.57719 & 257.74649 & 13.12692 & 2.51544 \\
\hline 12.61922 & 272.10654 & 13.85827 & 2.52384 \\
\hline 12.66125 & 287.43784 & 14.63909 & 2.53225 \\
\hline 12.70359 & 303.74167 & 15.46944 & 2.54072 \\
\hline 12.74594 & 321.15527 & 16.3563 & 2.54919 \\
\hline 12.78797 & 339.82367 & 17.30708 & 2.55759 \\
\hline 12.83031 & 359.83475 & 18.32623 & 2.56606 \\
\hline 12.87297 & 381.34155 & 19.42157 & 2.57459 \\
\hline 12.91563 & 404.52728 & 20.60241 & 2.58313 \\
\hline 12.95781 & 429.36453 & 21.86736 & 2.59156 \\
\hline 12.99109 & 450.37894 & 22.93761 & 2.59822 \\
\hline
\end{tabular}




\section{APPENDIX E: STEP-BY-STEP PROTOCOL}

\section{Scaffold Fabrication}

1. Ultra Epsom medium grain premium salt was milled in a 550mL Eisco Labs porcelain mortar and pestle

2. Sifted salt through American Educational screen sieves set and collected the salt particles between mesh sizes 60 and 230 in a plastic tupperware

3. Milled Resomer ester-terminated 85 lactide: 15 glycolide PLGA with a molecular weight of $190,000-240,000 \mathrm{~g} / \mathrm{mol}$ in a $550 \mathrm{~mL}$ Eisco Labs porcelain mortar and pestle

4. Sifted PLGA through American Educational screen sieves set and collected the salt particles between mesh sizes 60 and 230 in a separate plastic tupperware

5. Used a scale to measure out $0.8 \mathrm{~g}$ of salt and $0.2 \mathrm{~g}$ of PLGA and vortexed the powders together in a $10 \mathrm{~mL}$ conical

6. Carefully poured the gram of salt and PLGA powder into a 5mm Type KB Precision die

7. Pressed the salt and PLGA firmly within the die using a $1 \mathrm{kN}$ press

8. Removed the pressed pellet from the die and placed it on the gas foaming chamber scaffold shelf. Six pressed pellets went on the shelf at a time

9. Sealed the gas foaming chamber and connected the quick-disconnect tubing and the pressure transducer

10. Making sure the release valve was closed, the 800psi carbon dioxide tank was opened, flooding the gas foaming chamber with carbon dioxide gas

11. Waited 22 hours

12. Connected the National Instruments USB-6002 Multifunction I/O device (DAQ) to a laptop with the National Instruments LabView program created for this project

13. Within the LabView program, set the output voltage range from $2 \mathrm{~V}$ to $2 \mathrm{~V}$, filled in the name of the person running the experiment along with the date, selected a .csv file to export to, and set the current output voltage to $0 \mathrm{~V}$

14. Connected the Electronic Positioning System on the Valworx Electric Actuated Stainless Ball Valve to a wall outlet

15. Set the current output voltage to $1.5 \mathrm{~V}$

16. Ran the LabView program

17. Disconnected the gas foaming chamber and removed the gas foamed scaffolds from the gas foaming chamber

18. Let the scaffolds soak in deionized water for 24 hours, leaving behind a porous PLGA scaffold 


\section{SEM Images of Scaffolds}

1. Scaffolds for imaging were placed in a Denton Vacuum Desk IV

2. The pressure in the Denton Vacuum Desk IV was lowered to $100 \mathrm{mTorr}$ and the scaffolds were gold sputtered for $60 \mathrm{~s}$

3. The pressure in the Denton Vacuum Desk IV was returned to ambient conditions

4. One scaffold was placed onto a locking mechanism in a JEOL JSM-6390 Scanning Electron Microscope (SEM) and the door to the SEM was shut

5. The pressure inside the SEM was lowered to 50 $\mu$ Torr

6. The high-voltage cathode in the SEM was turned on, producing an image in the JEOL JSM-6390 software

7. Four pictures were taken of each scaffold: one at 30x magnification centered on the scaffold, one at 100x magnification centered on the scaffold, one at 100x magnification directly to the 100x magnification centered image, and one at 100x magnification directly to the right of the 100x magnification centered image

8. Scalar measurements of pore sizes over $30 \mu \mathrm{m}$ were taken of the $100 \mathrm{x}$ magnification photos inside the software

9. The 100x magnification images were filtered out using the color threshold settings in Figure 3.7 in Fiji ImageJ. This filter was selected and the number of pixels filtered out was measured

10. An ANOVA was run between the number of filtered pixels between the two scaffold fabrication methods using JMP 12

\section{Cell Culture}

1. The biological safety cabinet cell culture was to be performed in was thoroughly cleaned following aseptic procedures

2. $15 \mathrm{~mL}$ of Cell Applications, Inc. human osteoblast growth medium were pipetted into a T75 flask

3. $1 \mathrm{~mL}$ of Cell Applications, Inc. adult human osteoblasts were thawed until only a small pellet of ice remained in a water bath kept at $37^{\circ} \mathrm{C}$ and then pipetted into the T-75 flask

4. The flask of osteoblasts was rocked back and forth gently to evenly distribute the osteoblasts on the bottom surface of the flask and placed in a humidified incubator maintained at $37^{\circ} \mathrm{C}$ with $5 \%$ carbon dioxide

5. Every other day, the growth medium was aspirated out, the flask washed with $5 \mathrm{~mL}$ of Dulbecco's Phosphate-Buffered Saline (dPBS), and $15 \mathrm{~mL}$ of growth media were pipetted back into the flask until $60 \%$ confluency was reached

6. Once $60 \%$ confluency was reached, the growth medium was aspirated out, the flask washed with $15 \mathrm{~mL}$ of Dulbecco's Phosphate-Buffered Saline (dPBS), and $30 \mathrm{~mL}$ of growth media were pipetted back into the flask until $80 \%$ confluency was reached 
7. Once $80 \%$ confluency was reached, the T-75 flask was ready to be expanded

8. For each flask being passed, $2 \mathrm{~mL}$ of trypsin, $5 \mathrm{~mL}$ of trypsin inhibitor, $15 \mathrm{~mL}$ of dPBS, and $8 \mathrm{~mL}$ of human osteoblast growth medium were placed in the water bath for 15 minutes then sprayed with $70 \%$ IPA and brought inside the biological safety cabinet

9. Twenty-five $\mathrm{mL}$ of growth medium for each flask being passed to was also placed in the water bath for 15 minutes then sprayed with 70\% IPA and brought inside the biological safety cabinet

10. The necessary number of new flasks were placed inside the biological safety cabinet

11. A flask containing osteoblasts had the growth medium aspirated out and was washed in $15 \mathrm{~mL}$ of dPBS

12. Two $\mathrm{mL}$ of trypsin were pipetted into the flask containing osteoblasts and the flask was rocked back and forth until it was deemed that the osteoblasts had sufficiently separated from the bottom of the flask

13. Five $\mathrm{mL}$ of trypsin inhibitor and enough human osteoblast growth medium were added to the flask such that $5 \mathrm{~mL}$ of the total contents of the flask could be passed to each new flask necessary

14. Another $25 \mathrm{~mL}$ of human osteoblast growth medium were then added to each new flask, and the new flasks were placed into the incubator

15. Growth medium was changed every day until $80 \%$ confluency was reached

16. Passage ratios were followed according to Figure 3.11

\section{Cell Freezing}

1. Each time a flask needed to frozen down, a cryovial per flask was obtained and labeled

2. A flask containing osteoblasts had the growth medium aspirated out and was washed in $15 \mathrm{~mL}$ of dPBS

3. Two $\mathrm{mL}$ of trypsin were pipetted into the flask containing osteoblasts and the flask was rocked back and forth until it was deemed that the osteoblasts had sufficiently separated from the bottom of the flask

4. Five $\mathrm{mL}$ of trypsin inhibitor and $8 \mathrm{~mL}$ of growth medium were added to the flask

5. The cell suspension was placed in a $50 \mathrm{~mL}$ conical and placed in acentrifuge opposite another $50 \mathrm{~mL}$ conical with $15 \mathrm{~mL}$ of water

6. The conical was centrifuged in an International Equipment Company model CL CLINICAL centrifuge for 4 minutes at 2,000rpm

7. One $\mathrm{mL}$ of freezing medium containing $80 \%$ human osteoblast growth medium, $10 \%$ dimethyl sulfoxide (DMSO), and 10\% fetal bovine serum (FBS) was made per cryovial plus one mL extra

8. The supernatant in the centrifuged conical was aspirated out

9. The pellet of osteoblasts was resuspended in $1 \mathrm{~mL}$ of freezing medium and mixed gently inside the pipette 
10. The freezing medium with osteoblasts was pipetted into a cryovial

11. The cryovials were placed inside a ThermoScientific Mr. Frosty freezing container and left in a $-80^{\circ} \mathrm{C}$ freezer for 24 hours before being transferred to a liquid nitrogen Dewar for long term storage

\section{Cell Counting}

1. Each time cells per volume had to be counted, a flask containing osteoblasts had the growth medium aspirated out, was washed in $15 \mathrm{~mL}$ of $\mathrm{dPBS}$, and had $2 \mathrm{~mL}$ of trypsin added to it which was gently rocked back and forth until the osteoblasts had sufficiently separated from the flask

2. Five $\mathrm{mL}$ of trypsin neutralizing solution was added to the flask

3. A micropipette was used to collect $100 \mu \mathrm{L}$ of the cell suspension which was added to a $2 \mathrm{~mL}$ collection tube

4. Outside of the biological safety cabinet, $100 \mu \mathrm{L}$ of Trypan Blue was pipetted into the same collection tube and mixed within the micropipette tip

5. The $200 \mu \mathrm{L}$ of Trypan Blue-stained osteoblast suspension was pipetted into a BRAND hemocytometer

6. The hemocytometer was placed under an optical microscope and live (transparent) and dead (blue) cells were counted

7. Reverse calculations were performed to estimate how many viable cells were in the original flask of cells

\section{Growth of Osteoblasts on Scaffolds}

1. Once the scaffolds had all been fabricated, 8 of the 9 vials of frozen osteoblasts were plated again following the same steps as the original plating procedure

2. When it was determined the 8 flasks of osteoblasts were one day away from reaching $80 \%$ confluency, 25 scaffolds were soaked in $70 \%$ ethanol for 10 minutes and washed in dPBS. Then the scaffolds were placed in a 48 -well plate, and $1 \mathrm{~mL}$ of either Cell Applications, Inc. Human Osteoblast Differentiation Medium (ODM), or dPBS in 21 of those wells according to Figure 3.13

3. The 48 -well plate was placed in the incubator for 24 hours

4. Following the cell counting procedure, the number of osteoblasts in the flasks were estimated and the volume of ODM needed to create a ratio of one million osteoblasts per $0.2 \mathrm{~mL}$ was determined

5. The osteoblasts were centrifuged at $2,000 \mathrm{rpm}$ for 4 minutes in a $50 \mathrm{~mL}$ conical, the supernatant was aspirated out, and the necessary amount of ODM was pipetted into the conical

6. The 48-well plate was removed from the incubator, the ODM and dPBS in the wells were aspirated out, and $0.2 \mathrm{~mL}$ of the ODM with osteoblasts were deposited onto 7 of the 
scaffolds 10 times each, being careful about creating bubbles or letting one's arm cross the well plate.

7. The 48-well plate was placed in the incubator for 2 hours

8. The 48 -well plate was removed from the incubator and $0.8 \mathrm{~mL}$ of ODM were added to the 7 osteoblast-containing wells, and $1 \mathrm{~mL}$ of either ODM or dPBS were added to the rest of the wells according to Figure 3.13

9. ODM and dPBS were changed every 48 hours for what was supposed to be 10 days (terminated at day 6 due to contamination)

\section{Compression Testing}

1. A Bluehill 3 program that ran compression tests that terminate at either $450 \mathrm{~N}$ or when the compression platens get too close together was created to export a .csv file with maximum load, Young's modulus, and the ultimate compressive yield of each scaffold, and the raw data of load over extension

2. The scaffolds were removed from the 48-well plate with tweezers and dried on a paper towel for 10 minutes

3. One scaffold at a time was placed in the center of the bottom compression platen of an Instron 3342 single-column tensile/compression testing machine and the Bluehill 3 program was set to run

4. When testing the scaffolds soaked in ODM or dPBS, the bottom platen would be with a Kimwipe

5. Once all tests were run, the exported data was saved to a flashdrive

\section{Staining}

1. On the same day of compression testing, the 9 scaffolds to be stained were washed in their wells with dPBS twice

2. The scaffolds were then left to sit in $70 \%$ ethanol for 10 minutes, and then the ethanol was aspirated out

3. Each scaffold was placed in a cryosectioning mold top down that was then filled halfway with optimum cutting temperature gel (OCT gel)

4. Using tweezers, each mold was submerged in liquid nitrogen inside a portable Dewar until the OCT gel was frozen

5. The frozen OCT gel blocks were then stored in a $-18^{\circ} \mathrm{C}$ freezer for 48 hours

6. One at a time an OCT gel block was removed from its mold and glued to a metal die with more OCT gel, which was then placed on a shelf inside a ThermoScientific Cryotome FSE cryostat

7. Once the new OCT gel was also frozen, the die was placed in the main apparatus of the cryostat and a cryostat blade was placed in its holder 
8. The cryostat was set to take $60 \mu \mathrm{m}$ sections and the blade was moved up against the surface of the OCT gel block such that the blade was parallel to the surface

9. The handle on the cryostat was spun to start taking sections of the OCT gel block

10. Quality sections of the OCT gel block (as in full slices containing the scaffold) were added to a VECTABOND-treated microscope slide by moving the surface of the slide up to the section on the blade and allowing the cryosection to readily adhere to the slide

11. Every once in a while, the blade had to be cleaned with 70\% IPA and dried to allow quality sections to be taken again

12. Once 8 slides (intended to be 9 slides but one OCT gel block failed to produce quality cryosections) with 5 quality cryosections were created, a 200mL 1\% Alizarin Red S (Sigma Aldrich Model \# MFCD00013049) solution was created in a glass staining jar under a fume hood

13. The 8 slides were placed in a staining tray and submerged in the Alizarin Red S solution for 4 minutes

14. The tray was pulled out of the solution and excess Alizarin Red S was shaken off

15. The tray was then briefly dipped $(\sim 1 \mathrm{~s}) 20$ times in $200 \mathrm{~mL}$ of acetone, then 20 times in $200 \mathrm{~mL}$ of a 1:1 solution of acetone and xylene, and then 20 times in $200 \mathrm{~mL}$ of pure xylene under a fume hood

16. The tray was placed on a folded paper towel in the fume hood and the acetone and xylene were properly disposed of in their corresponding waste containers

17. Images were taken of the stained slides with an Olympus CKX41 inverted microscope with the software QCapture Pro 6 at 4x magnification

18. Five images were taken of each slide, where the images were positioned to capture the most Alizarin Red S staining present in each cryosection and saved to a USB

19. The images were filtered out using the color threshold settings in Figure 3.17 in Fiji ImageJ. This filter was selected and the number of pixels filtered out was measured

20. An ANOVA and a Tukey's post-hoc test were run between the number of filtered pixels between the ODM-soaked scaffolds with osteoblasts, the ODM-soaked scaffolds without osteoblasts, and the dPBS-soaked using JMP 12 